\title{
Nickel-Catalyzed Cross-Electrophile C(sp3)-Si Coupling of Unactivated Alkyl Bromides with Vinyl Chlorosilanes
}

Jicheng Duan, ${ }^{\dagger}$ Yuquan Wang,${ }^{\dagger}$ Liangliang Qi, Peng Guo, Xiaobo Pang, Xing-Zhong Shu*

State Key Laboratory of Applied Organic Chemistry (SKLAOC), College of Chemistry and Chemical Engineering, Lanzhou University, 222 South Tianshui Road, Lanzhou, 730000, China.

\section{Table of Contents}

1. General Information........................................S2

2. Synthesis of Substrates........................................S3

3. Ni-Catalyzed Cross-Electrophile Coupling of alkyl bromides with

Vinyl Chlorosilanes.................................................S6

4. Synthetic Applications........................................S24

5. Mechanistic Investigation...................................S26

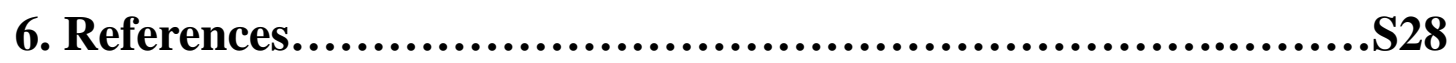

7. Copies of NMR Spectra........................................S29 


\section{General Information}

\section{Reagents and solvents}

Unless otherwise specified, all chemicals used in the preparation of starting materials were commercially available and used as received without further purification. The catalysts, reductants, additives, and other chemicals were purchased from Acros, Alfa Aesar, Aldrich, Ark Pharm, Strem Adamas, TCI and Energy chemicals, and were used directly without purification.

Anhydrous $N, \quad N$-dimethylformamide (DMF), Tetrahydrofuran (THF), and dichloromethane $\left(\mathrm{CH}_{2} \mathrm{Cl}_{2}\right)$ were purified using a solvent-purification system by passing through activated alumina columns or molecular sieves. Other solvents were analytically pure, and they were dried and purified according to the procedure from "Purification of Laboratory Chemicals"1

\section{Analytical methods}

${ }^{1} \mathrm{H},{ }^{13} \mathrm{C}$, and ${ }^{19} \mathrm{~F}$ NMR spectra were collected on a Bruker AVANCE III $400 \mathrm{MHz}$, JEOL JNM-ECS 400M and Agilent-NMR-inova $600 \mathrm{MHz}$ spectrometer at room temperature. ${ }^{1} \mathrm{H}$ NMR spectra were reported in parts per million (ppm) downfield of tetramethylsilane (TMS) and were referenced to the signal of $\mathrm{CHCl}_{3}(7.26 \mathrm{ppm}) .{ }^{13} \mathrm{C}$ NMR spectra were reported in ppm relative to residual $\mathrm{CHCl}_{3}$ (77.16 ppm). Coupling constants, $J$, are reported in hertz $(\mathrm{Hz}) .{ }^{29} \mathrm{Si}$ NMR spectra were calibrated using a chlorotrimethylsilane capillary standard at 30.92 (ppm) and were collected on Bruker AVANCE III $400 \mathrm{MHz}$ and JNM-ECS 400 spectrometers. Melting points were determined on a microscopic apparatus. IR spectra were collected using BrukerTENSOR 27 spectrometer and Agilent Technologies Cary 630 FTIR, and only major peaks were reported in $\mathrm{cm}^{-1}$. HRMS was performed on Bruker Apex II FT-ICR mass instrument (ESI) and Thermo Scientific Ultimate 3000-Q Exactive Focus QuadrupoleOrbitrap (APCI). GC analysis was performed on Thermo Scientific TRACE 1300. GCMS data was collected on Thermo Scientific TRACE DSQ GC-MS. Contact angle of glass surface was analyzed by Contact angle meter $\mathrm{SZ}_{-\mathrm{CAMB}}$. Thin layer chromatography was carried out using XINNUO SGF254 TLC plates. Flash chromatography was performed using XINNUO silica gel (200-300 mesh). 


\section{Synthesis of Substrates}

\subsection{Alkyl bromides}

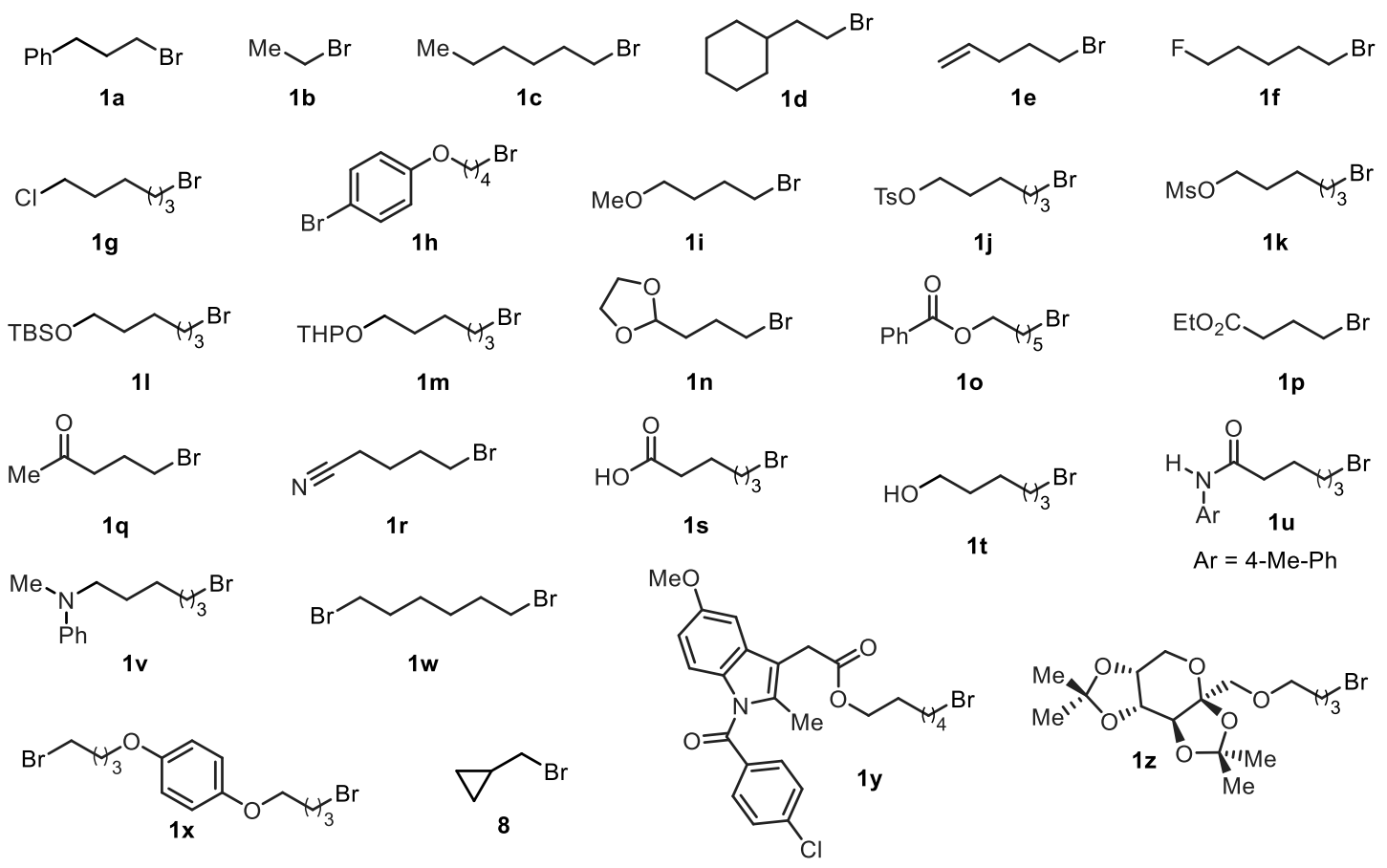

Alkyl bromides 1a-g, 1i, 1n, 1p-t, 1w, and $\mathbf{8}$ are commercially available. Alkyl bromides $1 \mathbf{h},{ }^{2} \mathbf{1 j},{ }^{3} \mathbf{1 k},{ }^{4} \mathbf{1},{ }^{5} \mathbf{1 m},{ }^{6} \mathbf{1 o},{ }^{7} 1 \mathbf{u},{ }^{8} 1 \mathbf{v},{ }^{8} 1 \mathbf{x}^{9}, 1 \mathbf{y},{ }^{10}$ and $1 \mathbf{z}^{11}$ are known compounds.

\subsection{Synthesis of vinyl chlorosilanes}

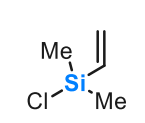

$2 a$<smiles>C=CC[Si](Cl)(Cl)C=C</smiles>

2h

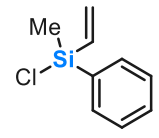

2b<smiles>C=[As](Cl)(Cl)c1ccc(F)cc1</smiles>

2c

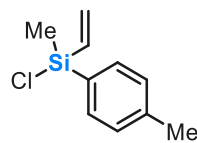

2d

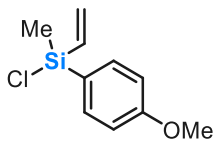

$2 \mathrm{e}$
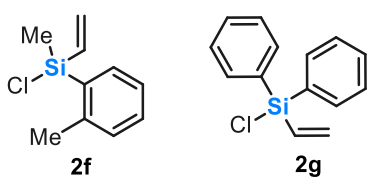
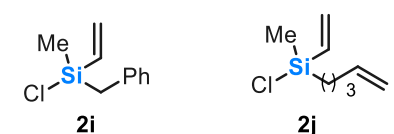

$\mathbf{2 j}$

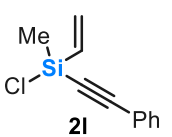

Chlorosilanes 2a-g were purchased from NY-Silicon (http://nanyuan-chem.com). Vinyl chlorosilanes $\mathbf{2} \mathbf{i},{ }^{12} \mathbf{2} \mathbf{k},{ }^{12} \mathbf{2} \mathbf{l},{ }^{12}$ and $\mathbf{2} \mathbf{m}^{12}$ are known compounds. The synthesis of new compounds and their characterization data are provided as follows. 


\section{General procedure:}

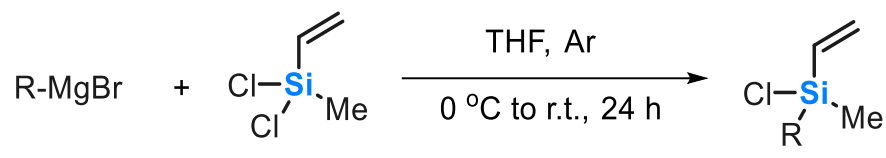

To a $50.0 \mathrm{~mL}$ dried three-necked round-bottom flask equipped with a magnetic stirring bar was added magnesium turnings $(0.48 \mathrm{~g}, 20.0 \mathrm{mmol})$, followed by a solution of alkyl bromide $(2.0 \mathrm{mmol})$ in dry THF $(5 \mathrm{~mL})$. Two grain of iodine was added and, the reaction mixture was heated to initiate the reaction. A solution of alkyl bromide $(8.0 \mathrm{mmol})$ in dry THF (15.0 mL) was added dropwise and, the reaction mixture was refluxed for $2 \mathrm{~h}$. A solution of dichloromethylvinylsilane $(1.40 \mathrm{~g}, 10.0 \mathrm{mmol})$ in THF $(10.0 \mathrm{~mL})$ was added dropwise and stirring continued for $12 \mathrm{~h}$. The reaction mixture was cooled down to room temperature and filtered. The filtrate was concentrated by rotary evaporation. The residue was distilled under vacuum to afford the desired compound.

\section{Chloro(methyl)(octyl)(vinyl)silane (2h)}<smiles>C=CC[Si](C)(Cl)CCC</smiles>

This compound was prepared from 1-bromooctane (1.90 g, 10.0 $\mathrm{mmol}$ ) and dichloro(methyl)(vinyl)silane (1.40 g, $10.0 \mathrm{mmol})$ according to General procedure A. $1.53 \mathrm{~g}, 70 \%$ yield, colorless oil.

${ }^{1}$ H NMR (400 MHz, CDCl $)$ ): $\delta$ 6.22-6.06 (m, $\left.2 \mathrm{H}\right)$, 5.92-5.86 (m, $\left.1 \mathrm{H}\right)$, 1.44-1.26 (m, $12 \mathrm{H}), 0.90-0.86(\mathrm{~m}, 5 \mathrm{H}), 0.46$ (s, $3 \mathrm{H})$.

${ }^{13}$ C NMR (100 MHz, $\left.\mathbf{C D C l}_{3}\right): \delta$ 135.4, 134.6, 33.1, 32.0, 29.3, 23.0, 22.8, 17.8, 14.2, 0.0 .

${ }^{29} \mathrm{Si}$ NMR (79 MHz, CDCl3): $\delta$ 19.4.

IR (neat, $\mathbf{c m}^{-1}$ ): 2927.8, 2854.5, 1595.1, 1465.8, 1407.1, 1255.6, 1064.7, 774.5.

Note: The HRMS of $\mathbf{2 h}$ was failed to be collected.

\section{Chloro(methyl)(pent-4-en-1-yl)(vinyl)silane (2j)}

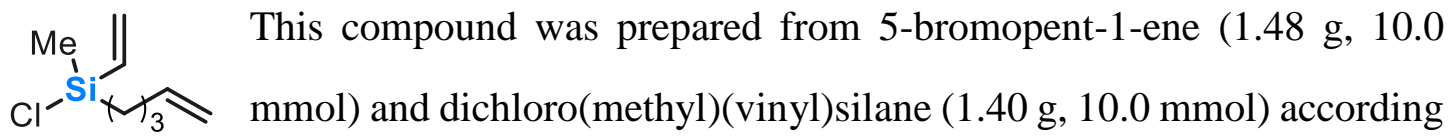
to General procedure A. $1.25 \mathrm{~g}, 72 \%$ yield, colorless oil. 
${ }^{1} \mathbf{H}$ NMR (400 MHz, CDCl $)$ ): $\delta$ 6.21-6.07 (m, $\left.2 \mathrm{H}\right)$, 5.94-5.73 (m, $\left.2 \mathrm{H}\right)$, 5.04-4.96 (m, 2 H), 2.14-2.08 (m, 2 H), 1.56-1.49 (m, 2 H), 0.92-0.87 (m, 2 H), 0.47 (s, 3H).

${ }^{13}$ C NMR (100 MHz, $\left.\mathbf{C D C l}_{3}\right): \delta 138.4,135.2,134.8,115.2,37.0,22.4,17.2,-0.03$

${ }^{29}$ Si NMR (79 MHz, CDCl3): $\delta-19.3$.

IR (neat, $\mathbf{c m}^{-1}$ ): 2925.9, 2858.4, 1641.4, 1406.1, 1255.6, 1060.8, 750.3, 526.5.

Note: The HRMS of $\mathbf{2} \mathbf{j}$ was failed to be collected. 


\section{Ni-catalyzed cross-electrophile coupling of alkyl bromides with vinyl chlorosilanes}

3.1 Optimization of reaction conditions

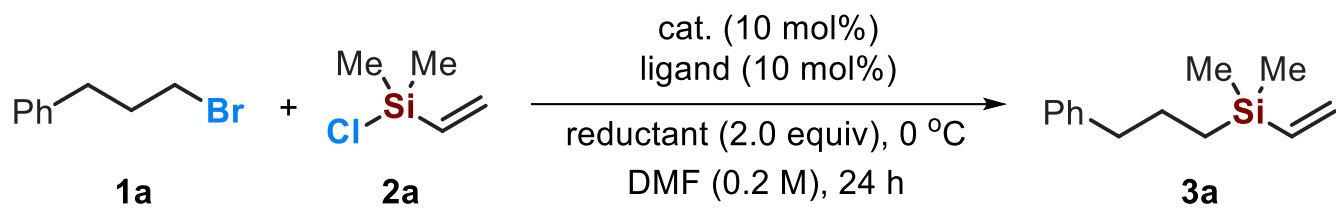

General procedure I: The procedure was conducted in an argon-filled glove box. To a reaction tube equipped with a magnetic stir bar was added catalyst $(0.01 \mathrm{mmol}, 10$ mol\%), ligand ( $0.01 \mathrm{mmol}, 10 \mathrm{~mol} \%)$, and reductant ( $0.2 \mathrm{mmol}, 2.0$ equiv). A solution of alkyl bromide $\mathbf{1 a}(19.8 \mathrm{mg}, 0.1 \mathrm{mmol})$ and chlorosilane $\mathbf{2 a}(36.0 \mathrm{mg}, 0.3 \mathrm{mmol})$ in DMF $(0.5 \mathrm{~mL})$ was added. The reaction tube was sealed and removed from the glove box. The reaction mixture was stirred at $0{ }^{\circ} \mathrm{C}$ for $24 \mathrm{~h}$. The reaction mixture was diluted with ethyl acetate $(10.0 \mathrm{~mL})$, washed with water $(5.0 \mathrm{~mL})$, brine $(5.0 \mathrm{~mL})$, and dried over anhydrous $\mathrm{Na}_{2} \mathrm{SO}_{4}$. A $0.4 \mathrm{~mL}$ of solution was collected and diluted with ethyl acetate $(2 \mathrm{~mL})$, and it was analyzed by GC. The yield was determined versus the internal standard (dodecane).

\subsection{Ni-catalyzed cross-electrophile C-Si coupling of alkyl bromides with vinyl} chlorosilanes

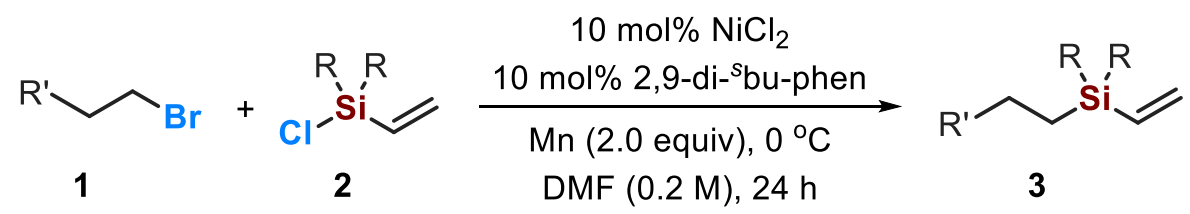

General procedure II: The procedure was conducted in an argon-filled glove box. To a reaction tube equipped with a magnetic stir bar was added $\mathrm{NiCl}_{2}(2.6 \mathrm{mg}, 0.02 \mathrm{mmol})$, 2,9-di- ${ }^{-}$bu-phen $(5.8 \mathrm{mg}, 0.02 \mathrm{mmol})$, and $\mathrm{Mn}(22.0 \mathrm{mg}, 0.4 \mathrm{mmol})$. A solution of alkyl bromide $1(0.2 \mathrm{mmol})$ and chlorosilane $2(0.2 \mathrm{mmol})$ in DMF $(1.0 \mathrm{~mL})$ was then added. The reaction tube was sealed and removed from the glove box. The reaction mixture 
was stirred at $0{ }^{\circ} \mathrm{C}$ for $24 \mathrm{~h}$. The reaction was quenched with water $(20.0 \mathrm{~mL})$, and the mixture solution was extracted with ethyl acetate $(3 \times 15.0 \mathrm{~mL})$. The combined organic layers were washed with water, brine, dried over anhydrous $\mathrm{Na}_{2} \mathrm{SO}_{4}$, and concentrated under reduced pressure. The residue was purified by flash chromatography on silica gel to afford product 3 .

\subsection{Characterization Data of new compounds}

\section{Methyl(phenyl)(3-phenylpropyl)(vinyl)silane (3a)}

Me Me This compound was prepared according to General procedure II from the reaction of $\mathbf{1 a}(39.8 \mathrm{mg}, 0.2 \mathrm{mmol})$ and $\mathbf{2 a}(72.4 \mathrm{mg}, 0.6$ mmol). $37.2 \mathrm{mg}$, 91\% yield, colorless oil. $\mathrm{R}_{\mathrm{f}}=0.6$ (silica gel, petroleum ether).

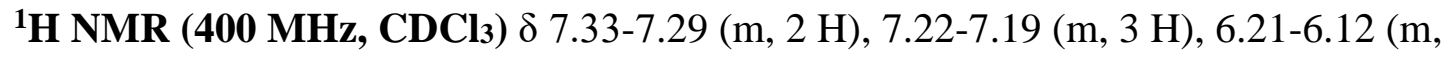
$1 \mathrm{H}), 6.00-5.95(\mathrm{~m}, 1 \mathrm{H}), 5.72-5.66(\mathrm{~m}, 1 \mathrm{H}), 2.66(\mathrm{t}, J=8.0 \mathrm{~Hz}, 2 \mathrm{H}), 1.70-1.62(\mathrm{~m}, 2$ H), 0.67-0.63 (m, $2 \mathrm{H}), 0.09$ (s, $6 \mathrm{H})$.

${ }^{13}$ C NMR (100 MHz, CDCl3) $\delta 142.8,139.2,131.7,128.6,128.4,125.8,40.0,26.2$, $15.4,-3.3$

${ }^{29} \mathrm{Si}$ NMR (79 MHz, $\left.\mathrm{CDCl}_{3}\right): \delta-5.2$.

IR (neat, cm$^{-1}$ ): 3027, 2956, 2926, 1734, 1248, 1009, 833, 744, 698.

HRMS (APCI): $[\mathrm{M}+\mathrm{H}]^{+}$calcd for $\mathrm{C}_{13} \mathrm{H}_{21} \mathrm{Si} 205.1407$, found: 205.1393.

\section{Ethyl(methyl)(phenyl)(vinyl)silane (3b)}

This compound was prepared according to General procedure II from Me $25.7 \mathrm{mg}, 73 \%$ yield, colorless oil. $\mathrm{R}_{\mathrm{f}}=0.6$ (silica gel, petroleum ether).

${ }^{1}$ H NMR (400 MHz, CDCl$)$ ) $\delta$ 7.55-7.54 (m, 2 H), 7.39-7.37 (m, 3 H), 6.36-6.27 (m, $1 \mathrm{H}), 6.14-6.09$ (m, $1 \mathrm{H}), 5.82-5.76(\mathrm{~m}, 1 \mathrm{H}), 1.01$ (t, $J=7.6 \mathrm{~Hz}, 3 \mathrm{H}), 0.90-0.86$ (m, 2 $\mathrm{H}), 0.36(\mathrm{~s}, 3 \mathrm{H})$.

${ }^{13}$ C NMR (100 MHz, CDCl3) $\delta 137.6,136.8,134.3,133.5,129.1,127.9,7.5,6.0$, 5.4 .

${ }^{29} \mathrm{Si}$ NMR (79 MHz, CDCl 3$): ~ \delta 0.5$. 
IR (neat, $\mathbf{c m}^{-1}$ ): 2958, 2922, 1651, 1403, 1113, 1010, 792, 699.

HRMS (APCI): $[\mathrm{M}+\mathrm{H}]^{+}$calcd for $\mathrm{C}_{11} \mathrm{H}_{17} \mathrm{Si}$ 177.1094, found: 177.1088.

Hexyl(methyl)(phenyl)(vinyl)silane (3c)

$\mathrm{Ph} \mathrm{Me}$ This compound was prepared according to General procedure Me II from the reaction of $\mathbf{1 c}(33.0 \mathrm{mg}, 0.2 \mathrm{mmol})$ and $\mathbf{2 b}(110.0$ $\mathrm{mg}, 0.6 \mathrm{mmol}$ ). $39.9 \mathrm{mg}, 86 \%$ yield, colorless oil. $\mathrm{R}_{\mathrm{f}}=0.6$ (silica gel, petroleum ether). ${ }^{1}$ H NMR (400 MHz, CDCl 3$) \delta$ 7.54-7.52 (m, 2 H), 7.38-7.36 (m, 3 H), 6.35-6.26 (m, $1 \mathrm{H}), 6.12-6.07(\mathrm{~m}, 1 \mathrm{H})$, 5.80-5.74 (m, $1 \mathrm{H}), 1.37-1.28$ (m, $8 \mathrm{H}), 0.90-0.84(\mathrm{~m}, 5 \mathrm{H})$, $0.35(\mathrm{~s}, 3 \mathrm{H})$.

${ }^{13}$ C NMR (100 MHz, CDCl3) $\delta 137.9,137.2,134.2,133.4,129.1,127.9,33.4,31.7$, 23.9, 22.8, 14.3, 14.2, -4.9.

${ }^{29} \mathrm{Si}$ NMR (79 MHz, CDCl 3$): \delta-9.6$.

IR (neat, $\mathbf{c m}^{-1}$ ): 3052, 2960, 2924, 2365, 1429, 1251, 1113, 952, 792.

HRMS (APCI): $[\mathrm{M}+\mathrm{H}]^{+}$calcd for $\mathrm{C}_{15} \mathrm{H}_{25} \mathrm{Si} 233.1720$, found: 233.1740 .

\section{(2-Cyclohexylethyl)(methyl)(phenyl)(vinyl)silane (3d)}

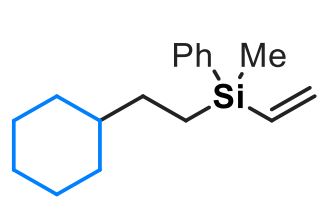

This compound was prepared according to General procedure II from the reaction of $\mathbf{1 d}(38.2 \mathrm{mg}, 0.2 \mathrm{mmol})$ and $\mathbf{2 b}(110.0 \mathrm{mg}$, $0.6 \mathrm{mmol}$ ). $45.9 \mathrm{mg}, 89 \%$ yield, colorless oil. $\mathrm{R}_{\mathrm{f}}=0.6$ (silica gel, petroleum ether).

${ }^{1}$ H NMR (400 MHz, CDCl $)$ ) $\delta$ 7.56-7.53 (m, 2 H), 7.39-7.37 (m, 3 H), 6.36-6.27 (m, $1 \mathrm{H}), 6.13-6.08(\mathrm{~m}, 1 \mathrm{H})$, 5.81-5.75 (m, $1 \mathrm{H}), 1.78-1.65(\mathrm{~m}, 5 \mathrm{H}), 1.30-1.13(\mathrm{~m}, 6 \mathrm{H})$, 0.90-0.85 (m, $4 \mathrm{H}), 0.36$ (s, $3 \mathrm{H})$.

${ }^{13}$ C NMR (100 MHz, CDCl 3$) \delta 137.9,137.2,134.2,133.4,129.1,127.9,40.9,33.1$, $31.3,26.9,26.6,11.1,-4.9$.

${ }^{29} \mathrm{Si}$ NMR (79 MHz, $\left.\mathrm{CDCl}_{3}\right): \delta-9.2$.

IR (neat, cm$^{-1}$ ): 3050, 2922, 2853, 1646, 1448, 1251, 1113, 734, 699.

HRMS (APCI): $[\mathrm{M}+\mathrm{H}]{ }^{+}$calcd for $\mathrm{C}_{17} \mathrm{H}_{27} \mathrm{Si} 259.1877$, found: 259.1895 . 


\section{Methyl(pent-4-en-1-yl)(phenyl)(vinyl)silane (3e)}

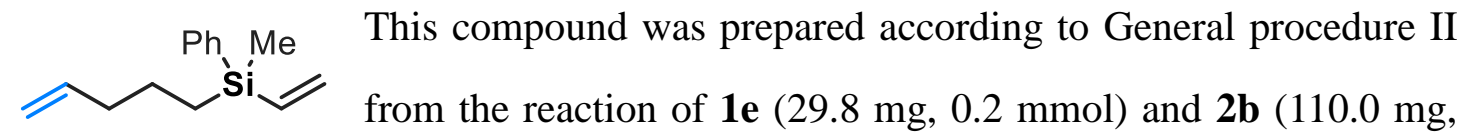

$0.6 \mathrm{mmol}$ ). $29.8 \mathrm{mg}, 69 \%$ yield, colorless oil. $\mathrm{R}_{\mathrm{f}}=0.6$ (silica gel, petroleum ether).

${ }^{1}$ H NMR (400 MHz, CDCl 3$) \delta$ 7.54-7.52 (m, 2 H), 7.38-7.36 (m, 3 H), 6.35-6.26 (m, $1 \mathrm{H})$, 6.13-6.08 (m, $1 \mathrm{H})$, 5.85-5.75 (m, $2 \mathrm{H})$, 5.02-4.95 (m, $2 \mathrm{H})$, 2.13-2.07 (m, $2 \mathrm{H})$, 1.51-1.43 (m, 2 H), 0.90-0.86 (m, 2 H), 0.36 (s, 3 H).

${ }^{13}$ C NMR (100 MHz, CDCl $) \delta 138.9,137.7,137.0,134.2,133.5,129.2,127.9$, $114.8,37.7,23.4,13.8,-4.9$.

${ }^{29} \mathrm{Si}$ NMR (79 MHz, CDCl3): $\delta-9.6$.

IR (neat, cm$^{-1}$ ): 3071, 2926, 2857, 1642, 1429, 1252, 1113, 911, 788.

HRMS (APCI): $[\mathrm{M}+\mathrm{H}]{ }^{+}$calcd for $\mathrm{C}_{14} \mathrm{H}_{21} \mathrm{Si} 217.1407$, found: 217.1426.

\section{(5-Fluoropentyl)(methyl)(phenyl)(vinyl)silane (3f)}

This compound was prepared according to General procedure
II from the reaction of $\mathbf{1 f}(33.8 \mathrm{mg}, 0.2 \mathrm{mmol})$ and $\mathbf{2 b}(110.0$ $\mathrm{mg}, 0.6 \mathrm{mmol}$ ). $43.0 \mathrm{mg}, 91 \%$ yield, colorless oil. $\mathrm{R}_{\mathrm{f}}=0.6$ (silica gel, petroleum ether). ${ }^{1} \mathbf{H}$ NMR (400 MHz, CDCl $)$ ) 7.52-7.50 (m, 2 H), 7.36-7.34 (m, 3 H), 6.32-6.24 (m, $1 \mathrm{H}), 6.11-6.06(\mathrm{~m}, 1 \mathrm{H}), 5.79-5.73(\mathrm{~m}, 1 \mathrm{H}), 4.46(\mathrm{t}, J=6.4 \mathrm{~Hz}, 1 \mathrm{H}), 4.34(\mathrm{t}, J=6.4$ $\mathrm{Hz}, 1 \mathrm{H}), 1.72-1.60$ (m, $2 \mathrm{H}), 1.46-1.37$ (m, $4 \mathrm{H}), 0.88-0.84$ (m, $2 \mathrm{H}), 0.34$ (s, $3 \mathrm{H})$. ${ }^{13} \mathrm{C}$ NMR (100 MHz, $\left.\mathbf{C D C l}_{3}\right) \delta 137.6,136.9,134.2,133.6,129.2,127.9,84.3$ (d, $J_{C-F}$ $=163.0 \mathrm{~Hz}), 30.2\left(\mathrm{~d}, J_{C-F}=19.0 \mathrm{~Hz}\right), 29.1\left(\mathrm{~d}, J_{C-F}=5.0 \mathrm{~Hz}\right), 23.6,14.2,-4.9$.

${ }^{29} \mathrm{Si}$ NMR (79 MHz, CDCl3): $\delta-9.7$.

IR (neat, cm$^{-1}$ ): 2935, 2861, 2373, 1655, 1405, 1113, 1008,788, 701

HRMS (APCI): $[\mathrm{M}+\mathrm{H}]^{+}$calcd for $\mathrm{C}_{14} \mathrm{H}_{22} \mathrm{FSi} 237.1469$, found: 237.1449.

\section{(5-Chloropentyl)(methyl)(phenyl)(vinyl)silane (3g)}

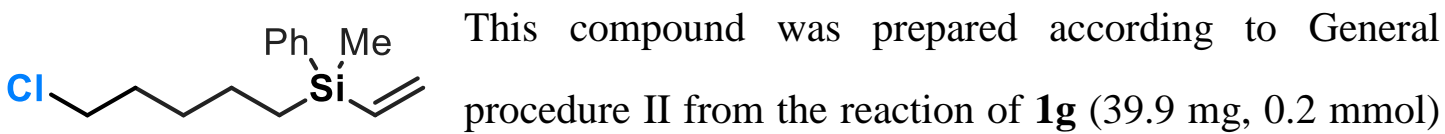
and $2 \mathbf{b}$ (110.0 mg, $0.6 \mathrm{mmol}$ ). $47.4 \mathrm{mg}, 94 \%$ yield, colorless oil. $\mathrm{R}_{\mathrm{f}}=0.6$ (silica gel, petroleum ether). 
${ }^{1} \mathbf{H}$ NMR (400 MHz, CDCl$)$ ): $\delta$ 7.52-7.49 (m, $\left.2 \mathrm{H}\right)$, 7.36-7.35 (m, $\left.3 \mathrm{H}\right)$, 6.32-6.24 (m, $1 \mathrm{H}), 6.11-6.06(\mathrm{~m}, 1 \mathrm{H}), 5.78-5.72(\mathrm{~m}, 1 \mathrm{H}), 3.50(\mathrm{t}, J=6.8 \mathrm{~Hz}, 2 \mathrm{H}), 1.79-1.72(\mathrm{~m}, 2$ H), 1.50-1.33 (m, 4 H), 0.87-0.83 (m, $2 \mathrm{H}), 0.34$ (s, $3 \mathrm{H})$.

${ }^{13}$ C NMR (100 MHz, CDCl3): $\delta$ 137.6, 136.9, 134.2, 133.6, 129.2, 127.9, 45.2, 32.4, $30.8,23.3,14.2,-4.9$.

${ }^{29} \mathrm{Si}$ NMR (79 MHz, CDCl 3$): \delta-9.7$.

IR (neat, $\mathbf{c m}^{-1}$ ): 3055, 2961, 1593, 1405, 1256, 1118, 1057, 796, 699.

HRMS (APCI): $[\mathrm{M}+\mathrm{H}]{ }^{+}$calcd for $\mathrm{C}_{14} \mathrm{H}_{22} \mathrm{ClSi} 253.1174$, found: 253.1184 .

(4-(4-Bromophenoxy)butyl)(methyl)(phenyl)(vinyl)silane (3h)

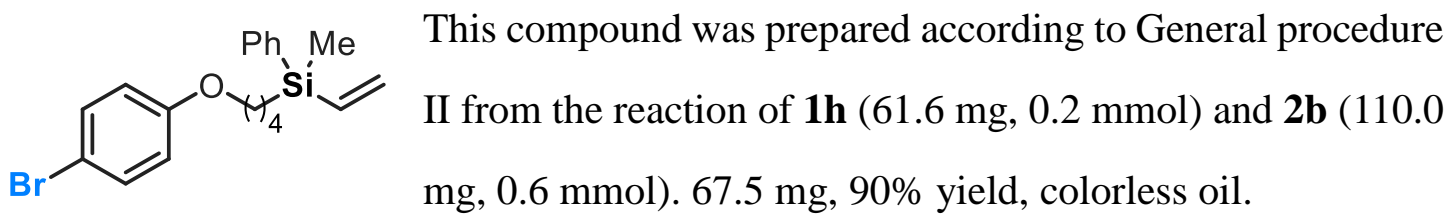

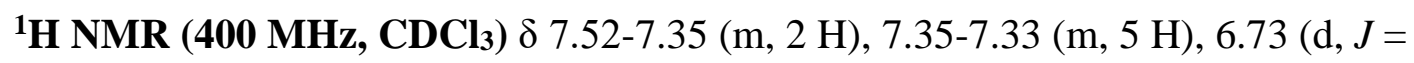
$8.8 \mathrm{~Hz}, 2 \mathrm{H}), 6.33-6.24(\mathrm{~m}, 1 \mathrm{H}), 6.11-6.06(\mathrm{~m}, 1 \mathrm{H}), 5.79-5.73(\mathrm{~m}, 1 \mathrm{H}), 3.88(\mathrm{t}, J=$ $6.4 \mathrm{~Hz}, 2 \mathrm{H}), 1.81-1.77$ (m, 2 H), 1.53-1.51 (m, $2 \mathrm{H}), 0.92-0.87$ (m, $2 \mathrm{H}), 0.35$ (s, 3 $H$ ). $R_{\mathrm{f}}=0.4$ ( silica gel, petroleum ether/ethyl acetate $=50: 1$ ).

${ }^{13}$ C NMR (100 MHz, CDCl3) $\delta$ 158.4, 137.4, 136.8, 134.2, 133.7, 132.3, 129.2, $128.0,116.5,112.7,67.9,32.9,20.4,14.0,-4.9$.

${ }^{29} \mathrm{Si}$ NMR (79 MHz, CDCl3): $\delta$-9.6.

IR (neat, $\mathbf{c m}^{-1}$ ): 3051, 2943, 2876, 1593, 1489, 1247, 1113, 794, 736.

HRMS (ESI): $\left[\mathrm{M}+\mathrm{NH}_{4}\right]^{+}$calcd for $\mathrm{C}_{19} \mathrm{H}_{27} \mathrm{BrNOSi}$ 392.1040, found: 392.1042 .

(4-Methoxybutyl)(methyl)(phenyl)(vinyl)silane (3i)

This compound was prepared according to General procedure II from the reaction of $\mathbf{1 i}(33.4 \mathrm{mg}, 0.2 \mathrm{mmol})$ and $\mathbf{2 b}(110.0$ $\mathrm{mg}, 0.6 \mathrm{mmol}$ ). $39.8 \mathrm{mg}, 85 \%$ yield, colorless oil. $\mathrm{R}_{\mathrm{f}}=0.4$ (silica gel, petroleum ether/ethyl acetate $=50: 1$ ).

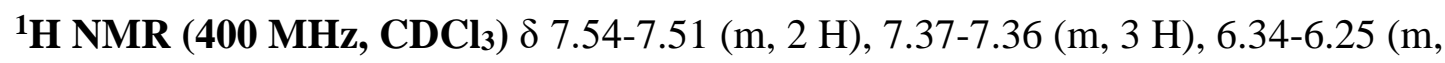
$1 \mathrm{H}), 6.12-6.07$ (m, $1 \mathrm{H}), 5.80-5.74(\mathrm{~m}, 1 \mathrm{H}), 3.36$ (t, J=6.4 Hz, $2 \mathrm{H}), 3.32(\mathrm{~s}, 3 \mathrm{H})$, 1.66-1.59 (m, 2 H), 1.47-1.39 (m, 2 H), 0.90-0.86 (m, 2 H), 0.36 (s, 3 H). 
${ }^{13}$ C NMR (100 MHz, CDCl $) \delta$ 137.6, 137.0, 134.2, 133.5, 129.1, 127.9, 72.7, 58.7, $33.6,20.6,14.2,-4.9$.

${ }^{29} \mathrm{Si}$ NMR (79 MHz, CDCl 3$): \delta-9.6$.

IR (neat, $\mathbf{c m}^{-1}$ ): 3051, 2932, 2864, 1429, 1252, 1118, 1010, 796, 701

HRMS (ESI): $[\mathrm{M}+\mathrm{H}]{ }^{+}$calcd for $\mathrm{C}_{14} \mathrm{H}_{23} \mathrm{OSi}$ 235.1513, found: 235.1514 .

\section{6-(Methyl(phenyl)(vinyl)silyl)hexyl 4-methylbenzenesulfonate (3j)}

$\mathrm{Ph}, \mathrm{Me}$ This compound was prepared according to General procedure

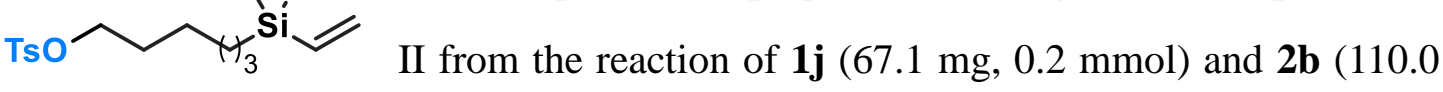
$\mathrm{mg}, 0.6 \mathrm{mmol}$ ). $53.9 \mathrm{mg}, 67 \%$ yield, colorless oil. $\mathrm{R}_{\mathrm{f}}=0.6$ (silica gel, petroleum ether/ethyl acetate $=20: 1$ )

${ }^{1}$ H NMR (400 MHz, CDCl3) $\delta 7.79$ (d, $J=8.4$ Hz, 2 H), 7.51-7.49 (m, 2 H), 7.36$7.32(\mathrm{~m}, 5 \mathrm{H}), 6.31-6.23(\mathrm{~m}, 1 \mathrm{H}), 6.10-6.06(\mathrm{~m}, 1 \mathrm{H}), 5.78-5.72(\mathrm{~m}, 1 \mathrm{H}), 4.00(\mathrm{t}, J=$ $6.8 \mathrm{~Hz}, 2 \mathrm{H}), 2.44$ (s, $3 \mathrm{H}), 1.62-1.59$ (m, $2 \mathrm{H}), 1.27$ (m, $6 \mathrm{H}), 0.82-0.78$ (m, $2 \mathrm{H})$, $0.33(\mathrm{~s}, 3 \mathrm{H})$.

${ }^{13}$ C NMR (100 MHz, CDCl3) $\delta$ 144.7, 137.6, 136.9, 134.2, 133.5, 133.4, 129.9, $129.1,128.0,127.9,70.8,32.9,28.9,25.1,23.6,21.7,14.1,-4.9$.

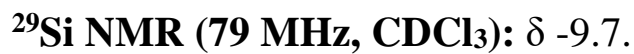

IR (neat, $\mathbf{c m}^{-1}$ ): 3051, 2928, 2857, 1599, 1362, 1178, 950, 790, 665.

HRMS (ESI): $[\mathrm{M}+\mathrm{Na}]^{+}$calcd for $\mathrm{C}_{22} \mathrm{H}_{30} \mathrm{NaO}_{3} \mathrm{SSi} 425.1577$, found: 425.1575 .

\section{6-(Methyl(phenyl)(vinyl)silyl)hexyl methanesulfonate (3k)}

$\mathrm{Ph}$ Me This compound was prepared according to General procedure

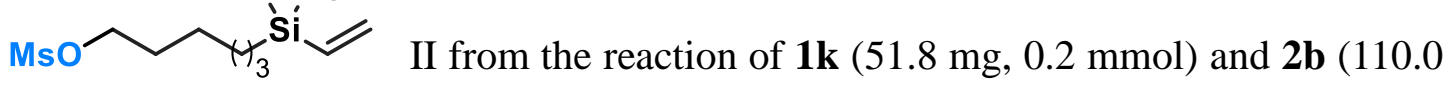
$\mathrm{mg}, 0.6 \mathrm{mmol}$ ). $48.2 \mathrm{mg}, 74 \%$ yield, colorless oil. $\mathrm{R}_{\mathrm{f}}=0.6$ (silica gel, petroleum ether/ethyl acetate $=20: 1$ ).

${ }^{1}$ H NMR (600 MHz, CDCl 3$) \delta$ 7.51-7.50 (m, 2 H), 7.36-7.35 (m, 3 H), 6.31-6.25 (m, $1 \mathrm{H}), 6.10-6.07$ (m, $1 \mathrm{H})$, 5.78-5.73 (m, $1 \mathrm{H}), 4.19$ (t, J = 6.6 Hz, $2 \mathrm{H}), 2.99$ (s, $3 \mathrm{H})$, 1.73-1.70 (m, 2 H), 1.37 (m, $6 \mathrm{H}), 0.87-0.83$ (m, $2 \mathrm{H}), 0.35$ (s, $3 \mathrm{H})$.

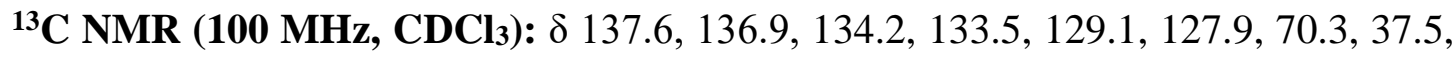
$33.0,29.1,25.2,23.7,14.1,-4.9$. 
${ }^{29} \mathrm{Si}$ NMR (79 MHz, CDCl 3$): \delta-9.7$.

IR (neat, cm-1): 2928, 2861, 1429, 1357, 1176, 949, 796, 747.

HRMS (ESI): [M+Na] ${ }^{+}$calcd for $\mathrm{C}_{16} \mathrm{H}_{26} \mathrm{NaO}_{3} \mathrm{SSi} 349.1264$, found: 349.1264 .

tert-Butyldimethyl((6-(methyl(phenyl)(vinyl)silyl)hexyl)oxy)silane (3I)

This compound was prepared according to General
procedure II from the reaction of $11(59.1 \mathrm{mg}, 0.2 \mathrm{mmol})$ and

2b (110.0 mg, $0.6 \mathrm{mmol}$ ). $45.0 \mathrm{mg}, 62 \%$ yield, colorless oil. $\mathrm{R}_{\mathrm{f}}=0.5$ (silica gel, petroleum ether/ethyl acetate $=100: 1$ ).

${ }^{1}$ H NMR (400 MHz, CDCl 3$)$ 87.52-7.50 (m, 2 H), 7.35-7.34 (m, 3 H), 6.32-6.23 (m, $1 \mathrm{H}), 6.09-6.05(\mathrm{~m}, 1 \mathrm{H}), 5.78-5.72(\mathrm{~m}, 1 \mathrm{H}), 3.58(\mathrm{t}, J=8.0 \mathrm{~Hz}, 2 \mathrm{H}), 1.51-1.44(\mathrm{~m}, 2$ H), 1.34-1.31 (m, $6 \mathrm{H}), 0.89$ (s, $9 \mathrm{H}), 0.87-0.84(\mathrm{~m}, 2 \mathrm{H}), 0.33$ (s, $3 \mathrm{H}), 0.04$ (s, $6 \mathrm{H})$. ${ }^{13}$ C NMR (100 MHz, CDCl $) \delta$ 137.9, 137.2, 134.2, 133.4, 129.1, 127.9, 63.5, 33.5, $32.9,26.1,25.6,23.9,18.5,14.2,-4.9,-5.1$.

${ }^{29} \mathrm{Si}$ NMR (79 MHz, $\left.\mathrm{CDCl}_{3}\right): \delta-18.8,-9.7$.

IR (neat, $\mathbf{c m}^{-1}$ ): 2932, 2861, 2365, 1251, 1103, 913, 837, 781, 699.

HRMS (ESI): $[\mathrm{M}+\mathrm{H}]^{+}$calcd for $\mathrm{C}_{21} \mathrm{H}_{39} \mathrm{OSi}_{2}$ 363.2534, found: 363.2533 .

Methyl(phenyl)(6-((tetrahydro-2H-pyran-2-yl)oxy)hexyl)(vinyl)silane (3m)

This compound was prepared according to General procedure $\mathrm{mg}, 0.6 \mathrm{mmol}$ ). $60.6 \mathrm{mg}, 91 \%$ yield, colorless oil. $\mathrm{R}_{\mathrm{f}}=0.6$ (silica gel, petroleum ether/ethyl acetate $=50: 1$ ).

${ }^{1}$ H NMR (400 MHz, CDCl3) $\delta$ 7.53-7.50 (m, 2 H), 7.36-7.35 (m, 3 H), 6.33-6.24 (m, $1 \mathrm{H})$, 6.10-6.06 (m, $1 \mathrm{H})$, 5.78-5.72 (m, $1 \mathrm{H}), 4.58-4.56$ (m, $1 \mathrm{H}), 3.90-3.84(\mathrm{~m}, 1 \mathrm{H})$, 3.75-3.69 (m, $1 \mathrm{H}), 3.53-3.47$ (m, $1 \mathrm{H}), 3.30-3.34(\mathrm{~m}, 1 \mathrm{H}), 1.87-1.79(\mathrm{~m}, 1 \mathrm{H}), 1.75-$ $1.69(\mathrm{~m}, 1 \mathrm{H}), 1.63-1.49(\mathrm{~m}, 6 \mathrm{H}), 1.36(\mathrm{~m}, 6 \mathrm{H}), 0.87-0.83(\mathrm{~m}, 2 \mathrm{H}), 0.34(\mathrm{~s}, 3 \mathrm{H})$. ${ }^{13}$ C NMR (100 MHz, CDCl3) $\delta 137.8,137.1,134.2,133.4,129.1,127.9,99.0,67.8$, $62.5,33.5,30.9,29.8,26.0,25.7,23.8,19.9,14.2,-4.9$.

${ }^{29} \mathrm{Si}$ NMR (79 MHz, CDCl 3$): \delta-9.6$.

IR (neat, cm-1): 3445, 3050, 2932, 2857, 1646, 1429, 1116, 1034, 701. 
HRMS (ESI): $[\mathrm{M}+\mathrm{Na}]^{+}$calcd for $\mathrm{C}_{20} \mathrm{H}_{32} \mathrm{NaO}_{2} \mathrm{Si}$ 355.2064, found: 355.2063.

\section{(3-(1,3-Dioxolan-2-yl)propyl)(methyl)(phenyl)(vinyl)silane (3n)}

This compound was prepared according to General procedure II
from the reaction of $1 \mathbf{n}(39.0 \mathrm{mg}, 0.2 \mathrm{mmol})$ and $\mathbf{2 b}(110.0 \mathrm{mg}$, $0.6 \mathrm{mmol}$ ). $28.8 \mathrm{mg}, 55 \%$ yield, colorless oil. $\mathrm{R}_{\mathrm{f}}=0.6$ (silica gel, petroleum ether/ethyl acetate $=50: 1)$.

${ }^{1}$ H NMR (400 MHz, CDCl3) $\delta$ 7.53-7.50 (m, 2 H), 7.36-7.35 (m, 3 H), 6.33-6.25 (m, $1 \mathrm{H}), 6.11-6.07(\mathrm{~m}, 1 \mathrm{H}), 5.79-5.73(\mathrm{~m}, 1 \mathrm{H}), 4.82$ (t, $J=4.8 \mathrm{~Hz}, 1 \mathrm{H}), 3.96-3.90(\mathrm{~m}, 2$ H), 3.87-3.81 (m, 2 H), 1.73-1.68 (m, 2 H), 1.54-1.46 (m, 2 H), 0.93-0.88 (m, 2 H), $0.35(\mathrm{~s}, 3 \mathrm{H})$.

${ }^{13}$ C NMR (100 MHz, CDCl3) $\delta$ 137.5, 136.8, 134.2, 133.7, 129.2, 127.9, 104.6, 64.9, $37.8,18.7,14.5,-4.9$.

${ }^{29} \mathrm{Si}$ NMR (79 MHz, CDCl 3$): \delta-9.7$.

IR (neat, $\mathbf{c m}^{-1}$ ): 2947, 2881, 1655, 1405, 1252, 1113, 1012, 798, 701.

HRMS (ESI): $[\mathrm{M}+\mathrm{Na}]^{+}$calcd for $\mathrm{C}_{15} \mathrm{H}_{22} \mathrm{NaO}_{2} \mathrm{Si} 285.1281$, found: 285.1280.

6-(Mimethyl(vinyl)silyl)hexyl benzoate (3o)

O Me Me This compound was prepared according to General procedure II $\mathrm{Ph}_{\mathrm{O}} \widehat{\mathrm{Y}}_{5}^{\mathrm{Si}}=$ from the reaction of $\mathbf{1 0}(57.0 \mathrm{mg}, 0.2 \mathrm{mmol})$ and $\mathbf{2 b}(110.0 \mathrm{mg}$, $0.6 \mathrm{mmol}$ ). $46.4 \mathrm{mg}, 80 \%$ yield, colorless oil. $\mathrm{R}_{\mathrm{f}}=0.5$ (silica gel, petroleum ether/ethyl acetate $=20: 1)$.

${ }^{1}$ H NMR (400 MHz, CDCl $)$ ) 8.06-8.04 (m, 2 H), 7.57-7.53 (m, 1 H), 7.46-7.42 (m, $2 \mathrm{H}), 6.19-6.10(\mathrm{~m}, 1 \mathrm{H}), 5.97-5.92(\mathrm{~m}, 1 \mathrm{H}), 5.70-5.64(\mathrm{~m}, 1 \mathrm{H}), 4.32(\mathrm{t}, J=6.8 \mathrm{~Hz}, 2$ H), 1.80-1.73 (m, $2 \mathrm{H}), 1.45-1.29$ (m, $6 \mathrm{H}), 0.59-0.55(\mathrm{~m}, 2 \mathrm{H}), 0.06(\mathrm{~s}, 6 \mathrm{H})$.

${ }^{13}$ C NMR (100 MHz, CDCl 3$) \delta 166.8,139.4,132.9,131.5,130.7,129.7,128.4,65.3$, $33.3,28.8,25.9,23.9,15.4,-3.2,-3.4$.

${ }^{29} \mathrm{Si}$ NMR (79 MHz, CDCl3): $\delta-9.6$.

IR (neat, $\mathbf{c m}^{-1}$ ): 3049, 2926, 2859, 2348, 1724, 1452, 1275, 1115, 710.

HRMS (ESI): $[\mathrm{M}+\mathrm{Na}]^{+}$calcd for $\mathrm{C}_{17} \mathrm{H}_{26} \mathrm{NaO}_{2} \mathrm{Si} 313.1594$, found: 313.1594 .

Ethyl 4-(methyl(phenyl)(vinyl)silyl)butanoate (3p) 
$\mathrm{Ph}$ Me This compound was prepared according to General procedure $\mathrm{EtO}_{2} \mathrm{C} \sim \mathrm{Si}$ from the reaction of $\mathbf{1 p}(39.0 \mathrm{mg}, 0.2 \mathrm{mmol})$ and $\mathbf{2 b}(110.0$ $\mathrm{mg}, 0.6 \mathrm{mmol}$ ). $46.1 \mathrm{mg}, 88 \%$ yield, colorless oil. $\mathrm{R}_{\mathrm{f}}=0.5$ (silica gel, petroleum ether/ethyl acetate $=20: 1$ ).

${ }^{1}$ H NMR (400 MHz, CDCl$)$ ) $\delta$ 7.53-7.51 (m, 2 H), 7.37-7.35 (m, 3 H), 6.34-6.25 (m, $1 \mathrm{H}), 6.13-6.08(\mathrm{~m}, 1 \mathrm{H}), 5.81-5.75(\mathrm{~m}, 1 \mathrm{H}), 4.15-4.10(\mathrm{~m}, 2 \mathrm{H}), 2.34(\mathrm{t}, J=8.0 \mathrm{~Hz}, 2$ H), 1.74-1.66 (m, $2 \mathrm{H}), 1.25$ (t, $J=8.0 \mathrm{~Hz}, 3 \mathrm{H}), 0.91-0.87(\mathrm{~m}, 2 \mathrm{H}), 0.37$ (s, $3 \mathrm{H})$. ${ }^{13}$ C NMR (100 MHz, CDCl$) ~ \delta 173.6,137.2,136.5,134.2,133.9,129.3,128.0,60.3$, $37.9,19.7,14.4,14.1,-5.0$.

${ }^{29} \mathrm{Si}$ NMR (79 MHz, CDCl3): $\delta$-9.8.

IR (neat, $\mathbf{c m}^{-1}$ ): 3453, 3053, 2961, 2361, 1733, 1429, 1198, 1031, 736.

HRMS (ESI): $[\mathrm{M}+\mathrm{Na}]^{+}$calcd for $\mathrm{C}_{15} \mathrm{H}_{22} \mathrm{NaO}_{2} \mathrm{Si} 285.1281$, found: 285.1279.

\section{5-(Methyl(phenyl)(vinyl)silyl)pentan-2-one (3q)}

O $\mathrm{Ph}$ Me This compound was prepared according to General procedure II Me from the reaction of $\mathbf{1 q}(33.0 \mathrm{mg}, 0.2 \mathrm{mmol})$ and $\mathbf{2 b}(110.0 \mathrm{mg}$, $0.6 \mathrm{mmol}$ ). $21.8 \mathrm{mg}, 47 \%$ yield, colorless oil. $\mathrm{R}_{\mathrm{f}}=0.5$ (silica gel, petroleum ether/ethyl acetate $=10: 1)$.

${ }^{1}$ H NMR (400 MHz, CDCl 3$)$ $\delta$ 7.52-7.49 (m, 2 H), 7.37-7.34 (m, 3 H), 6.33-6.24 (m, $1 \mathrm{H}), 6.12-6.08(\mathrm{~m}, 1 \mathrm{H}), 5.80-5.74(\mathrm{~m}, 1 \mathrm{H}), 2.45(\mathrm{t}, J=7.2 \mathrm{~Hz}, 2 \mathrm{H}), 2.09$ (s, $3 \mathrm{H})$, 1.68-1.60 (m, $2 \mathrm{H}), 0.86-0.82$ (m, $2 \mathrm{H}), 0.36$ (s, $3 \mathrm{H})$.

${ }^{13}$ C NMR (100 MHz, CDCl 3$) \delta$ 209.2, 137.2, 136.5, 134.2, 133.9, 129.3, 128.0, 47.3, $30.1,18.5,14.0,-5.0$.

${ }^{29} \mathrm{Si}$ NMR (79 MHz, $\left.\mathrm{CDCl}_{3}\right): \delta-9.8$.

IR (neat, cm$^{-1}$ ): 3051, 2928, 2363, 1716, 1429, 1254, 1113, 1012, 796.

HRMS (ESI): $[\mathrm{M}+\mathrm{Na}]^{+}$calcd for $\mathrm{C}_{14} \mathrm{H}_{20} \mathrm{NaOSi} 255.1176$, found: 255.1174 .

\section{5-(Methyl(phenyl)(vinyl)silyl)pentanenitrile (3r)}

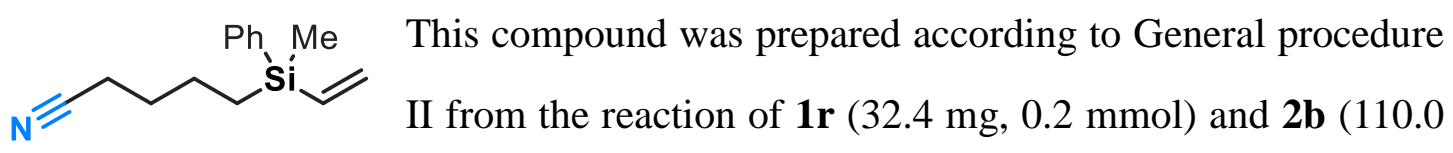


$\mathrm{mg}, 0.6 \mathrm{mmol}$ ). $14.2 \mathrm{mg}, 31 \%$ yield, colorless oil. $\mathrm{R}_{\mathrm{f}}=0.4$ (silica gel, petroleum ether/ethyl acetate $=20: 1$ ).

${ }^{1}$ H NMR (400 MHz, CDCl 3$)$ $\delta$ 7.52-7.50 (m, 2 H), 7.38-7.36 (m, 3 H), 6.33-6.24 (m, $1 \mathrm{H}), 6.14-6.09(\mathrm{~m}, 1 \mathrm{H}), 5.81-5.75(\mathrm{~m}, 1 \mathrm{H}), 2.32$ (t, $J=8.0 \mathrm{~Hz}, 2 \mathrm{H}), 1.72-1.65$ (m, 2 H), 1.56-1.46 (m, $2 \mathrm{H}), 0.88-0.84$ (m, $2 \mathrm{H}), 0.36$ (s, $3 \mathrm{H})$.

${ }^{13}$ C NMR (100 MHz, CDCl $) \delta 137.0,136.3,134.2,134.1,129.4,128.0,119.9,29.0$, $23.2,16.9,13.7,-5.0$.

${ }^{29} \mathrm{Si}$ NMR (79 MHz, CDCl 3$): ~ \delta-9.7$.

IR (neat, $\mathbf{c m}^{-1}$ ): 3051, 2949, 1590, 1405, 1253, 795, 729, 535, 469.

HRMS (APCI): $[\mathrm{M}+\mathrm{H}]^{+}$calcd for $\mathrm{C}_{14} \mathrm{H}_{20} \mathrm{NSi} 230.1360$, found: 230.1354 .

\section{6-(Methyl(phenyl)(vinyl)silyl)hexanoic acid (3s)}

O $\mathrm{Ph}$ Me This compound was prepared according to General procedure II from the reaction of $1 \mathrm{~s}(41.8 \mathrm{mg}, 0.2 \mathrm{mmol})$ and $\mathbf{2 b}$ (110.0 mg, $0.6 \mathrm{mmol}$ ). $44.5 \mathrm{mg}, 85 \%$ yield, colorless oil. $\mathrm{R}_{\mathrm{f}}=0.3$ (silica gel, ethyl acetate).

${ }^{1}$ H NMR (400 MHz, CDCl3) $\delta$ 7.54-7.51 (m, 2 H), 7.38-7.36 (m, 3 H), 6.34-6.26 (m, $1 \mathrm{H}), 6.13-6.08(\mathrm{~m}, 1 \mathrm{H}), 5.80-5.74(\mathrm{~m}, 1 \mathrm{H}), 2.33(\mathrm{t}, J=7.6 \mathrm{~Hz}, 2 \mathrm{H}), 1.68-1.60(\mathrm{~m}, 2$ H), 1.41-1.38 (m, $4 \mathrm{H}), 0.89-0.85$ (m, $2 \mathrm{H}), 0.36$ (s, $3 \mathrm{H})$.

${ }^{13}$ C NMR (100 MHz, CDCl 3$) \delta 180.4,137.6,136.9,134.2,133.6,129.2,127.9,34.1$, $33.0,24.4,14.1,-4.9$.

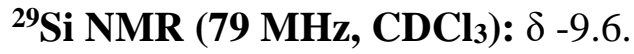

IR (neat, $\mathbf{c m}^{-1}$ ): 3052, 2928, 2850, 1709, 1407, 1252, 1113, 790, 732.

HRMS (ESI): $[\mathrm{M}+\mathrm{Na}]^{+}$calcd for $\mathrm{C}_{15} \mathrm{H}_{22} \mathrm{O}_{2} \mathrm{Si} 285.1281$, found: 285.1280 .

\section{6-(Methyl(phenyl)(vinyl)silyl)hexan-1-ol (3t)}

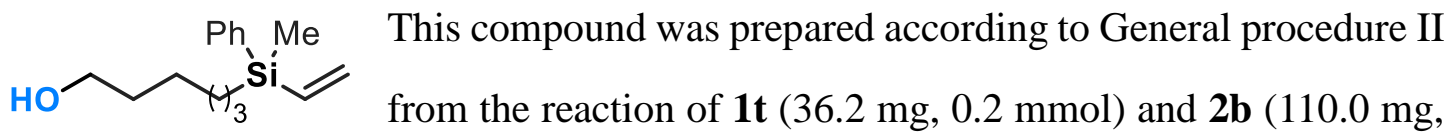
$0.6 \mathrm{mmol}$ ). $24.9 \mathrm{mg}, 51 \%$ yield, colorless oil. $\mathrm{R}_{\mathrm{f}}=0.5$ (silica gel, petroleum ether/ethyl acetate $=4: 1)$. 
${ }^{1} \mathbf{H}$ NMR (600 MHz, CDCl $)$ : $\delta$ 7.52-7.50 (m, $\left.2 \mathrm{H}\right)$, 7.36-7.35 (m, $\left.3 \mathrm{H}\right)$, 6.31-6.25 (m, $1 \mathrm{H}), 6.10-6.06(\mathrm{~m}, 1 \mathrm{H}), 5.77-5.73(\mathrm{~m}, 1 \mathrm{H}), 3.62(\mathrm{t}, J=6.6 \mathrm{~Hz}, 2 \mathrm{H}), 1.59(\mathrm{~s}, 1 \mathrm{H})$, 1.54-1.52 (m, $2 \mathrm{H}), 1.37-1.33$ (m, $6 \mathrm{H}), 0.86-0.84$ (m, $2 \mathrm{H}), 0.33$ (s, $3 \mathrm{H})$.

${ }^{13}$ C NMR (100 MHz, CDCl3): $\delta$ 137.8, 137.1, 134.2, 133.5, 129.1, 127.9, 63.2, 33.4, $32.8,25.5,23.8,14.2,-4.9$.

${ }^{29} \mathrm{Si}$ NMR (79 MHz, CDCl 3$): \delta-9.7$.

IR (neat, $\mathbf{c m}^{-1}$ ): 2954, 2927, 1732, 1715, 797, 731, 703, 659, 635.

HRMS (ESI): [M+Na] ${ }^{+}$calcd for $\mathrm{C}_{15} \mathrm{H}_{24} \mathrm{NaOSi} 271.1489$, found: 271.1489 .

6-(Methyl(phenyl)(vinyl)silyl)-N-(p-tolyl)hexanamide (3u)

II from the reaction of $1 \mathbf{u}(56.8 \mathrm{mg}, 0.2 \mathrm{mmol})$ and $\mathbf{2 b}(110.0$
$\mathrm{mg}, 0.6 \mathrm{mmol}) .54 .9 \mathrm{mg}, 78 \%$ yield, colorless oil. $\mathrm{R}_{\mathrm{f}}=0.4$ ( ilica gel, petroleum ether/ethyl acetate $=2: 1$ ).

${ }^{1}$ H NMR (400 MHz, CDCl $) \delta$ 7.53-7.51 (m, 2 H), 7.40-7.34 (m, 6 H), 7.11 (d, $J=$ 8.0 Hz, 2 H), 6.33-6.24 (m, 1 H), 6.11-6.07 (m, 1 H), 5.79-5.73 (m, 1 H), 2.31-2.27 (m, 5 H), 1.74-1.66 (m, 2 H), 1.40-1.35 (m, 4 H), 0.88-0.84 (m, 2 H), 0.35 (s, 3 H). ${ }^{13}$ C NMR (100 MHz, CDCl $) \delta 171.5,137.6,136.9,135.6,134.2,133.8,133.5$, $129.5,129.1,127.9,120.1,37.3,33.2,25.4,23.6,21.0,14.1,-4.9$.

${ }^{29} \mathrm{Si}$ NMR (79 MHz, CDCl3): $\delta-9.6$.

IR (neat, $\mathbf{c m}^{-1}$ ): 3299, 3051, 2926, 2860, 2365, 1660, 1537, 1254, 818.

HRMS (ESI): $[\mathrm{M}+\mathrm{H}]^{+}$calcd for $\mathrm{C}_{22} \mathrm{H}_{30} \mathrm{NOSi}$ 352.2091, found: 352.2090.

\section{N-methyl-N-(6-(methyl(phenyl)(vinyl)silyl)hexyl)aniline (3v)}

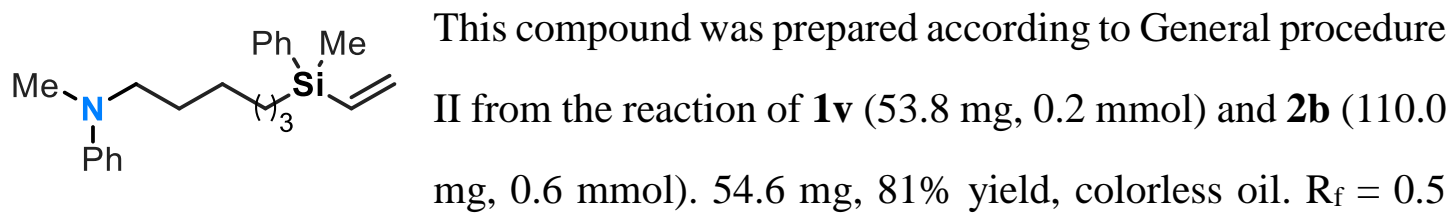

(silica gel, petroleum ether).

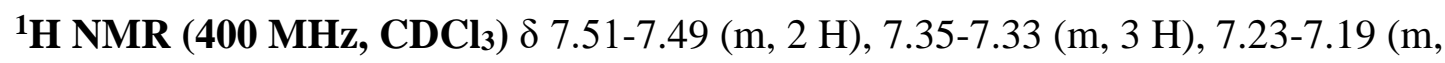
$2 \mathrm{H}), 6.68-6.64(\mathrm{~m}, 3 \mathrm{H}), 6.32-6.23$ (m, $1 \mathrm{H}), 6.09-6.05(\mathrm{~m}, 1 \mathrm{H})$, 5.77-5.71 (m, $1 \mathrm{H})$, 
$3.26(\mathrm{t}, J=7.6 \mathrm{~Hz}, 2 \mathrm{H}), 2.89$ (s, $3 \mathrm{H}), 1.54-1.51(\mathrm{~m}, 2 \mathrm{H}), 1.35-1.29(\mathrm{~m}, 6 \mathrm{H}), 0.85-$

$0.84(\mathrm{~m}, 2 \mathrm{H}) 0.33$ (s, $3 \mathrm{H})$.

${ }^{13}$ C NMR (100 MHz, CDCl $) \delta$ 149.5, 137.8, 137.1, 134.2, 133.4, 129.3, 129.1,

$127.9,115.9,112.2,52.9,38.4,33.5,26.9,26.7,23.9,14.2,-4.9$.

${ }^{29} \mathrm{Si}$ NMR (79 MHz, CDCl3): $\delta$-9.6.

IR (neat, $\mathbf{c m}^{-1}$ ): 3049, 2928, 2857, 1601, 1508, 1370, 1247, 837, 747.

HRMS (ESI): $[\mathrm{M}+\mathrm{H}]^{+}$calcd for $\mathrm{C}_{22} \mathrm{H}_{32} \mathrm{NSi} 338.2299$, found: 338.2299 .

\section{1,6-bis(methyl(phenyl)(vinyl)silyl)hexane (3w)}

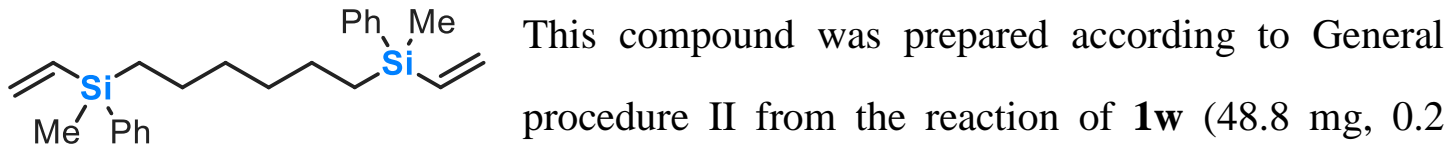
$\mathrm{mmol}$ ) and $2 \mathbf{b}(220.0 \mathrm{mg}, 1.2 \mathrm{mmol}) .68 .9 \mathrm{mg}, 91 \%$ yield, colorless oil. $\mathrm{R}_{\mathrm{f}}=0.6$ (silica gel, petroleum ether).

${ }^{1}$ H NMR (400 MHz, CDCl $)$ ) $8.51-7.49$ (m, 4 H), 7.35-7.33 (m, 6 H), 6.31-6.23 (m, $2 \mathrm{H})$, 6.09-6.04 (m, $2 \mathrm{H})$, 5.77-5.71 (m, $2 \mathrm{H}), 1.33-1.26$ (m, $8 \mathrm{H}), 0.83-0.79$ (m, $4 \mathrm{H})$, $0.32(\mathrm{~s}, 6 \mathrm{H})$.

${ }^{13}$ C NMR (100 MHz, CDCl3) $\delta 137.9,137.2,134.2,133.4,129.1,127.9,33.2,23.7$, $14.2,-4.9$

${ }^{29} \mathrm{Si}$ NMR (79 MHz, CDCl 3$): \delta-9.7$.

IR (neat, $\mathbf{c m}^{-1}$ ): 3051, 2924, 2857, 1407, 1249, 1008, 951, 837, 706.

HRMS (ESI): $[\mathrm{M}+\mathrm{K}]^{+}$calcd for $\mathrm{C}_{24} \mathrm{H}_{34} \mathrm{KSi}_{2} 417.1831$, found: 417.1826 .

\section{1,4-Bis(4-(methyl(phenyl)(vinyl)silyl)butoxy)benzene $(3 x)$}

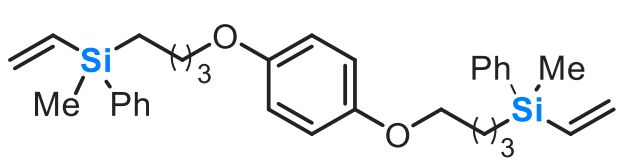

This compound was prepared according to General procedure II from the reaction of $\mathbf{1 x}$ (76.0 $\mathrm{mg}, 0.2 \mathrm{mmol})$ and $\mathbf{2 b}(220.0 \mathrm{mg}, 1.2$ mmol). $94.8 \mathrm{mg}, 92 \%$ yield, colorless oil. $\mathrm{R}_{\mathrm{f}}=0.6$ (silica gel, petroleum ether/ethyl acetate $=20: 1)$.

${ }^{1}$ H NMR (600 MHz, CDCl $)$ ) $\delta$ 7.52-7.50 (m, 4 H), 7.35-7.33 (m, $\left.6 \mathrm{H}\right), 6.78(\mathrm{~s}, 4 \mathrm{H})$, 6.31-6.25 (m, 2 H), 6.10-6.07 (m, 2 H), 5.78-5.74 (m, 2 H), 3.87 (t, J = 6.0 Hz, 4 H), 1.80-1.75 (m, 4 H), 1.54-1.49 (m, 4 H), 0.91-0.88 (m, 4 H), 0.35 (s, $6 \mathrm{H})$. 
${ }^{13}$ C NMR (100 MHz, CDCl 3$) \delta 153.3,137.5,136.8,134.2,133.6,129.2,127.9$, $115.5,68.2,33.1,20.5,14.0,-4.9$.

${ }^{29} \mathrm{Si}$ NMR (79 MHz, CDCl 3$): \delta$-9.6.

IR (neat, $\mathbf{c m}^{-1}$ ): 3051, 2943, 2868, 1593, 1508, 1429, 1228, 1113, 1027, 792, 701.

HRMS (ESI): $[\mathrm{M}+\mathrm{Na}]^{+}$calcd for $\mathrm{C}_{32} \mathrm{H}_{42} \mathrm{NaO}_{2} \mathrm{Si}_{2}$ 537.2616, found: 537.2615.

\section{Ethyl 4-((4-fluorophenyl)(methyl)(vinyl)silyl)butanoate (3y)}

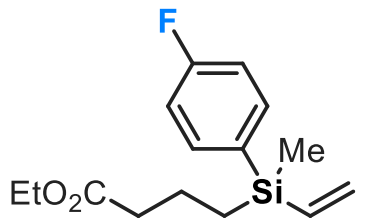

This compound was prepared according to General procedure II from the reaction of $\mathbf{1 p}(39.0 \mathrm{mg}, 0.2 \mathrm{mmol})$ and $\mathbf{2 c}(120.4$ $\mathrm{mg}, 0.6 \mathrm{mmol}) .54 .3 \mathrm{mg}$, $97 \%$ yield, colorless oil. $\mathrm{R}_{\mathrm{f}}=0.6$ ( silica gel, petroleum ether/ethyl acetate $=20: 1$ ).

${ }^{1}$ H NMR (400 MHz, CDCl3) $\delta 7.48(\mathrm{t}, J=8.0 \mathrm{~Hz}, 2 \mathrm{H}), 7.05(\mathrm{t}, J=9.6 \mathrm{~Hz}, 2 \mathrm{H})$, 6.30-6.21 (m, $1 \mathrm{H}), 6.12-6.08(\mathrm{~m}, 1 \mathrm{H}), 5.79-5.73(\mathrm{~m}, 1 \mathrm{H}), 4.14-4.09$ (q, $J=6.8 \mathrm{~Hz}$, $2 \mathrm{H}), 2.32$ (t, $J=7.2 \mathrm{~Hz}, 2 \mathrm{H}), 1.71-1.63(\mathrm{~m}, 2 \mathrm{H}), 1.24$ (t, $J=7.2 \mathrm{~Hz}, 3 \mathrm{H}), 0.87-0.84$ (m, $2 \mathrm{H}), 0.35$ (s, $3 \mathrm{H})$.

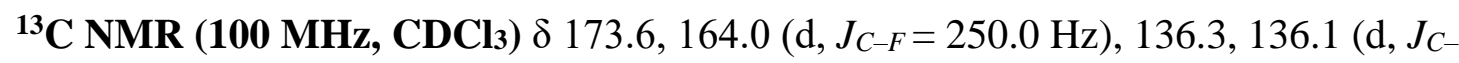
$\left.{ }_{F}=10.0 \mathrm{~Hz}\right), 134.1,132.6\left(\mathrm{~d}, J_{C-F}=4.0 \mathrm{~Hz}\right), 115.1\left(\mathrm{~d}, J_{C-F}=20.0 \mathrm{~Hz}\right), 60.3,37.8$, $19.7,14.4,14.1,-4.8$.

${ }^{19}$ F NMR (376 MHz, CDCl 3$) ~ \delta-111.9$.

${ }^{29} \mathrm{Si}$ NMR (79 MHz, CDCl3): $\delta-10.8$.

IR (neat, $\mathbf{c m}^{-1}$ ): 2947, 1735, 1590, 1500, 1374, 1165, 1105, 826, 751

HRMS (ESI): $[\mathrm{M}+\mathrm{Na}]^{+}$calcd for $\mathrm{C}_{15} \mathrm{H}_{21} \mathrm{FNaO}_{2} \mathrm{Si} 303.1187$, found: 303.1188 .

Ethyl (R)-4-(methyl(p-tolyl)(vinyl)silyl)butanoate (3z)

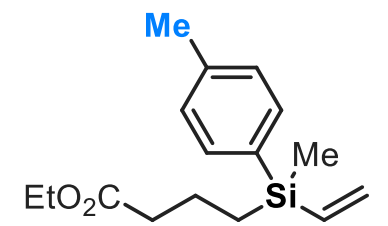

This compound was prepared according to General procedure II from the reaction of $\mathbf{1 p}(39.0 \mathrm{mg}, 0.2 \mathrm{mmol})$ and $\mathbf{2 d}(118.1$ $\mathrm{mg}, 0.6 \mathrm{mmol}) .42 .0 \mathrm{mg}, 76 \%$ yield, colorless oil. $\mathrm{R}_{\mathrm{f}}=0.6$ (silica gel, petroleum ether/ethyl acetate $=20: 1$ ).

${ }^{1}$ H NMR (400 MHz, CDCl $) \delta 7.42(\mathrm{~d}, J=7.2 \mathrm{~Hz}, 2 \mathrm{H}), 7.19(\mathrm{~d}, J=7.6 \mathrm{~Hz}, 2 \mathrm{H})$, 6.33-6.24 (m, $1 \mathrm{H}), 6.12-6.07(\mathrm{~m}, 1 \mathrm{H}), 5.80-5.74(\mathrm{~m}, 1 \mathrm{H}), 4.13(\mathrm{t}, J=6.8 \mathrm{~Hz}, 2 \mathrm{H})$, 
2.36-2.32 (m, $5 \mathrm{H}), 1.74-1.64(\mathrm{~m}, 2 \mathrm{H}), 1.26$ (t, $J=7.2 \mathrm{~Hz}, 3 \mathrm{H}), 0.90-0.86$ (m, $2 \mathrm{H})$, $0.35(\mathrm{~s}, 3 \mathrm{H})$.

${ }^{13}$ C NMR (100 MHz, CDCl $) \delta$ 173.6, 139.1, 136.7, 134.3, 133.7, 133.4, 128.8, 60.3, 38.0, 21.6, 19.8, 14.4, 14.2, -4.9.

${ }^{29} \mathrm{Si}$ NMR (79 MHz, CDCl 3$): \delta-10.0$.

IR (neat, cm$^{-1}$ ): 3446, 2945, 1735, 1602, 1374, 1254, 1197, 1107, 1031, 781.

HRMS (ESI): $[\mathrm{M}+\mathrm{Na}]^{+}$calcd for $\mathrm{C}_{16} \mathrm{H}_{24} \mathrm{NaO}_{2} \mathrm{Si} 299.1438$, found: 299.1438 .

Ethyl 4-((4-methoxyphenyl)(methyl)(vinyl)silyl)butanoate (3aa)

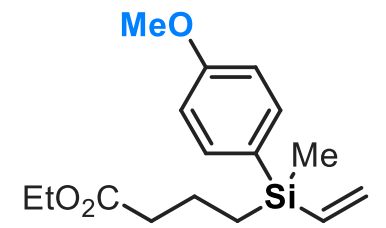

This compound was prepared according to General procedure II from the reaction of $\mathbf{1 p}(39.0 \mathrm{mg}, 0.2 \mathrm{mmol})$ and $\mathbf{2 e}(127.7$

( silica gel, petroleum ether/ethyl acetate $=20: 1$ ).

${ }^{1} \mathbf{H}$ NMR (400 MHz, CDCl $) \delta 7.44(\mathrm{~d}, J=8.0 \mathrm{~Hz}, 2 \mathrm{H}), 6.92(\mathrm{~d}, J=8.0 \mathrm{~Hz}, 2 \mathrm{H})$, 6.32-6.23 (m, 1 H), 6.11-6.06 (m, 1 H), 5.79-5.73 (m, 1 H), 4.15-4.10 (m, 2 H), 3.81 (s, $3 \mathrm{H}), 2.33(\mathrm{t}, J=8.0 \mathrm{~Hz}, 2 \mathrm{H}), 1.73-1.65(\mathrm{~m}, 2 \mathrm{H}), 1.25(\mathrm{t}, J=8.0 \mathrm{~Hz}, 3 \mathrm{H}), 0.88-$ 0.84 (m, $2 \mathrm{H}), 0.34$ (s, $3 \mathrm{H})$.

${ }^{13}$ C NMR (100 MHz, CDCl 3$) \delta 173.7,160.7,136.9,135.7,133.6,127.9,113.8,60.3$, $55.1,38.0,19.8,14.4,14.3,-4.8$.

${ }^{29}$ Si NMR (79 MHz, CDCl3): $\delta$-11.4.

IR (neat, cm-1): 2958, 2838, 1735, 1595, 1504, 1278, 1113, 1033, 751.

HRMS (ESI): $[\mathrm{M}+\mathrm{Na}]^{+}$calcd for $\mathrm{C}_{16} \mathrm{H}_{24} \mathrm{NaO}_{3} \mathrm{Si} 315.1387$, found: 315.1386 .

Ethyl 4-(methyl(o-tolyl)(vinyl)silyl)butanoate (3ab)

This compound was prepared according to General procedure
$\mathrm{mg}, 0.6 \mathrm{mmol}) .29 .3 \mathrm{mg}, 53 \%$ yield, colorless oil. $\mathrm{R}_{\mathrm{f}}=0.6$ ( silica gel, petroleum ether/ethyl acetate $=20: 1$ ).

${ }^{1} \mathbf{H}$ NMR (400 MHz, CDCl $) \delta 7.45(\mathrm{~d}, J=8.0 \mathrm{~Hz}, 1 \mathrm{H}), 7.26(\mathrm{~d}, J=8.0 \mathrm{~Hz}, 1 \mathrm{H})$, $7.15(\mathrm{t}, J=8.0 \mathrm{~Hz}, 2 \mathrm{H}), 6.37-6.28(\mathrm{~m}, 1 \mathrm{H}), 6.09-6.05(\mathrm{~m}, 1 \mathrm{H}), 5.78-5.72(\mathrm{~m}, 1 \mathrm{H})$, 
4.14-4.09 (m, 2 H), 2.42 (s, 3 H), 2.33 (m, 2 H), 1.70-1.63 (m, 2 H), 1.24 (t, J = 8.0

Hz, $3 \mathrm{H}), 0.96-0.92$ (m, $2 \mathrm{H}), 0.40$ (s, $3 \mathrm{H})$.

${ }^{13}$ C NMR (100 MHz, CDCl $) \delta 173.6,144.1,137.4,135.4,135.3,133.2,130.0$,

$129.6,125.1,60.3,37.9,23.2,19.9,14.39,14.37,-4.0$.

${ }^{29} \mathrm{Si}$ NMR (79 MHz, CDCl 3$): \delta-11.0$.

IR (neat, cm $\mathbf{c m}^{-1}$ ): 3049, 2977, 2941, 1734, 1256, 1197, 1126, 808, 745.

HRMS (ESI): [M+Na] ${ }^{+}$calcd for $\mathrm{C}_{16} \mathrm{H}_{24} \mathrm{NaO}_{2} \mathrm{Si} 299.1438$, found: 299.1437.

\section{Ethyl 4-(dimethyl(vinyl)silyl)butanoate (3ad)}

This compound was prepared according to General procedure
II from the reaction of $1 \mathbf{p}(39.0 \mathrm{mg}, 0.2 \mathrm{mmol})$ and $\mathbf{2 a}(72.4 \mathrm{mg}$, $0.6 \mathrm{mmol}$ ). $20.4 \mathrm{mg}, 51 \%$ yield, colorless oil. $\mathrm{R}_{\mathrm{f}}=0.6$ (silica gel, petroleum ether/ethyl acetate $=20: 1)$.

${ }^{1} \mathbf{H}$ NMR (400 MHz, CDCl 3$) \delta$ 6.17-6.08 (m, $\left.1 \mathrm{H}\right)$, 5.97-5.93 (m, $\left.1 \mathrm{H}\right)$, 5.70-5.64 (m, $1 \mathrm{H}), 4.15-4.09(\mathrm{~m}, 2 \mathrm{H}), 2.31(\mathrm{t}, J=8.0 \mathrm{~Hz}, 2 \mathrm{H}), 1.68-1.60(\mathrm{~m}, 2 \mathrm{H}), 1.25(\mathrm{t}, J=8.0$ Hz, $3 \mathrm{H}), 0.61-0.56(\mathrm{~m}, 2 \mathrm{H}), 0.06$ (s, $6 \mathrm{H})$.

${ }^{13}$ C NMR (100 MHz, CDCl 3$) \delta 173.8,138.8,131.9,60.3,38.1,19.8,15.3,14.4,-3.4$. ${ }^{29} \mathrm{Si}$ NMR (79 MHz, CDCl 3$): \delta-5.3$.

IR (neat, $\mathbf{c m}^{-1}$ ): 2967, 2929, 2863, 1736, 1196, 1029, 802, 747, 672.

HRMS (ESI): $[\mathrm{M}+\mathrm{Na}]^{+}$calcd for $\mathrm{C}_{10} \mathrm{H}_{20} \mathrm{NaO}_{2} \mathrm{Si} 223.1125$, found: 223.1125 .

\section{Ethyl 4-(methyl(octyl)(vinyl)silyl)butanoate (3ae)}

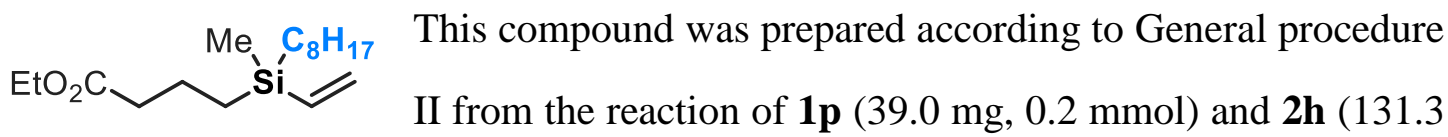
$\mathrm{mg}, 0.6 \mathrm{mmol}$ ). $52.6 \mathrm{mg}, 88 \%$ yield, colorless oil. $\mathrm{R}_{\mathrm{f}}=0.6$ (silica gel, petroleum ether/ethyl acetate $=20: 1$ )

${ }^{1}$ H NMR (400 MHz, CDCl 3 ) $\delta$ 6.14-6.05 (m, 1 H), 5.98-5.94 (m, 1 H), 5.69-5.63 (m, $1 \mathrm{H}), 4.14-4.09(\mathrm{~m}, 2 \mathrm{H}), 2.30(\mathrm{t}, J=8.0 \mathrm{~Hz}, 2 \mathrm{H}), 1.67-1.59(\mathrm{~m}, 2 \mathrm{H}), 1.26-1.24(\mathrm{~m}$, $15 \mathrm{H}), 0.87$ (t, $J=8.0 \mathrm{~Hz}, 3 \mathrm{H}), 0.60-0.56(\mathrm{~m}, 4 \mathrm{H}), 0.04(\mathrm{~s}, 3 \mathrm{H})$.

${ }^{13}$ C NMR (100 MHz, CDCl 3$) \delta 173.8, ~ 138.0,132.3,60.2,38.1,33.8,32.1,29.43$, $29.40,23.9,22.8,19.8,14.4,14.2,13.9,-5.4$. 
${ }^{29} \mathrm{Si}$ NMR (79 MHz, $\left.\mathrm{CDCl}_{3}\right): \delta-5.6$.

IR (neat, $\mathbf{c m}^{-1}$ ): 3455, 2924, 2857, 1739, 1374, 1010, 951, 803, 755.

HRMS (ESI): $[\mathrm{M}+\mathrm{Na}]^{+}$calcd for $\mathrm{C}_{17} \mathrm{H}_{34} \mathrm{NaO}_{2} \mathrm{Si}$ 321.2220, found: 321.2219 .

Ethyl 4-(benzyl(methyl)(vinyl)silyl)butanoate (3af)

$\mathrm{EtO}_{2} \mathrm{C} \_\mathrm{Me}$ This compound was prepared according to General procedure II from the reaction of $\mathbf{1 p}(39.0 \mathrm{mg}, 0.2 \mathrm{mmol})$ and $\mathbf{2 i}(118.1$ $\mathrm{mg}, 0.6 \mathrm{mmol}$ ). 53.0mg, $96 \%$ yield, colorless oil. $\mathrm{R}_{\mathrm{f}}=0.6$ (silica gel, petroleum ether/ethyl acetate $=20: 1$ ).

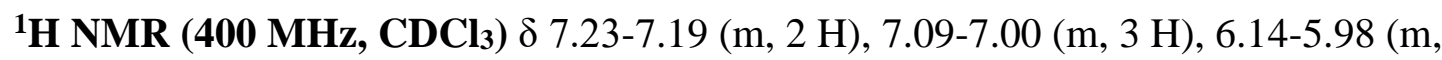
$2 \mathrm{H})$, 5.71-5.65 (m, $1 \mathrm{H}), 4.15-4.10(\mathrm{~m}, 2 \mathrm{H}), 2.30(\mathrm{t}, J=8.0 \mathrm{~Hz}, 2 \mathrm{H}), 2.17(\mathrm{~s}, 2 \mathrm{H})$, 1.68-1.69 (m, $2 \mathrm{H}), 1.25(\mathrm{t}, J=8.0 \mathrm{~Hz}, 3 \mathrm{H}), 0.63-0.58$ (m, $2 \mathrm{H}), 0.05$ (s, $3 \mathrm{H})$.

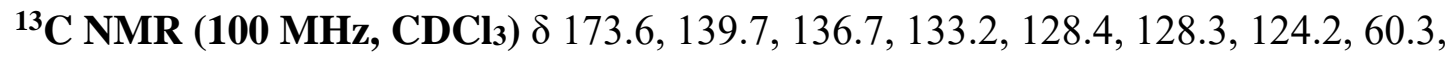
38.0, 24.3, 19.6, 14.4, 13.3, -5.7.

${ }^{29} \mathrm{Si}$ NMR (79 MHz, CDCl 3$): ~ \delta-6.4$.

IR (neat, $\mathbf{c m}^{-1}$ ): 3029, 2945, 1735, 1494, 1198, 1031, 820, 770, 699.

HRMS (ESI): $[\mathrm{M}+\mathrm{Na}]^{+}$calcd for $\mathrm{C}_{16} \mathrm{H}_{24} \mathrm{NaO}_{2} \mathrm{Si} 299.1438$, found: 299.1437.

\section{Ethyl 4-(methyl(pent-4-en-1-yl)(vinyl)silyl)butanoate (3ag)}

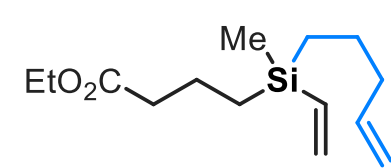

This compound was prepared according to General procedure II from the reaction of $\mathbf{1 p}(39.0 \mathrm{mg}, 0.2 \mathrm{mmol})$ and $\mathbf{2 j}$ (104.8 $\mathrm{mg}, 0.6 \mathrm{mmol}) .42 .2 \mathrm{mg}, 83 \%$ yield, colorless oil. $\mathrm{R}_{\mathrm{f}}=0.6$

( silica gel, petroleum ether/ethyl acetate $=20: 1$ ).

${ }^{1}$ H NMR (400 MHz, CDCl3) $\delta$ 6.14-6.05 (m, 1 H), 5.99-5.94 (m, 1 H), 5.83-5.72 (m, $1 \mathrm{H}), 5.70-5.64$ (m, $1 \mathrm{H}), 5.01-4.92(\mathrm{~m}, 2 \mathrm{H}), 4.14-4.09$ (m, $2 \mathrm{H}), 2.30$ (t, $J=8.0 \mathrm{~Hz}, 2$ H), 2.08-2.03 (m, 2 H), 1.67-1.59 (m, $2 \mathrm{H}), 1.42-1.34 \mathrm{~m}, 2 \mathrm{H}), 1.24$ (t, $J=8.0 \mathrm{~Hz}, 3$ H), 0.61-0.56 (m, $4 \mathrm{H}), 0.05$ (s, $3 \mathrm{H})$.

${ }^{13}$ C NMR (100 MHz, CDCl3) $\delta 173.7,138.9,137.7,114.7,60.2,38.1,37.7,23.4$, $19.8,14.4,13.8,13.4,-5.5$.

${ }^{29} \mathrm{Si}$ NMR (79 MHz, CDCl 3$): \delta-9.6$.

IR (neat, cm$^{-1}$ ): 3051, 2980, 2928, 1737, 1640, 1407, 1197, 1124, 1031, 807. 
HRMS (ESI): $[\mathrm{M}+\mathrm{Na}]^{+}$calcd for $\mathrm{C}_{14} \mathrm{H}_{26} \mathrm{NaO}_{2} \mathrm{Si} 277.1594$, found: 277.1595.

\section{Ethyl 4-(cyclopropyl(methyl)(vinyl)silyl)butanoate (3ah)}

Me $>$ This compound was prepared according to General procedure

$\mathrm{EtO}_{2} \mathrm{C} \smile \mathrm{Si} \_$II from the reaction of $\mathbf{1 p}(39.0 \mathrm{mg}, 0.2 \mathrm{mmol})$ and $\mathbf{2 k}(88.0 \mathrm{mg}$, $0.6 \mathrm{mmol}$ ). $43.4 \mathrm{mg}, 96 \%$ yield, colorless oil. $\mathrm{R}_{\mathrm{f}}=0.6$ (silica gel, petroleum ether/ethyl acetate $=20: 1)$.

${ }^{1}$ H NMR (400 MHz, CDCl3) $\delta$ 6.08-5.94 (m, 2 H), 5.75-5.69 (m, 1 H), 4.14-4.09 (m, $2 \mathrm{H}), 2.32(\mathrm{t}, J=8.0 \mathrm{~Hz}, 2 \mathrm{H}), 1.73-1.65(\mathrm{~m}, 2 \mathrm{H}), 1.24(\mathrm{t}, J=8.0 \mathrm{~Hz}, 3 \mathrm{H}), 0.62-0.53$ (m, $4 \mathrm{H}), 0.20-0.18(\mathrm{~m}, 2 \mathrm{H}),-0.05(\mathrm{~s}, 3 \mathrm{H}),(-0.38)-(-0.46)(\mathrm{m}, 1 \mathrm{H})$. ${ }^{13}$ C NMR (100 MHz, CDCl3) $\delta 173.8,136.7,132.9,60.2,38.1,19.8,14.4,13.8,1.1$, $1.0,-6.7,-6.8$

${ }^{29} \mathrm{Si}$ NMR (79 MHz, $\left.\mathrm{CDCl}_{3}\right): \delta-4.3$.

IR (neat, $\mathbf{c m}^{-1}$ ): 3051, 2932, 2857, 1646, 1429, 1250, 1116, 1034, 794, 701.

HRMS (ESI): $[\mathrm{M}+\mathrm{Na}]^{+}$calcd for $\mathrm{C}_{12} \mathrm{H}_{22} \mathrm{NaO}_{2} \mathrm{Si} 249.1281$, found: 249.1281 .

\section{Ethyl 4-(methyl(phenylethynyl)(vinyl)silyl)butanoate (3ai)}

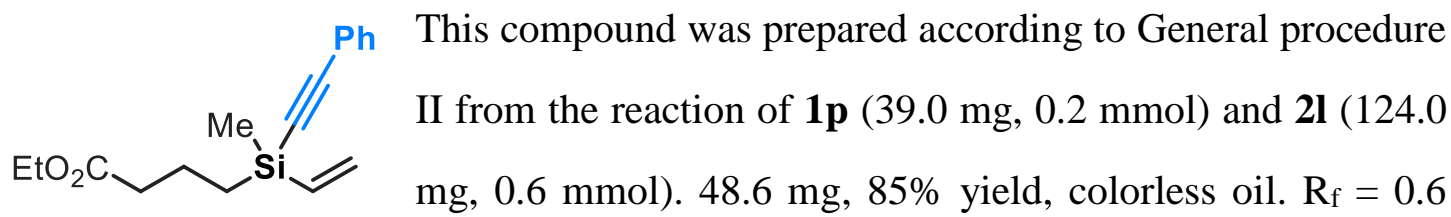

(silica gel, petroleum ether/ethyl acetate $=20: 1$ ).

${ }^{1}$ H NMR (400 MHz, CDCl $) \delta$ 7.49-7.47 (m, 2 H), 7.32-7.29 (m, 3 H), 6.21-6.06 (m, 2 H), 5.97-5.91 (m, 1 H), 4.15-4.10 (m, 2 H), 2.39 (t, $J=8.0$ Hz, 2 H), 1.84-1.76 (m, 2 $\mathrm{H}), 1.25(\mathrm{t}, J=8.0 \mathrm{~Hz}, 3 \mathrm{H}), 0.81-0.77(\mathrm{~m}, 2 \mathrm{H}), 0.31(\mathrm{~s}, 3 \mathrm{H})$.

${ }^{13}$ C NMR (100 MHz, CDCl $) \delta$ 173.6, 135.1, 134.2, 132.2, 128.8, 128.3, 123.0, $107.1,90.8,60.3,37.6,19.7,14.9,14.4,-3.3$.

${ }^{29} \mathrm{Si}$ NMR (79 MHz, CDCl 3$): \delta-23.5$.

IR (neat, cm$^{-1}$ ): 3056, 2963, 2160, 1737, 1489, 1256, 1198, 1029, 837.

HRMS (ESI): $[\mathrm{M}+\mathrm{Na}]^{+}$calcd for $\mathrm{C}_{17} \mathrm{H}_{22} \mathrm{NaO}_{2} \mathrm{Si} 309.1281$, found: 309.1281 . 


\section{Ethyl (E)-4-(dimethyl(styryl)silyl)butanoate (3aj)}

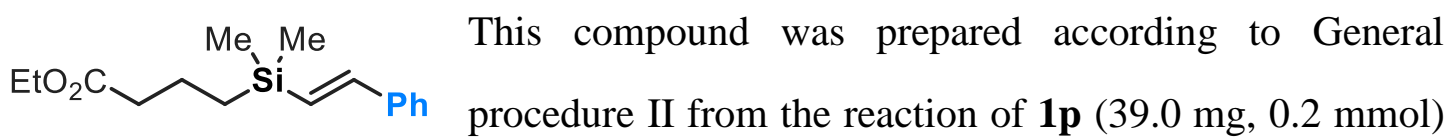

and $2 \mathrm{~m}(118.1 \mathrm{mg}, 0.6 \mathrm{mmol}) .33 .7 \mathrm{mg}, 61 \%$ yield, colorless oil. $\mathrm{R}_{\mathrm{f}}=0.5$ (silica gel, petroleum ether/ethyl acetate $=20: 1$ ).

${ }^{1}$ H NMR (400 MHz, CDCl 3$) \delta$ 7.44-7.42 (m, 2 H), 7.34-7.29 (m, 2 H), 7.26-7.23 (m, $1 \mathrm{H}), 6.89(\mathrm{~d}, J=20.0 \mathrm{~Hz}, 1 \mathrm{H}), 6.45(\mathrm{~d}, J=20.0 \mathrm{~Hz}, 1 \mathrm{H}), 4.15-4.10(\mathrm{~m}, 2 \mathrm{H}), 2.34$ (t, $J=8.0 \mathrm{~Hz}, 2 \mathrm{H}), 1.73-1.65(\mathrm{~m}, 2 \mathrm{H}), 1.25(\mathrm{t}, J=8.0 \mathrm{~Hz}, 3 \mathrm{H}), 0.69-0.65(\mathrm{~m}, 2 \mathrm{H})$, $0.16(\mathrm{~s}, 6 \mathrm{H})$.

${ }^{13}$ C NMR (100 MHz, CDCl 3$) \delta 173.8,144.5,138.4,128.7,128.2,128.0,126.5,60.3$, $38.1,19.9,15.7,14.4,-3.0$.

${ }^{29} \mathrm{Si}$ NMR (79 MHz, CDCl 3$): \delta 0.0$.

IR (neat, $\mathbf{c m}^{-1}$ ): 2956, 2158, 1735, 1196, 913, 841, 777, 745, 691.

HRMS (ESI): $[\mathrm{M}+\mathrm{Na}]^{+}$calcd for $\mathrm{C}_{16} \mathrm{H}_{24} \mathrm{NaO}_{2} \mathrm{Si} 299.1438$, found: 299.1437 .

\section{Hexyldimethyl(vinyl)silane (3ak)}

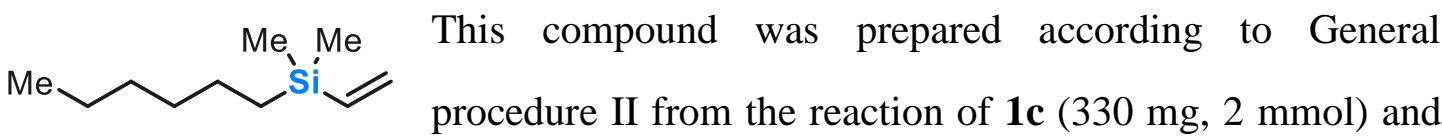

2a (724 mg, $6 \mathrm{mmol}$ ). $258 \mathrm{mg}, 76 \%$ yield, colorless oil. $\mathrm{R}_{\mathrm{f}}=0.6$ (silica gel, petroleum ether).

${ }^{1}$ H NMR (400 MHz, CDCl 3$) \delta$ 6.19-6.10 (m, 1 H), 5.96-5.92 (m, 1 H), 5.69-5.63 (m, $1 \mathrm{H}), 1.31-1.27(\mathrm{~m}, 8 \mathrm{H}), 0.88(\mathrm{t}, J=8.0 \mathrm{~Hz}, 3 \mathrm{H}), 0.58-0.54(\mathrm{~m}, 2 \mathrm{H}), 0.05(\mathrm{~s}, 6 \mathrm{H})$.

${ }^{13}$ C NMR (100 MHz, CDCl3) $\delta$ 139.6, 131.4, 33.4, 31.8, 23.9, 22.8, 15.5, 14.3, -3.2.

${ }^{29} \mathrm{Si}$ NMR (79 MHz, CDCl 3$): \delta-5.3$.

IR (neat, $\mathbf{c m}^{-1}$ ): 2966, 2929, 2865, 1406, 1251, 952, 913, 745, 666.

HRMS (APCI): $[\mathrm{M}+\mathrm{H}]^{+}$calcd for $\mathrm{C}_{10} \mathrm{H}_{23} \mathrm{Si} 171.1564$, found: 171.1561 . 


\section{Synthetic Application}

\subsection{Silicon incorporation into biologically active molecules}

6-(Methyl(phenyl)(vinyl)silyl)hexyl 2-(1-(4-chlorobenzoyl)-5-methoxy-2-methyl$1 H$-indol-3-yl)acetate (4)

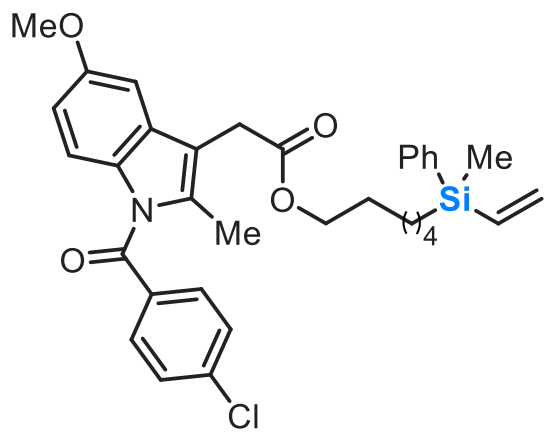

This compound was prepared according to General procedure II from the reaction of $\mathbf{1 y}(52.1 \mathrm{mg}, 0.1$ $\mathrm{mmol})$ and $2 \mathrm{~b}(55.0 \mathrm{mg}, 0.3 \mathrm{mmol}) .49 .4 \mathrm{mg}, 84 \%$ yield, colorless oil. $\mathrm{R}_{\mathrm{f}}=0.5$ (silica gel, petroleum ether/ethyl acetate $=5: 1$ ).

${ }^{1} \mathrm{H}$ NMR (400 MHz, CDCl3) $\delta 7.65(\mathrm{~d}, J=8.0 \mathrm{~Hz}$, $2 \mathrm{H}), 7.50-7.45(\mathrm{~m}, 4 \mathrm{H}), 7.35-7.34(\mathrm{~m}, 3 \mathrm{H}), 6.96(\mathrm{~m}, 1 \mathrm{H}), 6.86(\mathrm{~d}, J=8.0 \mathrm{~Hz}, 1 \mathrm{H})$, 6.67-6.64 (m, $1 \mathrm{H})$, 6.31-6.22 (m, $1 \mathrm{H}), 6.09-6.05$ (m, $1 \mathrm{H})$, 5.77-5.71 (m, $1 \mathrm{H}), 4.07$ $(\mathrm{d}, J=8.0 \mathrm{~Hz}, 2 \mathrm{H}), 3.81(\mathrm{~s}, 3 \mathrm{H}), 3.64(\mathrm{~s}, 2 \mathrm{H}), 2.38(\mathrm{~s}, 3 \mathrm{H}), 1.61-1.56(\mathrm{~m}, 2 \mathrm{H})$, 1.30 (m, $6 \mathrm{H}), 0.83-0.81(\mathrm{~m}, 2 \mathrm{H}), 0.32$ (s, $3 \mathrm{H})$.

${ }^{13}$ C NMR (100 MHz, CDCl $) \delta$ 171.1, 168.4, 156.2, 137.0, 134.2, 134.1, 133.5, 131.3, $129.2,129.1,127.9,115.1,112.9,111.8,101.5,65.3,55.8,33.3,30.6,28.7,25.7,23.8$, $14.2,13.5,-4.9$.

${ }^{29} \mathrm{Si}$ NMR (79 MHz, CDCl3): $\delta-9.7$.

IR (neat, $\mathbf{c m}^{-1}$ ): 2930, 2365, 1735, 1687, 1478, 1320, 1068, 796, 755.

HRMS (ESI): $[\mathrm{M}+\mathrm{Na}]^{+}$calcd for $\mathrm{C}_{34} \mathrm{H}_{38} \mathrm{ClNNaO}_{4} \mathrm{Si}$ 610.2151, found: 610.2149 .

Methyl(phenyl)(4-((2,2,7,7-tetramethyltetrahydro-3aH-bis([1,3]dioxolo)[4,5b:4',5'-d]pyran-3a-yl)methoxy)butyl)(vinyl)silane (5)<smiles>C/C=C\[Si](C)(C)c1ccccc1</smiles>

petroleum ether/ethyl acetate $=20: 1)$.
This compound was prepared according to General procedure II from the reaction of $\mathbf{1 z}(40.0$ $\mathrm{mg}, 0.1 \mathrm{mmol})$ and $\mathbf{2 b}(55.0 \mathrm{mg}, 0.3 \mathrm{mmol}) .41 .7$ mg, $90 \%$ yield, colorless oil. $\mathrm{R}_{\mathrm{f}}=0.4$ (silica gel, 
${ }^{1}$ H NMR (400 MHz, CDCl$)$ ) $\delta$ 7.52-7.50 (m, 2 H), 7.37-7.33 (m, 3 H), 6.32-6.24 (m, $1 \mathrm{H}), 6.10-6.06(\mathrm{~m}, 1 \mathrm{H}), 5.78-5.72(\mathrm{~m}, 1 \mathrm{H}), 4.61-4.58(\mathrm{~m}, 1 \mathrm{H}), 4.38(\mathrm{~d}, J=4.0 \mathrm{~Hz}$, $1 \mathrm{H}), 4.24-4.22(\mathrm{~m}, 1 \mathrm{H}), 3.93-3.89$ (m, $1 \mathrm{H}), 3.74-3.71(\mathrm{~m}, 1 \mathrm{H}), 3.58-3.42(\mathrm{~m}, 4 \mathrm{H})$, 1.66-1.59 (m, 2 H), 1.53 (s, 3 H), 1.47 (s, 3 H), 1.45-1.40 (m, 2 H), 1.39 (s, 3 H), 1.34 (s, $3 \mathrm{H}), 0.88-0.84(\mathrm{~m}, 2 \mathrm{H}), 0.34$ (s, $3 \mathrm{H})$.

${ }^{13}$ C NMR (100 MHz, CDCl3) $\delta$ 137.6, 136.9, 134.2, 133.5, 129.1, 127.9, 109.0, 108.6, 102.8, 72.2, 71.6, 71.2, 70.4, 70.1, 61.1, 33.4, 26.7, 26.0, 25.4, 24.2, 20.5, 14.1, -5.0 .

${ }^{29} \mathrm{Si}$ NMR (79 MHz, CDCl 3$): \delta-9.6$.

IR (neat, $\mathbf{c m}^{-1}$ ): 3051, 2993, 2939, 2871, 1636, 1381, 1252, 1072,701

HRMS (ESI): $[\mathrm{M}+\mathrm{Na}]^{+}$calcd for $\mathrm{C}_{25} \mathrm{H}_{38} \mathrm{NaO}_{6} \mathrm{Si} 485.2330$, found: 485.2328.

\subsection{Immobilization of 3ak onto glass surface}

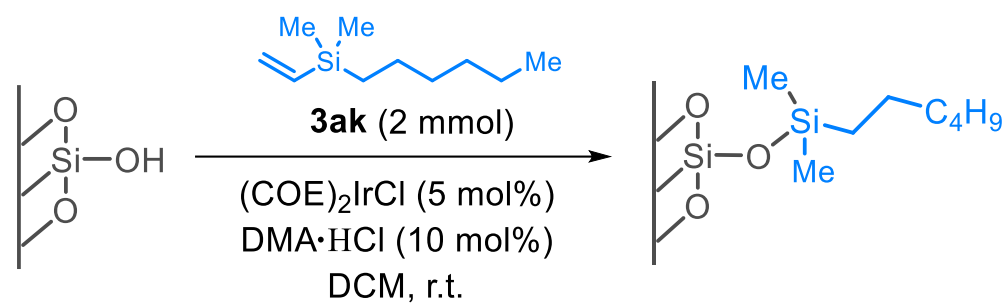

The Surface Modification of Glass: ${ }^{13}$ Glass slide $\left(1.2 \times 1.2 \mathrm{~cm}^{2}\right)$ was submerged in $60 \% \mathrm{HNO}_{3}$, and the solution was sonicated for 5 minutes. Then, the slide was treated in Piranha solution $\left(\mathrm{H}_{2} \mathrm{SO}_{4}: \mathrm{H}_{2} \mathrm{O}_{2}=7: 3,10 \mathrm{~mL}\right)$ at room temperature for 30 minutes. After washing with distilled water and methanol and drying in vacuum, glass slide was treated with a solution of alkylsilane 3ak (2 mmol), [(COE $\left.)_{2} \mathrm{IrCl}\right]_{2}(22.4 \mathrm{mg}, 5 \mathrm{~mol} \%)$, and DMA $\cdot \mathrm{HCl}(12.3 \mathrm{mg}, 10 \mathrm{~mol} \%)$ in DCM (1.0 mL). The solution was shaken for 12 hours. The glass slide was washed with DCM and methanol. After drying in vacuum, it was used for the measurement of the contact angle of the glass surface. 
The contact angle the bare surface is $23.9^{\circ}$

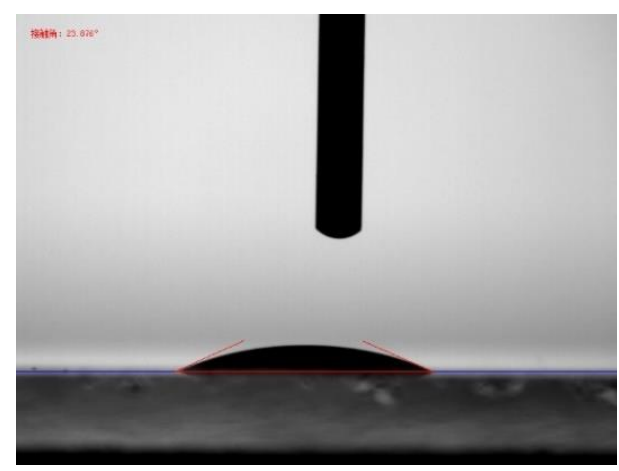

The contact angle for immobilized surface is $105.3^{\circ}$

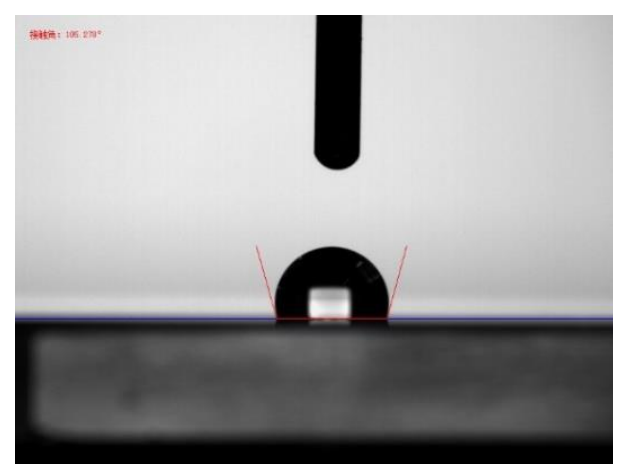

\section{Mechanistic Investigation}

\subsection{The selectivity of $1 \mathrm{~d}$ and $2 \mathrm{k}$ in the initial reaction with $\mathrm{Ni}(0)$}
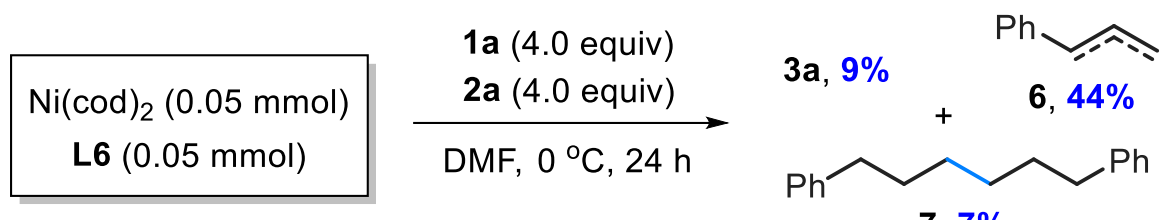

$7,7 \%$

General procedure: The procedure was conducted in an argon-filled glove box. To a reaction tube equipped with a magnetic stir bar was added $\mathrm{Ni}(\operatorname{cod})_{2}(13.7 \mathrm{mg}, 0.05$ mmol), ligand $\mathbf{L 6}$ (14.6 mg, $0.05 \mathrm{mmol}$ ). A solution of alkyl bromide 1a (39.6 mg, 0.2 mmol) and chlorosilane $2 \mathrm{a}(24.0 \mathrm{mg}, 0.2 \mathrm{mmol})$ in DMF (1.0 mL) was added. The reaction tube was sealed and removed from the glove box. The reaction mixture was stirred at $0{ }^{\circ} \mathrm{C}$ for $24 \mathrm{~h}$. The reaction mixture was diluted with ethyl acetate $(20.0 \mathrm{~mL})$, washed with water $(5.0 \mathrm{~mL})$, brine $(5.0 \mathrm{~mL})$, and dried over anhydrous $\mathrm{Na}_{2} \mathrm{SO}_{4}$. A 0.4 $\mathrm{mL}$ of solution was collected and diluted with ethyl acetate $(2 \mathrm{~mL})$, and it was analyzed by GC-MS and GC. The yield was determined versus the internal standard (dodecane). 


\subsection{Radical clock experiment}

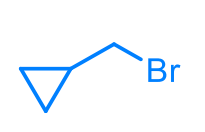

$8(0.2 \mathrm{mmol})$

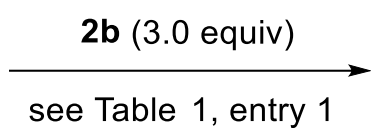

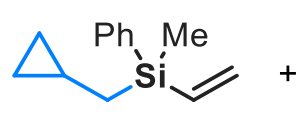

$9,0 \%$

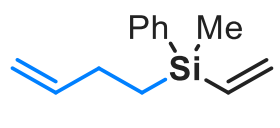

$10,34 \%$

General procedure: The procedure was conducted in an argon-filled glove box. To a reaction tube equipped with a magnetic stir bar was added $\mathrm{NiCl}_{2}(2.6 \mathrm{mg}, 0.02 \mathrm{mmol})$, 2,9-di- ${ }^{-}$bu-phen $(5.8 \mathrm{mg}, 0.02 \mathrm{mmol})$, and Mn (22.0 mg, $\left.0.4 \mathrm{mmol}\right)$. A solution of alkyl bromide 8 (26.8 mg, $0.2 \mathrm{mmol}$ ) and chlorosilane $\mathbf{2 b}$ (109 mg, $0.6 \mathrm{mmol}$ ) in DMF $(1.0 \mathrm{~mL})$ was added. The reaction tube was sealed and removed from the glove box. The reaction mixture was stirred at $0{ }^{\circ} \mathrm{C}$ for $24 \mathrm{~h}$. The reaction was quenched with water $(20.0 \mathrm{~mL})$, and the mixture solution was extracted with ethyl acetate $(3 \times 15.0 \mathrm{~mL})$. The combined organic layers were washed with water, brine, dried over anhydrous $\mathrm{Na}_{2} \mathrm{SO}_{4}$. A $0.4 \mathrm{~mL}$ of solution was collected and diluted with ethyl acetate $(1 \mathrm{~mL})$, and it was analyzed by GC-MS. The rest was concentrated in vacuum, and the residue was purified by flash chromatography on silica gel $(\mathrm{Rf}=0.6$, petroleum ether) to afford compound 10 in $34 \%$ yield $(13.8 \mathrm{mg})$ as a colorless oil.

\section{But-3-en-1-yl(methyl)(phenyl)(vinyl)silane (10)}

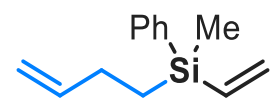

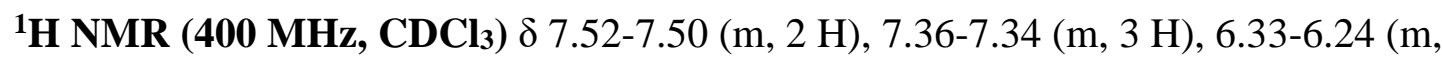
$1 \mathrm{H})$, 6.11-6.07 (m, $1 \mathrm{H})$, 5.92-5.82 (m, $1 \mathrm{H})$, 5.79-5.73 (m, $1 \mathrm{H})$, 5.00-4.87 (m, $2 \mathrm{H})$, 2.12-2.06 (m, $2 \mathrm{H}), 0.97-0.93$ (m, $2 \mathrm{H}), 0.35$ (s, $3 \mathrm{H})$.

${ }^{13}$ C NMR (100 MHz, CDCl $) \delta 141.5,137.4,136.8,134.3,133.7,129.2,128.0$, $113.1,27.9,13.4,-4.8$.

${ }^{29} \mathrm{Si}$ NMR (79 MHz, $\left.\mathrm{CDCl}_{3}\right): \delta-9.4$.

IR (neat, $\mathbf{c m}^{-1}$ ): 3069, 3050, 2961, 2915, 1111, 911, 792, 736, 698.

HRMS (APCI): $[\mathrm{M}+\mathrm{H}]^{+}$calcd for $\mathrm{C}_{13} \mathrm{H}_{19} \mathrm{Si} 203.1251$, found: 203.1248 . 


\section{References}

(1) Armarego, W. L. F.; Chai, C. C. L. Purification of laboratory chemicals, 5th ed., Butterworth- Heinemann, 2003.

(2) Newton, J. J.; Jelier, B. J.; Meanwell, M.; Martin, R. E.; Britton, R.; Friesen, C. M. Quaternary Ammonium Trifluoromethoxide Salts as Stable Sources of Nucleophilic $\mathrm{OCF}_{3}$ Org. Lett. 2020, 22, 1785.

(3) Chatterjee, I.; Porwal, D.; Oestreich, M. B( $\left.\mathrm{C}_{6} \mathrm{~F}_{5}\right)_{3}$-Catalyzed Chemoselective Defunctionalization of Ether-Containing Primary Alkyl Tosylates with Hydrosilanes. Angew. Chem. Int. Ed. 2017, 56, 3389.

(4) Sonnenberger, S.; Eichner, A.; Schmitt, T.; Hauß, T.; Lange, S.; Langner, A.; Neubert, R. H. H.; Dobner, B. Synthesis of specific deuterated derivatives of the long chained stratum corneum lipids [EOS] and [EOP] and characterization using neutron scattering. J Label Compd Radiopharm. 2017, 60, 316

(5) Brunel, F.; Lautard, C.; Garzino, F.; Giorgio, S.; Raimundo, J. M.; Bolla, J. M.; Camplo, M. Antibacterial activities of fluorescent nano assembled triphenylamine phosphonium ionic liquids. Bioorg. Med. Chem. Lett. 2016, 26, 3770.

(6) Chang, J.; Zhang, S. J.; Jiang, Y. W.; Xu, L.; Yu, J. M.; Zhou, W. J.; Sun, X. Design, Synthesis, and Antibacterial Activity of Demethylvancomycin Analogues against Drug-Resistant Bacteria. ChemMedChem 2013, 8, 976

(7) I Nguyen, C.; Ruda, G. F.; Schipani, A.; Kasinathan, G.; Leal, I.; Musso-Buendia, A.; Kaiser, M.; Brun, R.; Ruiz-Pe'rez, L. M.; Sahlberg, B.; Johansson, N. G.; Gonza'lez-Pacanowska, D.; Gilbert, I. H. Acyclic Nucleoside Analogues as Inhibitors of Plasmodium falciparum dUTPase. J. Med. Chem. 2006, 49, 4183.

(8) Yan, X. B.; Li, C. L.; Jin, W. J.; Guo, P.; Shu, X. Z. Reductive coupling of benzyl oxalates with highly functionalized alkyl bromides by nickel catalysis. Chem. Sci. 2018, 9, 4529.

(9) Lin, Q.; Fan, Y. Q.; Mao, P. P.; Liu, L.; Liu, J.; Zhang, Y. M.; Yao, H.; Wei, T. B. Pillar[5]arene-Based Supramolecular Organic Framework with Multi-Guest Detection and Recyclable Separation Properties. Chem. Eur. J. 2018, 24, 777.

(10) Simpson, P. V.; Casari, I.; Paternoster, S.; Skelton, B. W.; Falasca, M.; Massi, M. Defining the Anti-Cancer Activity of Tricarbonyl Rhenium Complexes: Induction of G2/M Cell Cycle Arrest and Blockade of Aurora-A Kinase Phosphorylation. Chem. Eur. J. 2017, 23, 6518

(11) Li, Y.; Chen, S.; Wang, M.; Jiang, X. Sodium Dithionite-Mediated Decarboxylative Sulfonylation: Facile Access to Tertiary Sulfones. Angew. Chem. Int. Ed. 2020, 59, 8907.

(12) Duan, J.; Wang, K.; Xu, G. L.; Kang, S. L.; Qi, L. L.; Liu, X. Y.; Shu, X. Z. CrossElectrophile $\mathrm{C}\left(\mathrm{sp}^{2}\right)-$ Si Coupling of Vinyl Chlorosilanes. Angew. Chem. Int. Ed. 2020, 59, 23083.

(13) Park, J. W.; Jun, C. Transition-Metal-Catalyzed Immobilization of Organic Functional Groups onto Solid Supports through Vinylsilane Coupling Reactions. J. Am. Chem. Soc. 2010, 132, 7268. 


\section{Copies of NMR Spectra}

2h; ${ }^{1} \mathrm{H}$ NMR (400 MHz, $\left.\mathrm{CDCl}_{3}\right) ;{ }^{13} \mathrm{C}$ NMR (100 MHz, $\left.\mathrm{CDCl}_{3}\right)$

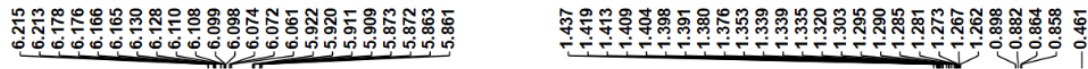
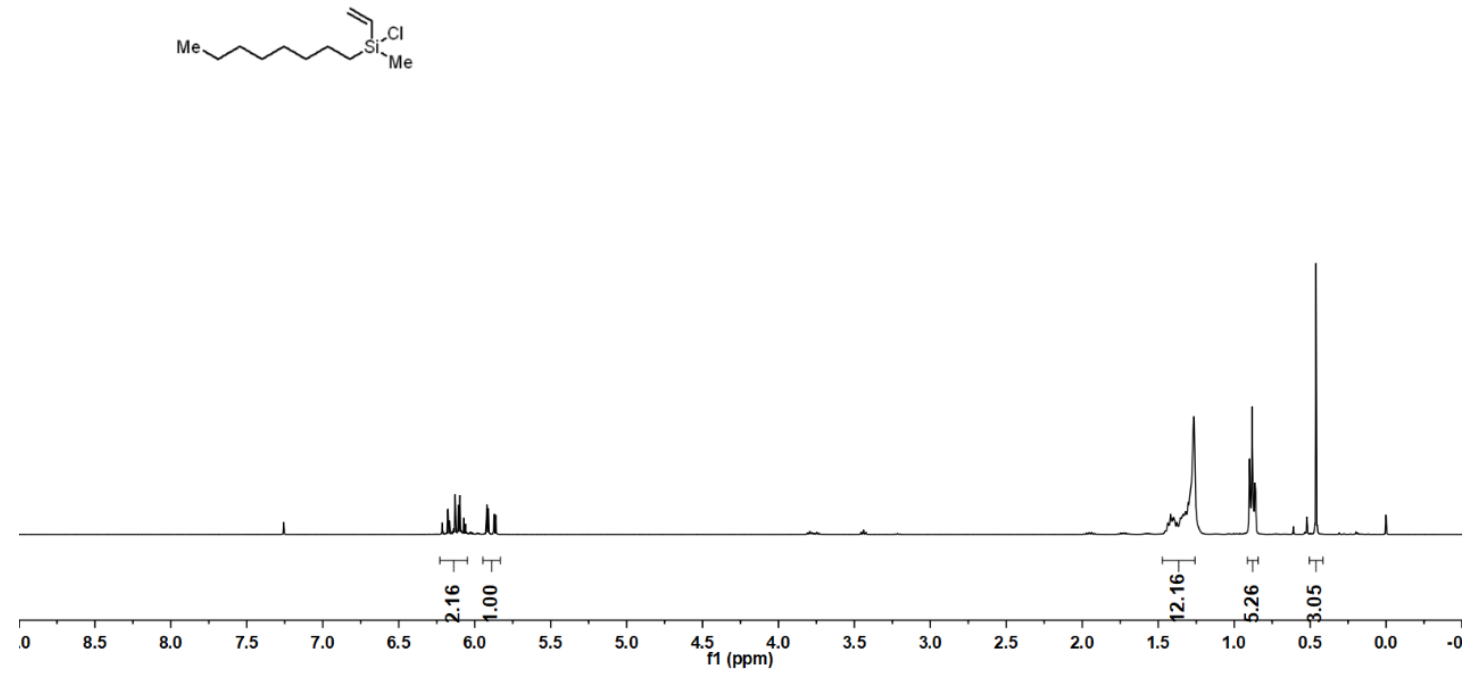

商芦
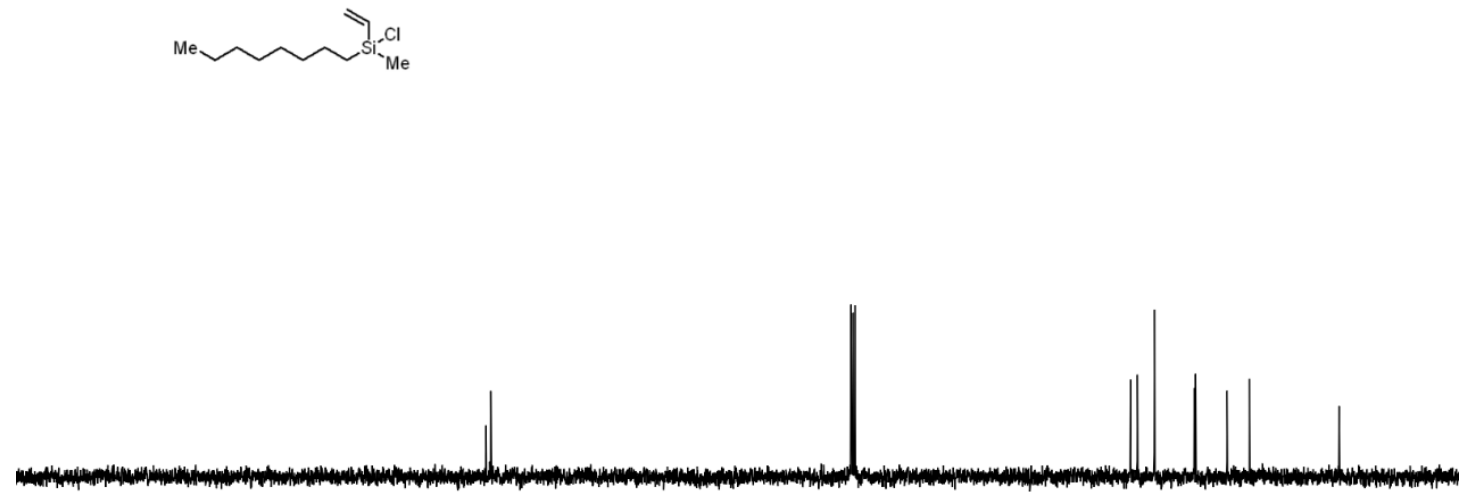

$\begin{array}{llllllllllll}10 & 200 & 190 & 180 & 170 & 160 & 150 & 140 & 130 & 120 & 110 & \underset{\mathrm{f} 1(\mathrm{ppm})}{90}\end{array}$ 
2h; $\left.{ }^{29} \mathrm{Si} \mathrm{NMR} \mathrm{(79} \mathrm{MHz,} \mathrm{CDCl}\right)$

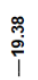

$\mathrm{Me}^{\mathrm{Sl}_{\mathrm{C}}^{\mathrm{Cl}} \mathrm{Me}}$

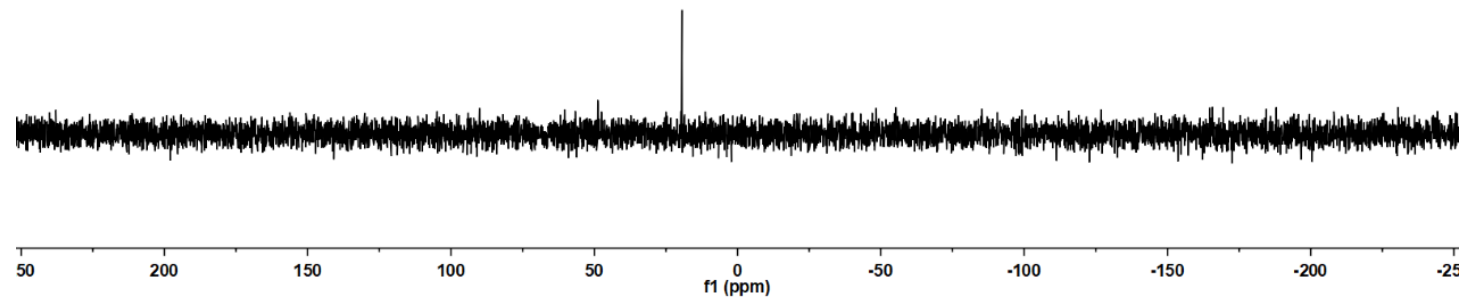


2j; ${ }^{1} \mathrm{H}$ NMR (400 MHz, CDCl 3$) ;{ }^{13} \mathrm{C}$ NMR (100 MHz, CDCl $)$

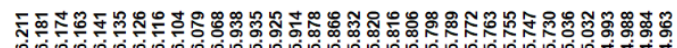

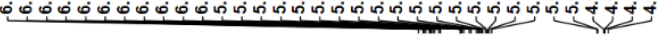

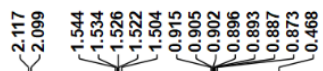

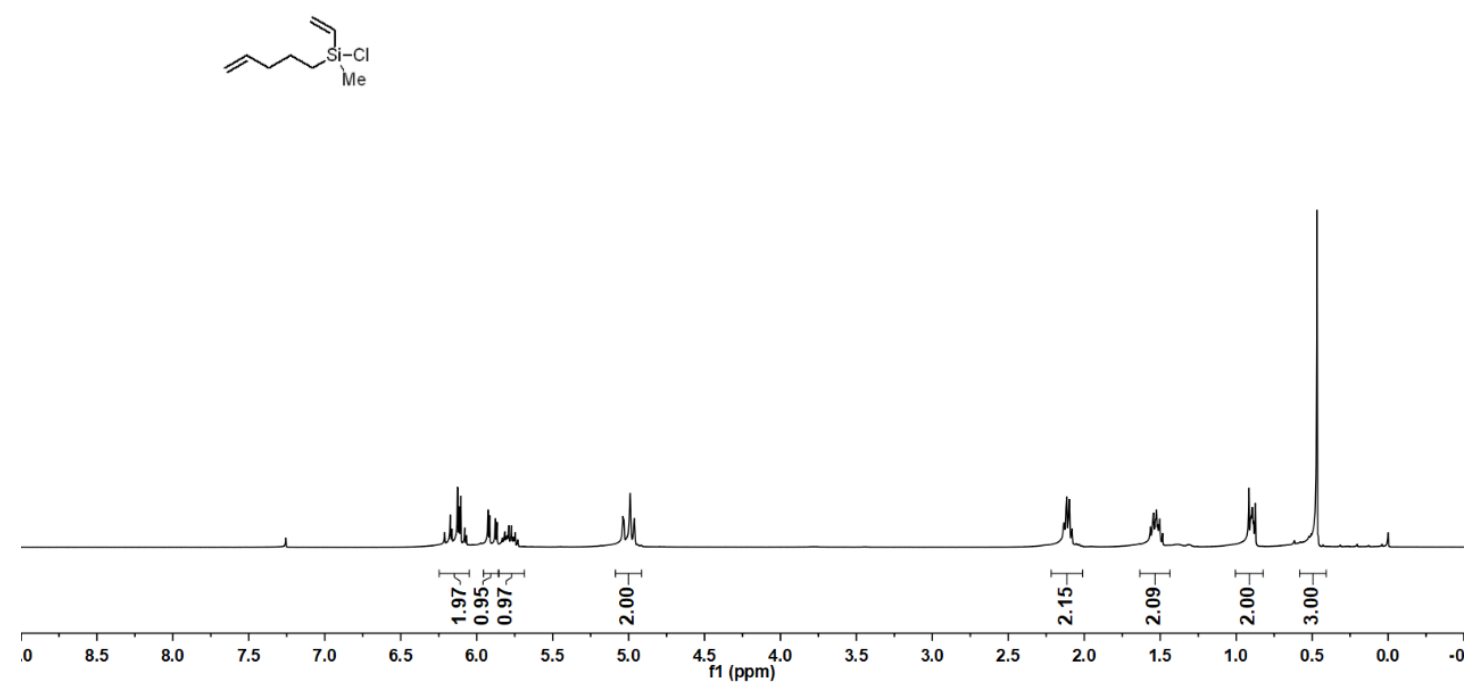

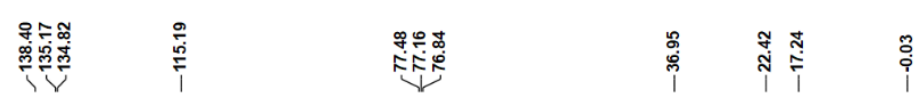

$\overbrace{\substack{\text { si-cl } \\ \text { Me }}}^{\text {s. }}$

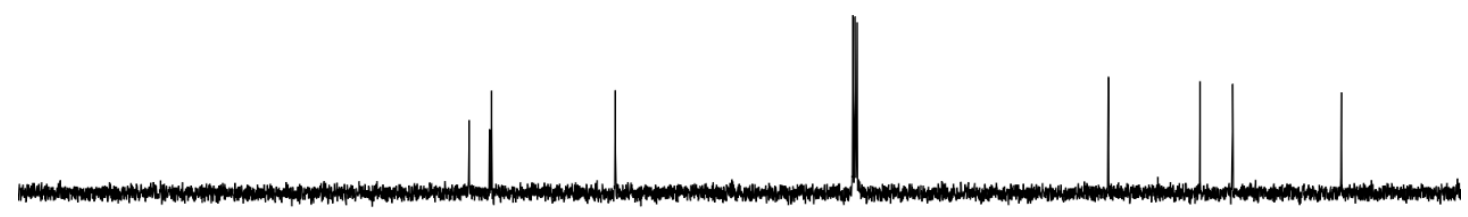

$\begin{array}{llllllllllll}10 & 200 & 190 & 180 & 170 & 160 & 150 & 140 & 130 & 120 & 110 & 100 \\ \mathrm{f} 1(\mathrm{ppm}) & 90\end{array}$ 
2j; $\left.{ }^{29} \mathrm{Si} \mathrm{NMR} \mathrm{(79} \mathrm{MHz,} \mathrm{CDCl}_{3}\right)$

윰

$\overbrace{\substack{\text { si-cl } \\ \text { ime }}}^{\text {s. }}$

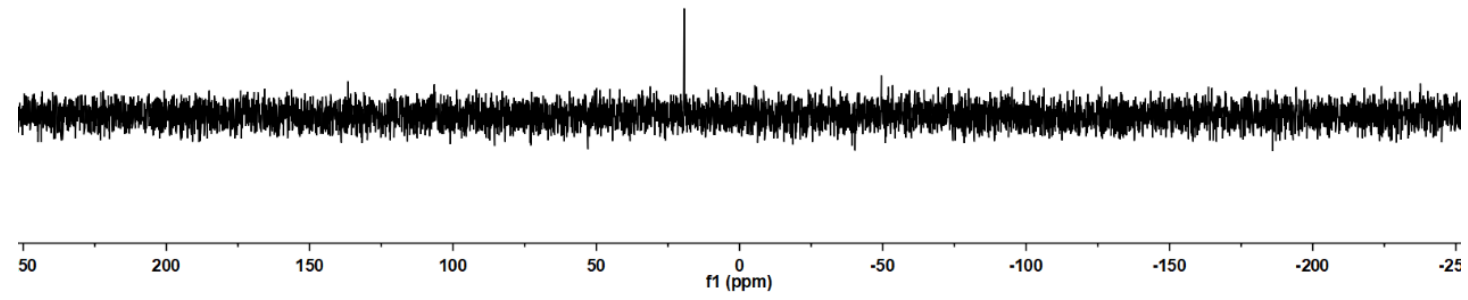


3a; ${ }^{1} \mathrm{H}$ NMR (400 MHz, CDCl $\left.) ;{ }^{13} \mathrm{C} \mathrm{NMR} \mathrm{(100} \mathrm{MHz}, \mathrm{CDCl}_{3}\right)$

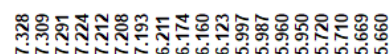

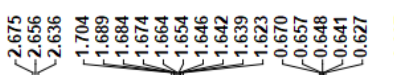

S. Me, me

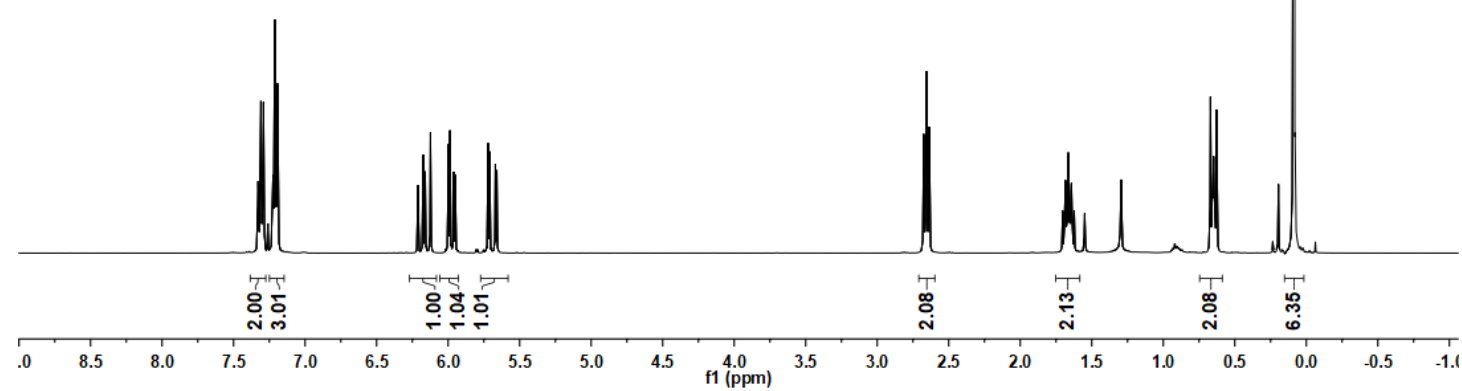

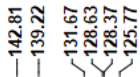

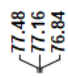

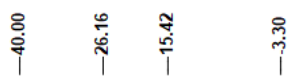

On wister

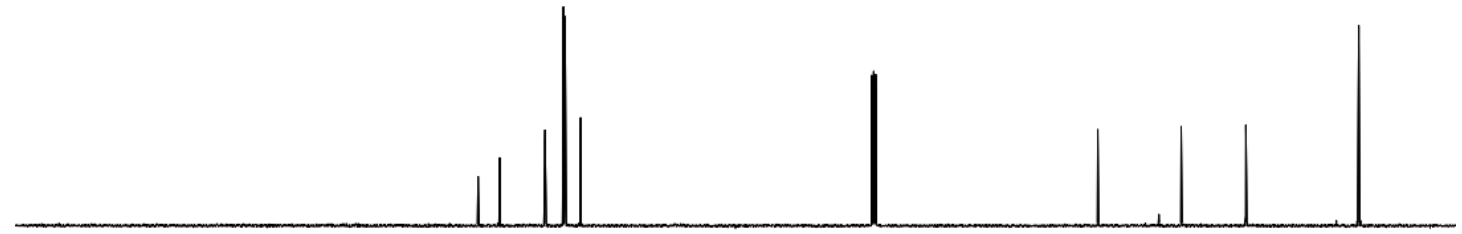

$\begin{array}{llllllllllllllllllllllllllll}210 & 200 & 190 & 180 & 170 & 160 & 150 & 140 & 130 & 120 & 110 & 100 & 90 & 80 & 70 & 60 & 50 & 40 & 30 & 20 & 10 & 0 & -10\end{array}$ 
3a; ${ }^{29} \mathrm{Si}$ NMR (79 MHz, CDCl 3$)$

$\frac{9}{i}$

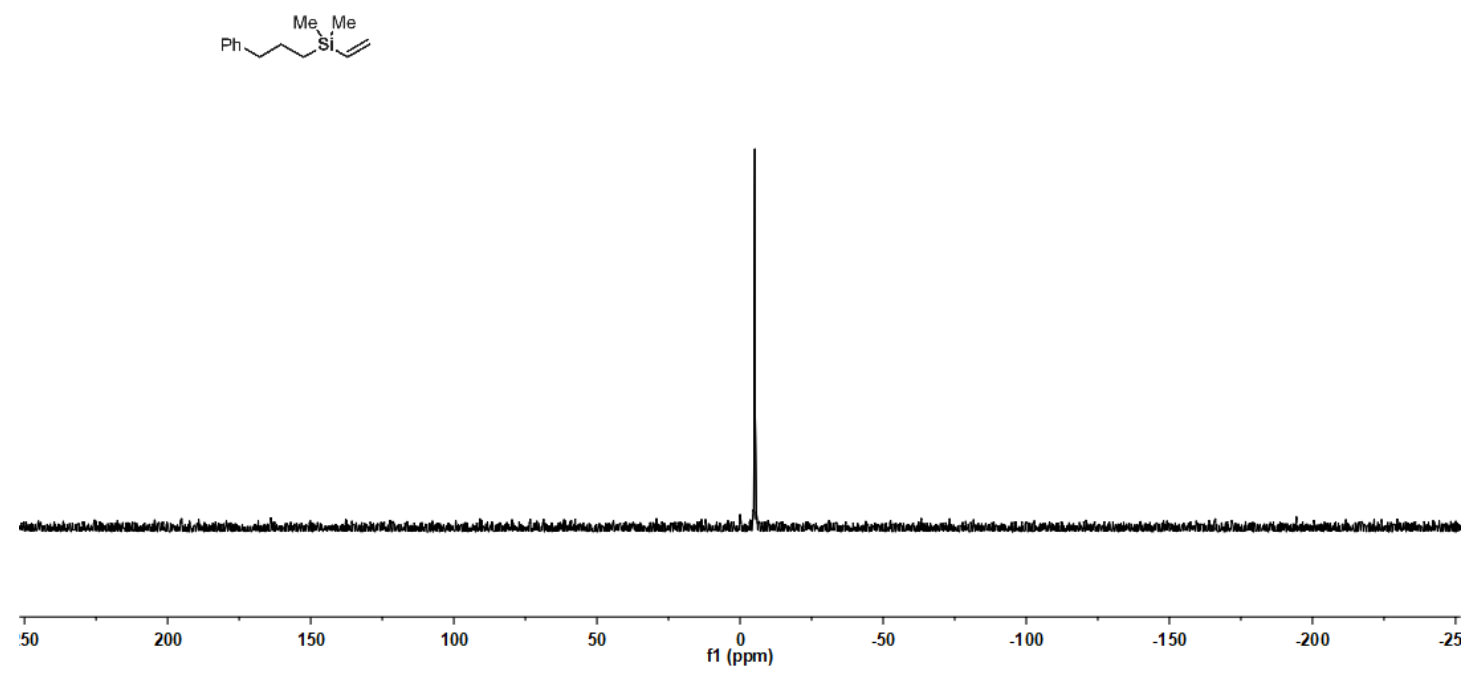


3b; ${ }^{1} \mathrm{H}$ NMR (400 MHz, CDCl$) ;{ }^{13} \mathrm{C}$ NMR (100 MHz, CDCl$)$

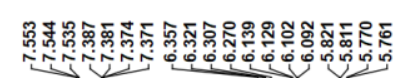

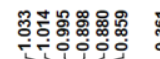

Me

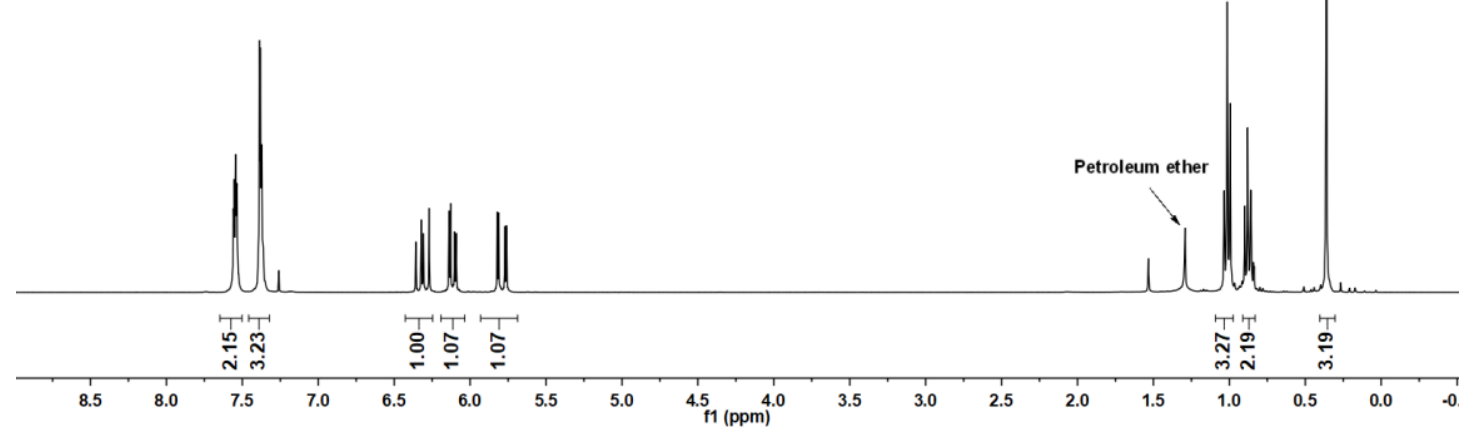

ธㅉำ 뜽ำ

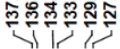

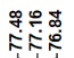

里过
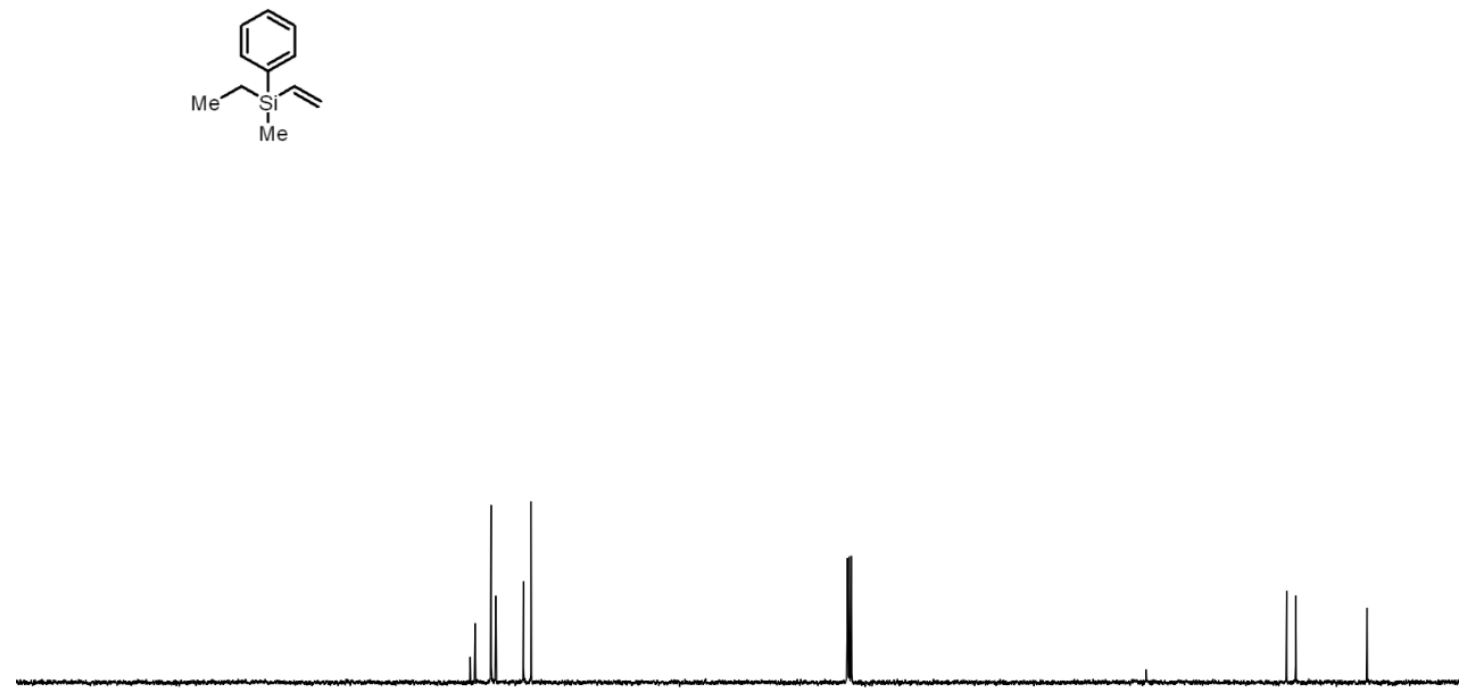

$\begin{array}{lllllllllllllllllllllllllllllllllllll}10 & 200 & 190 & 180 & 170 & 160 & 150 & 140 & 130 & 120 & 110 & 100 & 90 & 80 & 70 & 60 & 50 & 40 & 30 & 20 & 10 & 0 & -10 & -2\end{array}$ 
3b; ${ }^{29} \mathrm{Si}$ NMR (79 MHz, CDCl$)$

:

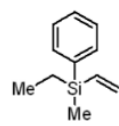

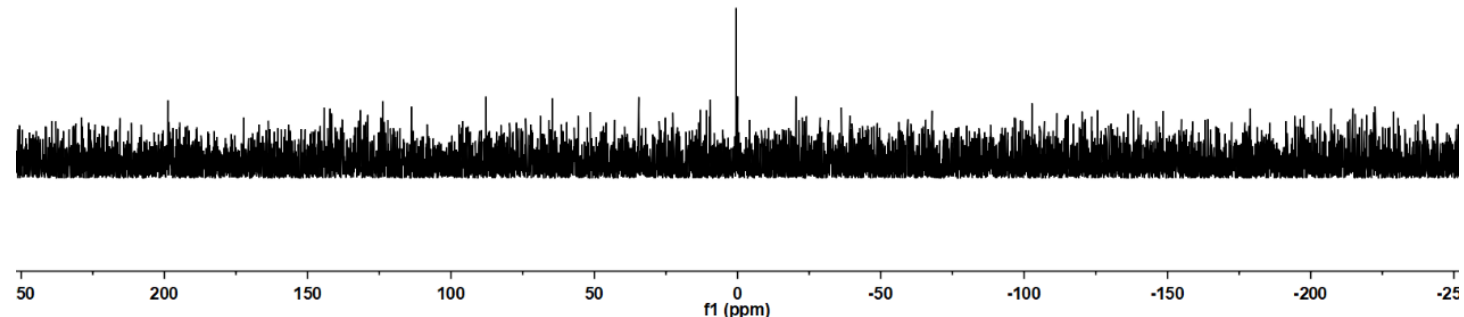


3c; ${ }^{1} \mathrm{H}$ NMR (400 MHz, CDCl 3$\left.) ;{ }^{13} \mathrm{C} \mathrm{NMR} \mathrm{(100} \mathrm{MHz}, \mathrm{CDCl}_{3}\right)$

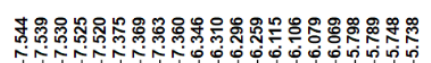

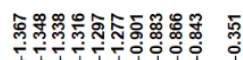
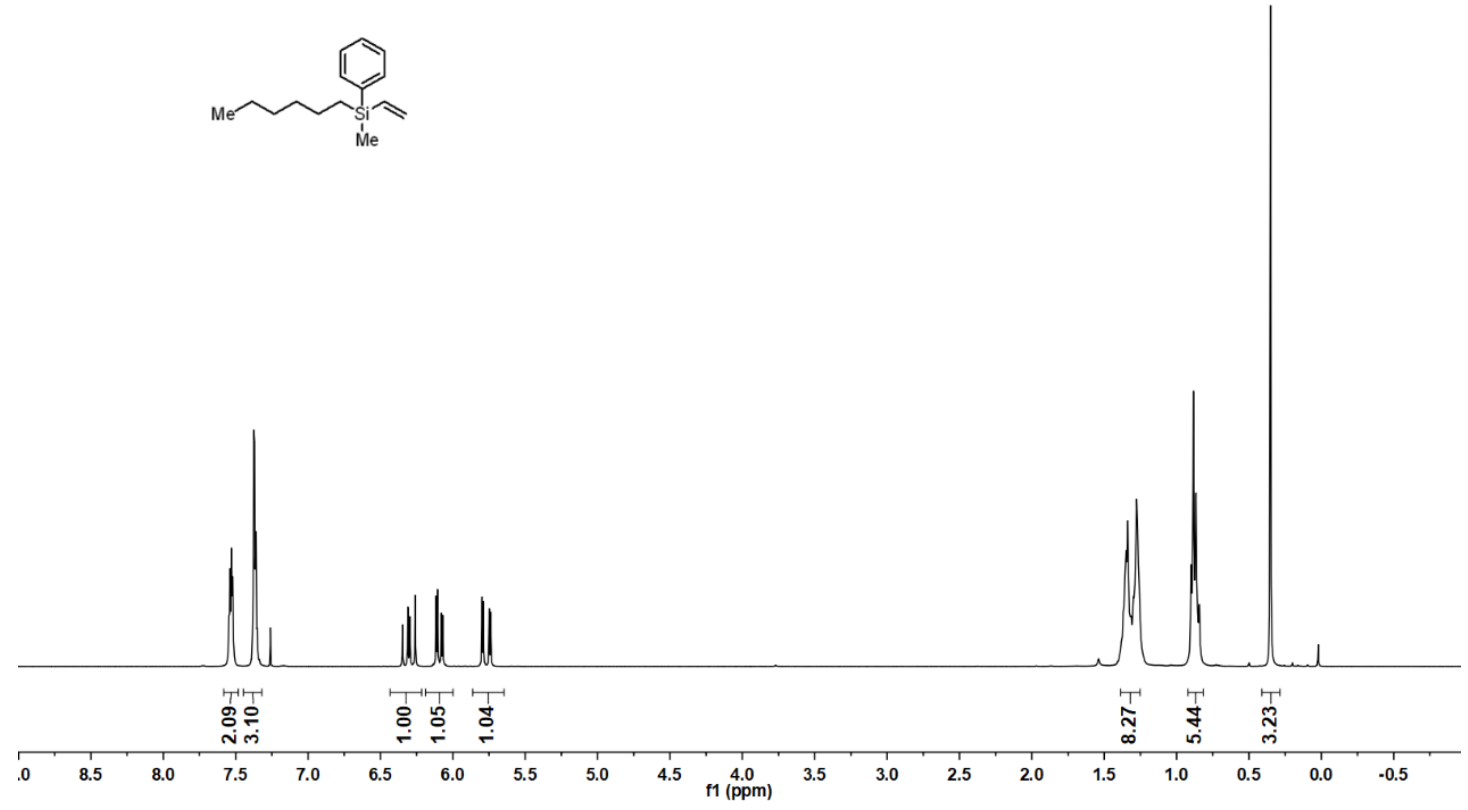

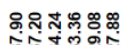

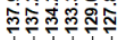

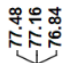

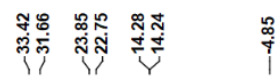
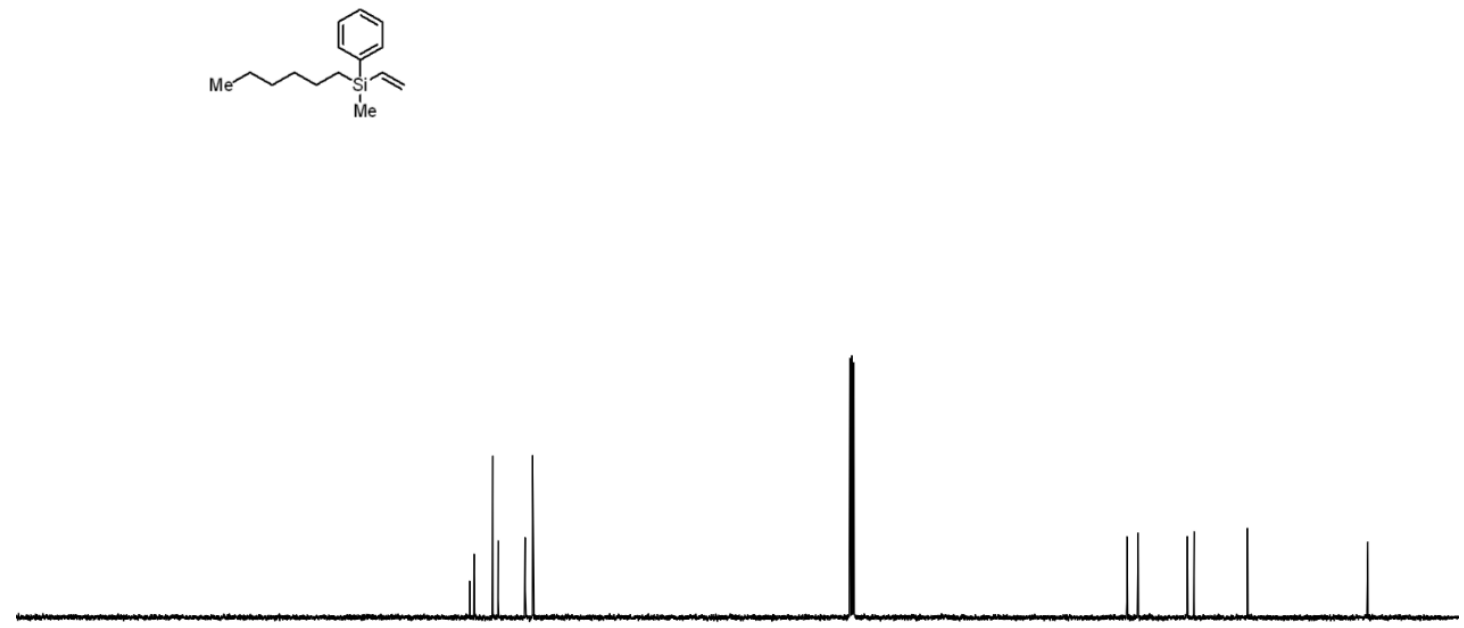

\begin{tabular}{lllllllllllll}
\hline 10 & 200 & 190 & 180 & 170 & 160 & 150 & 140 & 130 & 120 & 110 & $\underset{100}{10} 90$ \\
$f 1(\mathrm{ppm})$
\end{tabular}

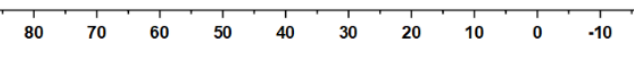


3c; ${ }^{29} \mathrm{Si}$ NMR (79 MHz, CDCl 3 )

¿্ট<smiles>CCCCCC1(C)C=CC1</smiles>

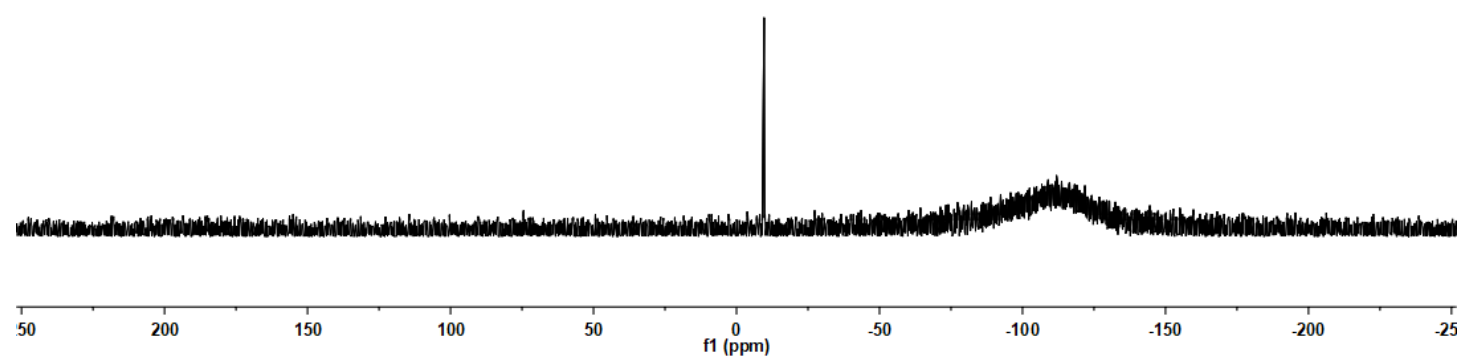


3d; ${ }^{1} \mathrm{H}$ NMR (400 MHz, $\left.\mathrm{CDCl}_{3}\right) ;{ }^{13} \mathrm{C} \mathrm{NMR}\left(100 \mathrm{MHz}, \mathrm{CDCl}_{3}\right)$

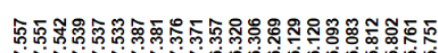

管

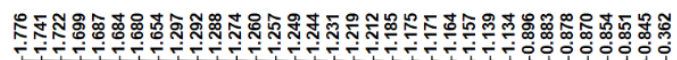

(1)

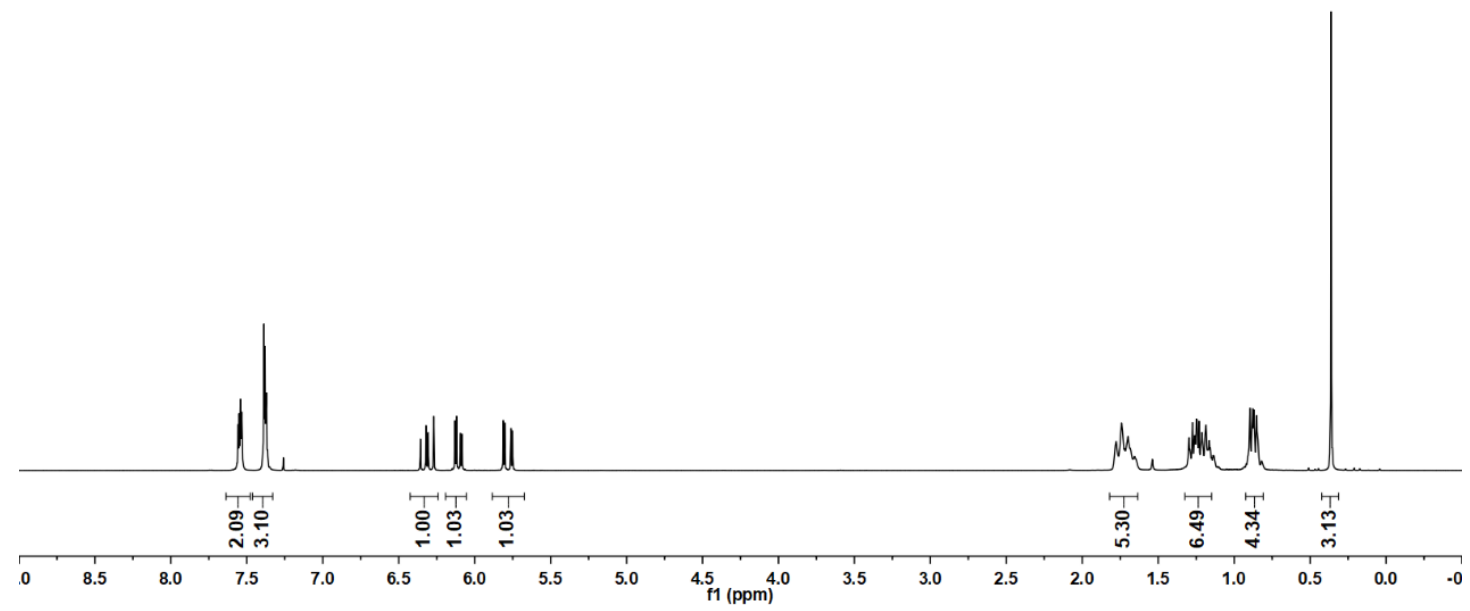

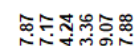

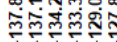

通

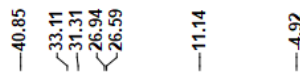
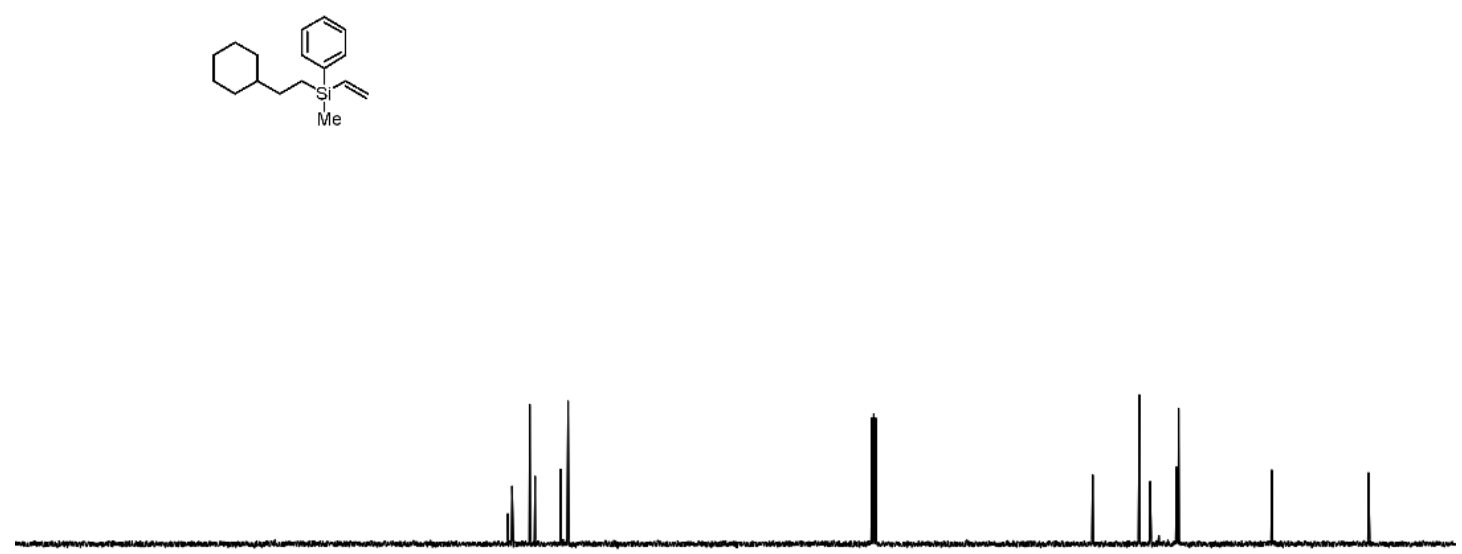

$\begin{array}{llllllllllllllllllllllllll}210 & 200 & 190 & 180 & 170 & 160 & 150 & 140 & 130 & 120 & 110 & \begin{array}{c}100 \\ \mathrm{f} 1\end{array}(\mathrm{ppm}) & 90 & 80 & 70 & 60 & 50 & 40 & 30 & 20 & 10 & 0 & -10\end{array}$ 
3d; ${ }^{29} \mathrm{Si}$ NMR (79 MHz, CDCl$)$

$\frac{n}{9}$

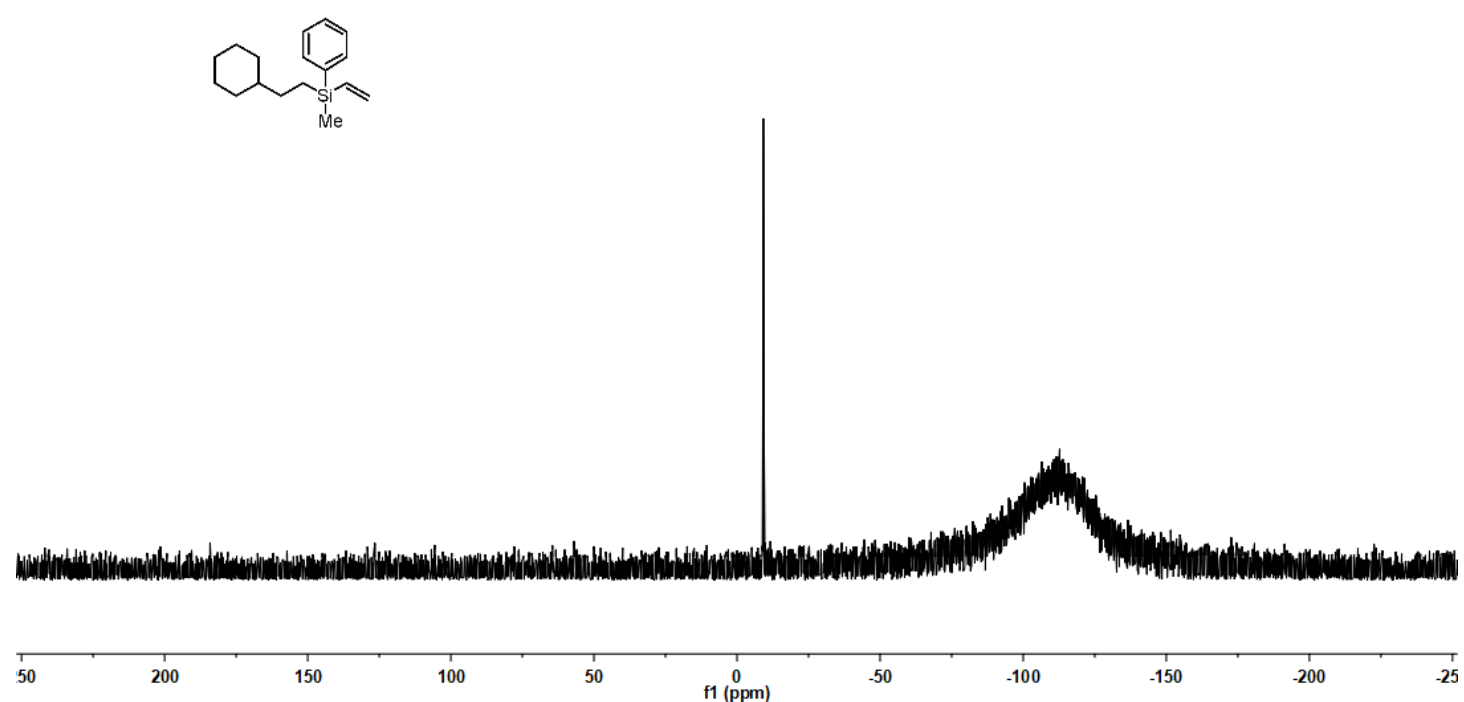


3e; ${ }^{1} \mathrm{H}$ NMR (400 MHz, CDCl 3$) ;{ }^{13} \mathrm{C}$ NMR (100 MHz, CDCl $)$

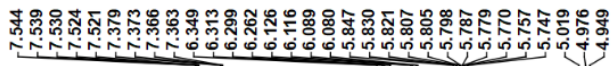

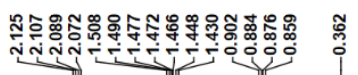<smiles></smiles>

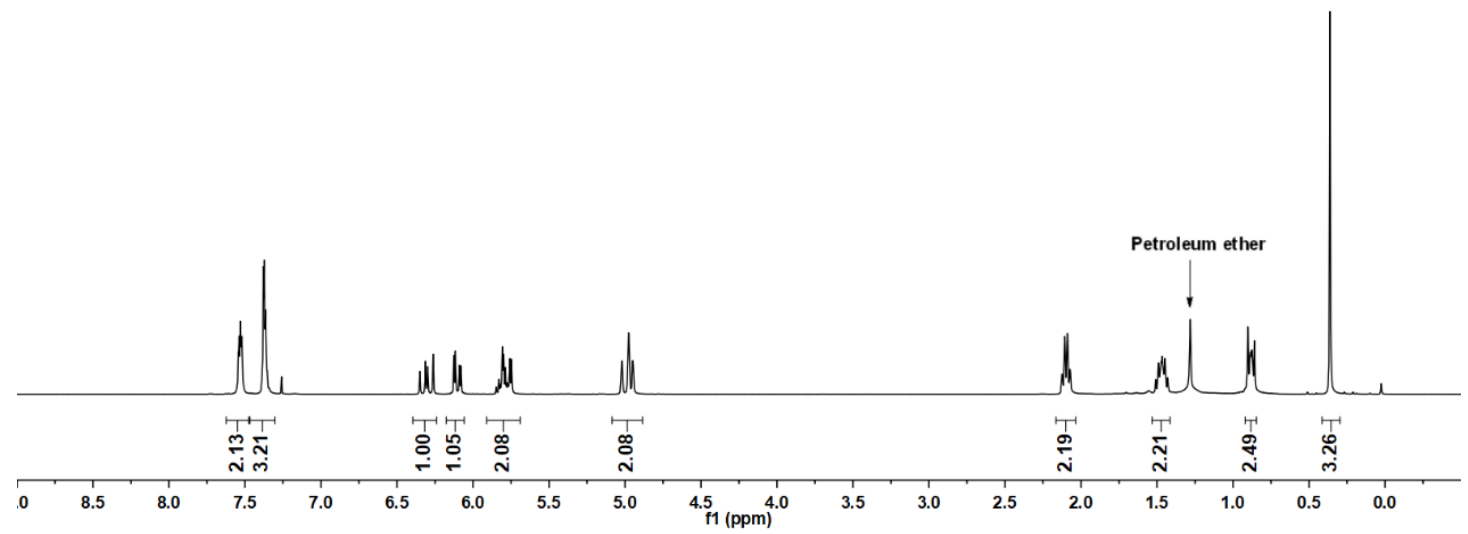

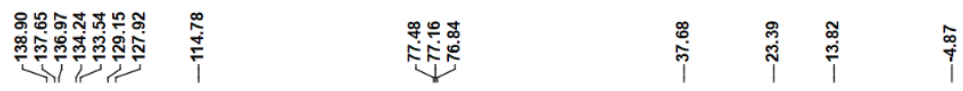
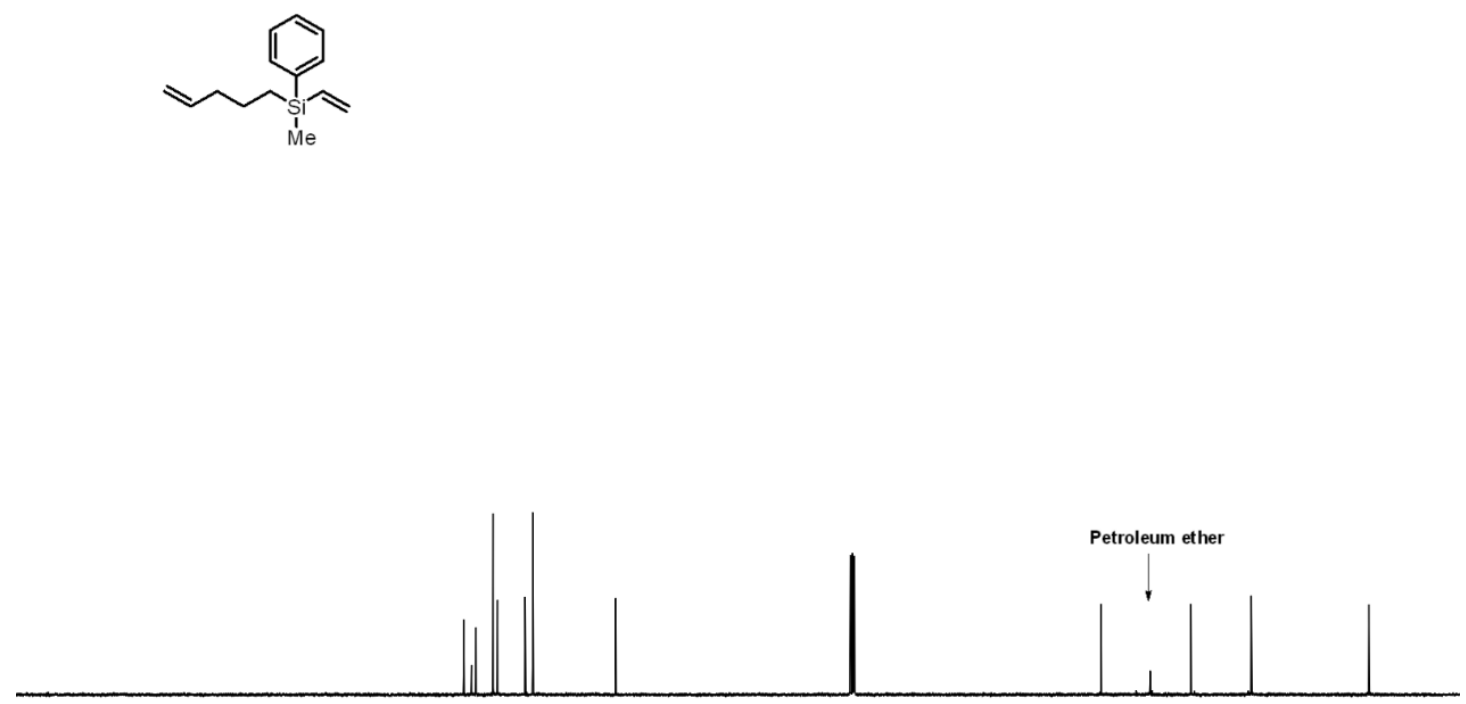

\begin{tabular}{|llllllllllllllllllllllllllllllllllll}
\hline 10 & 200 & 190 & 180 & 170 & 160 & 150 & 140 & 130 & 120 & 110 & 100 & 90 & 80 & 70 & 60 & 50 & 40 & 30 & 20 & 10 & 0 & -10
\end{tabular} 
3e; ${ }^{29} \mathrm{Si}$ NMR (79 MHz, CDCl 3$)$

$\stackrel{\circ}{\circ}$
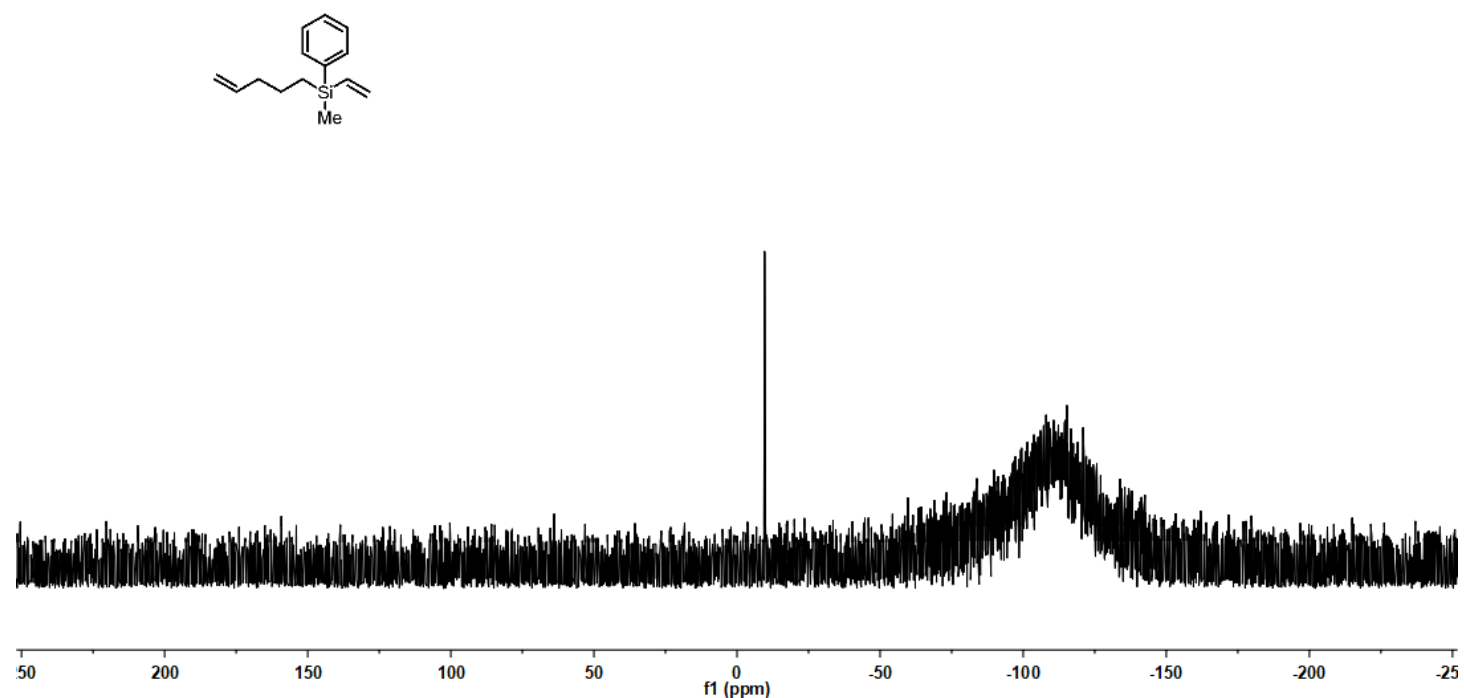
3f; ${ }^{1} \mathrm{H}$ NMR (400 MHz, $\left.\mathrm{CDCl}_{3}\right) ;{ }^{13} \mathrm{C}$ NMR (100 $\left.\mathrm{MHz}, \mathrm{CDCl}_{3}\right)$

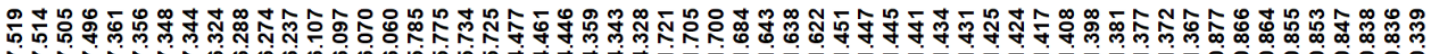

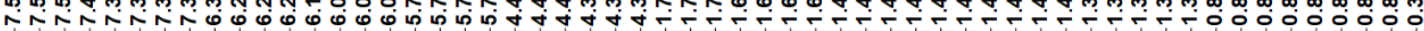
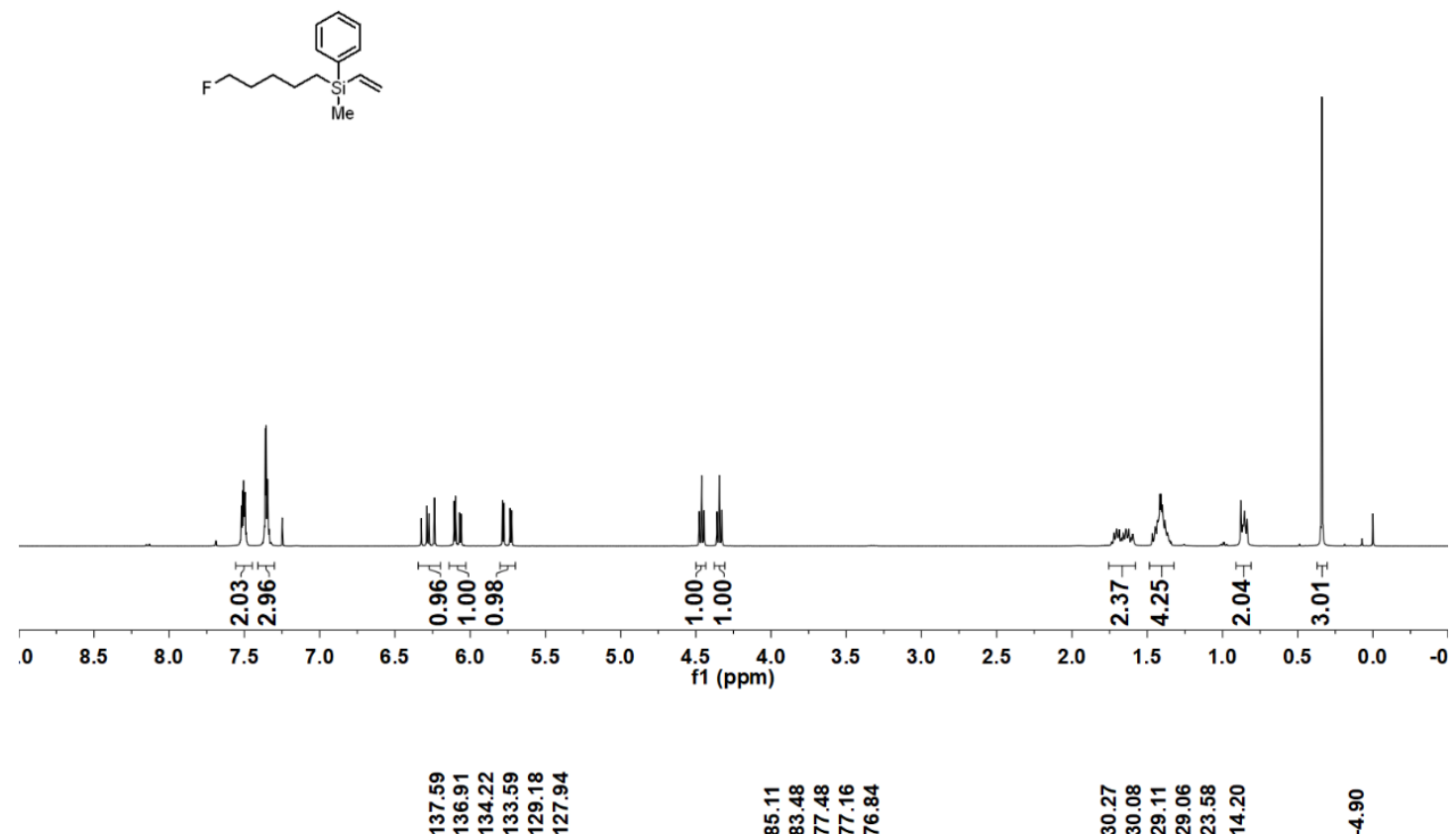

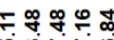

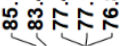

กุ गิ่

$\stackrel{\circ}{\text { ஒ }}$
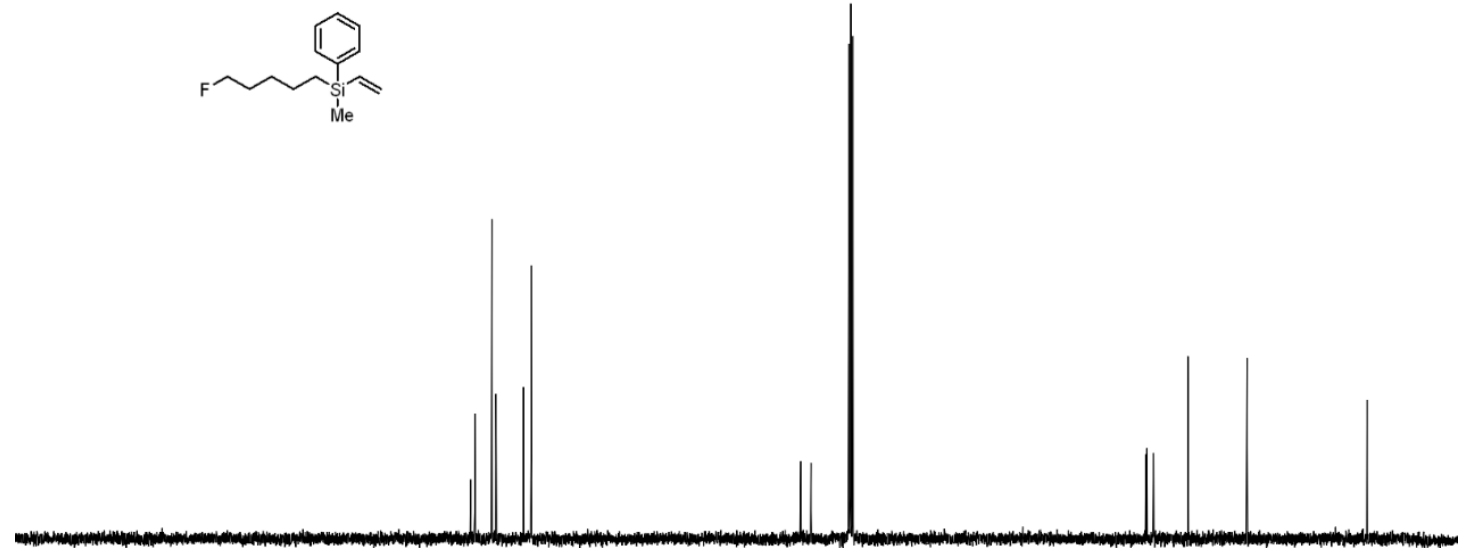

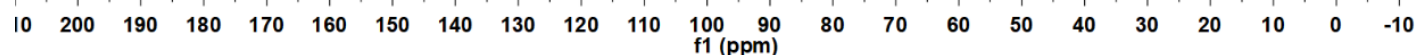


3f; ${ }^{19}$ F NMR (376 MHz, CDCl $)$ ); ${ }^{29} \mathrm{Si}$ NMR (79 MHz, CDCl $)$

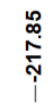

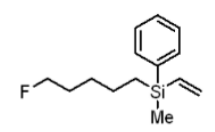

00

150

100

50

-100
$f 1(\mathrm{ppm})$

$-4 C$

$\stackrel{8}{i}$
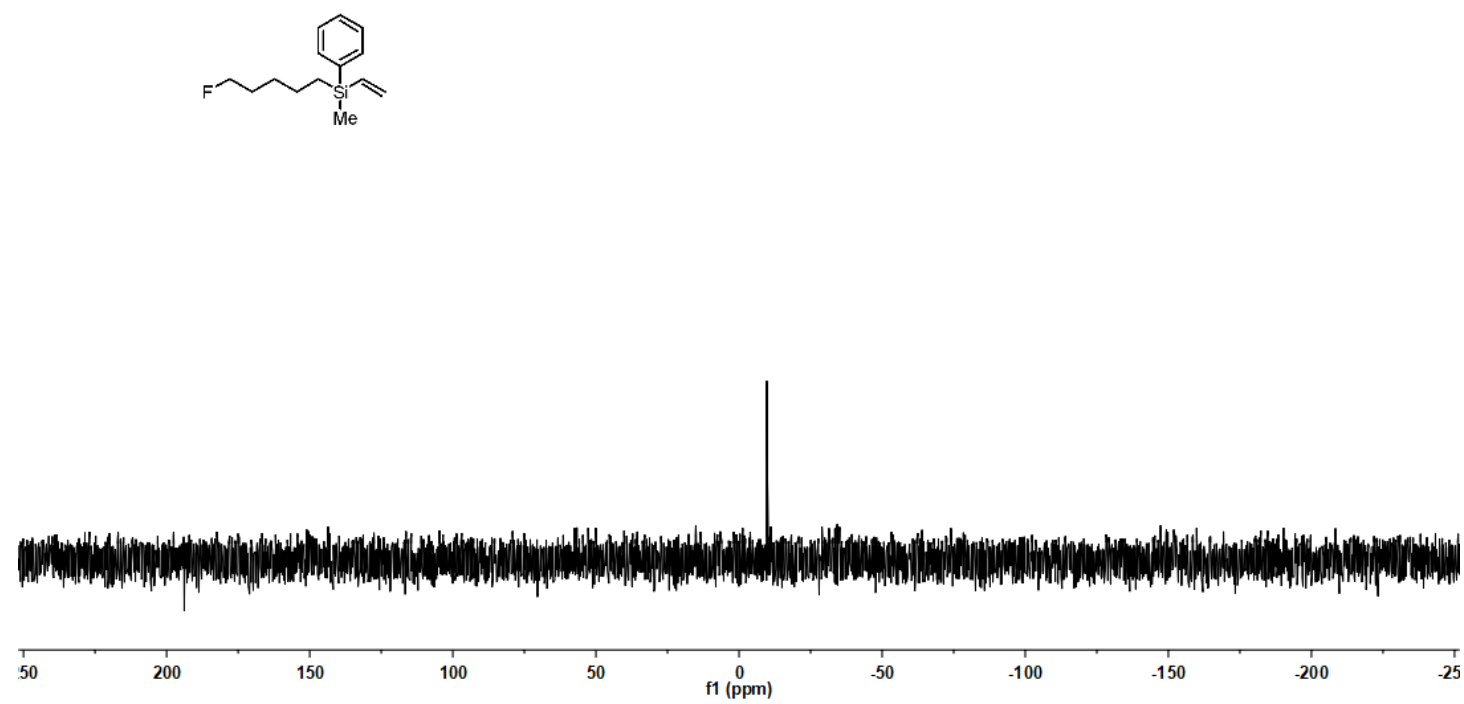

S44 
3g; ${ }^{1} \mathrm{H}$ NMR (400 MHz, CDCl $\left.) ;{ }^{13} \mathrm{C} \mathrm{NMR} \mathrm{(100} \mathrm{MHz}, \mathrm{CDCl}_{3}\right)$

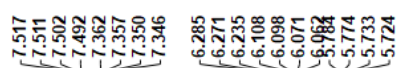

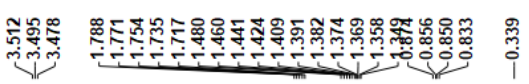
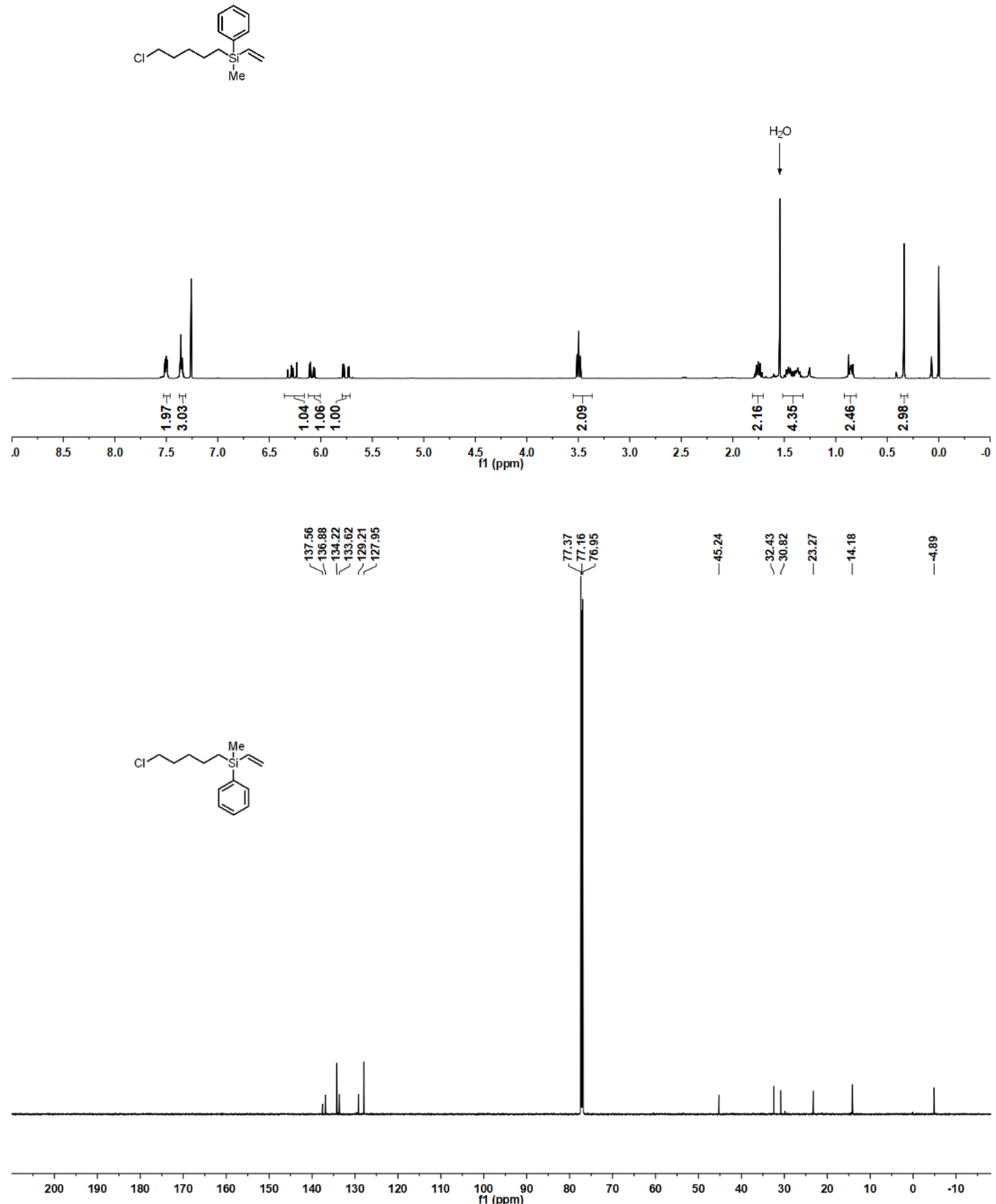
3g; ${ }^{29} \mathrm{Si}$ NMR (79 MHz, CDCl 3$)$

$\stackrel{?}{i}$

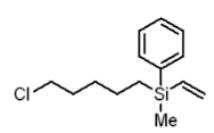

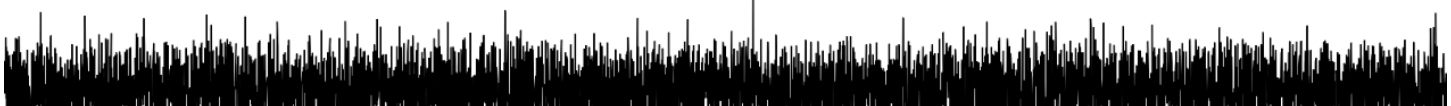

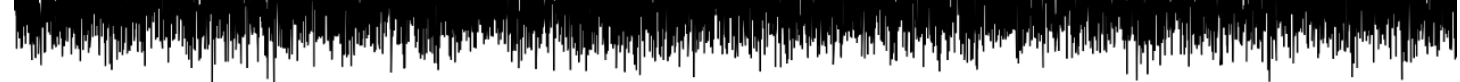

\begin{tabular}{|c|c|c|c|c|c|c|c|c|c|}
\hline 50 & 200 & 150 & 100 & 50 & $\begin{array}{c}0 \\
\mathrm{f} 1(\mathrm{ppm})\end{array}$ & -50 & -100 & -150 & -200 \\
\hline
\end{tabular}


3h; ${ }^{1} \mathrm{H}$ NMR (400 MHz, CDCl$) ;{ }^{13} \mathrm{C}$ NMR (100 MHz, CDCl 3$)$

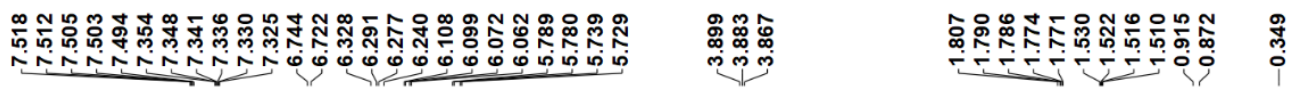
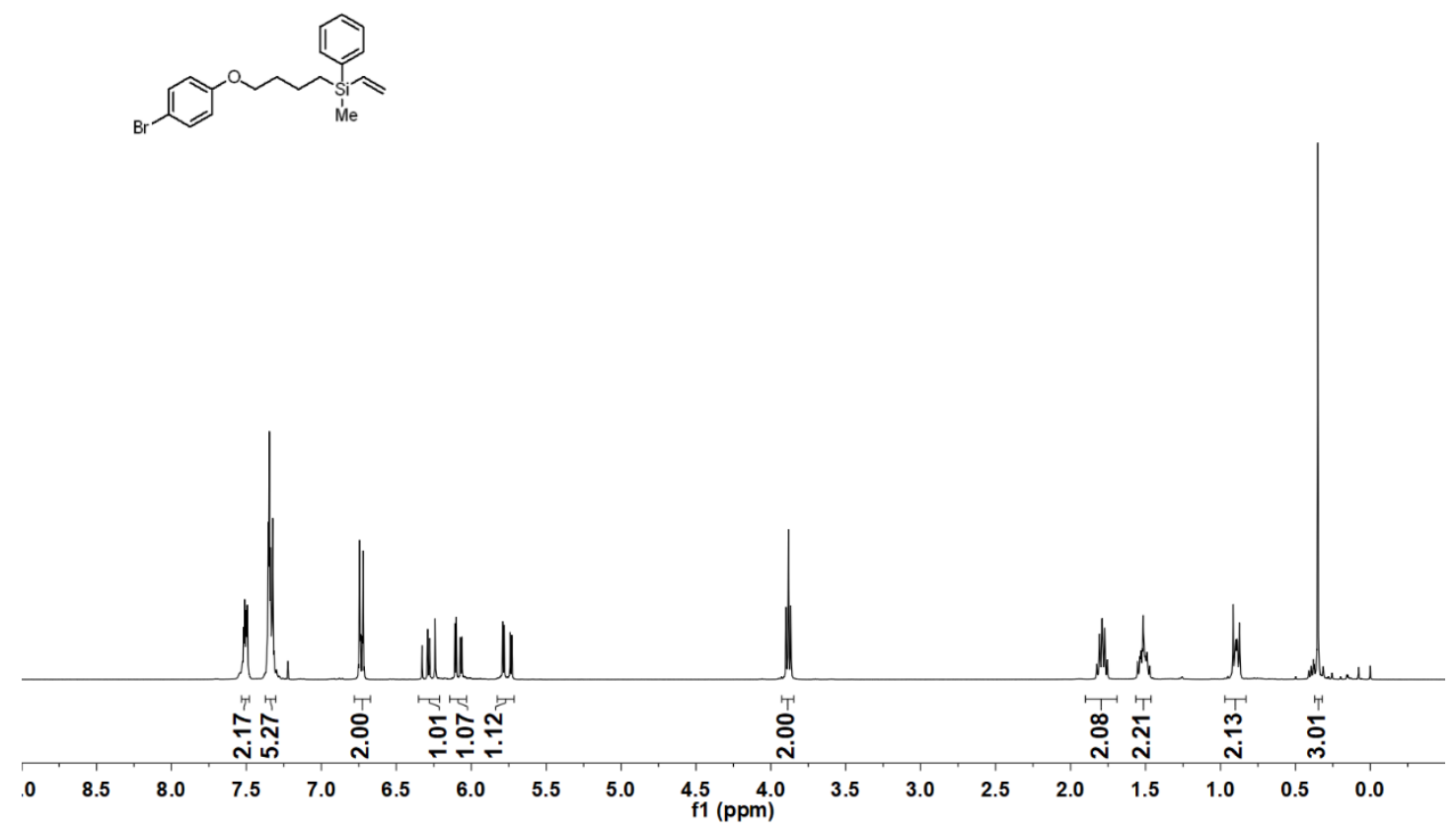

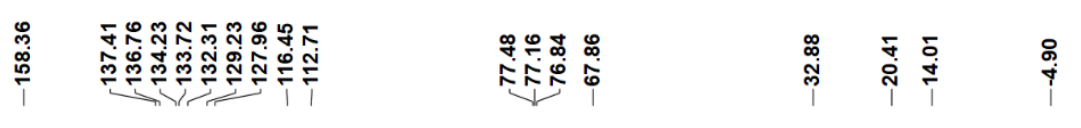

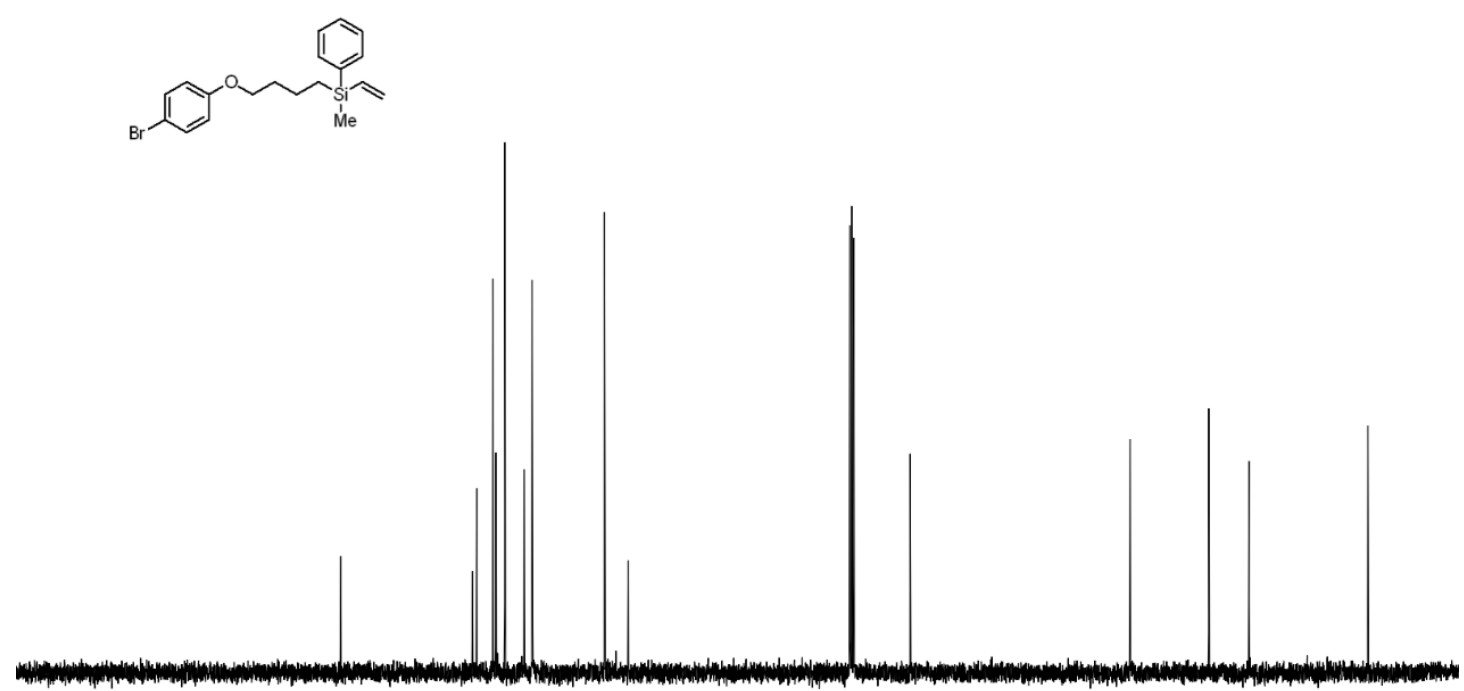

$\begin{array}{llllllllllllllllllllll}10 & 200 & 190 & 180 & 170 & 160 & 150 & 140 & 130 & 120 & 110 & \begin{array}{l}100 \\ \mathrm{f} 1(\mathrm{ppm})\end{array} & 80 & 70 & 60 & 50 & 40 & 30 & 20 & 10 & 0 & -10\end{array}$ 
3h; ${ }^{29} \mathrm{Si}$ NMR (79 MHz, CDCl$)$

$\stackrel{\overline{0}}{i}$

(

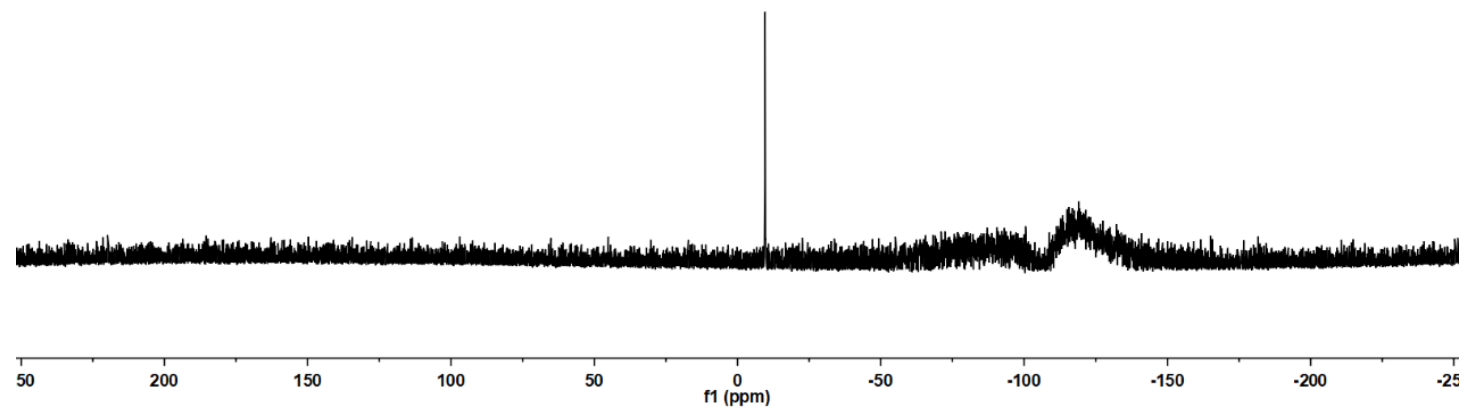


3i; ${ }^{1} \mathrm{H}$ NMR (400 MHz, CDCl $)$; ${ }^{13} \mathrm{C}$ NMR (100 MHz, CDCl $)$

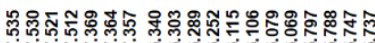

तritit ou

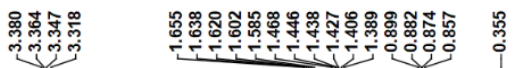
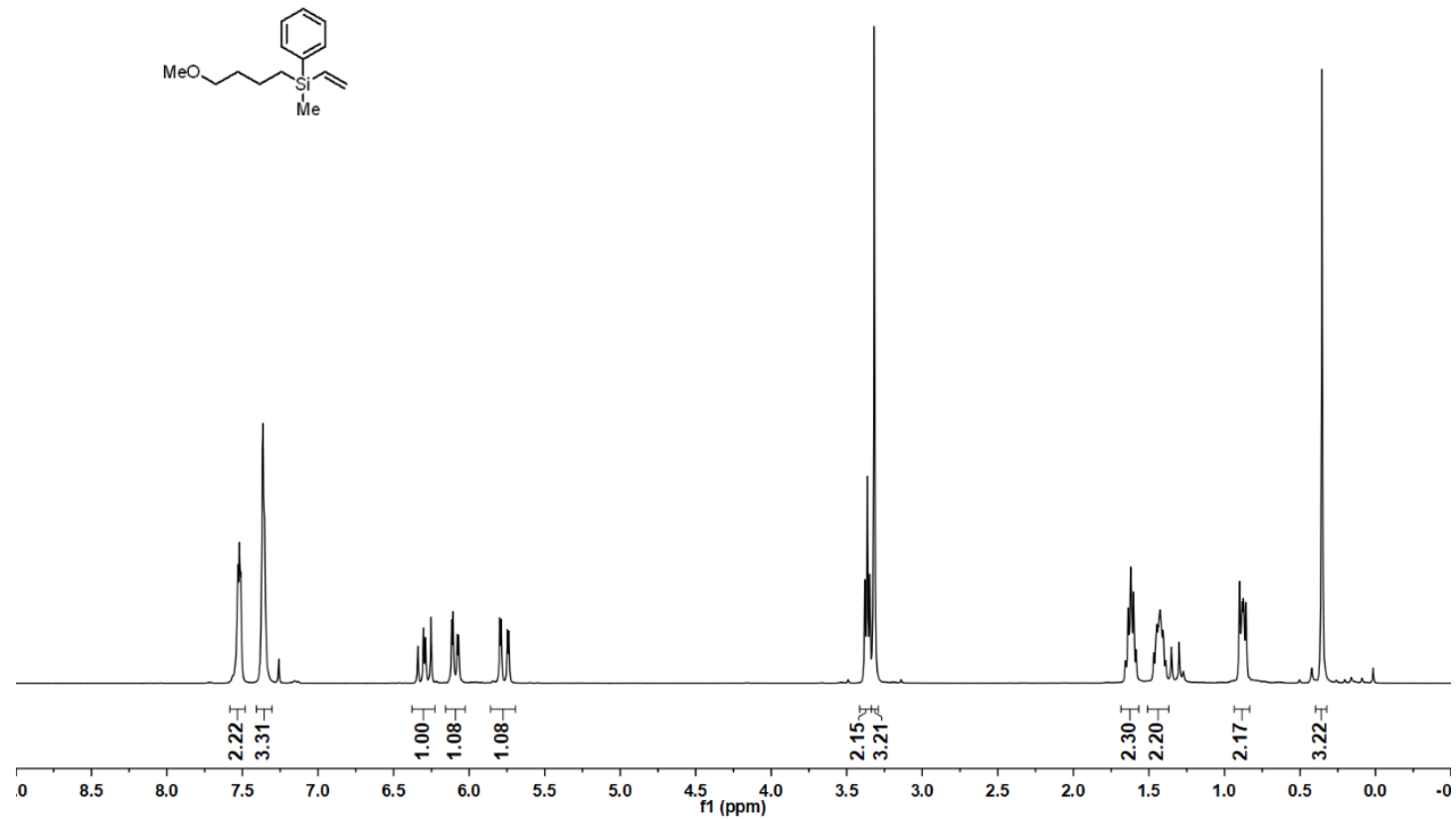

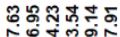

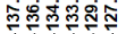

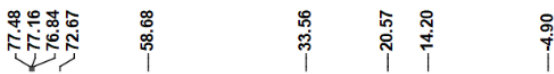
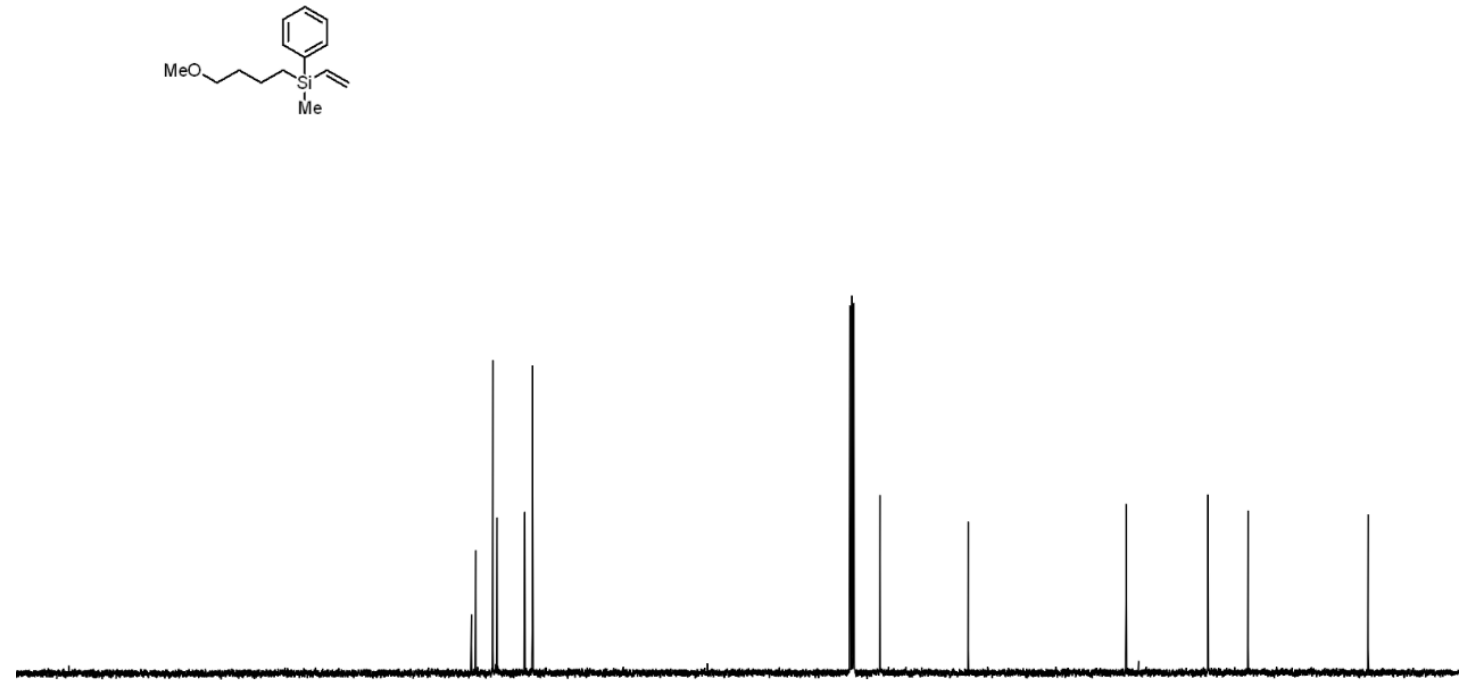

$\begin{array}{llllllllllll}10 & 200 & 190 & 180 & 170 & 160 & 150 & 140 & 130 & 120 & 110 & 100 \\ 11(\mathrm{ppm}) & 90\end{array}$ 
3i; ${ }^{29} \mathrm{Si}$ NMR (79 MHz, $\left.\mathrm{CDCl}_{3}\right)$

\section{ఫ্ণ}
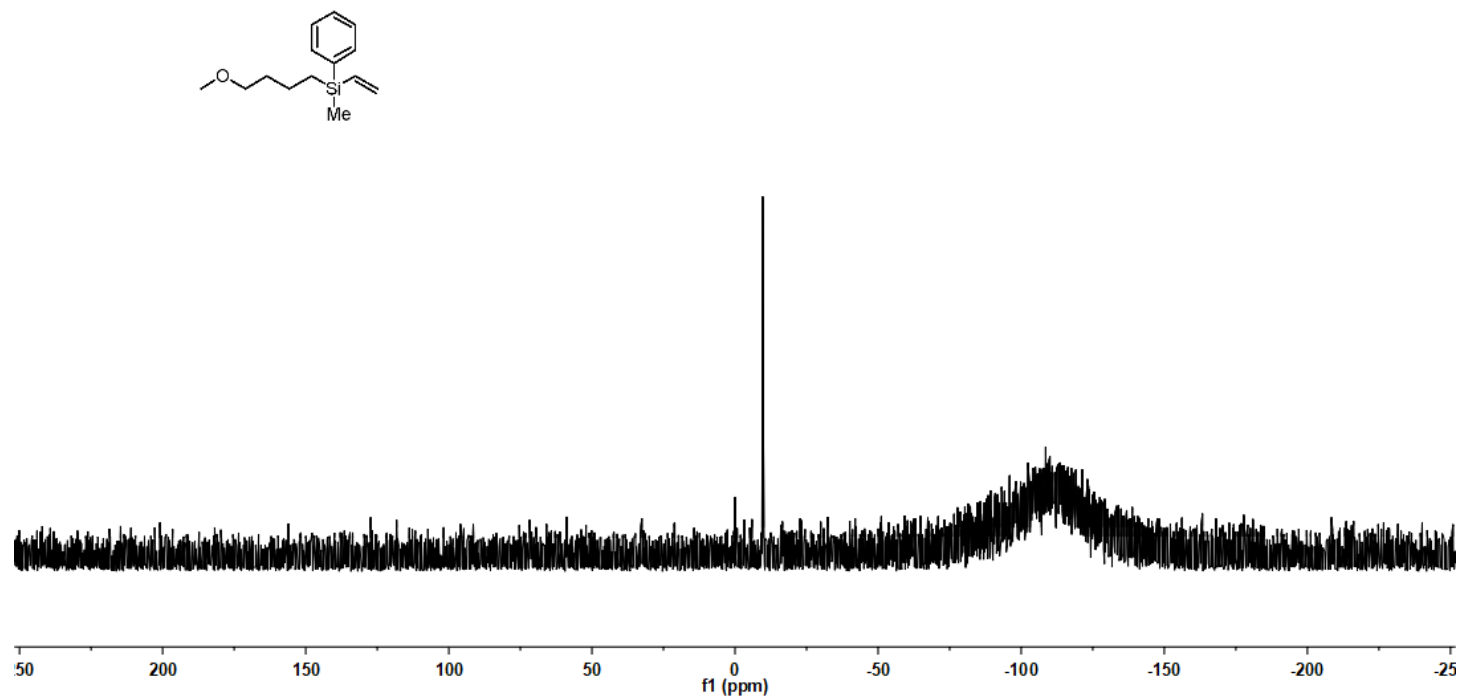
3j; ${ }^{1} \mathrm{H}$ NMR (400 MHz, CDCl 3$) ;{ }^{13} \mathrm{C}$ NMR (100 MHz, CDCl $)$

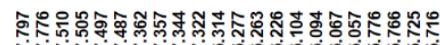

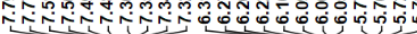

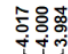

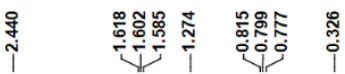

Tson

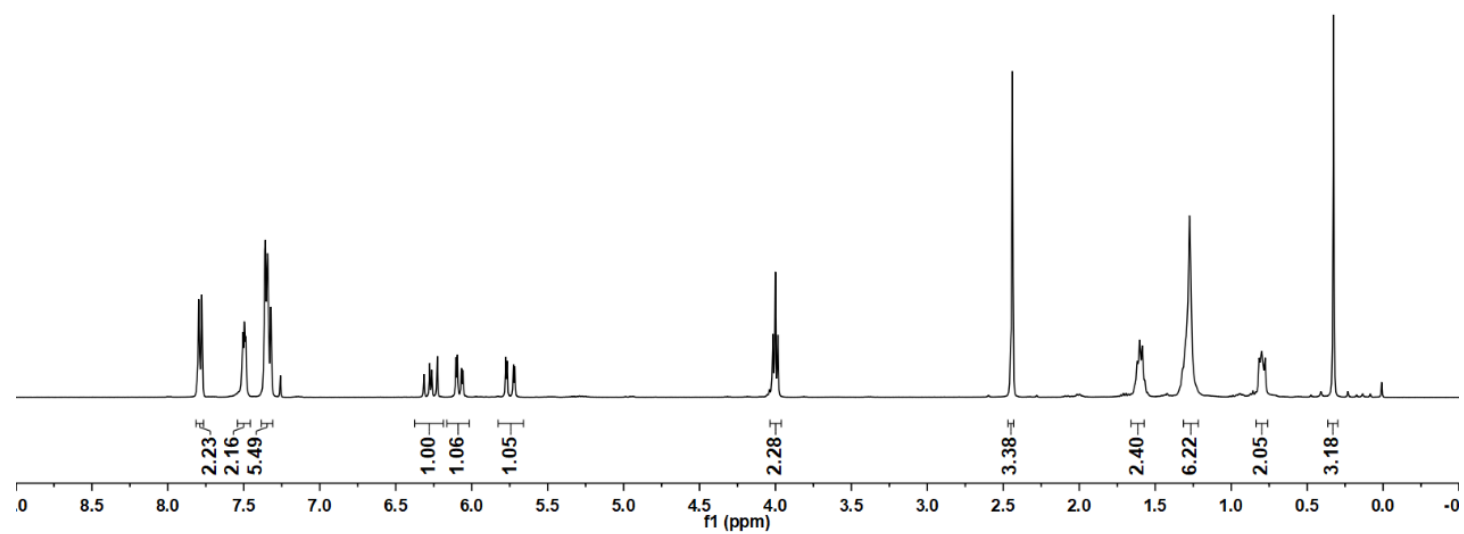

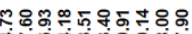

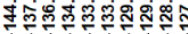

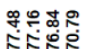

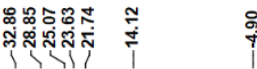
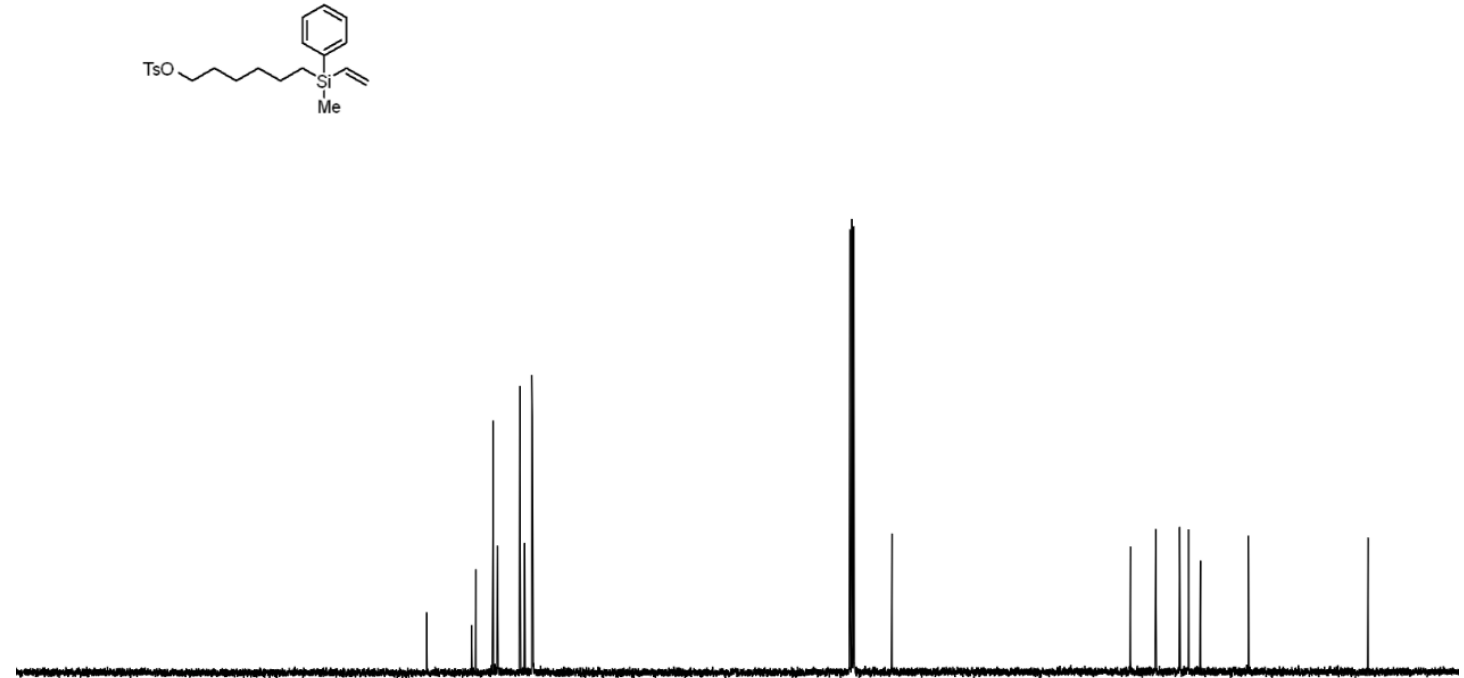

$\begin{array}{llllllllllll}10 & 200 & 190 & 180 & 170 & 160 & 150 & 140 & 130 & 120 & 110 & 100 \\ \mathrm{f} 1(\mathrm{ppm})\end{array}$ 
3j; ${ }^{29} \mathrm{Si}$ NMR (79 MHz, CDCl$)$

$\stackrel{\infty}{\circ}$
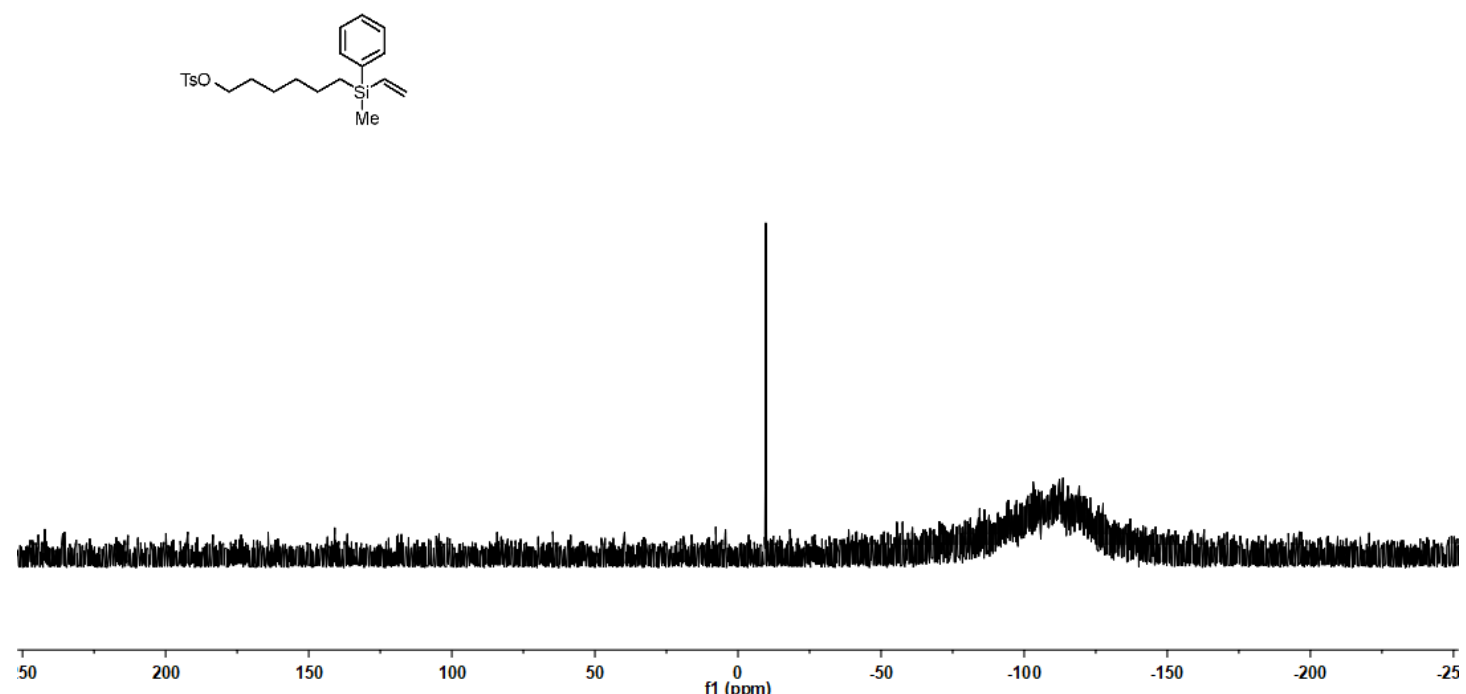
3k; ${ }^{1} \mathrm{H}$ NMR (400 MHz, $\left.\mathrm{CDCl}_{3}\right) ;{ }^{13} \mathrm{C} \mathrm{NMR}\left(100 \mathrm{MHz}, \mathrm{CDCl}_{3}\right)$
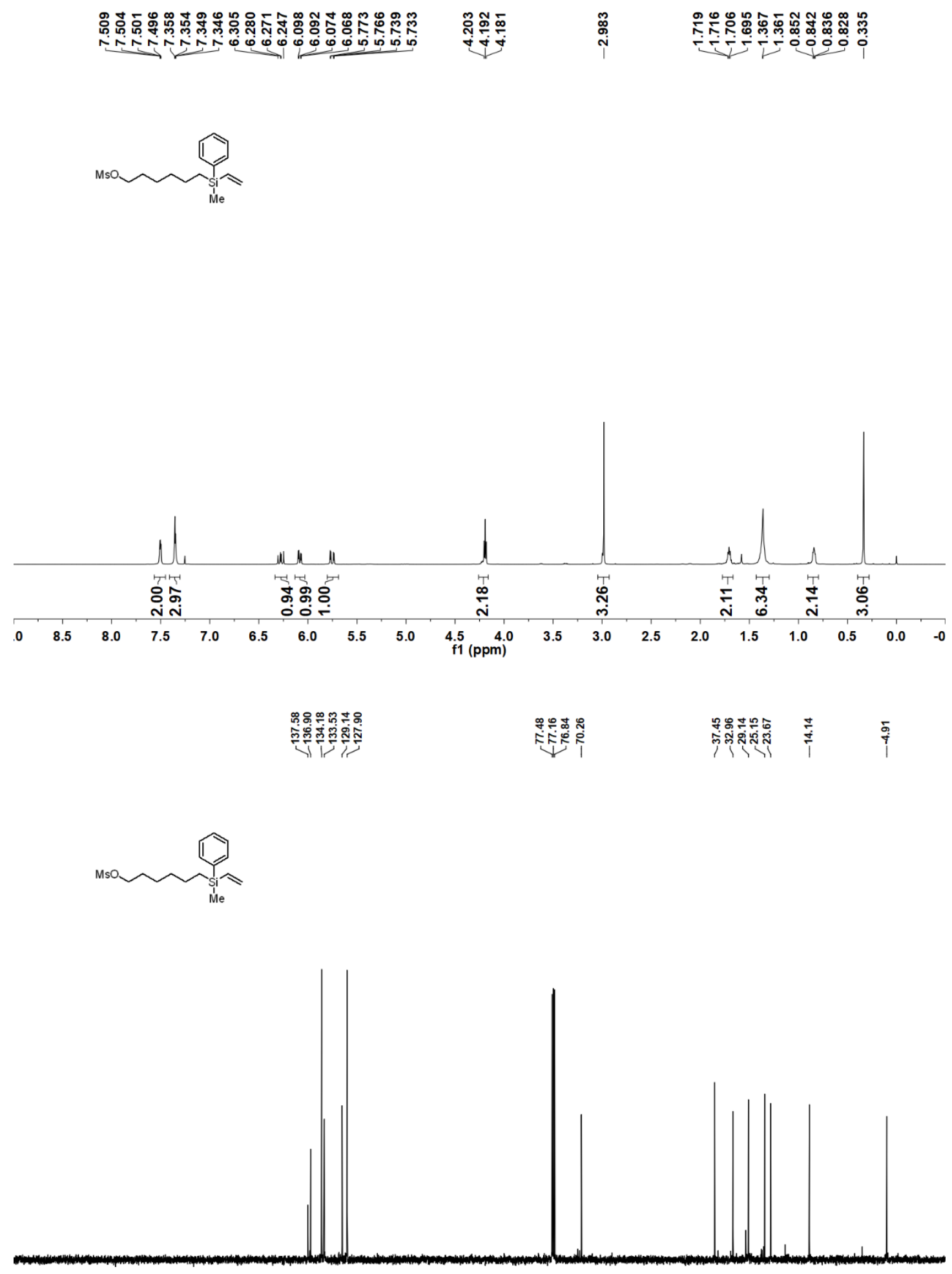

\begin{tabular}{|llllllllllllllllllllllll}
\hline 10 & 200 & 190 & 180 & 170 & 160 & 150 & 140 & 130 & 120 & 110 & $\underset{f 1}{100}(\mathrm{ppm})$ & 80 & 70 & 60 & 50 & 40 & 30 & 20 & 10 & 0 & -10
\end{tabular} 
3k; ${ }^{29} \mathrm{Si}$ NMR (79 MHz, CDCl3)

๑ே.
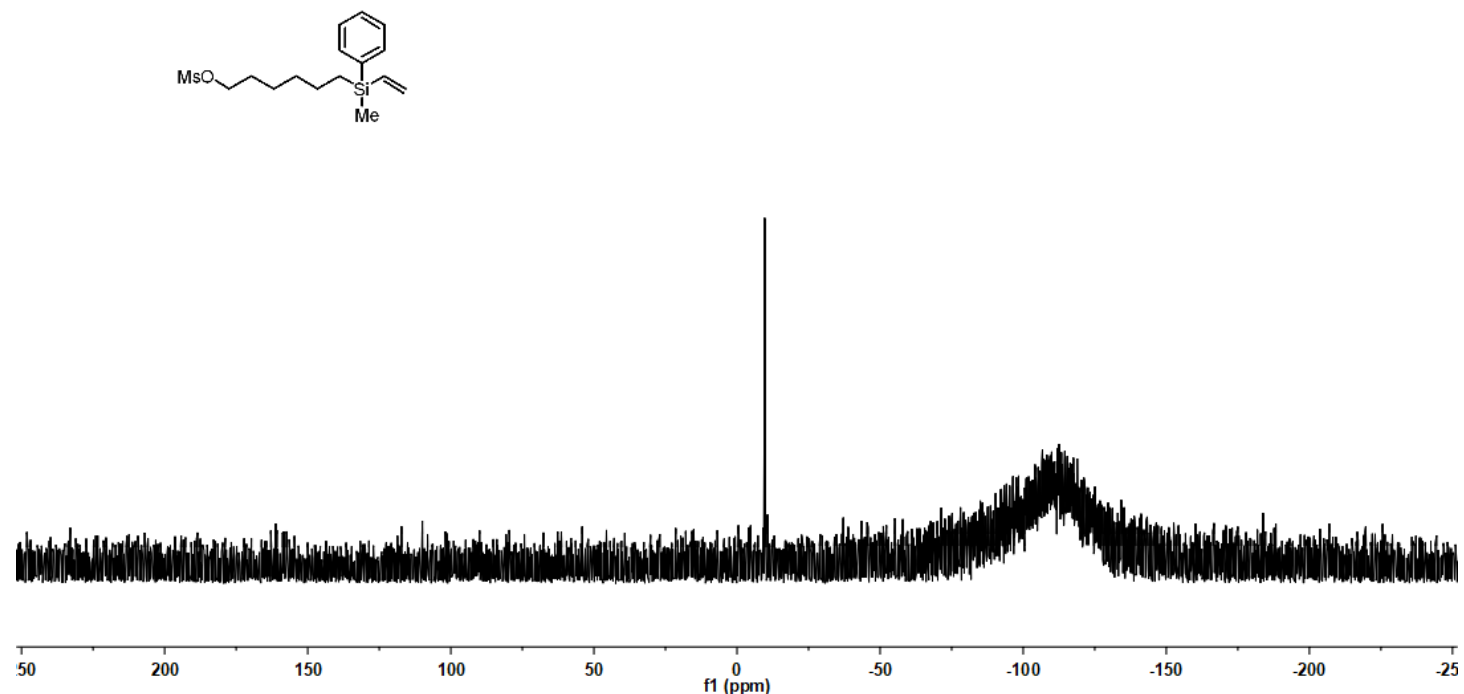
31; ${ }^{1} \mathrm{H}$ NMR (400 MHz, CDCl 3$) ;{ }^{13} \mathrm{C}$ NMR (100 MHz, CDCl $)$

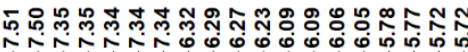

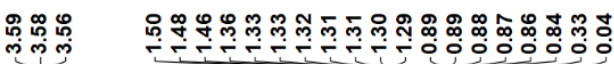
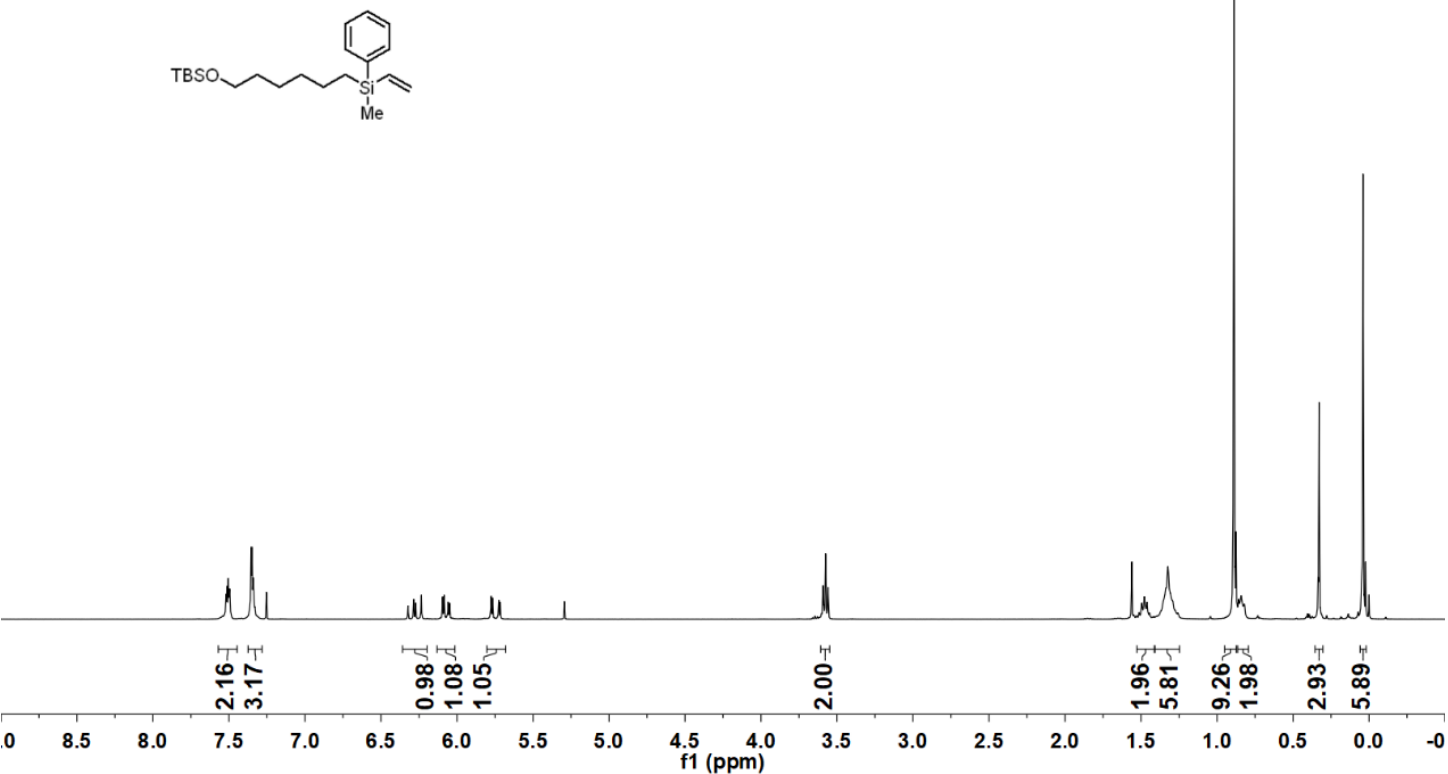

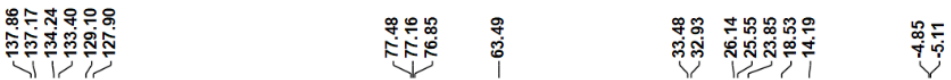

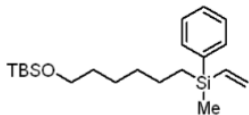

$\begin{array}{llllllllllll}10 & 200 & 190 & 180 & 170 & 160 & 150 & 140 & 130 & 120 & 110 & \begin{array}{c}100 \\ \mathrm{f} 1(\mathrm{ppm})\end{array}\end{array}$ 
31; ${ }^{29} \mathrm{Si}$ NMR (79 $\left.\mathrm{MHz}, \mathrm{CDCl}_{3}\right)$

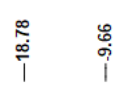
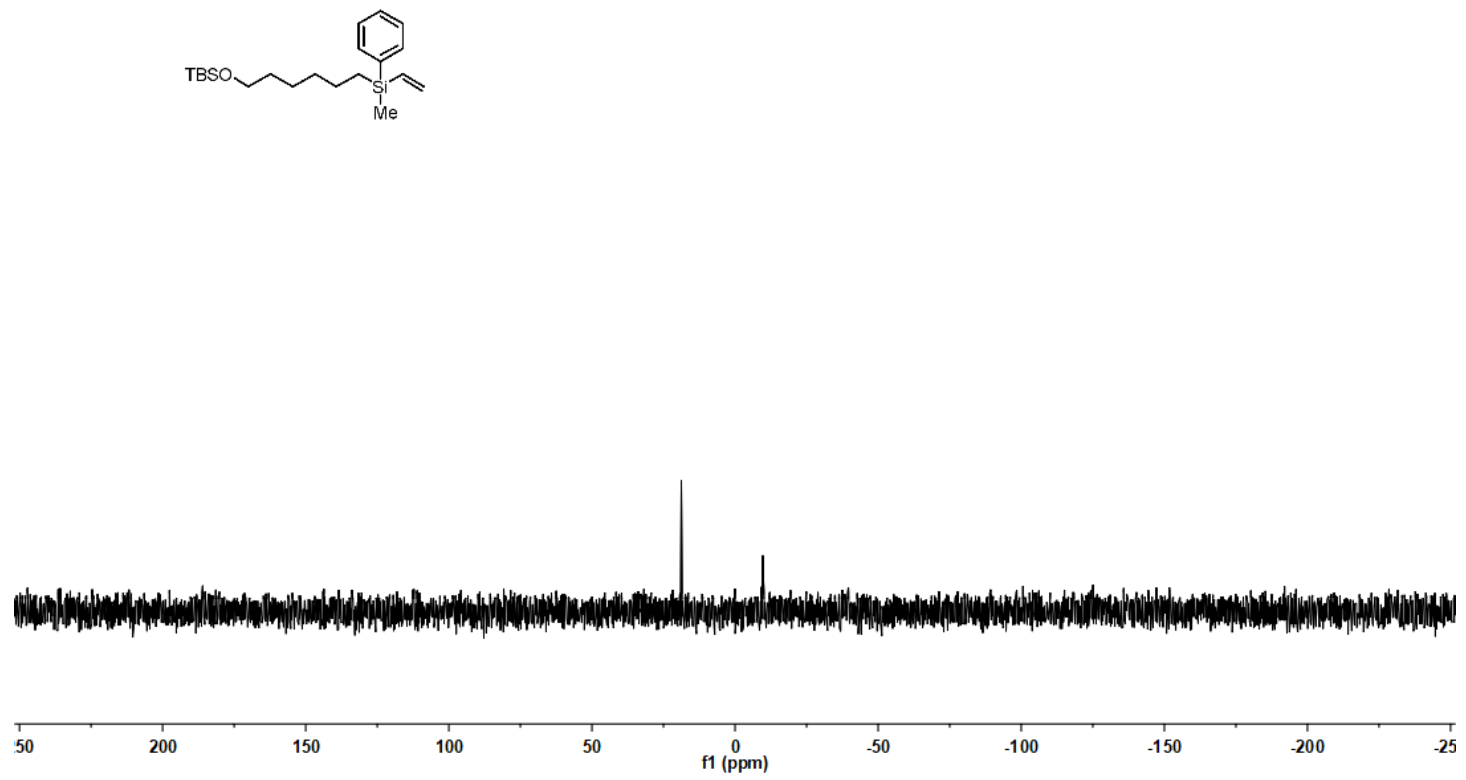
3m; ${ }^{1} \mathrm{H}$ NMR (400 MHz, $\left.\mathrm{CDCl}_{3}\right) ;{ }^{13} \mathrm{C} \mathrm{NMR}\left(100 \mathrm{MHz}, \mathrm{CDCl}_{3}\right)$

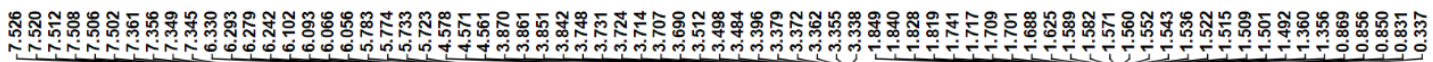
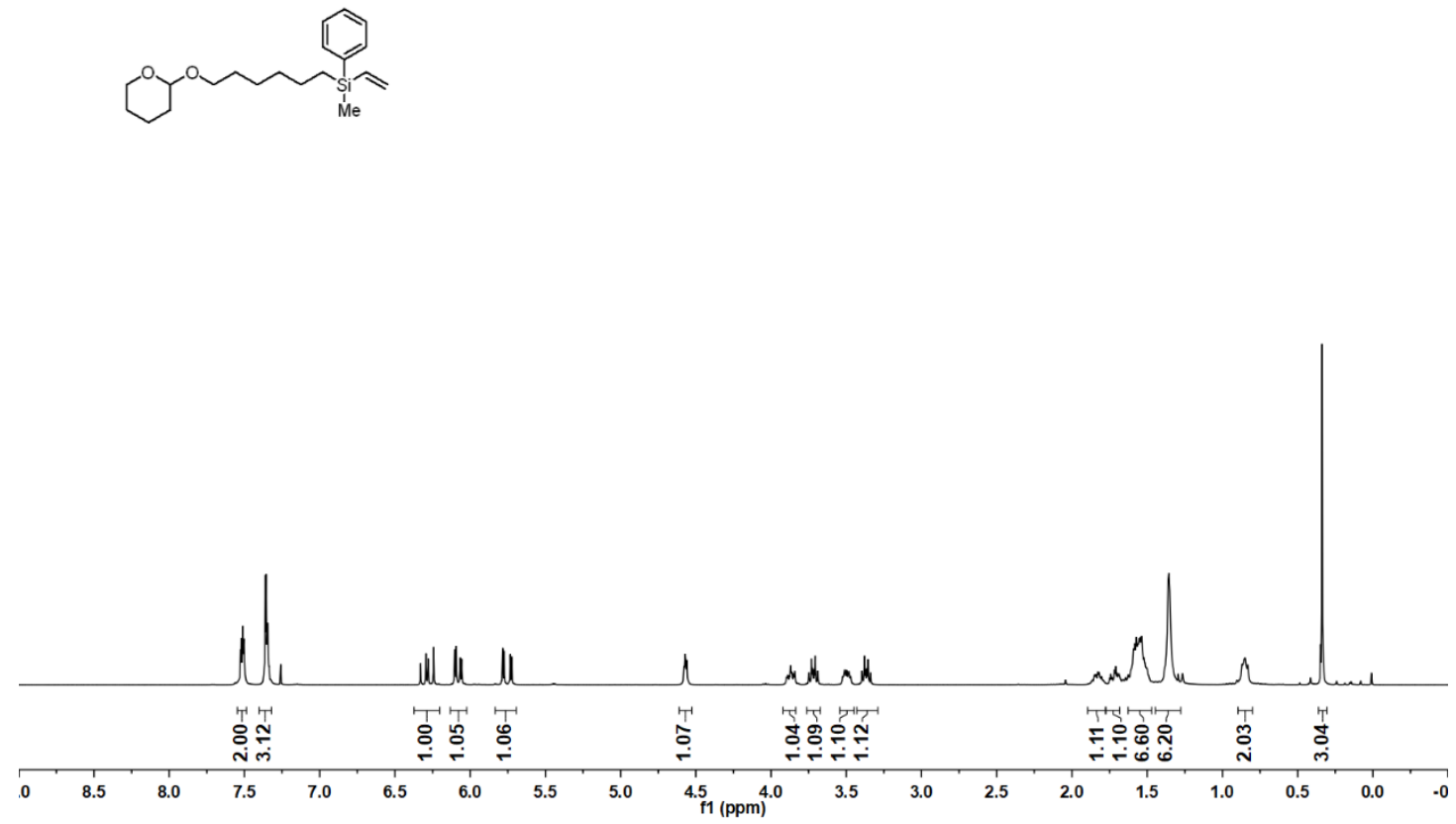

\begin{tabular}{|c|c|c|c|c|}
\hline 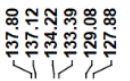 & $\begin{array}{l}\infty \\
\infty \\
\infty \\
\substack{j \\
j}\end{array}$ & 总鱼离 & 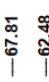 & 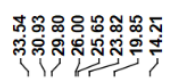 \\
\hline
\end{tabular}
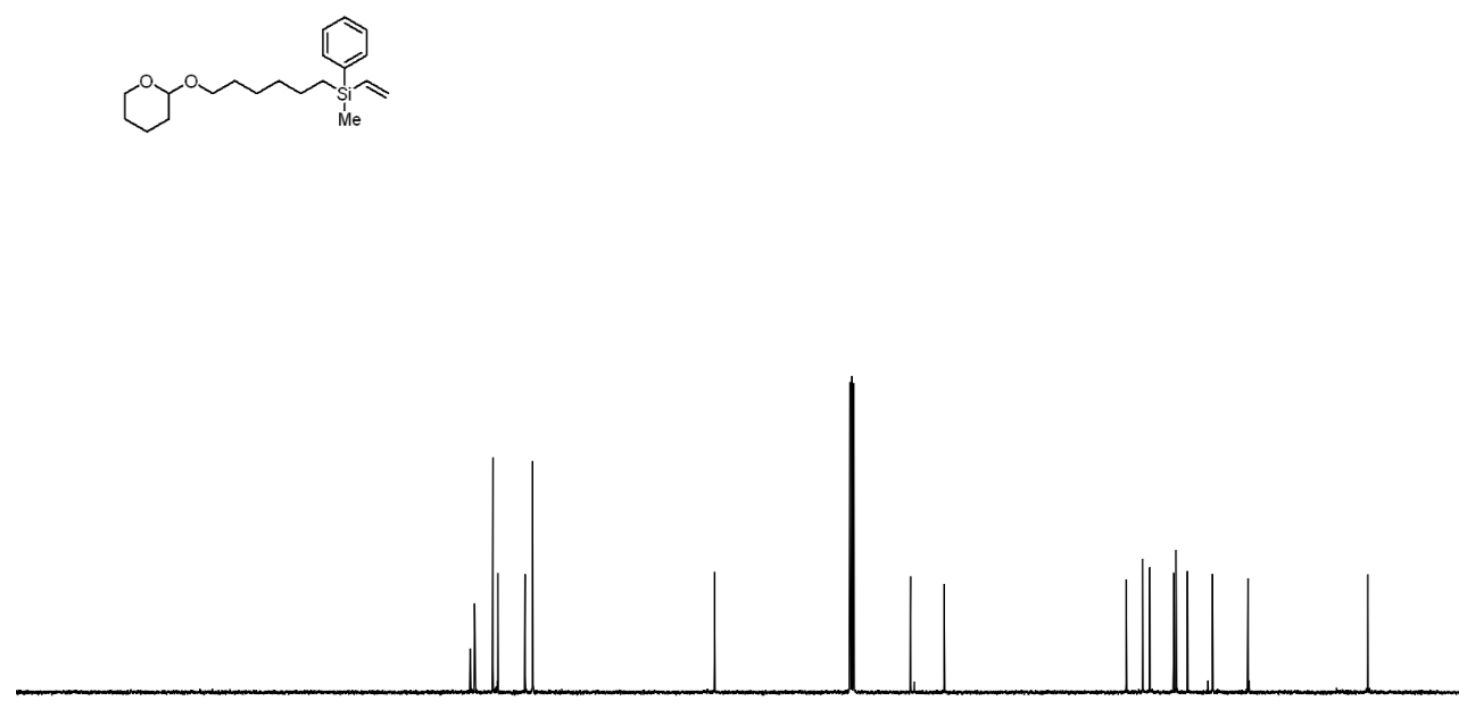

\begin{tabular}{lllllllllllll}
\hline 10 & 200 & 190 & 180 & 170 & 160 & 150 & 140 & 130 & 120 & 110 & $\underset{100}{10} 90$ \\
$\mathrm{f} 1(\mathrm{ppm})$
\end{tabular} 
3m; $\left.{ }^{29} \mathrm{Si} \mathrm{NMR} \mathrm{(79} \mathrm{MHz,} \mathrm{CDCl}_{3}\right)$

$\stackrel{\mathscr{Q}}{\mathrm{q}}$

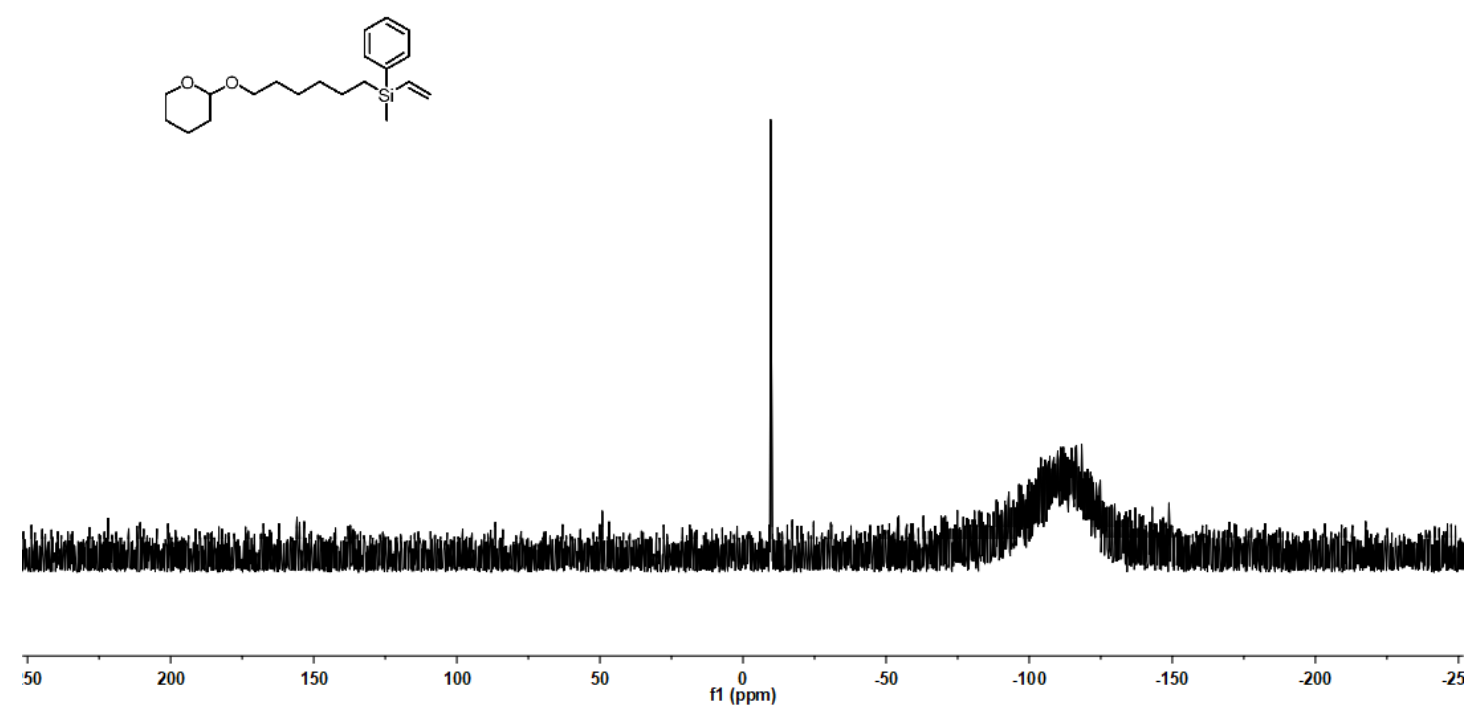


3n; ${ }^{1} \mathrm{H}$ NMR (400 MHz, CDCl 3$) ;{ }^{13} \mathrm{C}$ NMR (100 MHz, $\left.\mathrm{CDCl}_{3}\right)$

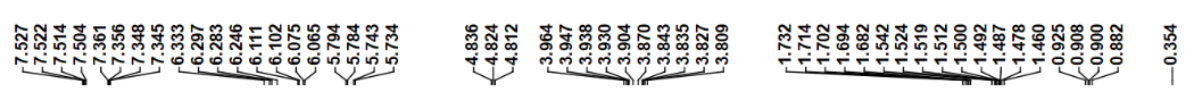
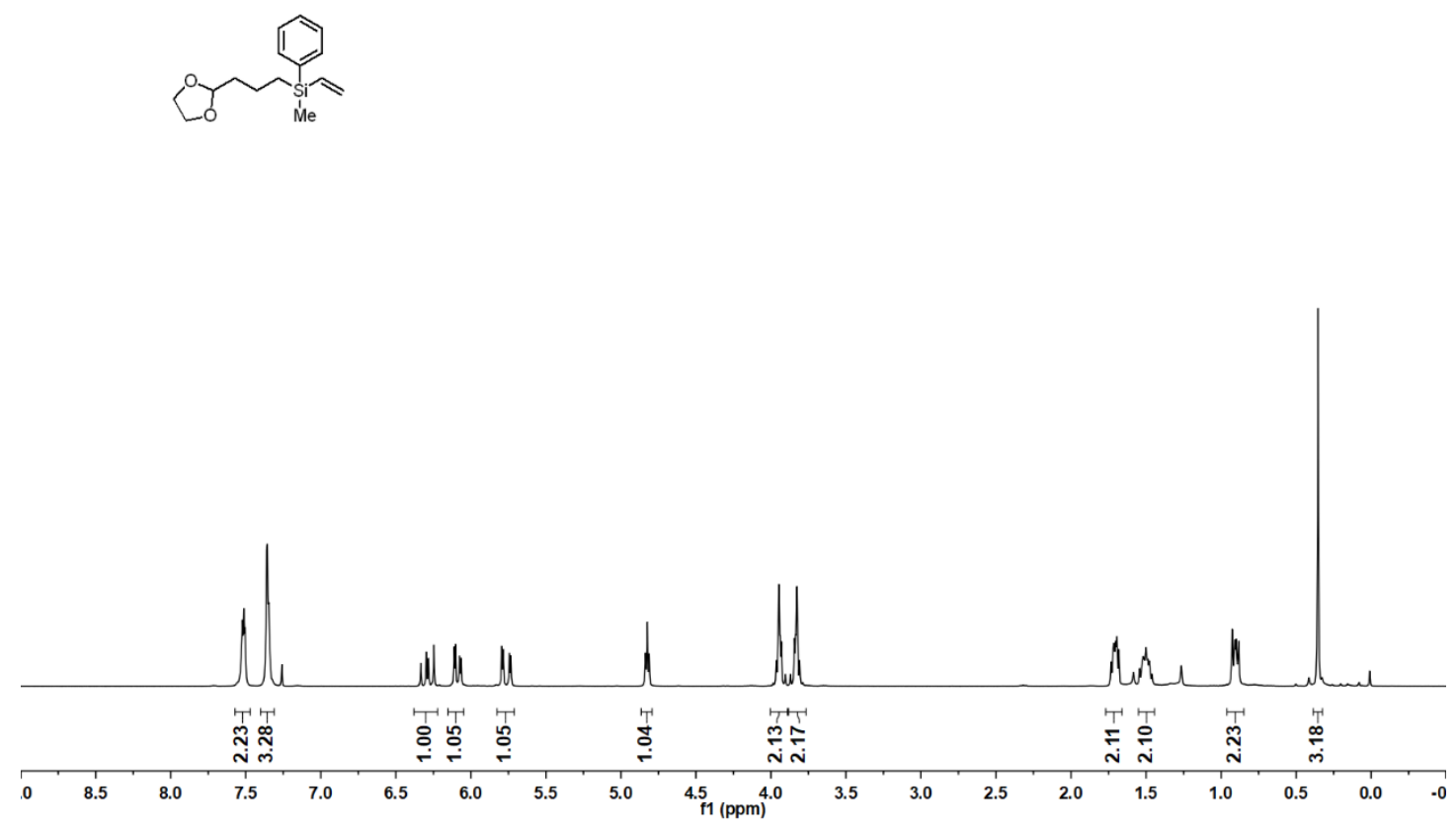

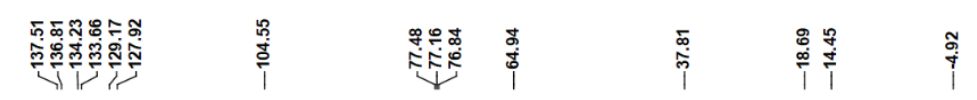
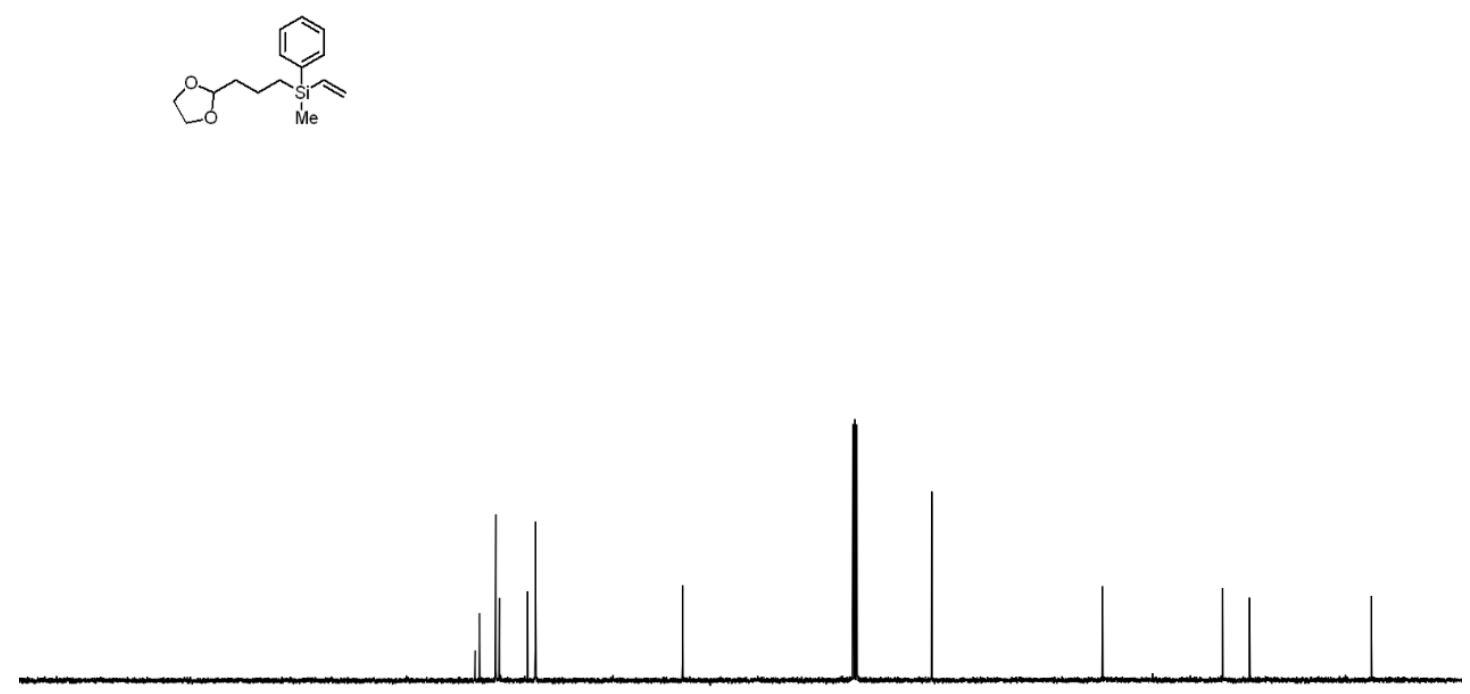

\begin{tabular}{|llllllllllllllllllllll}
10 & 200 & 190 & 180 & 170 & 160 & 150 & 140 & 130 & 120 & 110 & $\underset{f 1}{100}(\mathrm{ppm})$ & 80 & 70 & 60 & 50 & 40 & 30 & 20 & 10 & 0 & -10
\end{tabular} 
3n; ${ }^{29} \mathrm{Si}$ NMR (79 MHz, CDCl$)$

$\stackrel{2}{0}$

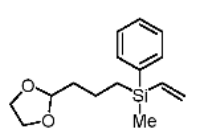

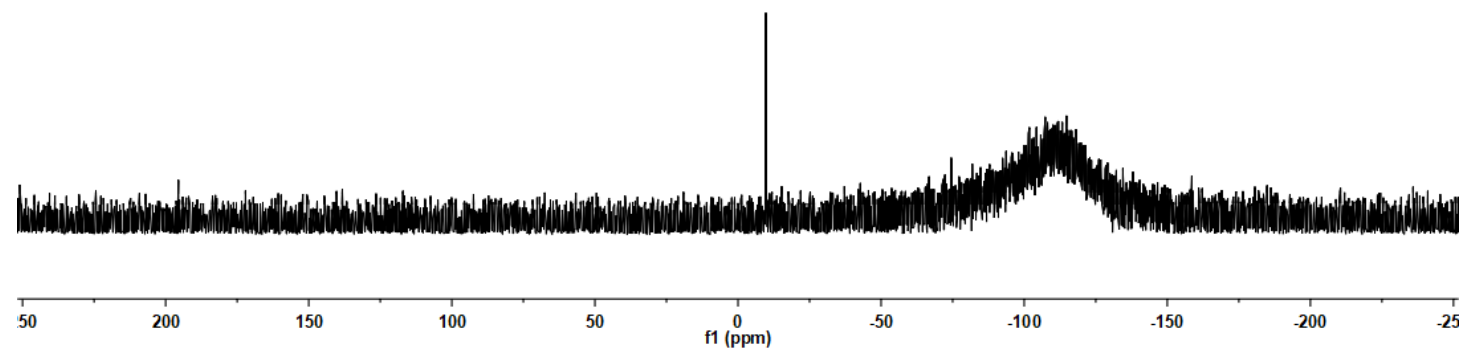


3o; ${ }^{1} \mathrm{H}$ NMR (400 MHz, $\left.\mathrm{CDCl}_{3}\right) ;{ }^{13} \mathrm{C}$ NMR (100 $\left.\mathrm{MHz}, \mathrm{CDCl}_{3}\right)$

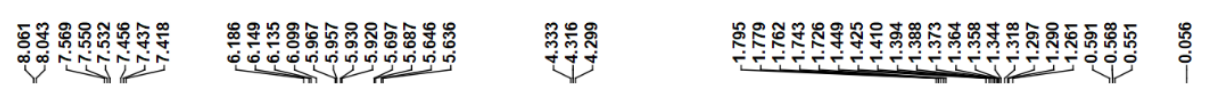

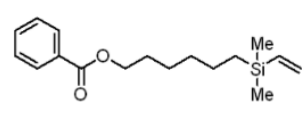

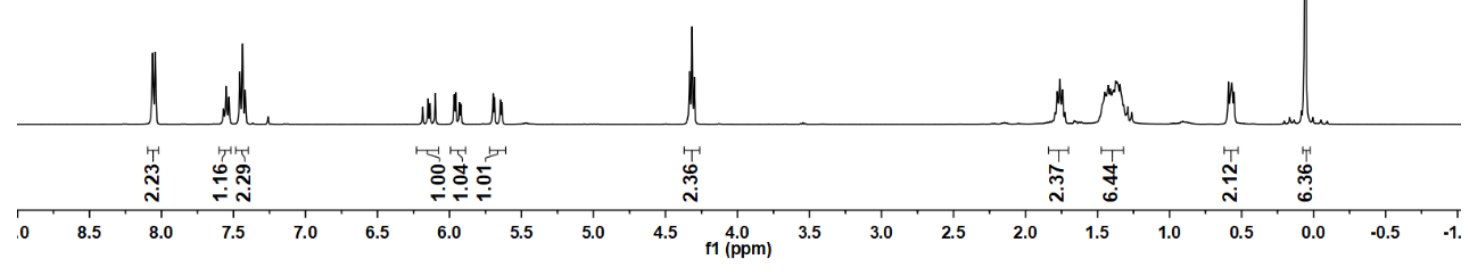

๕
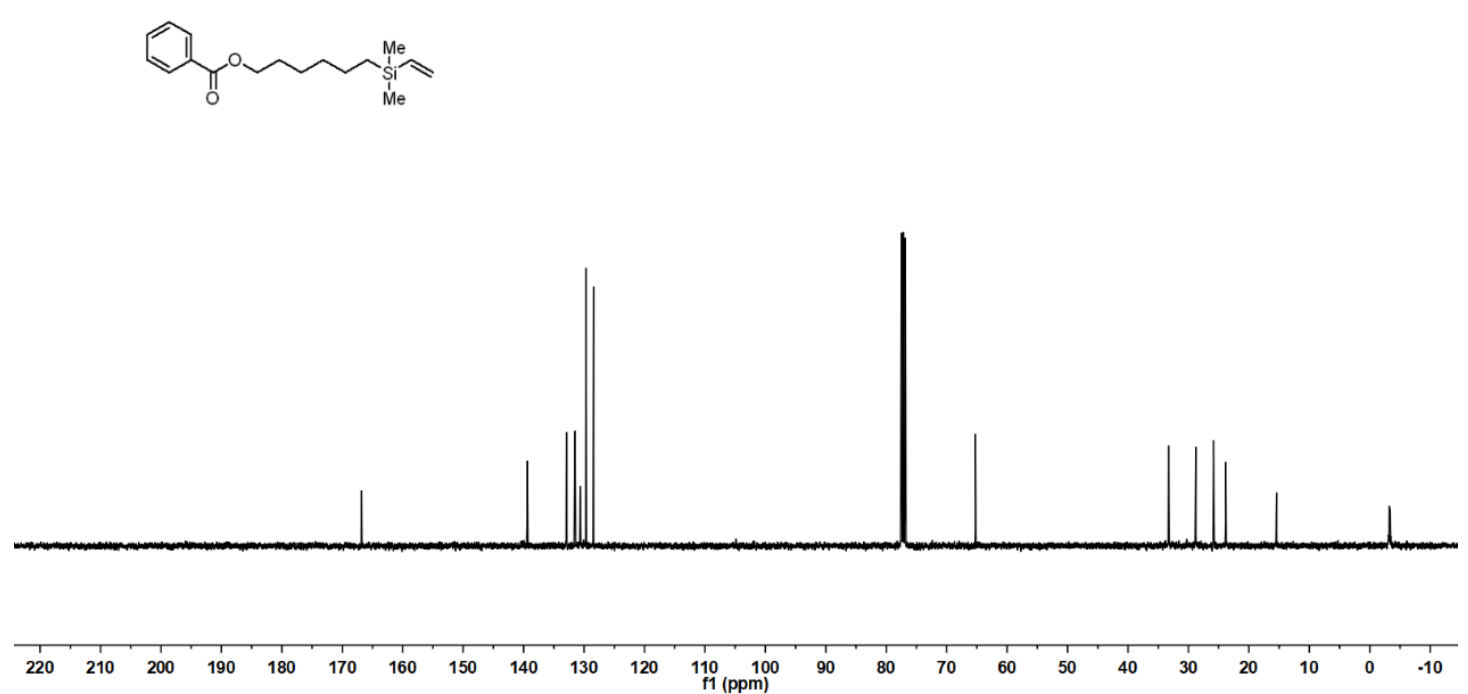
3o; $\left.{ }^{29} \mathrm{Si} \mathrm{NMR} \mathrm{(79} \mathrm{MHz,} \mathrm{CDCl} 3\right)$

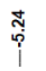

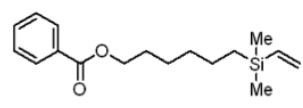

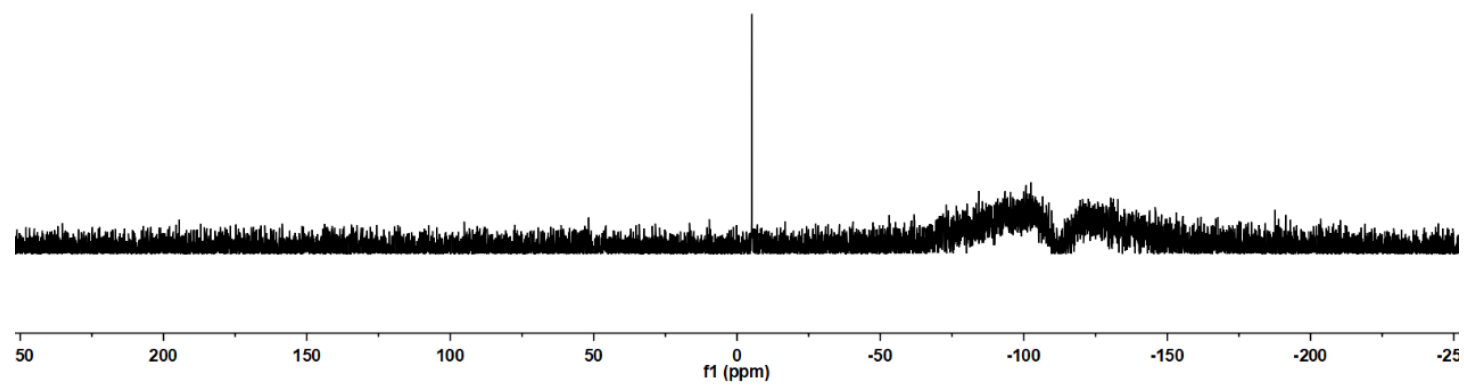


3p; ${ }^{1} \mathrm{H}$ NMR (400 MHz, CDCl $)$; ${ }^{13} \mathrm{C}$ NMR (100 $\left.\mathrm{MHz}^{\mathrm{CDCl}} 3\right)$

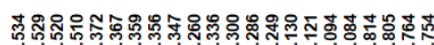

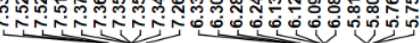

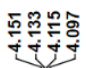

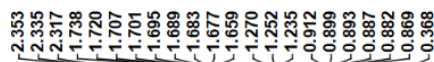

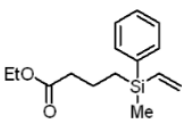

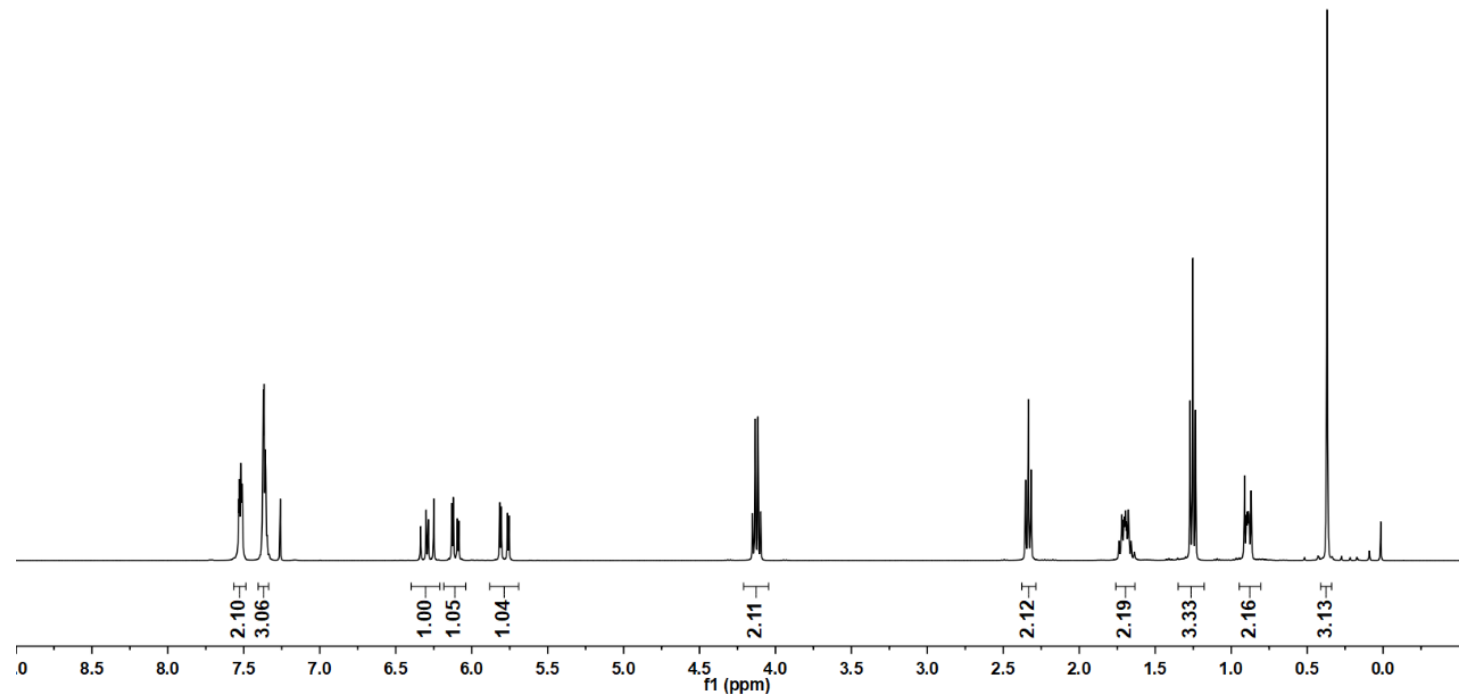

๕.

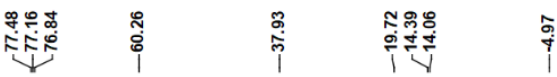
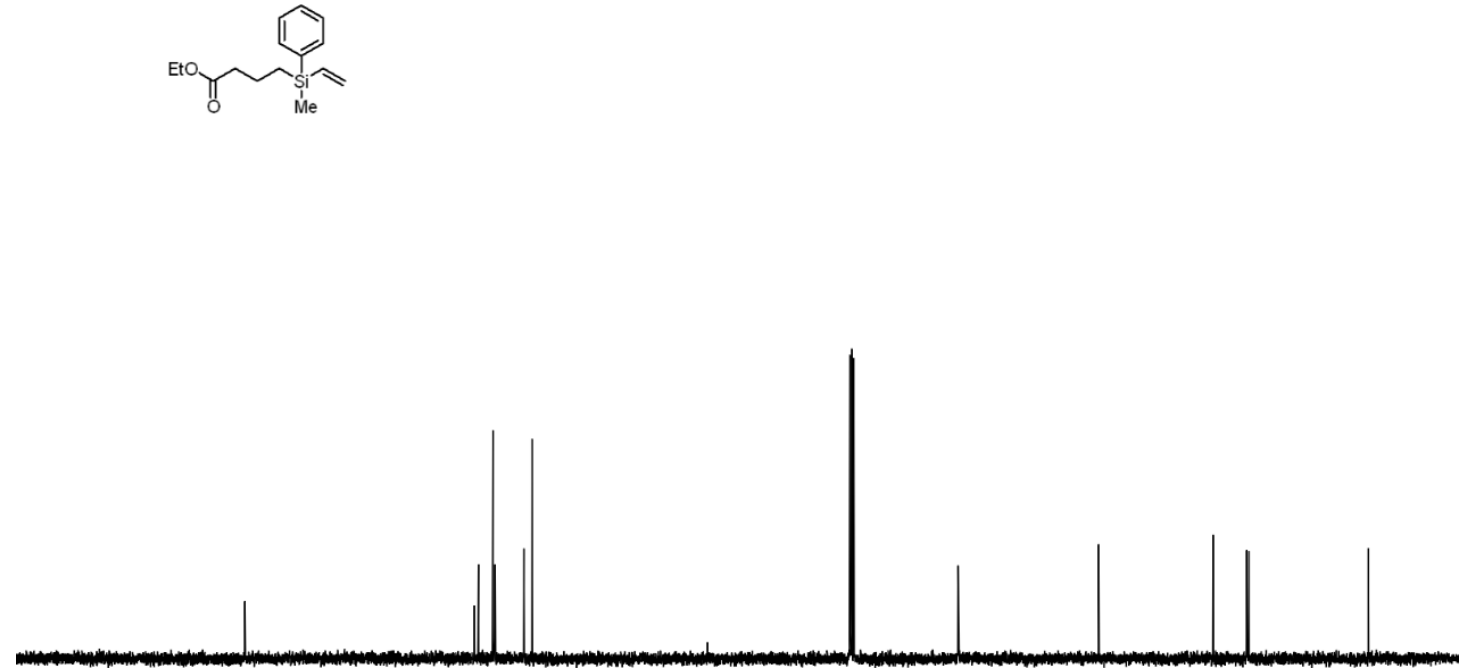

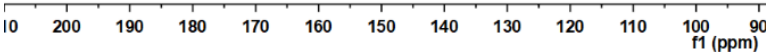


3p; ${ }^{29} \mathrm{Si}$ NMR (79 MHz, $\left.\mathrm{CDCl}_{3}\right)$

$\stackrel{2}{9}$
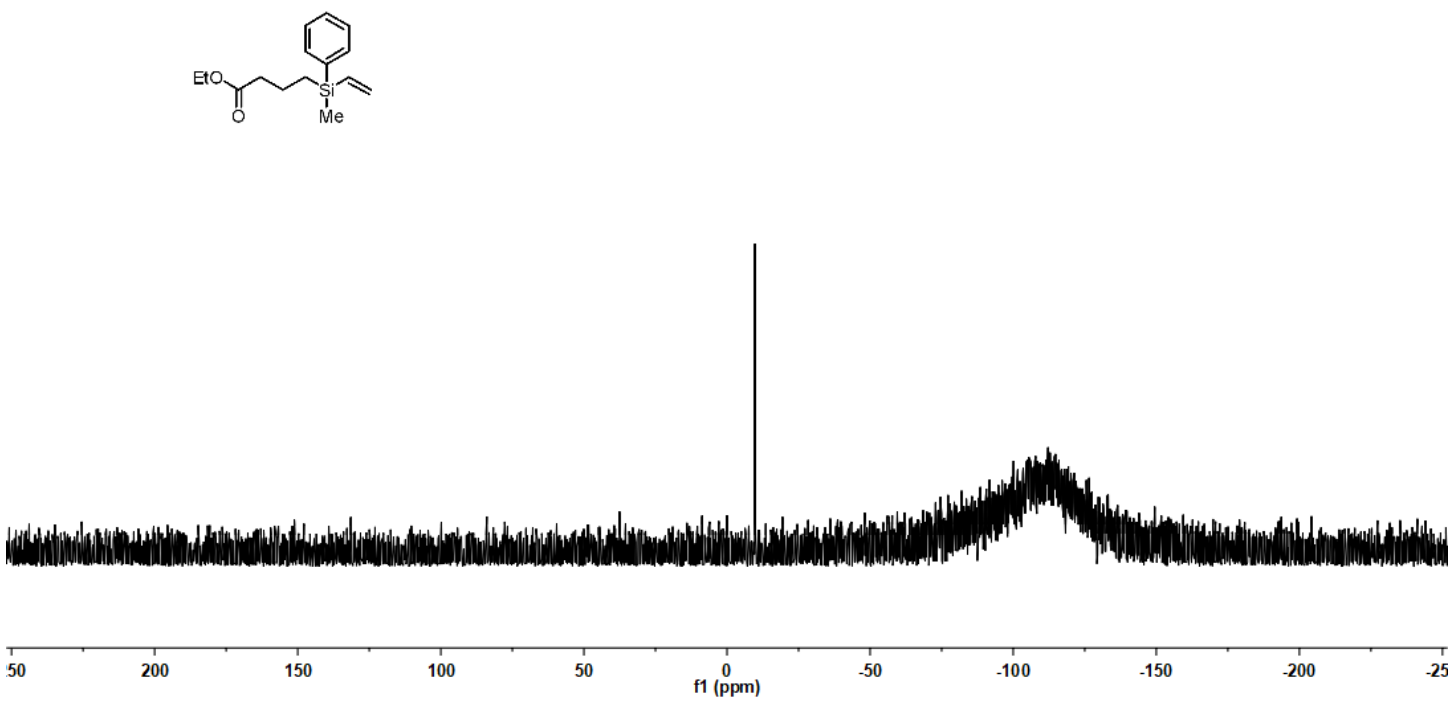

S64 
3q; ${ }^{1} \mathrm{H}$ NMR (400 MHz, $\left.\mathrm{CDCl}_{3}\right) ;{ }^{13} \mathrm{C} \mathrm{NMR}\left(100 \mathrm{MHz}, \mathrm{CDCl}_{3}\right)$

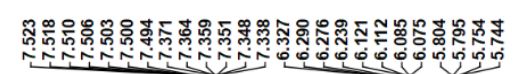

衡等
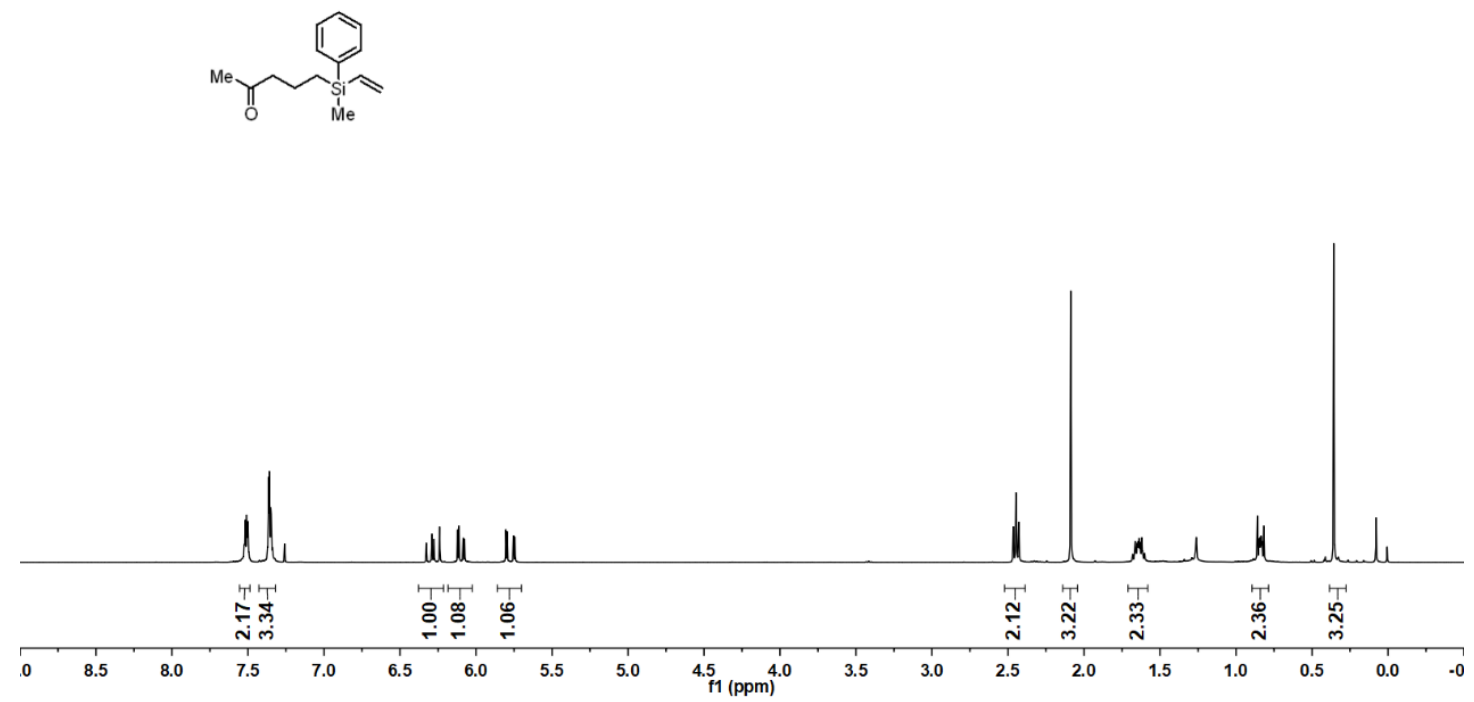

㶾<smiles>CC(C)CC[Si](C)(C)C</smiles>
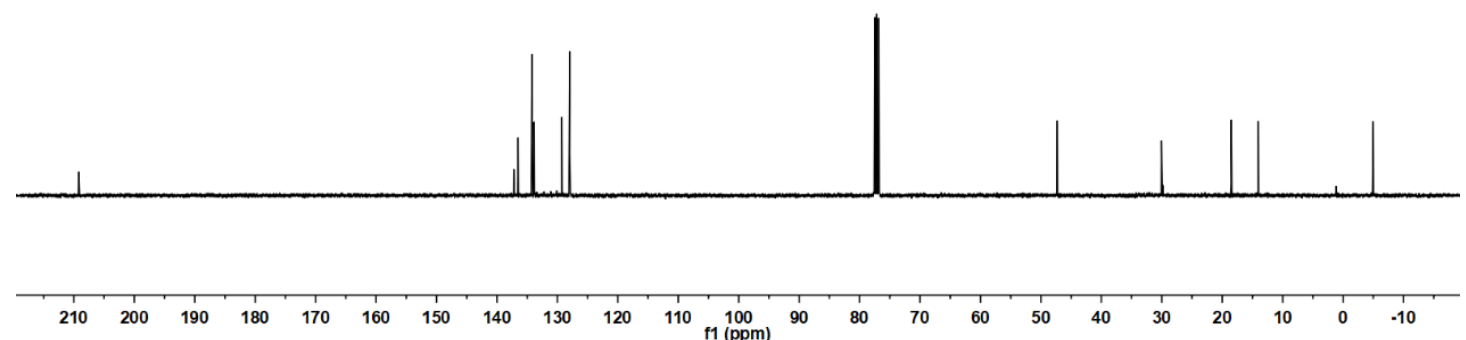
3q; $\left.{ }^{29} \mathrm{Si} \mathrm{NMR} \mathrm{(79} \mathrm{MHz,} \mathrm{CDCl}_{3}\right)$

$\stackrel{\infty}{0}$<smiles>CC(=O)CCC1(C)C=CC=C1</smiles>

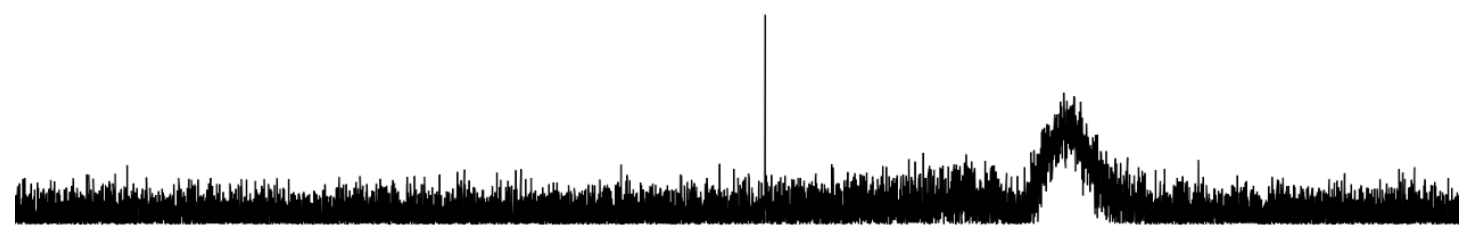

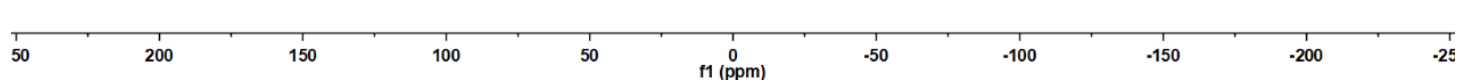


3r; ${ }^{1} \mathrm{H}$ NMR (400 MHz, CDCl 3$\left.) ;{ }^{13} \mathrm{C} \mathrm{NMR} \mathrm{(100} \mathrm{MHz,} \mathrm{CDCl} 3\right)$

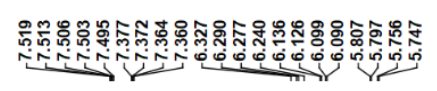

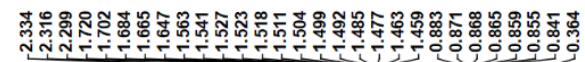
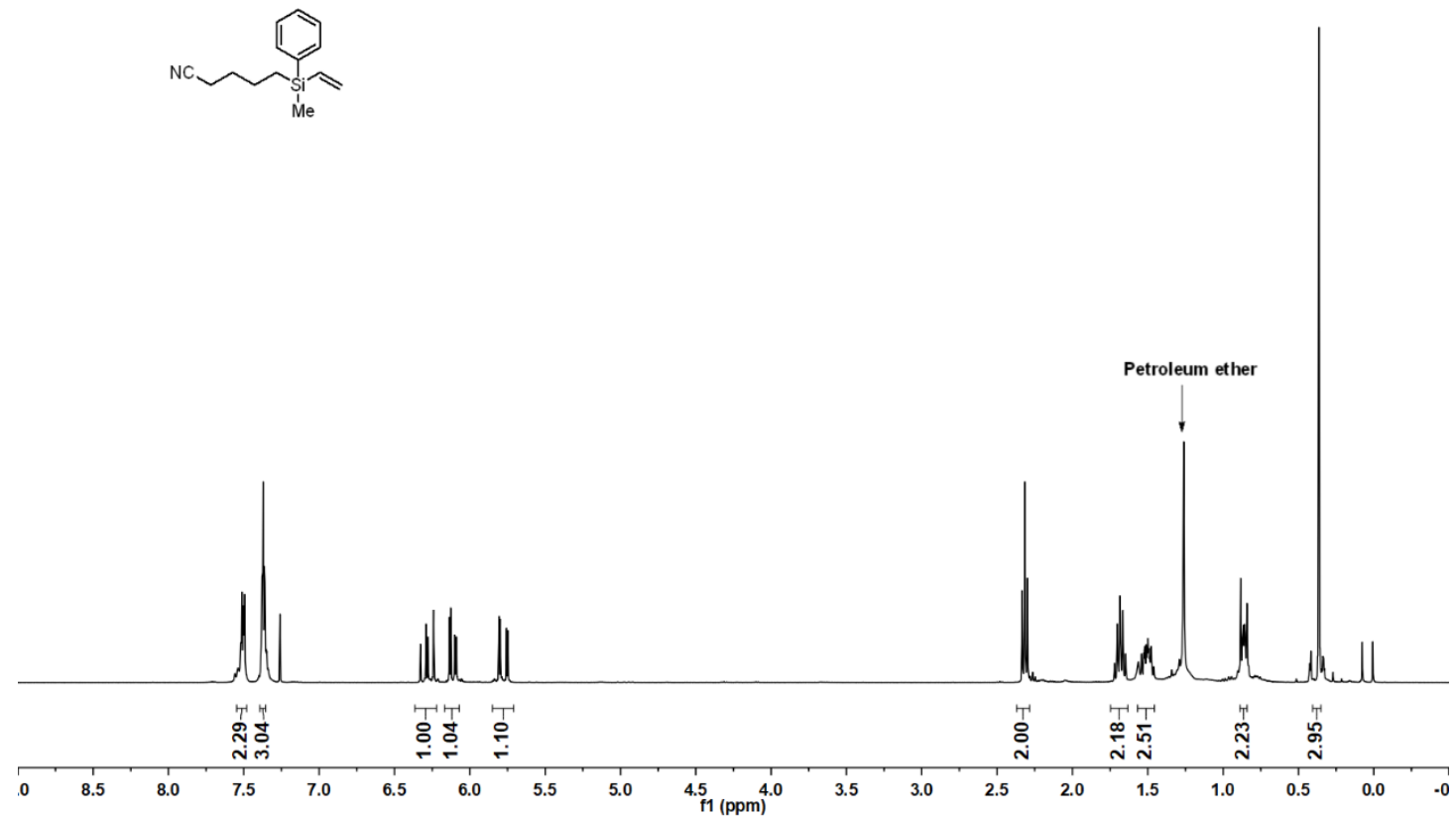

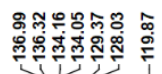

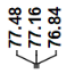

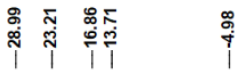
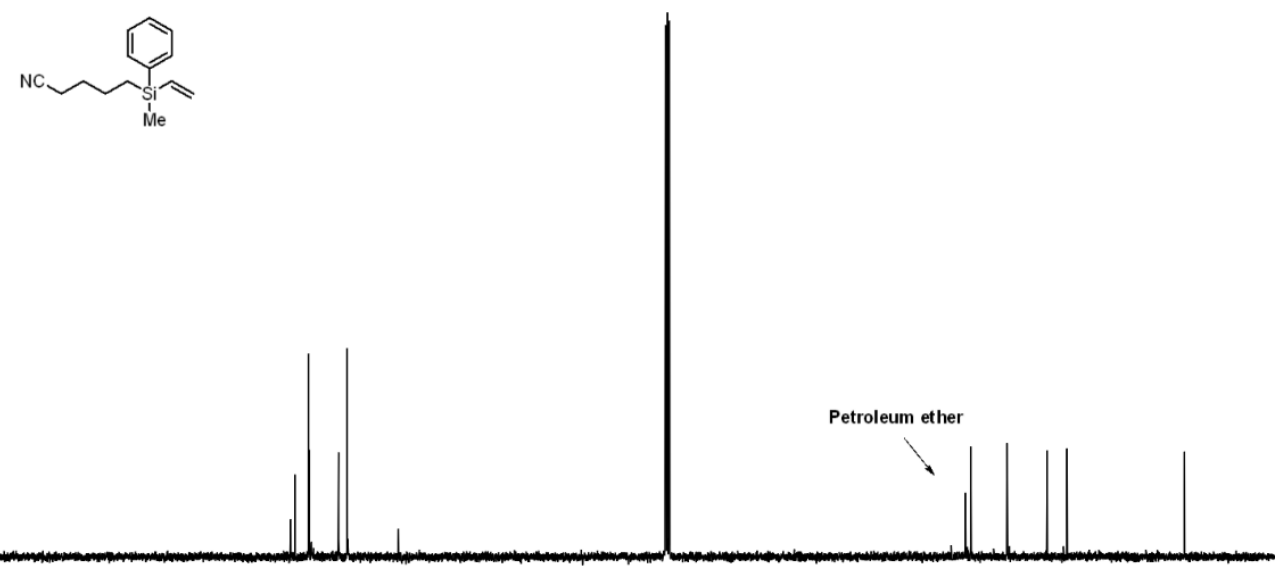

$\begin{array}{llllllllllll}10 & 200 & 190 & 180 & 170 & 160 & 150 & 140 & 130 & 120 & 110 & 100 \\ 11(\mathrm{ppm}) & 90\end{array}$ 8070

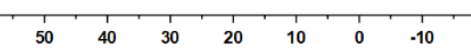


3r; ${ }^{29} \mathrm{Si}$ NMR (79 MHz, CDCl 3$)$

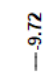<smiles>C=C[Si](C)(CCCCC#N)c1ccccc1</smiles>

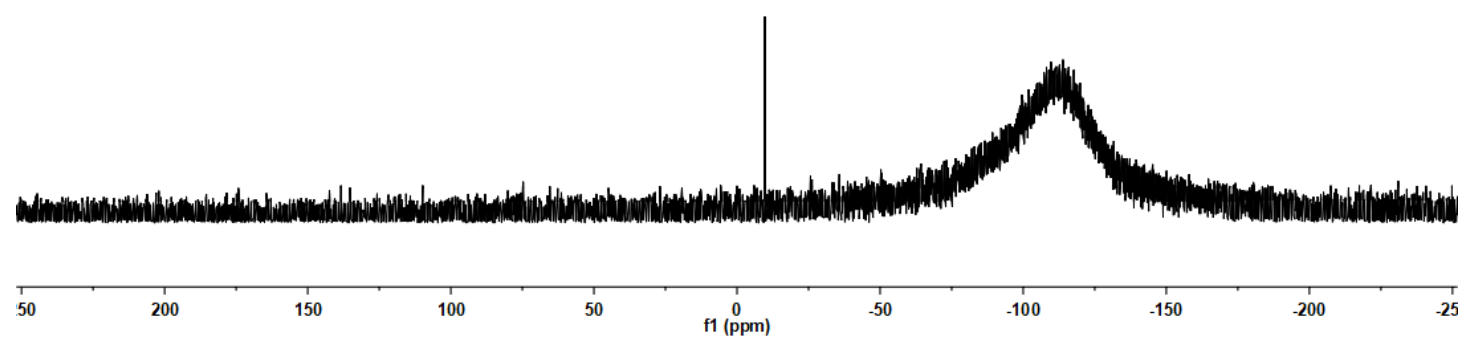


3s; ${ }^{1} \mathrm{H}$ NMR (400 MHz, CDCl 3$) ;{ }^{13} \mathrm{C}$ NMR (100 MHz, CDCl 3$)$

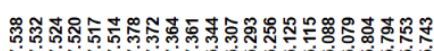

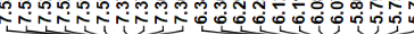

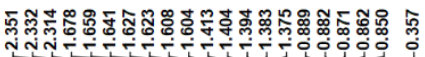

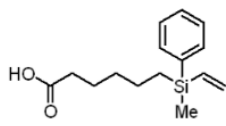

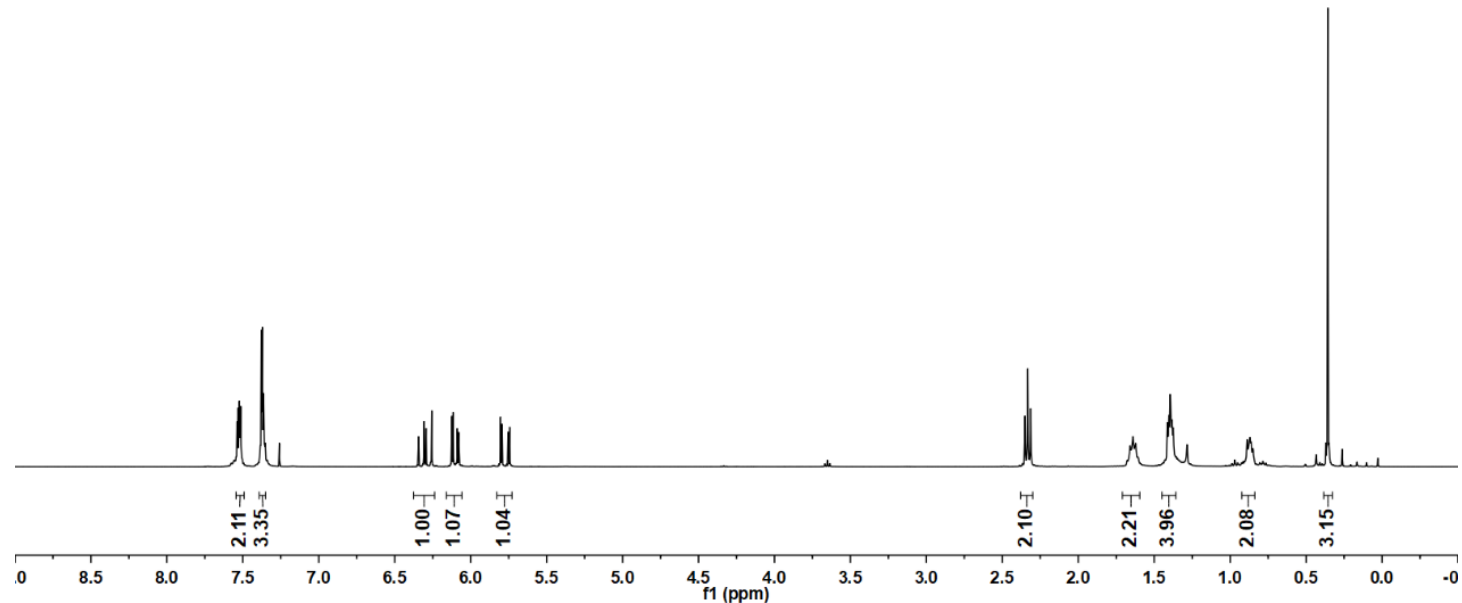

₹赵

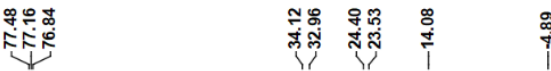
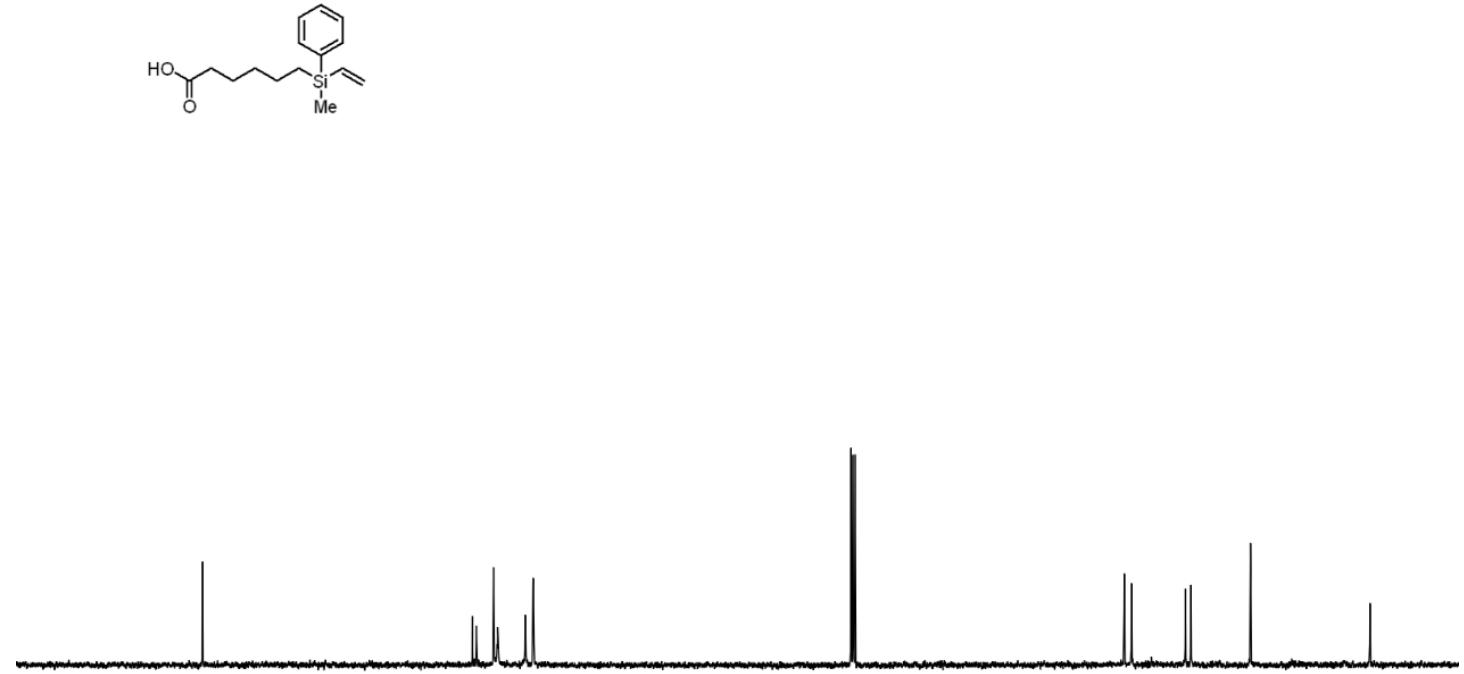

$\begin{array}{llllllllllll}10 & 200 & 190 & 180 & 170 & 160 & 150 & 140 & 130 & 120 & 110 & 100 \\ 11(\mathrm{ppm})\end{array}$ 
$3 s ;{ }^{29} \mathrm{Si}$ NMR (79 MHz, $\left.\mathrm{CDCl}_{3}\right)$

\section{$\stackrel{\square}{\circ}$}

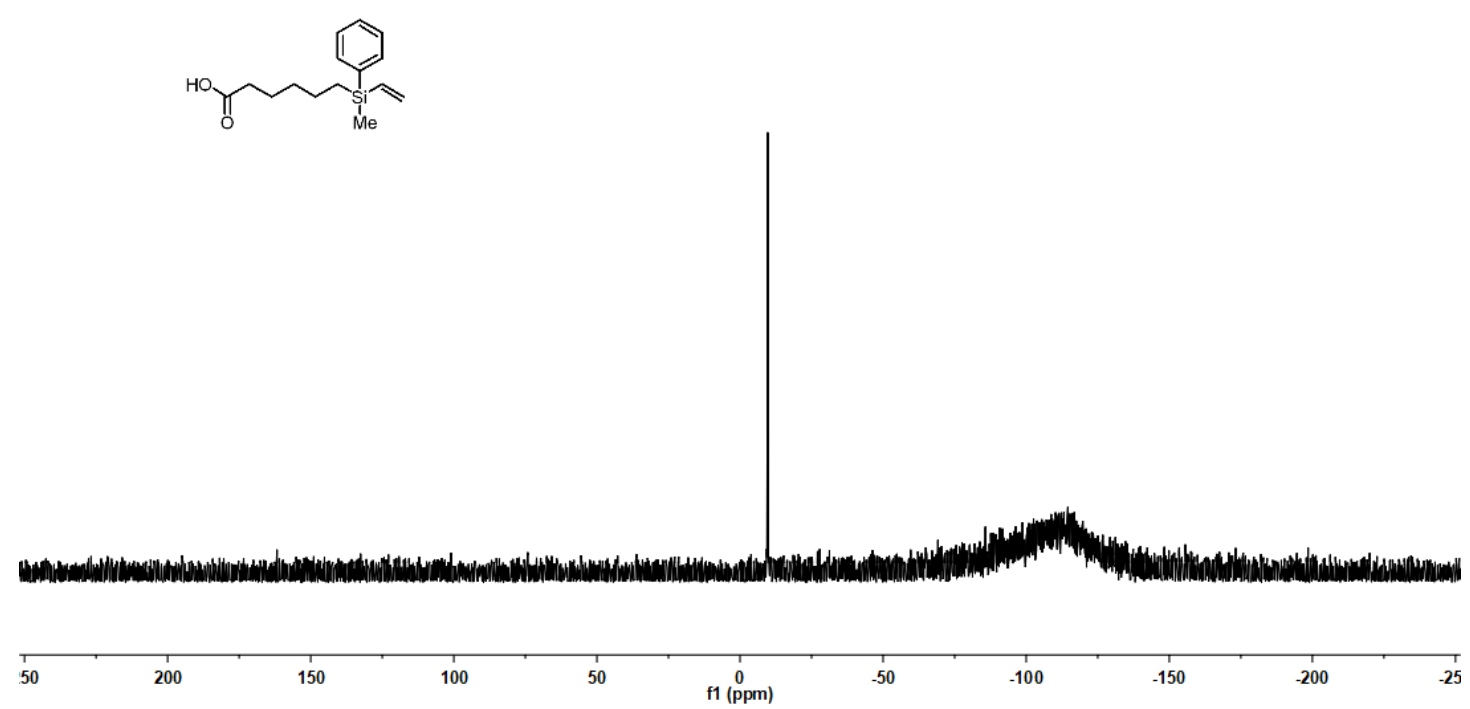


3t; ${ }^{1} \mathrm{H}$ NMR (600 MHz, CDCl $)$; ${ }^{13} \mathrm{C}$ NMR (150 MHz, CDCl $)$

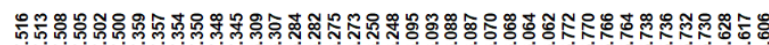

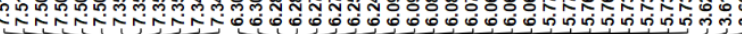

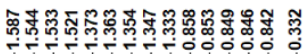
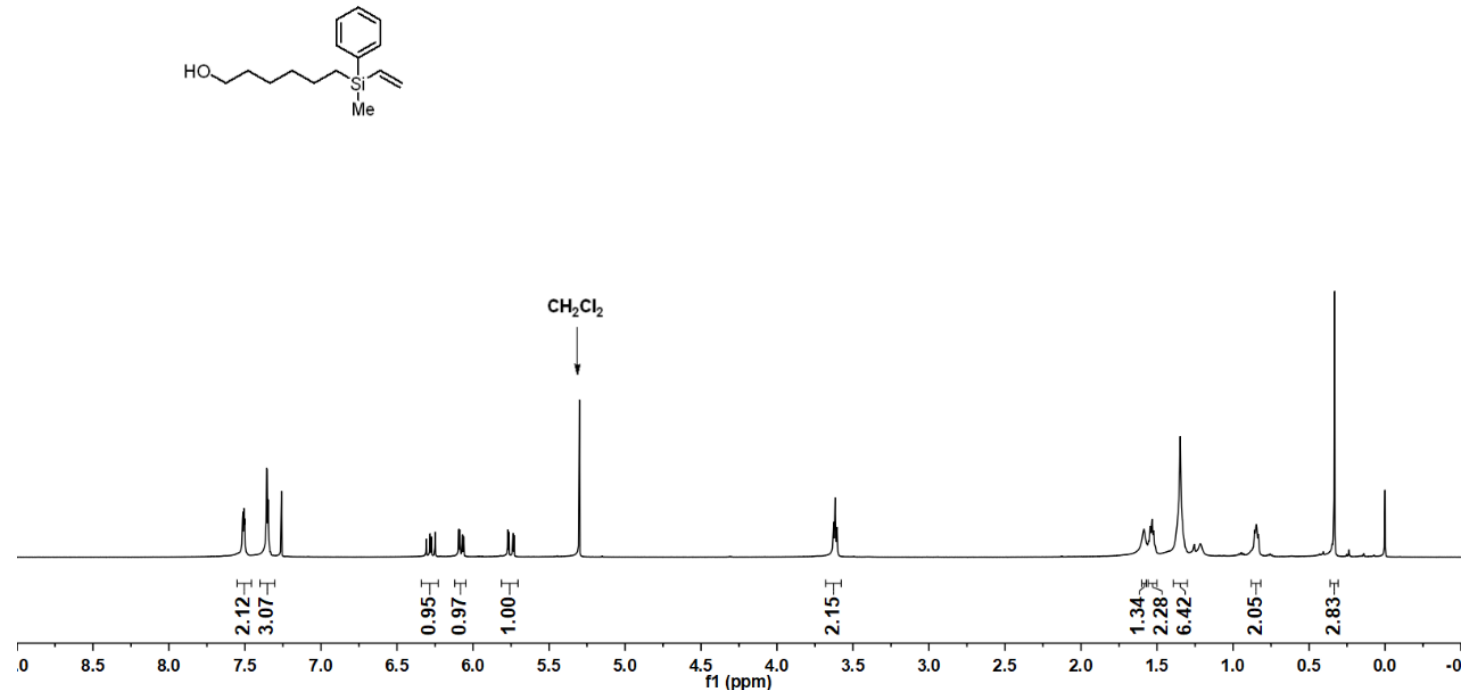

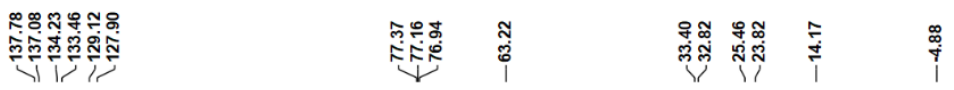
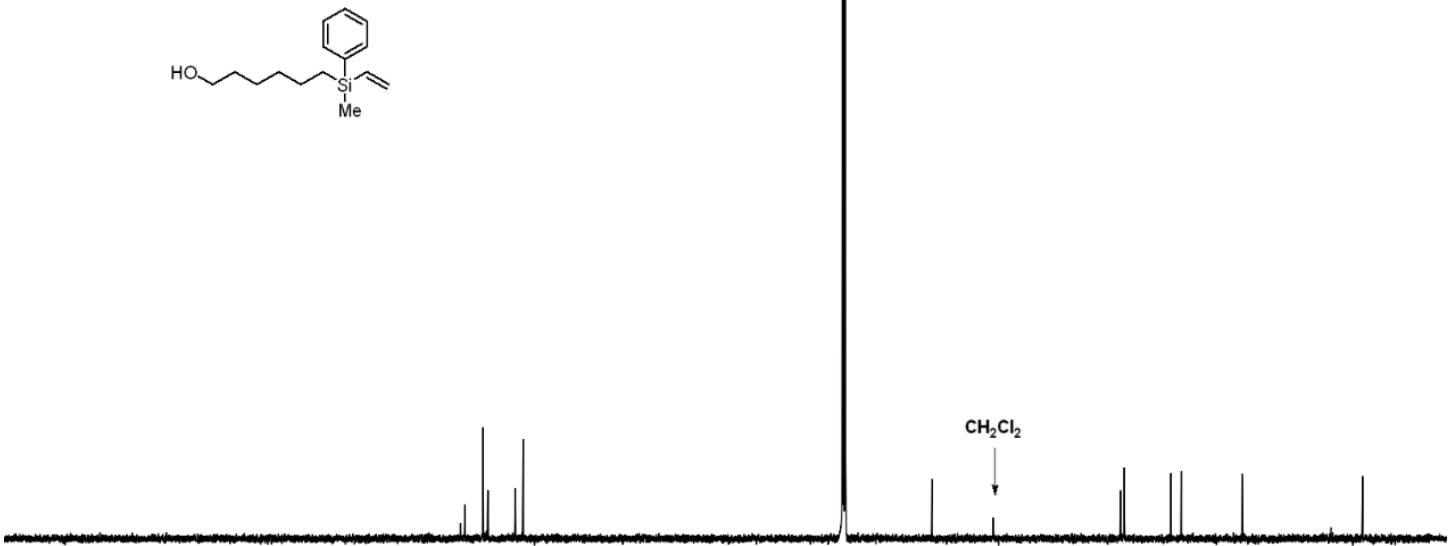

$\begin{array}{llllllllllll}10 & 200 & 190 & 180 & 170 & 160 & 150 & 140 & 130 & 120 & 110 & \begin{array}{l}100 \\ \mathrm{f} 1(\mathrm{ppm})\end{array}\end{array}$ 
3t; ${ }^{29} \mathrm{Si}$ NMR (79 MHz, $\mathrm{CDCl}_{3}$ )

$\stackrel{8}{i}$
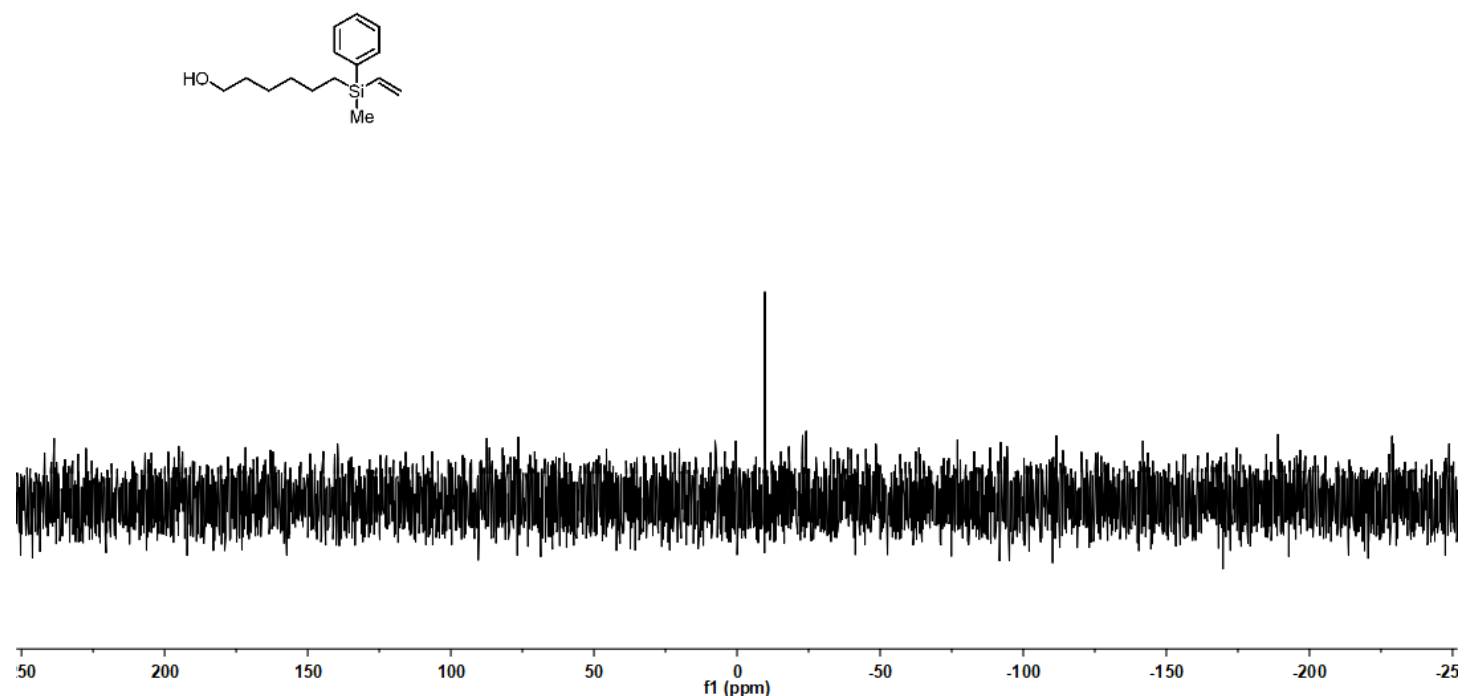
3u; ${ }^{1} \mathrm{H}$ NMR (400 MHz, $\left.\mathrm{CDCl}_{3}\right) ;{ }^{13} \mathrm{C} \mathrm{NMR}\left(100 \mathrm{MHz}, \mathrm{CDCl}_{3}\right)$

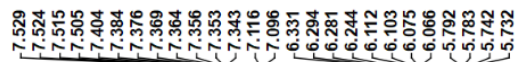

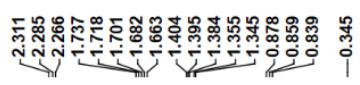
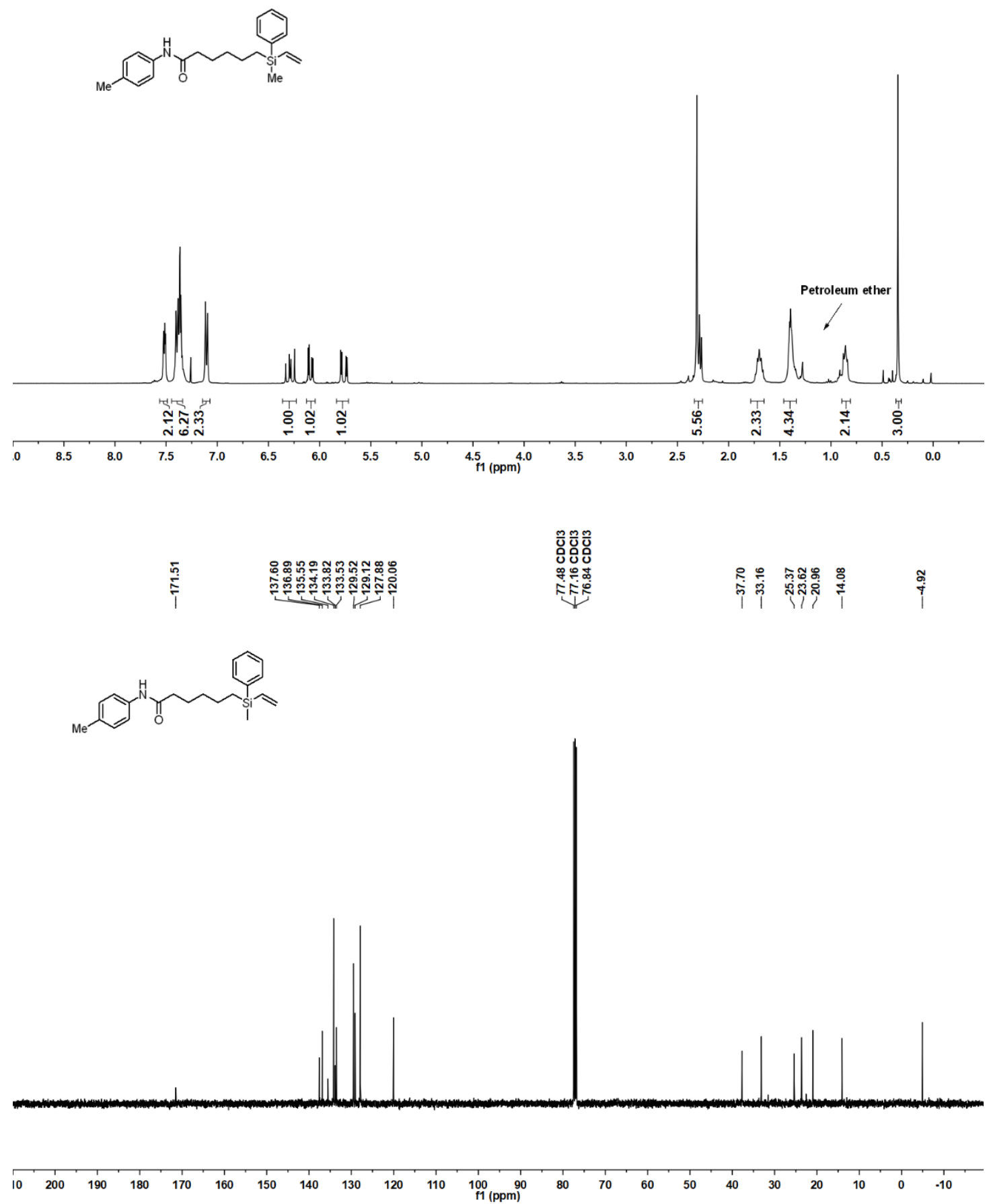
3u; ${ }^{29} \mathrm{Si} \mathrm{NMR} \mathrm{(79} \mathrm{MHz,} \mathrm{CDCl} 3$ )

苛

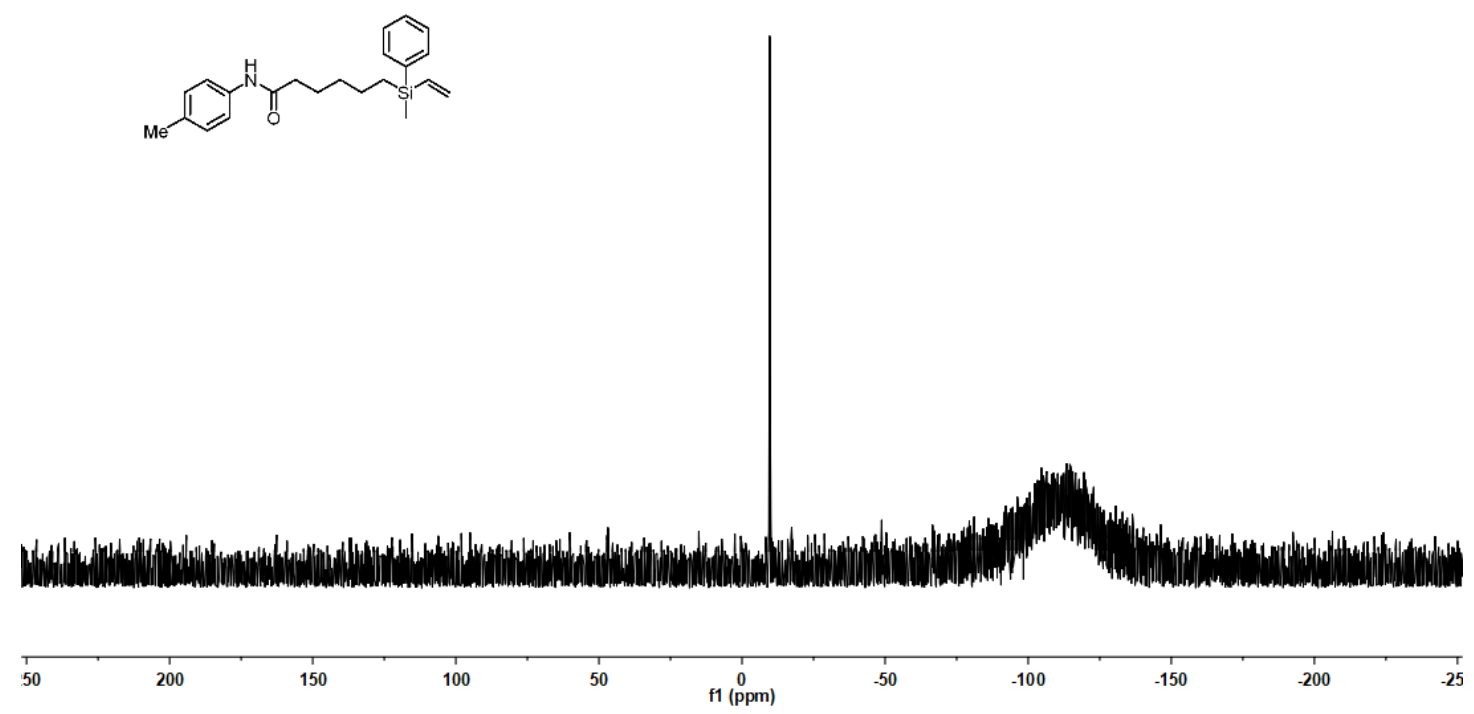


3v; ${ }^{1} \mathrm{H}$ NMR (400 MHz, $\left.\mathrm{CDCl}_{3}\right) ;{ }^{13} \mathrm{C} \mathrm{NMR}\left(100 \mathrm{MHz}, \mathrm{CDCl}_{3}\right)$

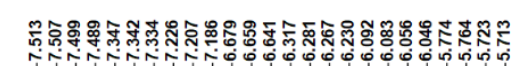

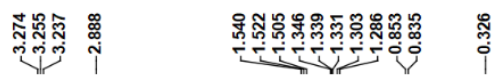

传

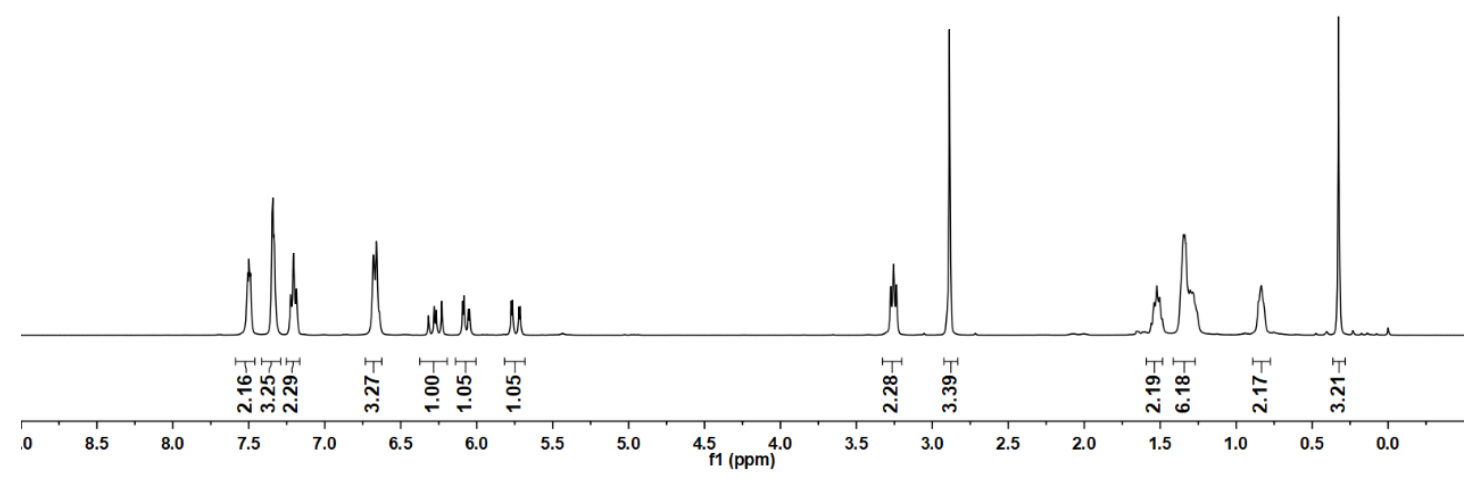

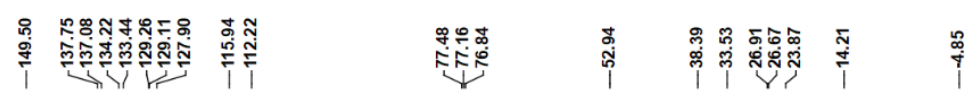
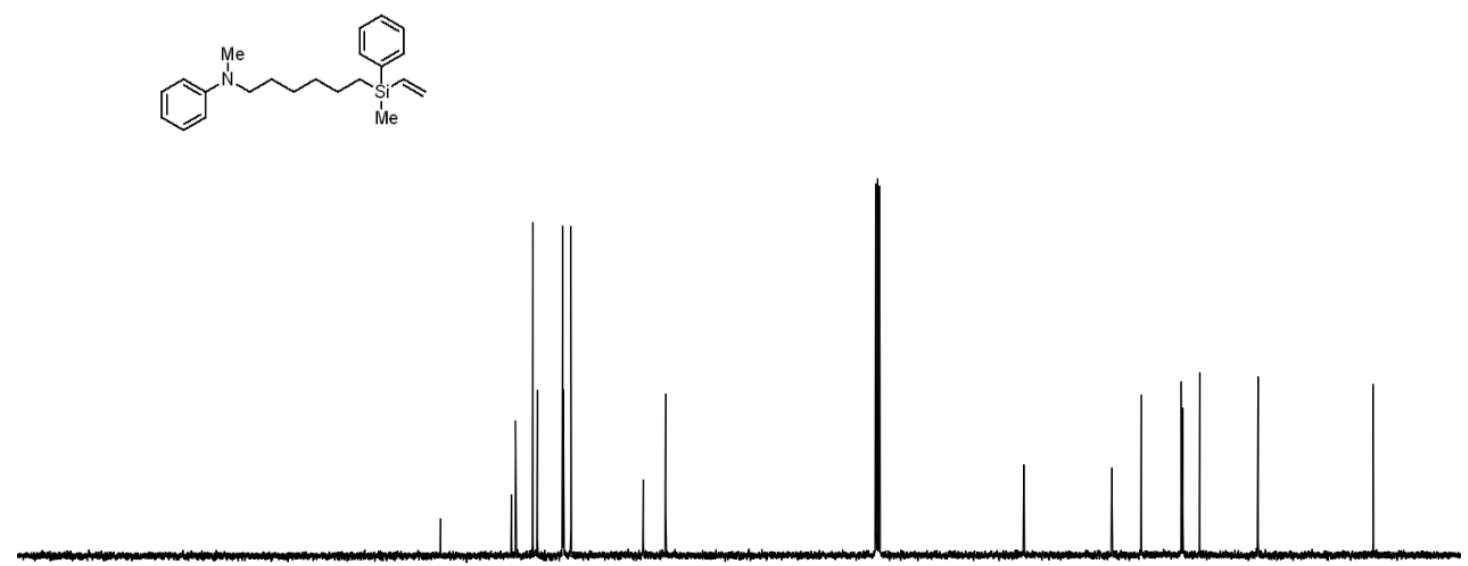

$\begin{array}{llllllllllllllllllllllllllllllll}210 & 200 & 190 & 180 & 170 & 160 & 150 & 140 & 130 & 120 & 110 & 100 & 90 & 80 & 70 & 60 & 50 & 40 & 30 & 20 & 10 & 0 & -10 & \end{array}$ 
3v; ${ }^{29} \mathrm{Si}$ NMR (79 MHz, CDCl 3$)$

๑ே

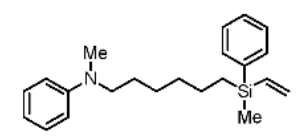

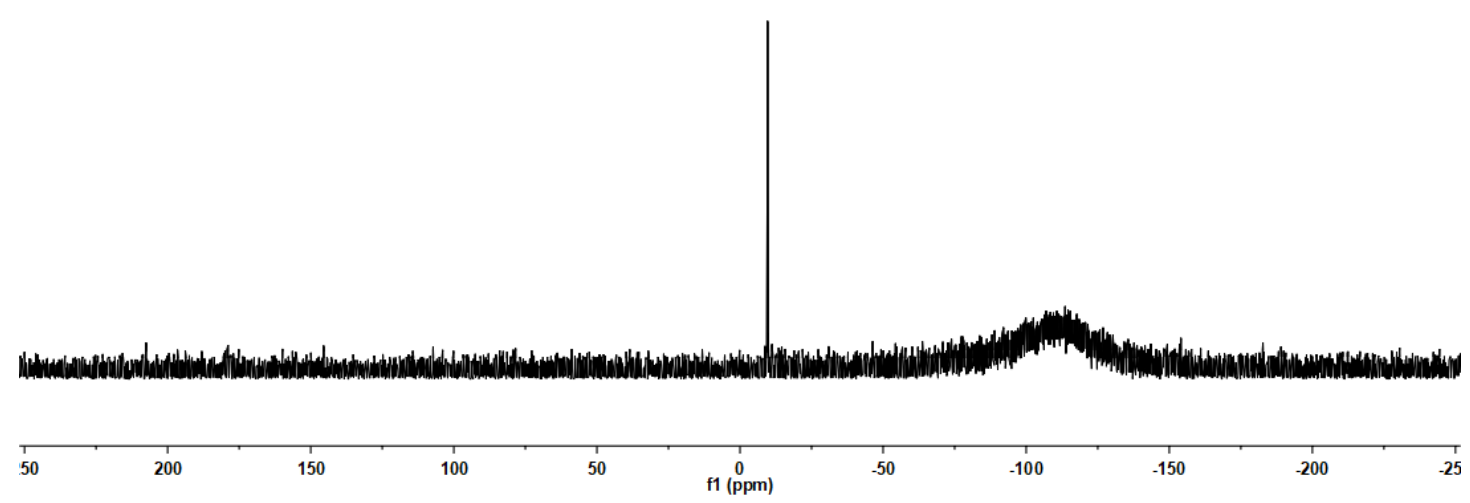


3w; ${ }^{1} \mathrm{H}$ NMR (400 MHz, CDCl 3$) ;{ }^{13} \mathrm{C}$ NMR (100 MHz, $\left.\mathrm{CDCl}_{3}\right)$

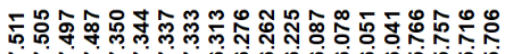

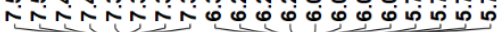

ำ

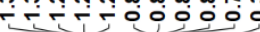

(1)

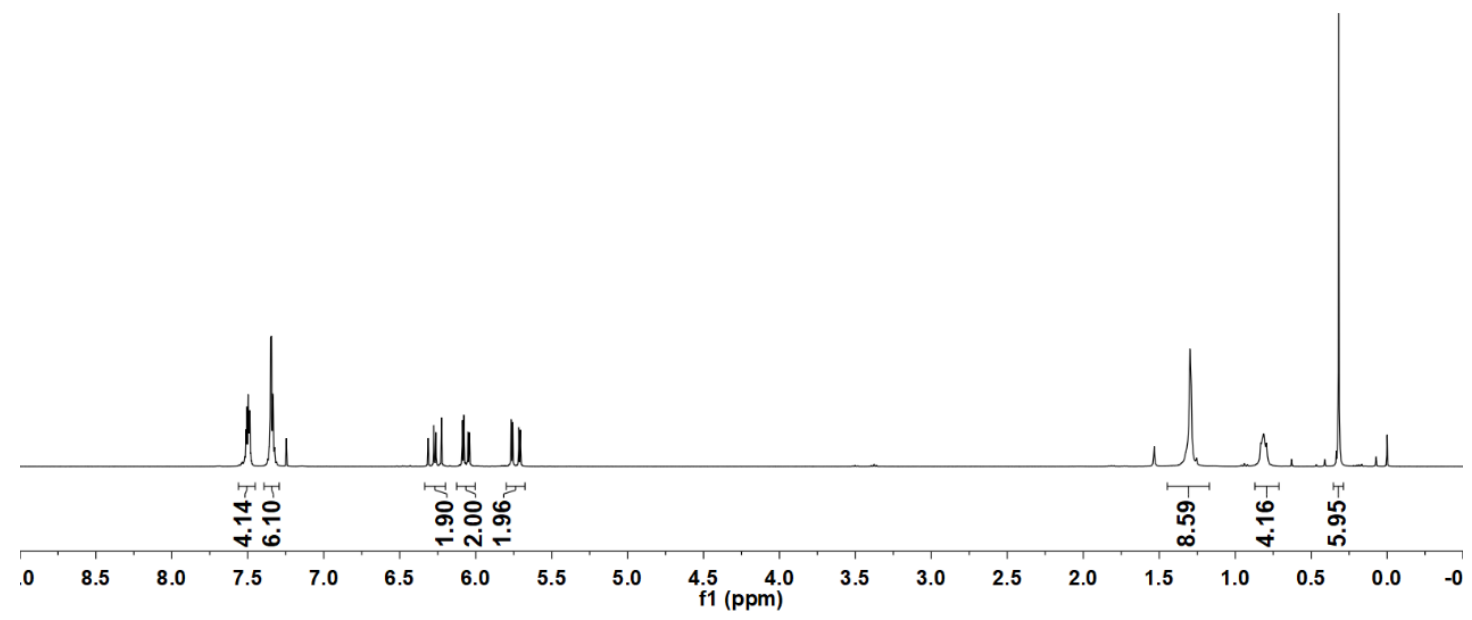

番

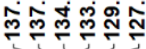

gำ웅

视西

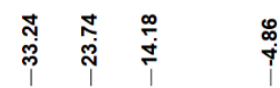
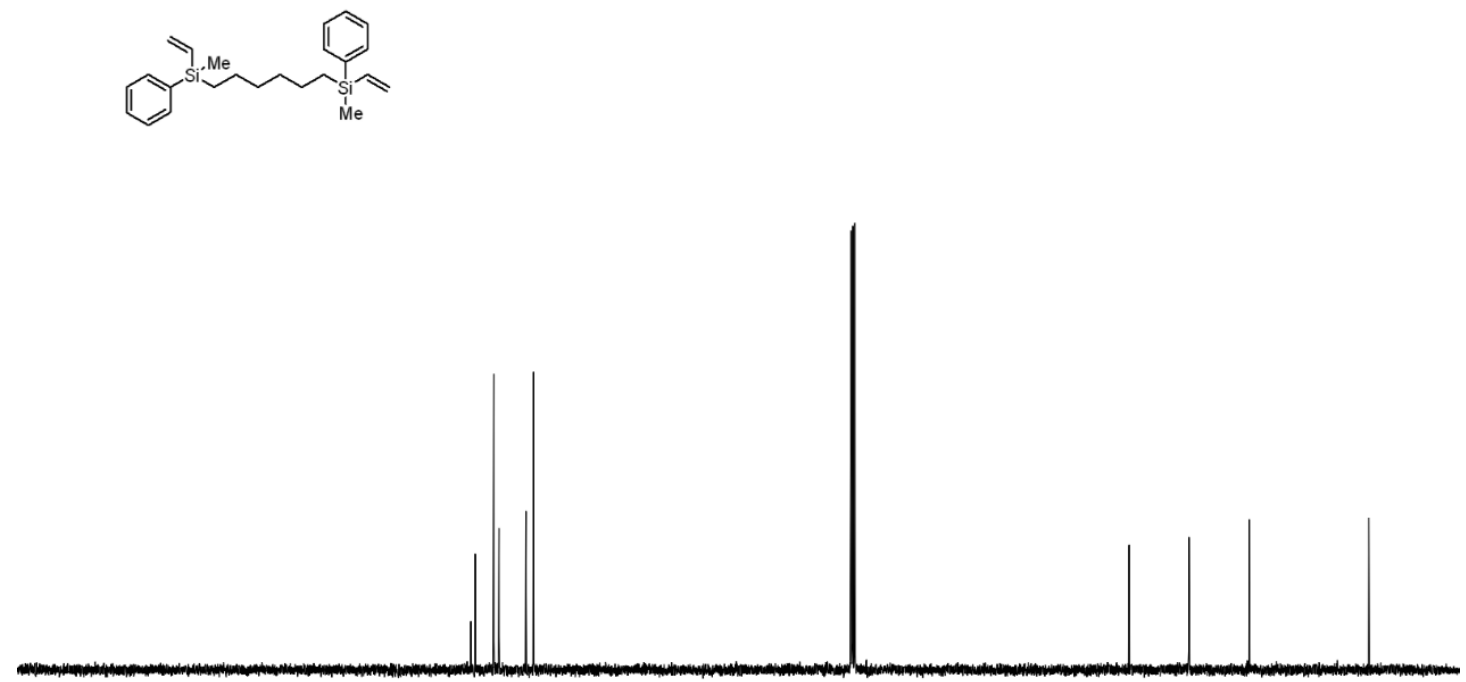

$\begin{array}{llllllllllllllllllllll}10 & 200 & 190 & 180 & 170 & 160 & 150 & 140 & 130 & 120 & 110 & \begin{array}{l}100 \\ \mathrm{f} 1(\mathrm{ppm})\end{array} & 80 & 70 & 60 & 50 & 40 & 30 & 20 & 10 & 0 & -10\end{array}$ 
3w; ${ }^{29} \mathrm{Si}$ NMR (79 $\left.\mathrm{MHz}, \mathrm{CDCl}_{3}\right)$

$\stackrel{\check{0}}{i}$

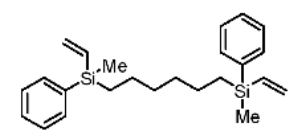

$\underbrace{}_{50}$ 
3x; ${ }^{1} \mathrm{H}$ NMR (400 MHz, CDCl 3$\left.) ;{ }^{13} \mathrm{C} \mathrm{NMR} \mathrm{(100} \mathrm{MHz,} \mathrm{CDCl}_{3}\right)$

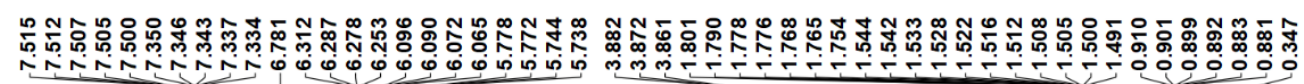
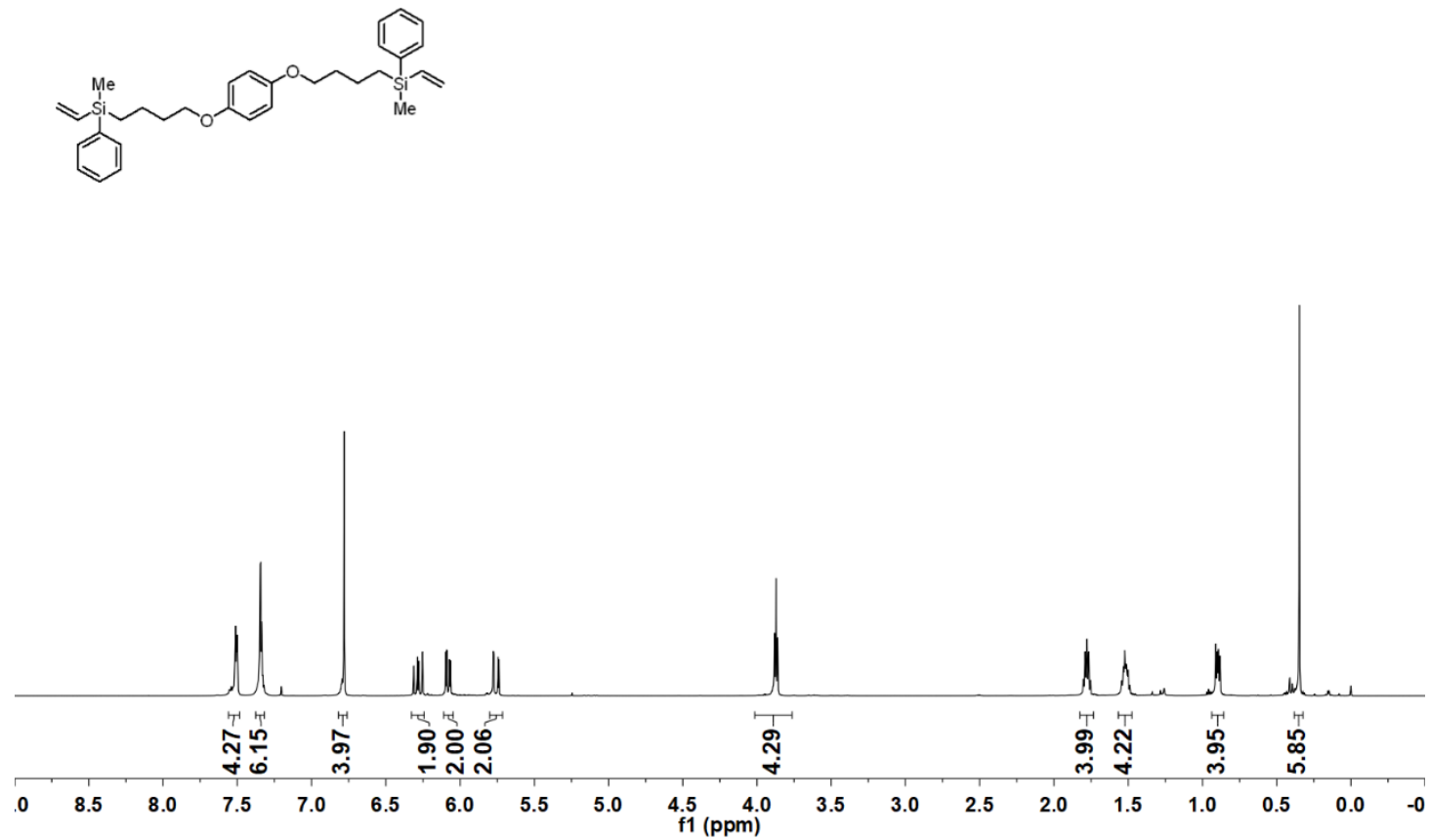

常
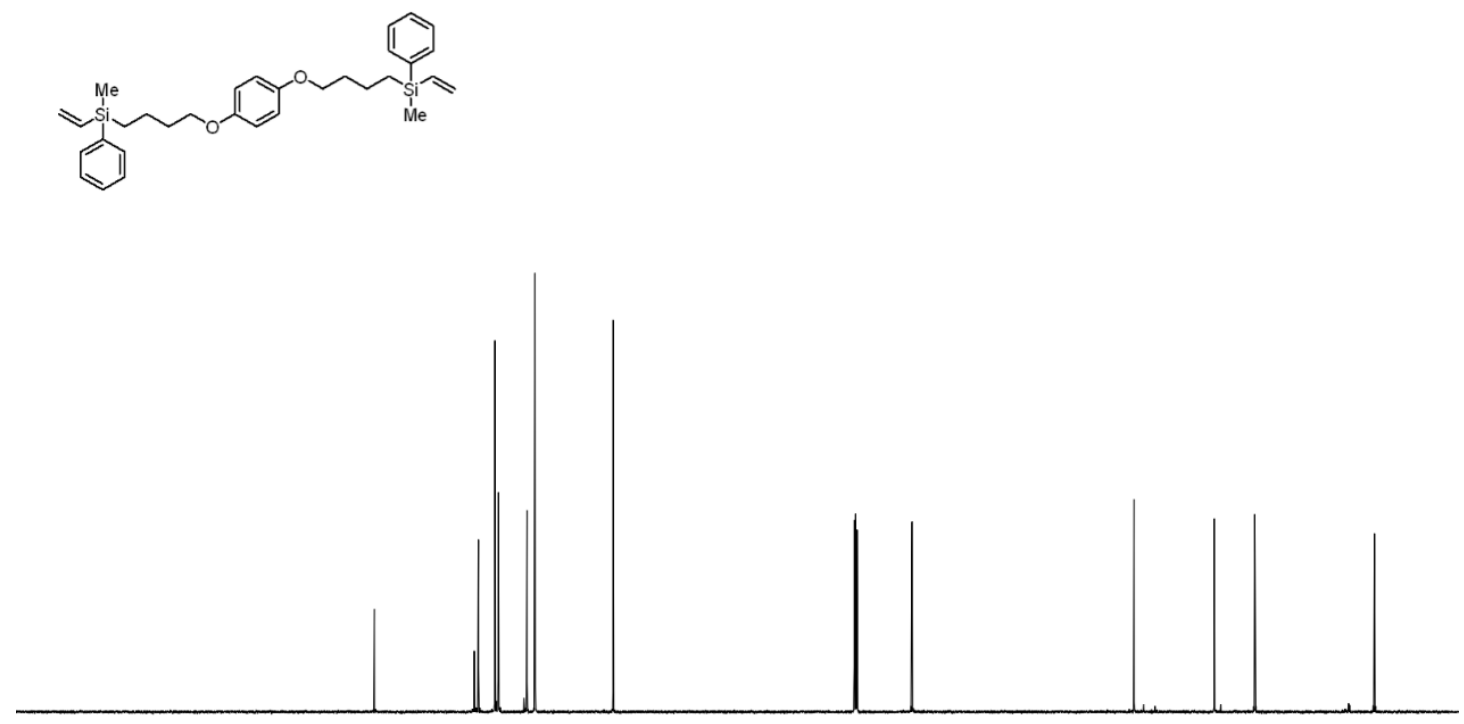

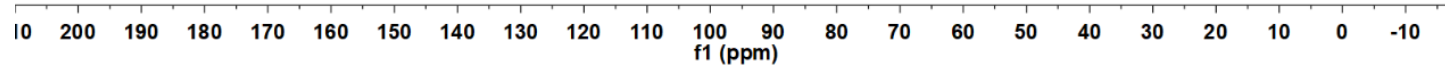


3x; $\left.{ }^{29} \mathrm{Si} \mathrm{NMR} \mathrm{(79} \mathrm{MHz,} \mathrm{CDCl}_{3}\right)$

กึ่

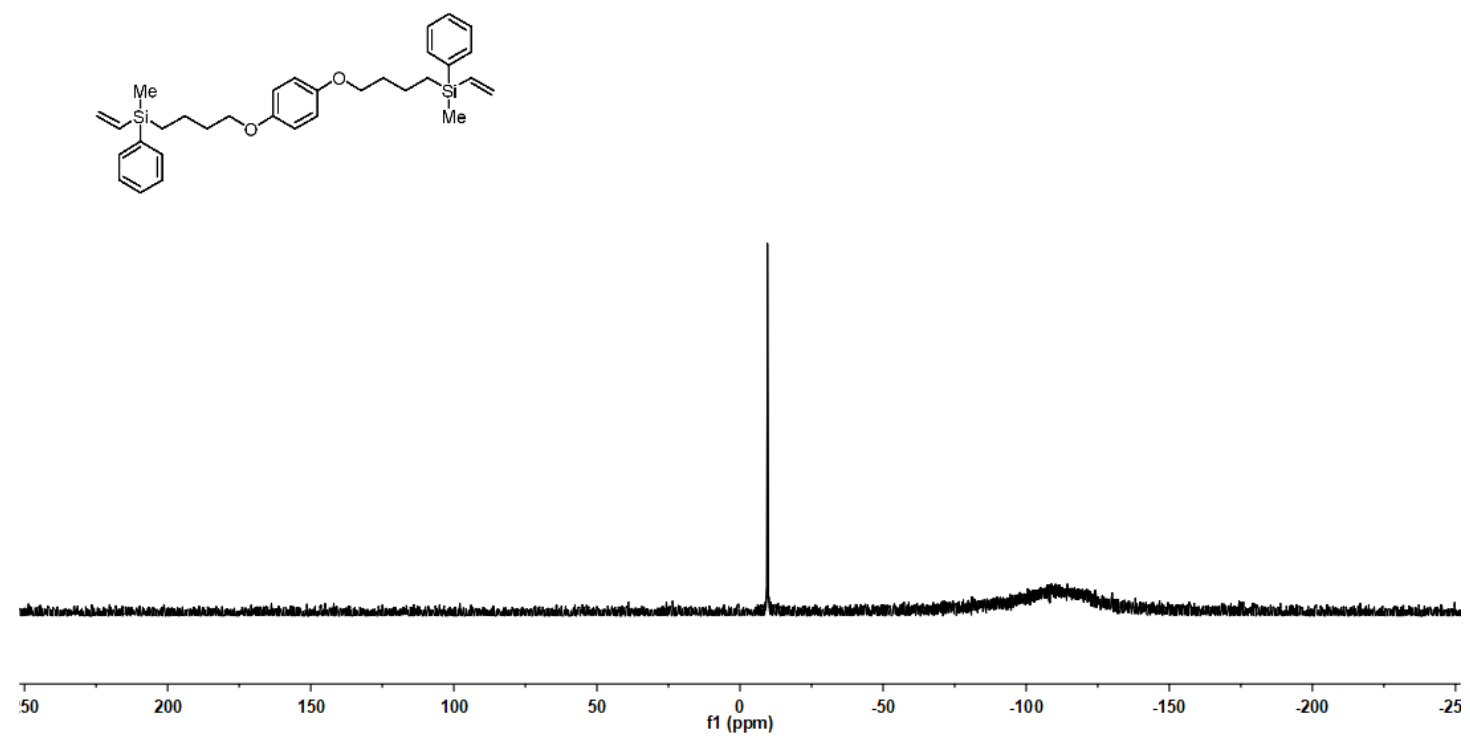


3y; ${ }^{1} \mathrm{H}$ NMR (400 MHz, CDCl $\left.) ;{ }^{13} \mathrm{C} \mathrm{NMR} \mathrm{(100} \mathrm{MHz,} \mathrm{CDCl} 3\right)$

Pros?

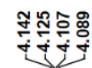

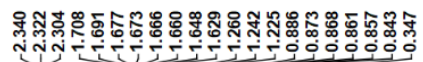

(c)

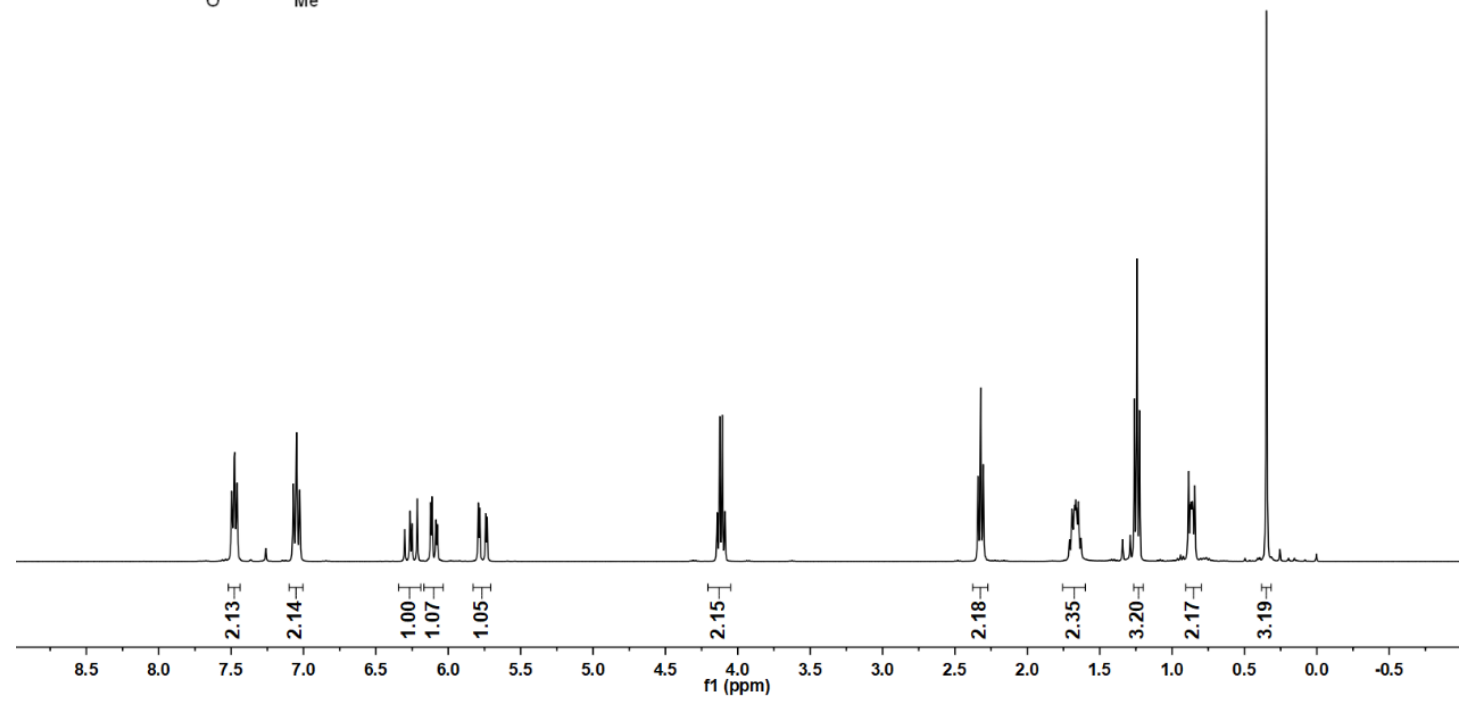

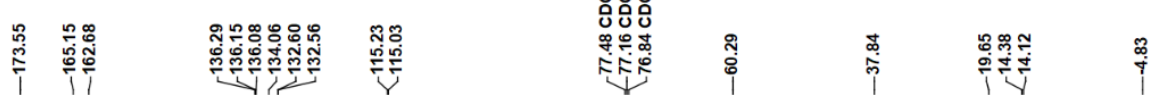

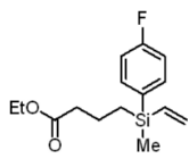

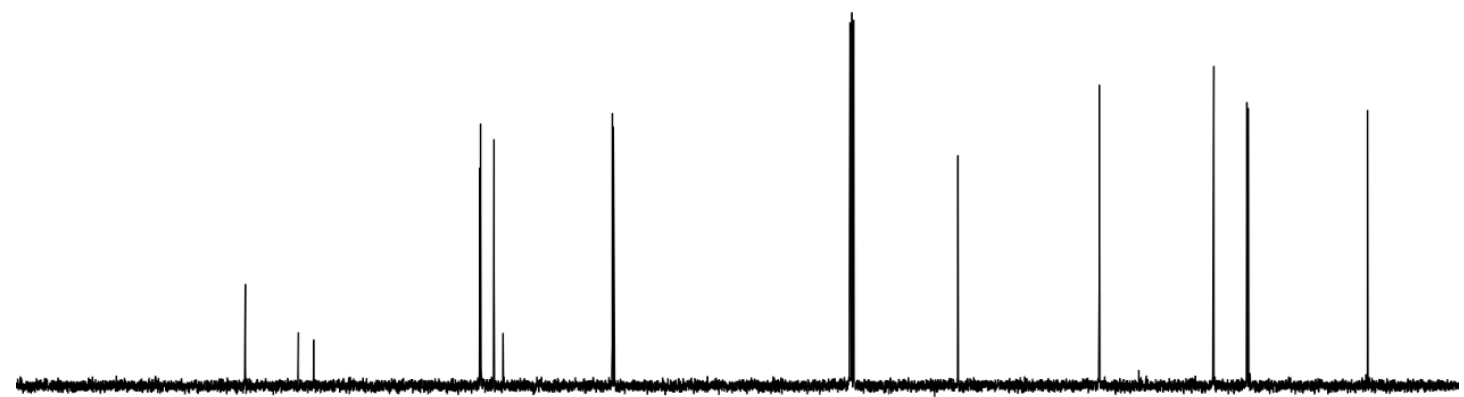

\begin{tabular}{llllllllllll}
\hline 10 & 200 & 190 & 180 & 170 & 160 & 150 & 140 & 130 & 120 & 110 & $\underset{f 1}{100} 90$ \\
$\mathrm{f} 1(\mathrm{ppm})$
\end{tabular}

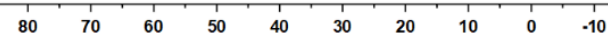


3y; ${ }^{19}$ F NMR (376 $\left.\mathrm{MHz} \mathrm{CDCl}_{3}\right) ;{ }^{29} \mathrm{Si}$ NMR (79 $\left.\mathrm{MHz}, \mathrm{CDCl}_{3}\right)$

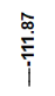
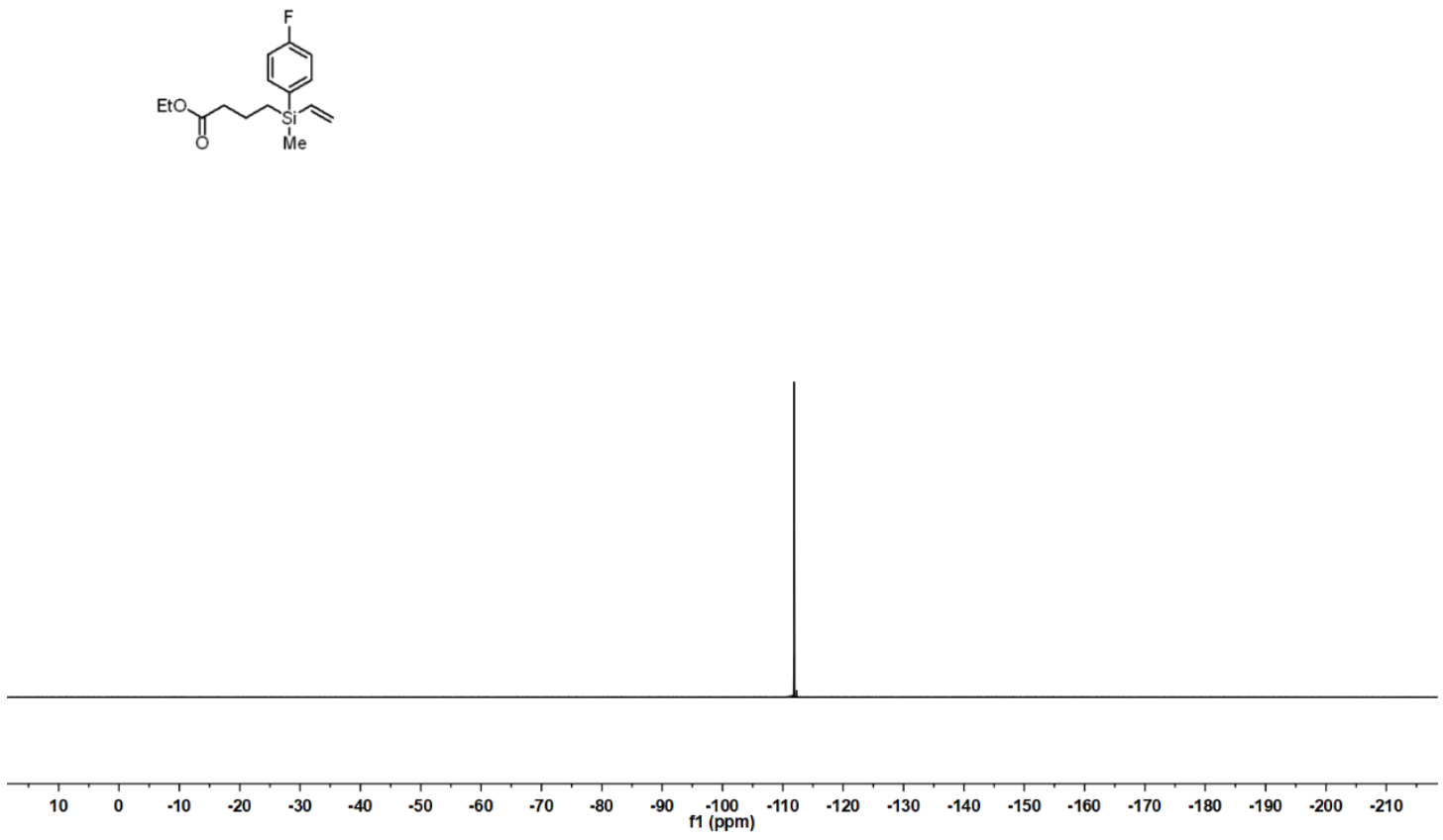

$\stackrel{\vec{m}}{i} \underset{i}{i}$
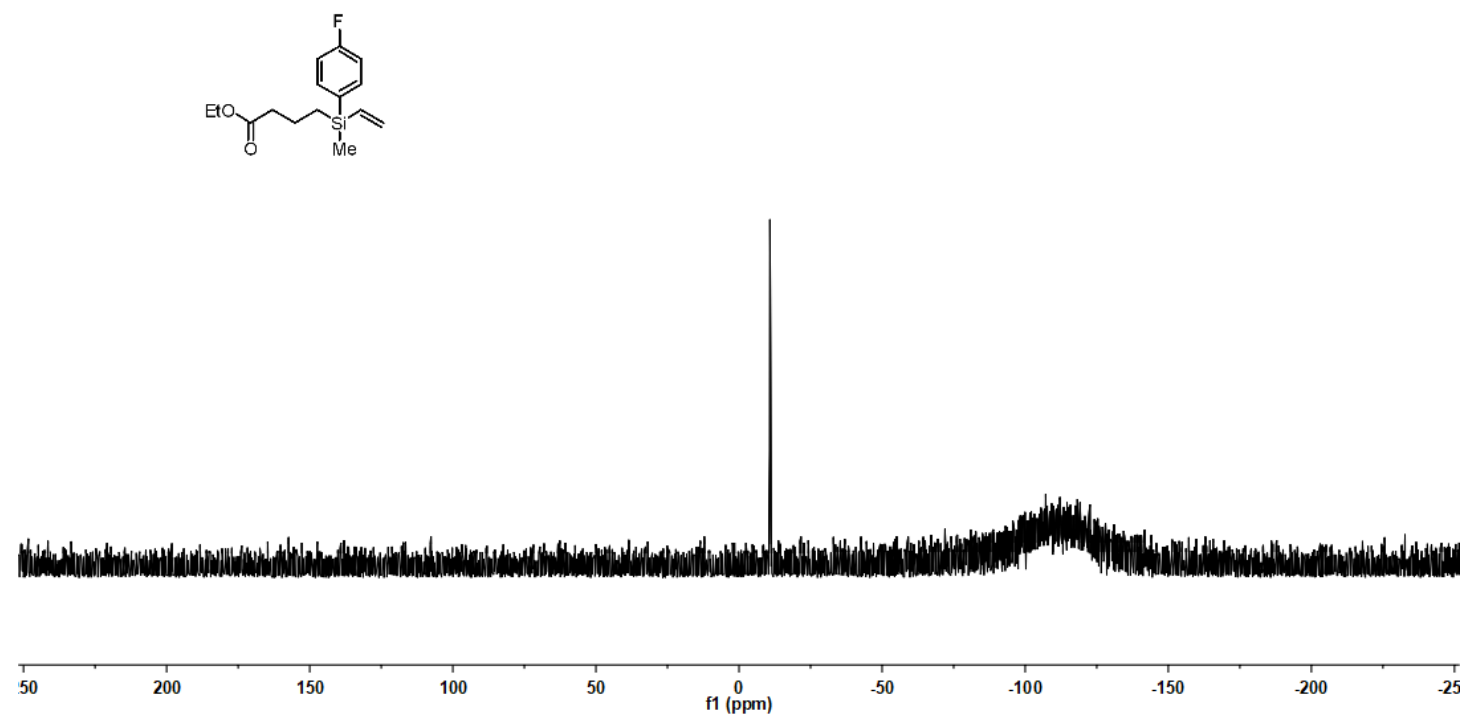

S82 
3z; ${ }^{1} \mathrm{H}$ NMR (400 MHz, CDCl 3$\left.) ;{ }^{13} \mathrm{C} \mathrm{NMR} \mathrm{(100} \mathrm{MHz,} \mathrm{CDCl} 3\right)$

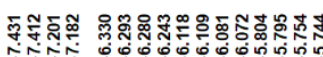

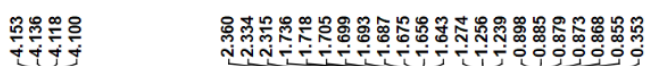

$\underbrace{M e}_{\substack{\text { si } \\ \text { Me }}}$

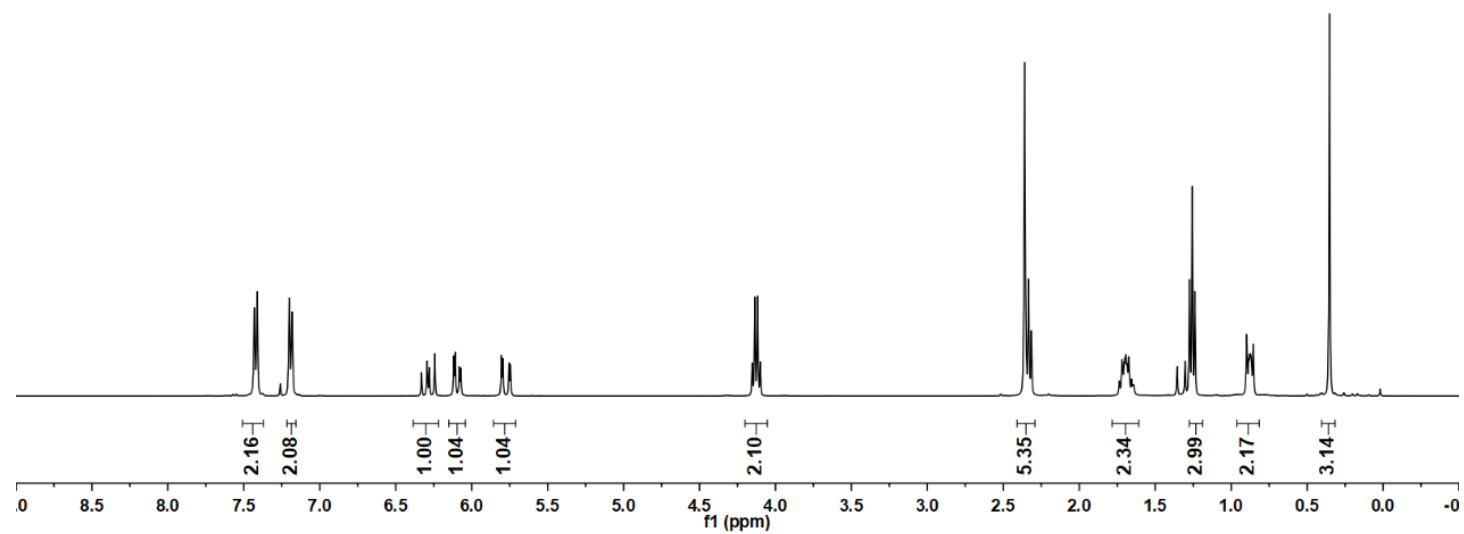

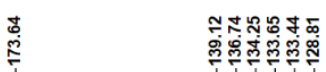

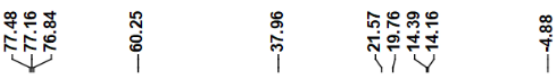

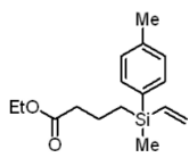

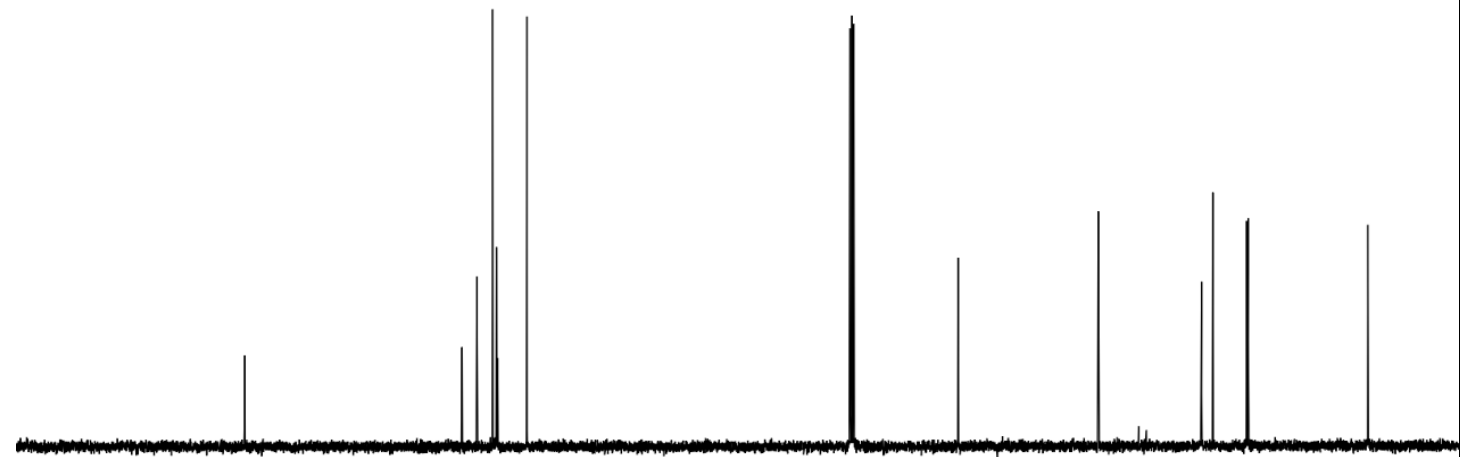

$\begin{array}{lllllllllllll}10 & 200 & 190 & 180 & 170 & 160 & 150 & 140 & 130 & 120 & 110 & 100 & 90 \\ \mathrm{f} 1(\mathrm{ppm})\end{array}$ 
3z; ${ }^{29} \mathrm{Si}$ NMR (79 MHz, CDCl 3$)$

ঙ̊ำ<smiles>[3H][Si]([3H])([3H])[3H]</smiles>

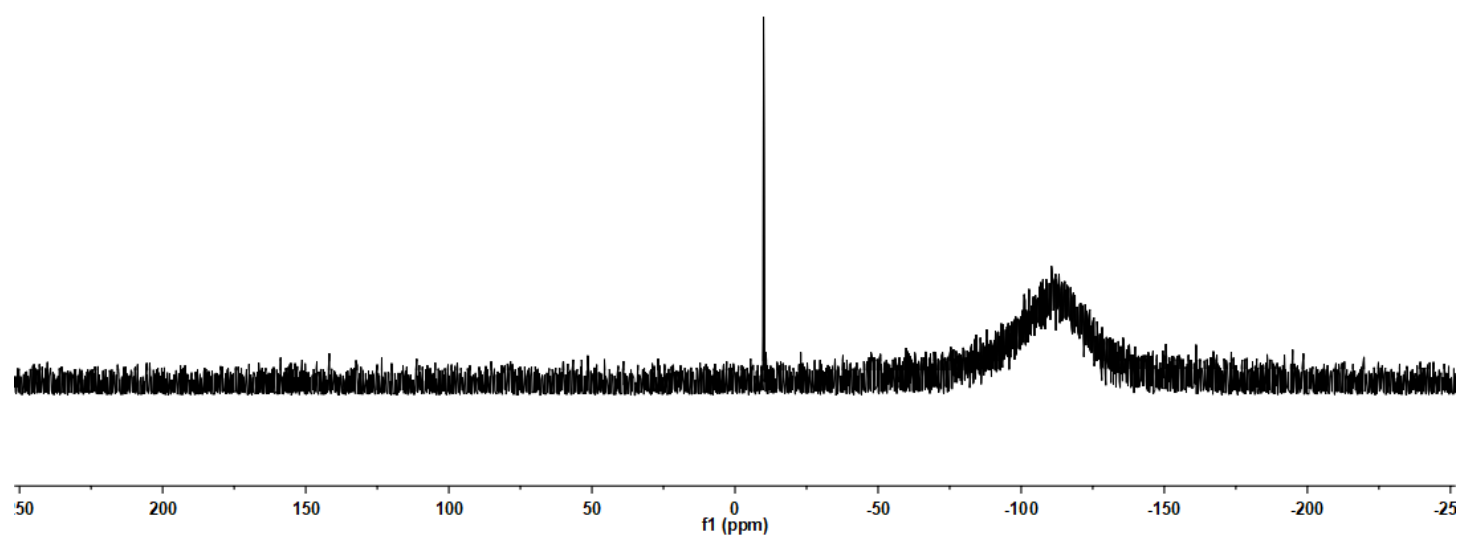


3aa; ${ }^{1} \mathrm{H}$ NMR (400 MHz, CDCl 3$) ;{ }^{13} \mathrm{C}$ NMR (100 MHz, CDCl $)$

草受

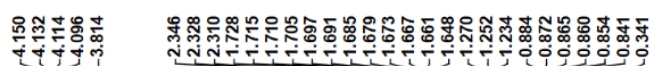

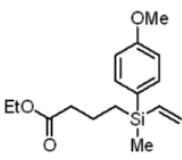

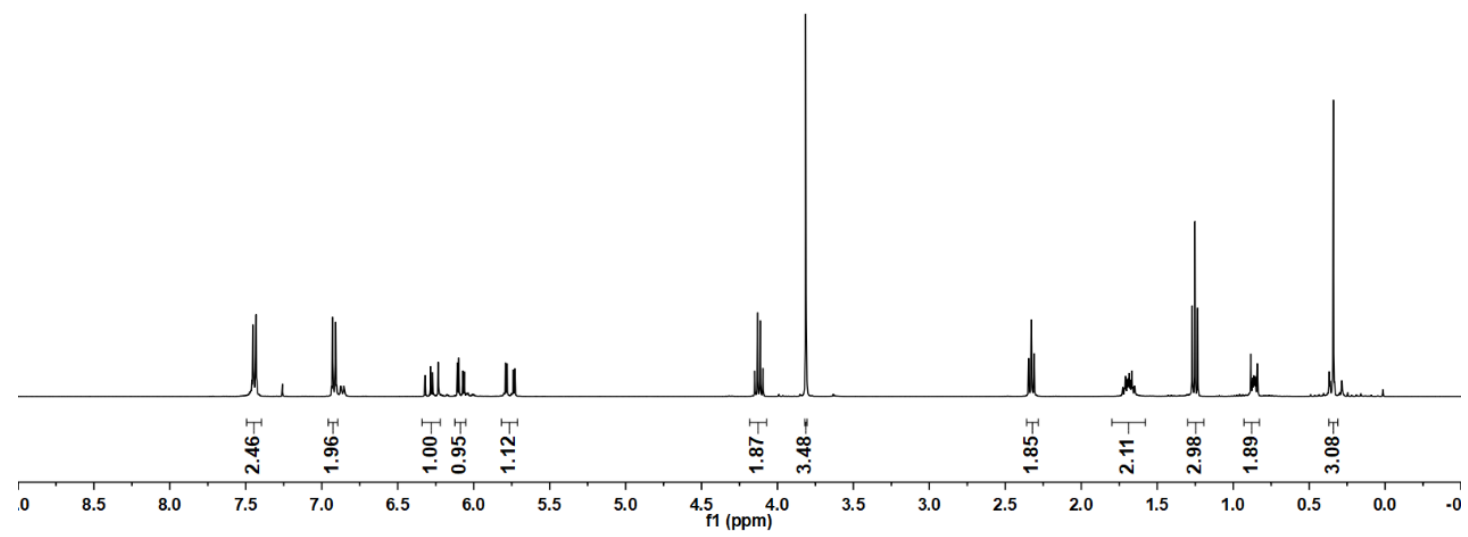

皇

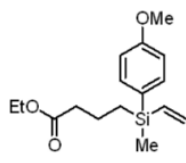

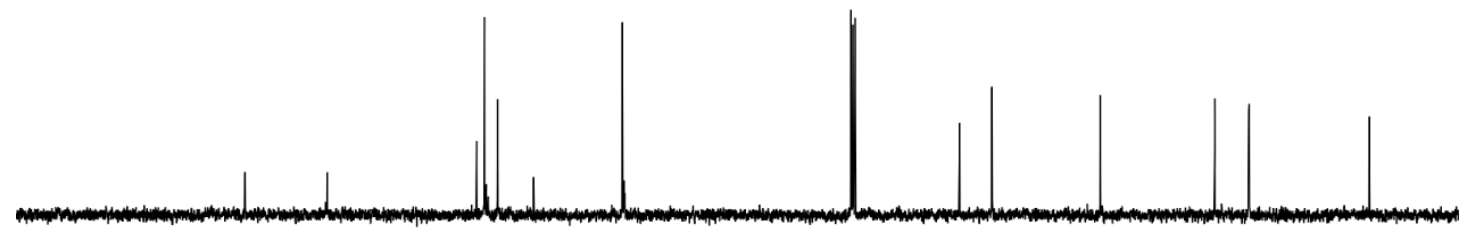

\begin{tabular}{llllllllllll}
\hline 10 & 200 & 190 & 180 & 170 & 160 & 150 & 140 & 130 & 120 & 110 & $\underset{100}{100}(\mathrm{ppm})$
\end{tabular} 
3aa; ${ }^{29} \mathrm{Si}$ NMR (79 MHz, CDCl 3$)$

$\stackrel{\stackrel{m}{\rightleftarrows}}{i}$

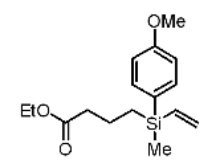

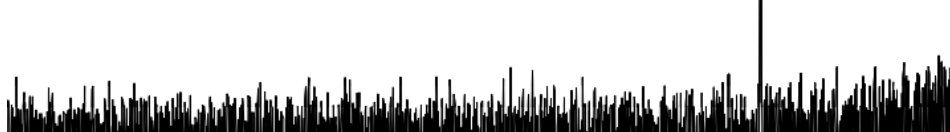

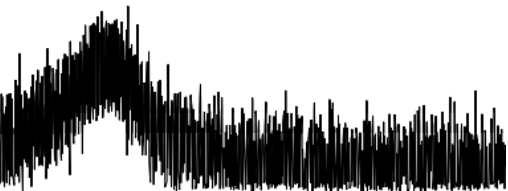

50

150

100

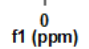

$-50$

$-100$

$-150$

$-200$ . 
3ab; ${ }^{1} \mathrm{H}$ NMR (400 MHz, $\left.\mathrm{CDCl}_{3}\right) ;{ }^{13} \mathrm{C}$ NMR (100 $\left.\mathrm{MHz}, \mathrm{CDCl}_{3}\right)$
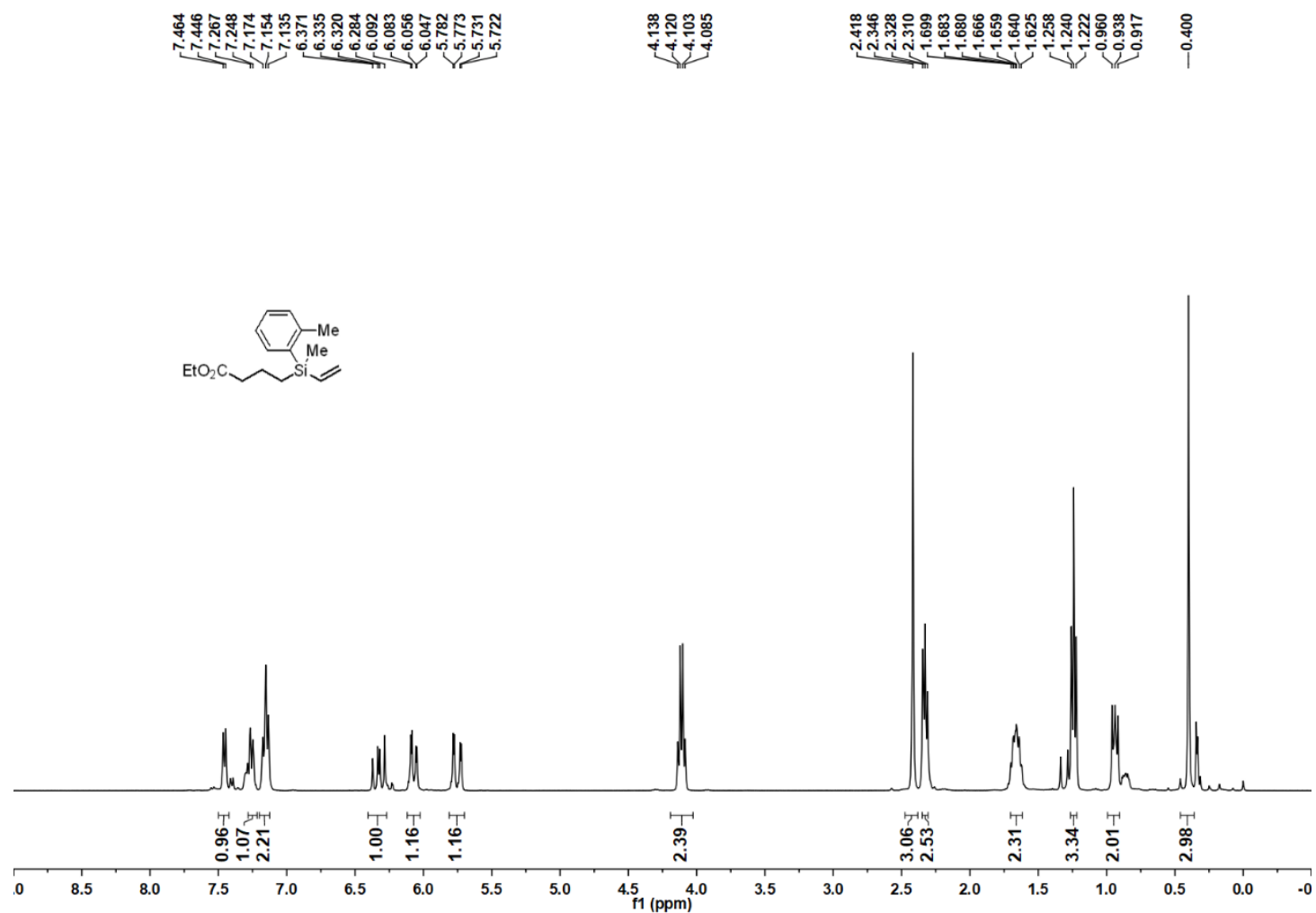

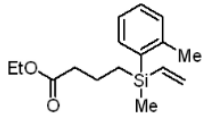

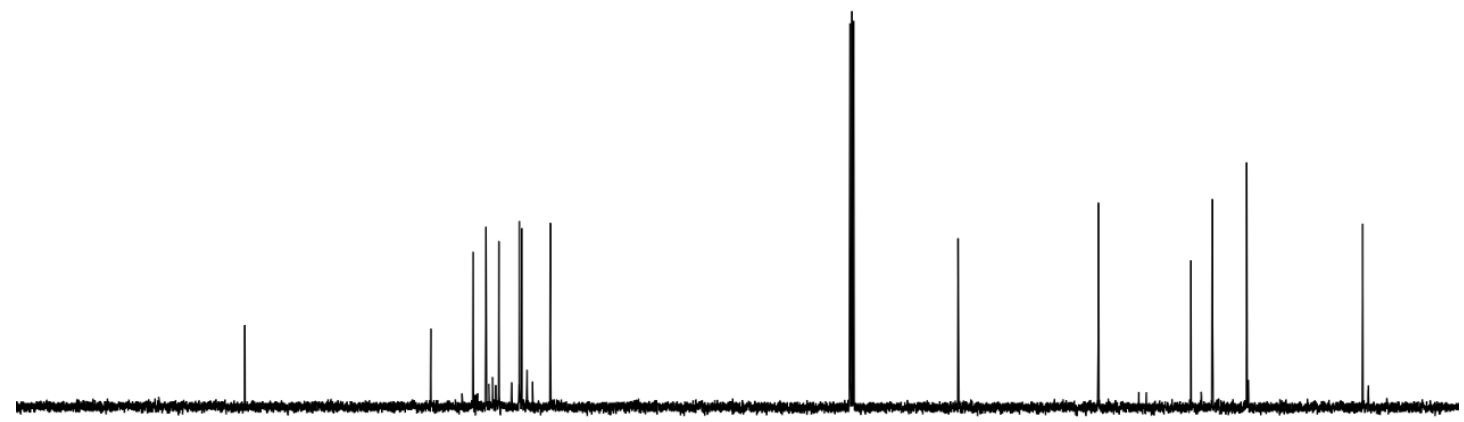

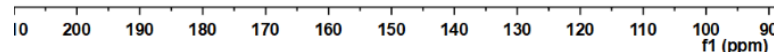


3ab; ${ }^{29} \mathrm{Si}$ NMR (79 $\left.\mathrm{MHz}, \mathrm{CDCl}_{3}\right)$

$\stackrel{8}{i}$

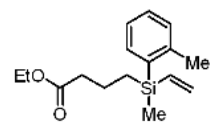

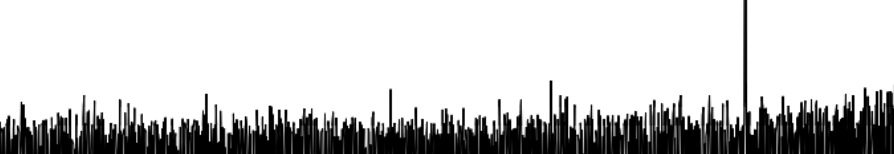

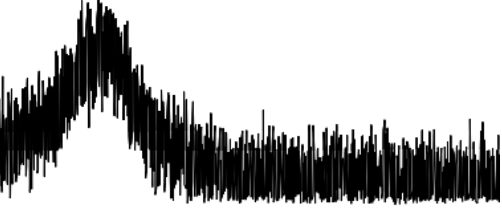

50

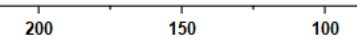

0
f1 $(\mathrm{ppm})$

$-50$

$-100$

$-150$

$-200$ 
3ad; ${ }^{1} \mathrm{H}$ NMR (400 MHz, CDCl 3$) ;{ }^{13} \mathrm{C}$ NMR (100 MHz, $\left.\mathrm{CDCl}_{3}\right)$

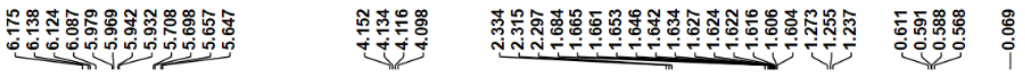

$\mathrm{EtO}_{2} \mathrm{C} \sim \overbrace{}^{\mathrm{Me}} \mathrm{Me}$

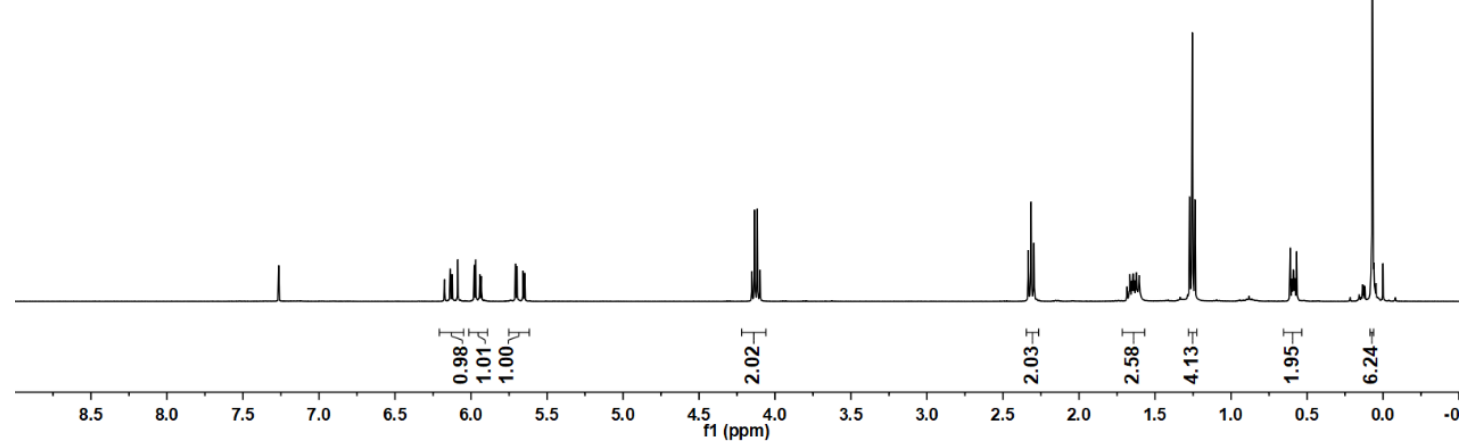

荥

$\mathrm{EtO}_{2} \mathrm{C} \sim \sim_{\mathrm{Me}}^{\mathrm{Me}}=$

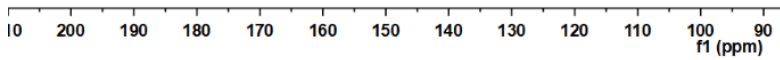


3ad; ${ }^{29} \mathrm{Si}$ NMR (79 $\left.\mathrm{MHz}, \mathrm{CDCl}_{3}\right)$

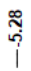

$\mathrm{EtO}_{2} \mathrm{C}$ M $\mathrm{Me}_{\mathrm{Si}} \mathrm{Me}$

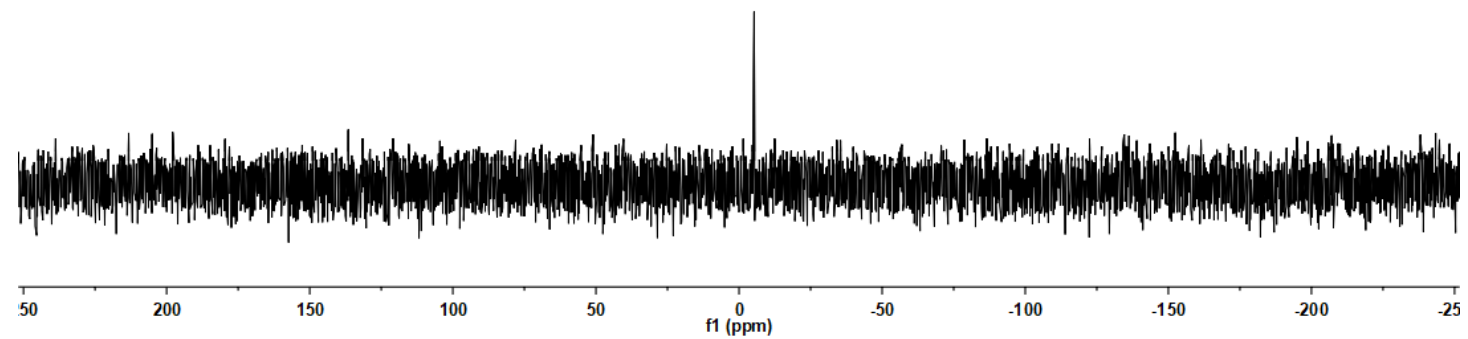


3ae; ${ }^{1} \mathrm{H}$ NMR (400 MHz, CDCl $) ;{ }^{13} \mathrm{C}$ NMR (100 MHz, CDCl $)$

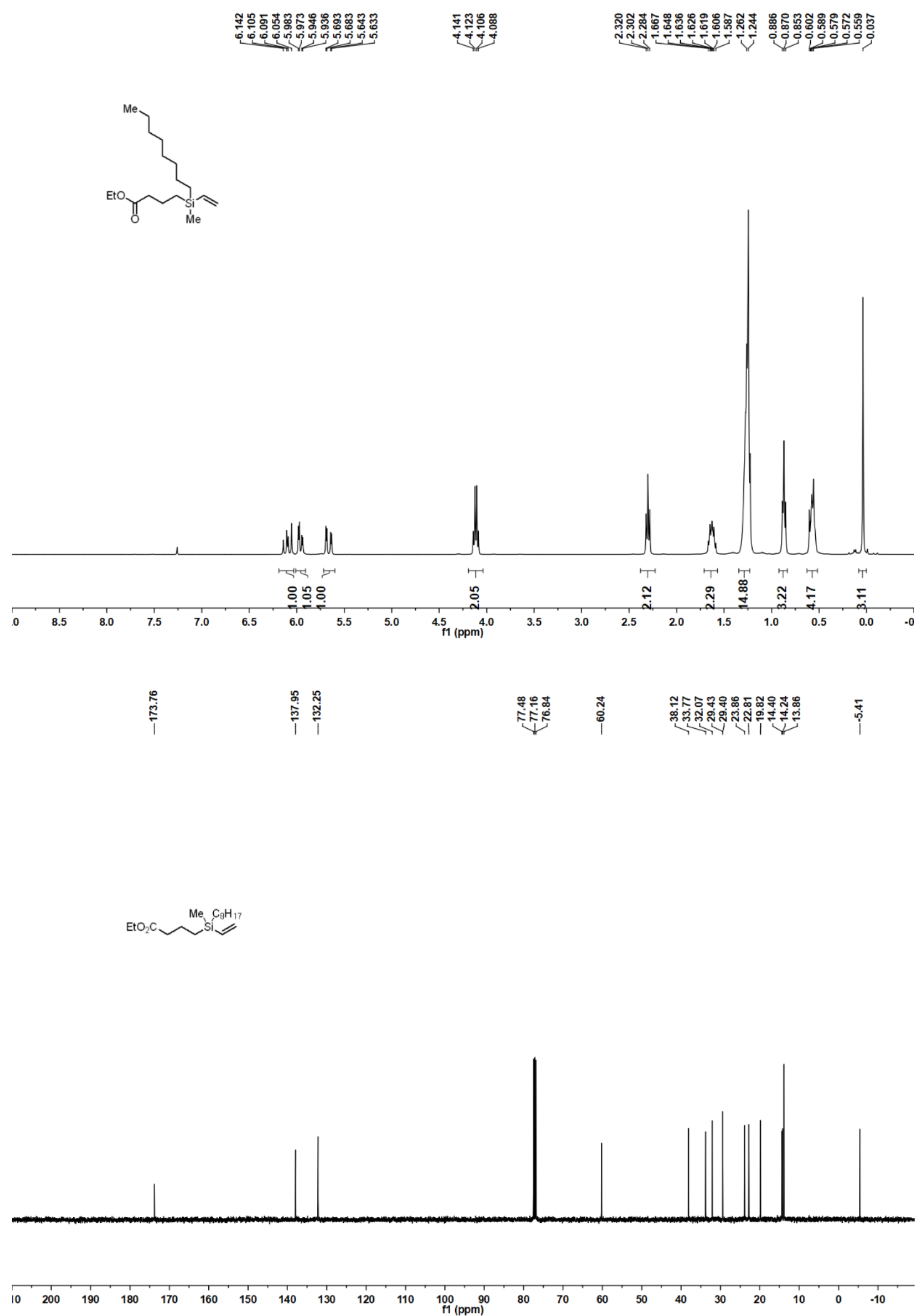




\section{3ae; ${ }^{29} \mathrm{Si}$ NMR (79 MHz, CDCl 3$)$}
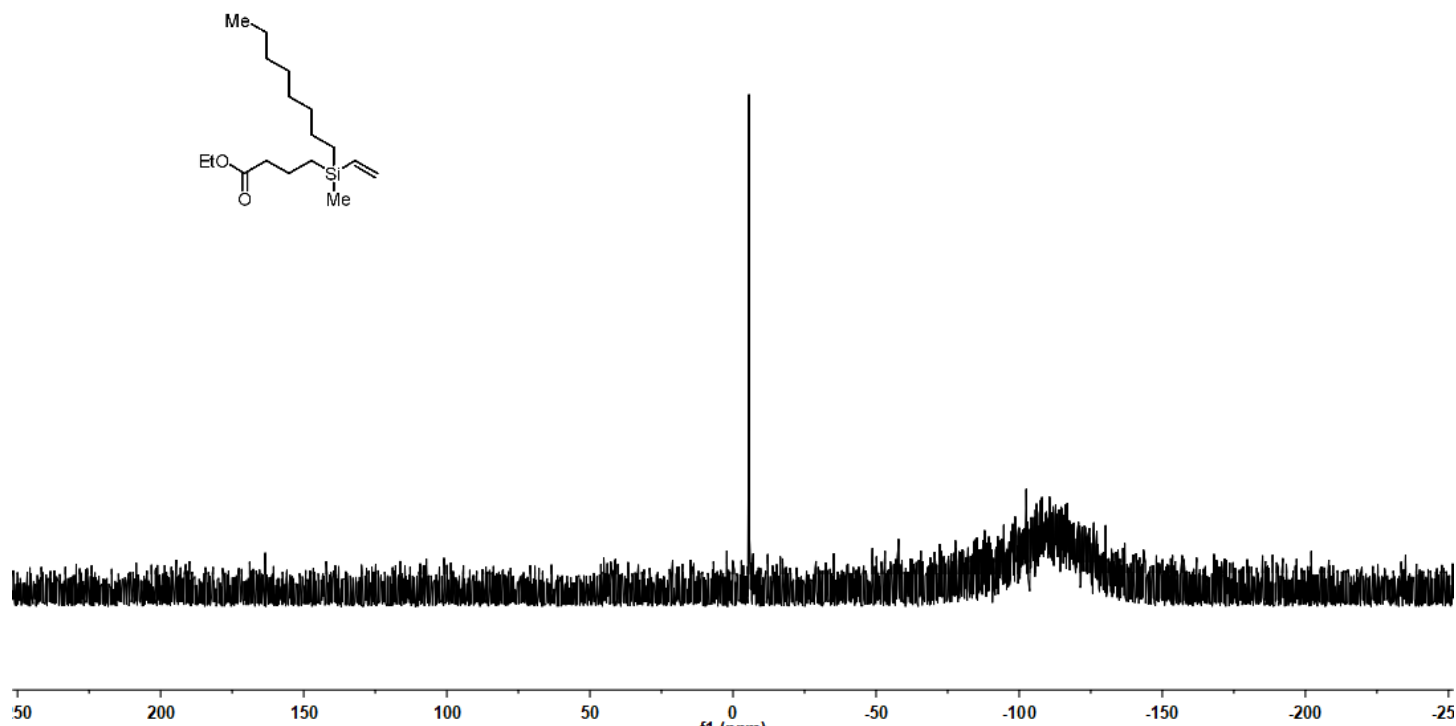

150

100

$\stackrel{0}{\mathrm{f} 1(\mathrm{ppm})}$

$-50$

$-100$

$-150$

$-200$ 
3af; ${ }^{1} \mathrm{H}$ NMR (400 MHz, CDCl $) ;{ }^{13} \mathrm{C}$ NMR (100 MHz, CDCl $)$

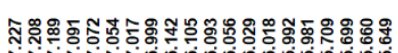

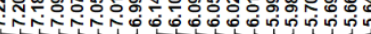

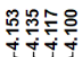

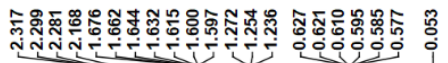

$\overbrace{\substack{\text { sis } \\ \text { me }}}^{\text {Eto }}$

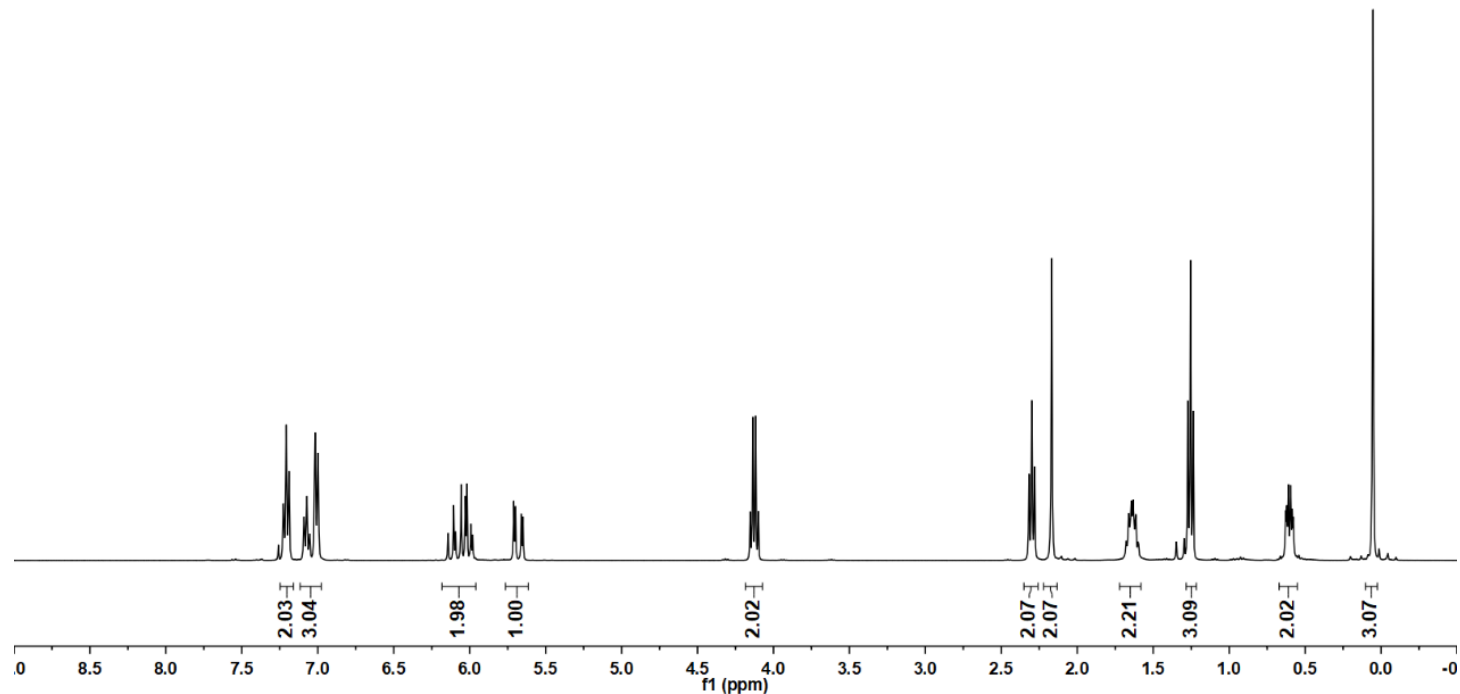

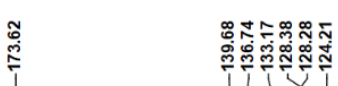

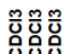

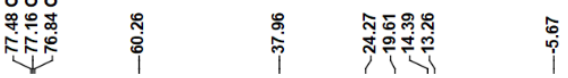

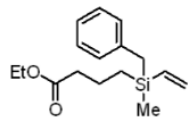

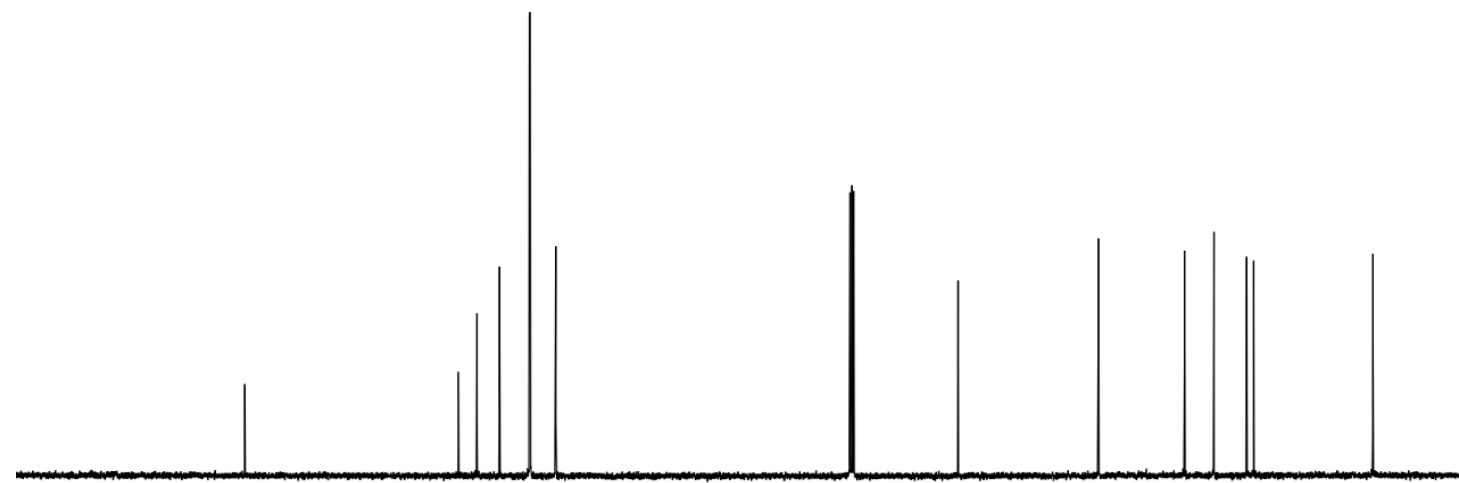

\begin{tabular}{|llllllllllllllllllllllllllllllllll}
\hline 10 & 200 & 190 & 180 & 170 & 160 & 150 & 140 & 130 & 120 & 110 & 100 & 90 & 80 & 70 & 60 & 50 & 40 & 30 & 20 & 10 & 0 & -10 & 1
\end{tabular} 
3af; ${ }^{29} \mathrm{Si}$ NMR (79 MHz, $\left.\mathrm{CDCl}_{3}\right)$

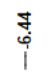
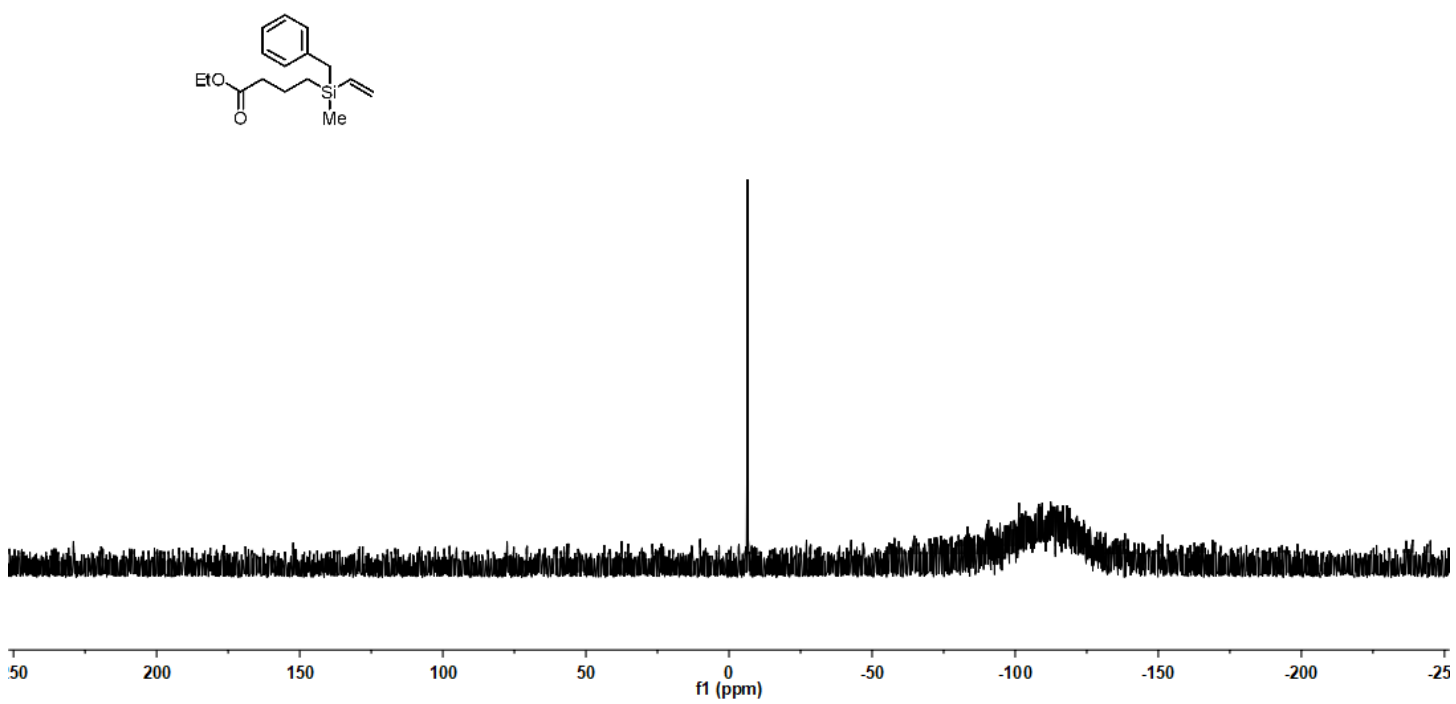

S94 
3ag; ${ }^{1} \mathrm{H}$ NMR (400 MHz, CDCl 3$) ;{ }^{13} \mathrm{C}$ NMR (100 MHz, CDCl$)$

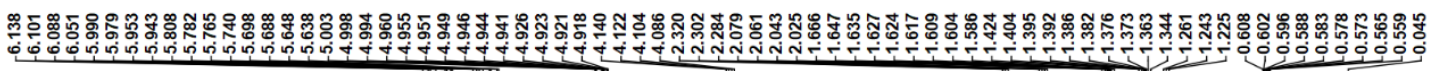
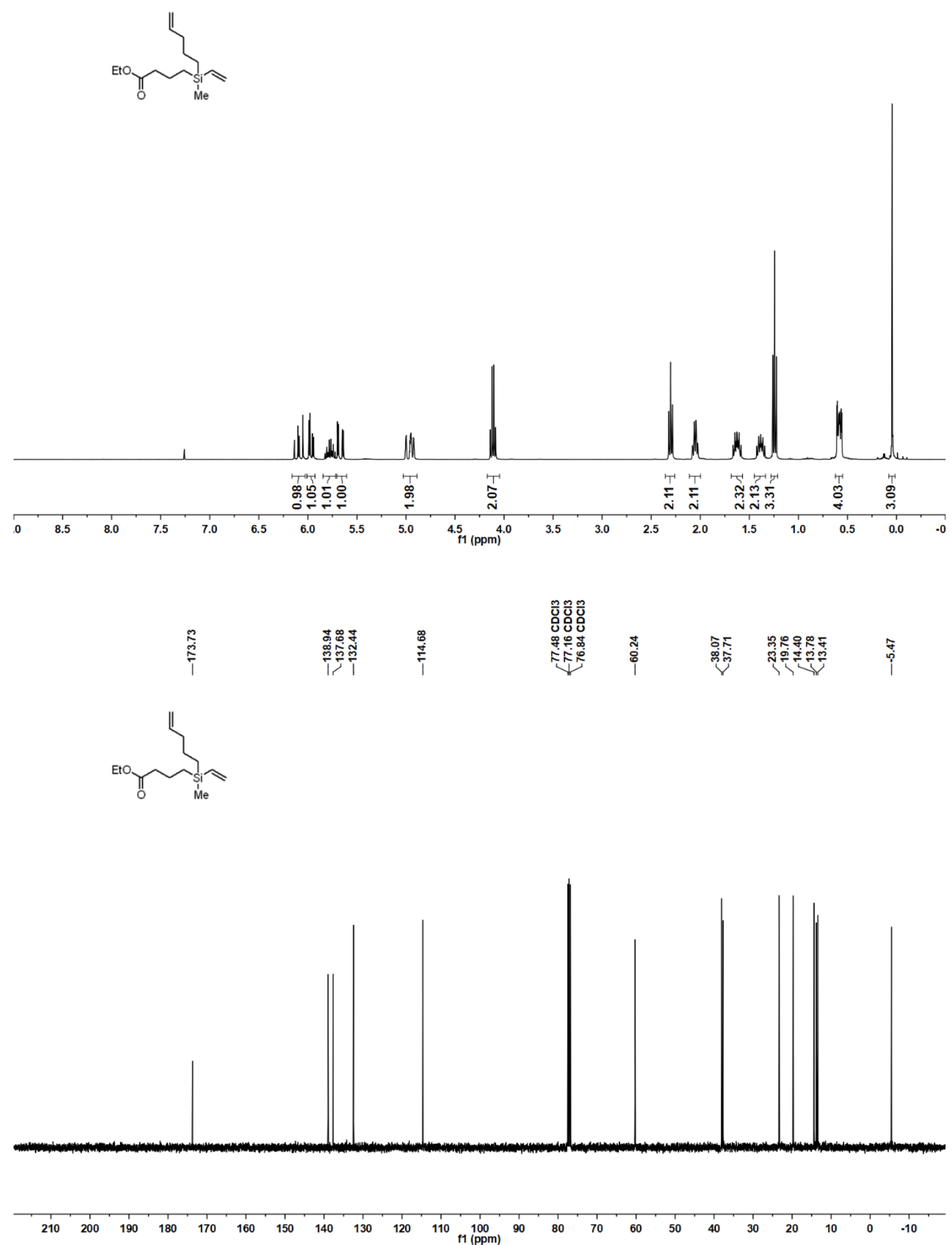
3ag; ${ }^{29} \mathrm{Si}$ NMR (79 MHz, $\left.\mathrm{CDCl}_{3}\right)$

$$
\text { क̊. }
$$

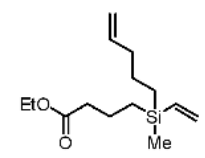

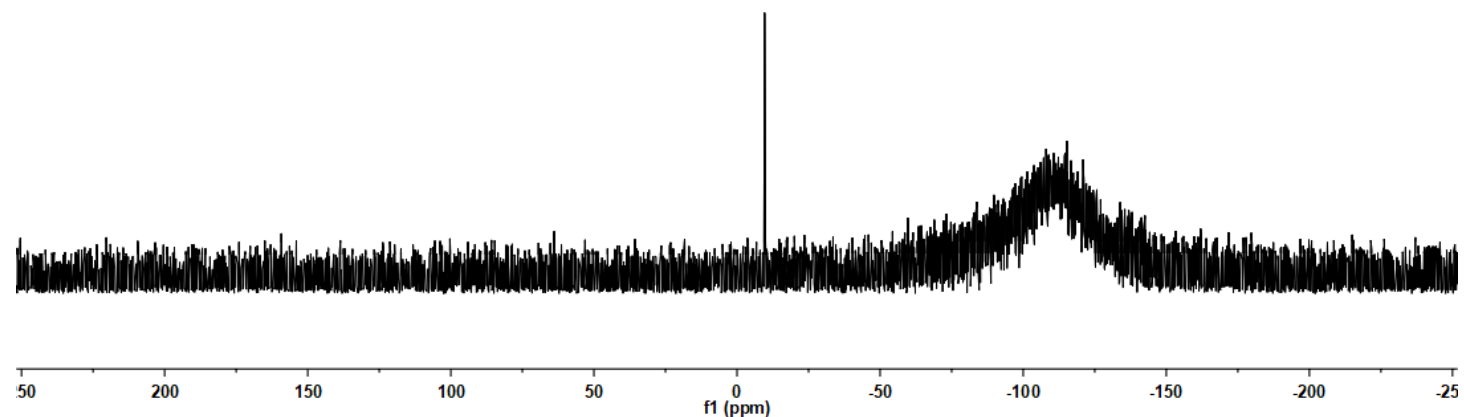


3ah; ${ }^{1} \mathrm{H}$ NMR (400 MHz, CDCl 3$) ;{ }^{13} \mathrm{C}$ NMR (100 $\mathrm{MHz}^{\mathrm{CDCl}}$ )

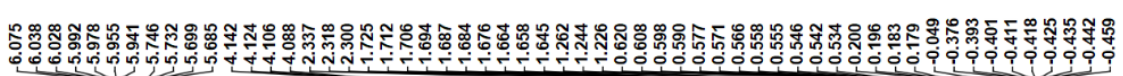

$\overbrace{\substack{\text { Sil } \\ \text { ine }}}^{\text {Eto }}$

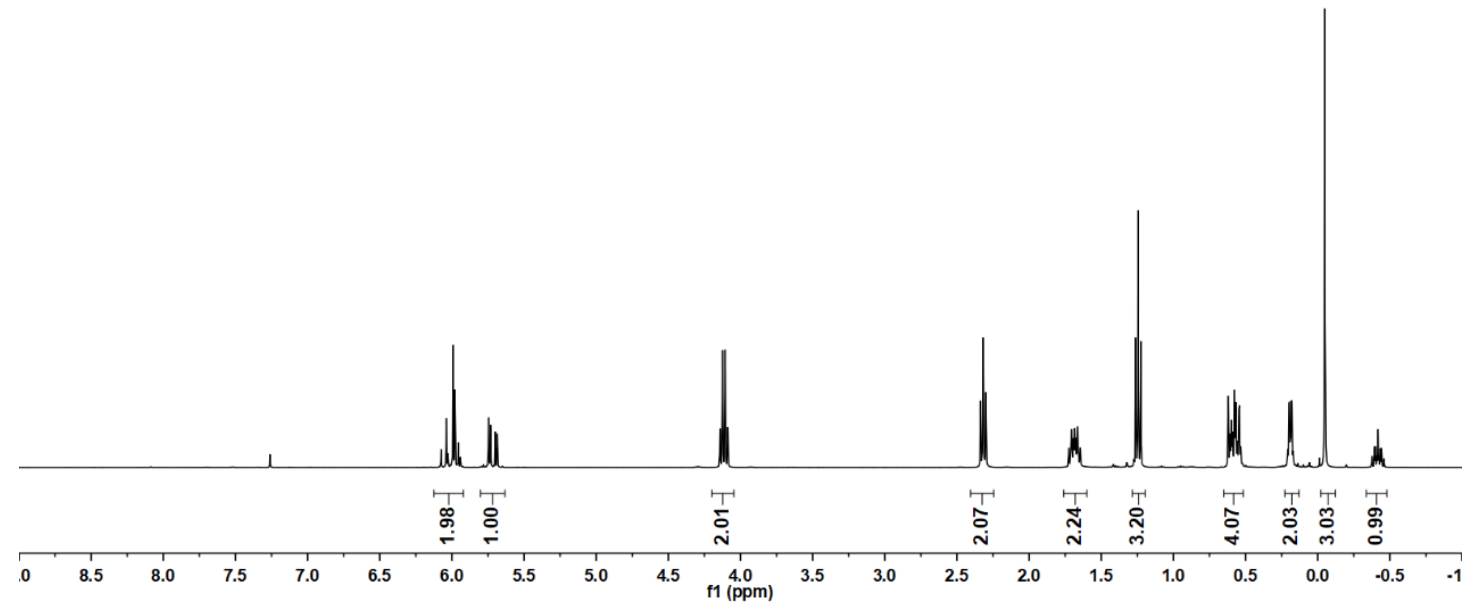

要

$\operatorname{lom}_{\substack{\text { is } \\ \text { Me }}}$

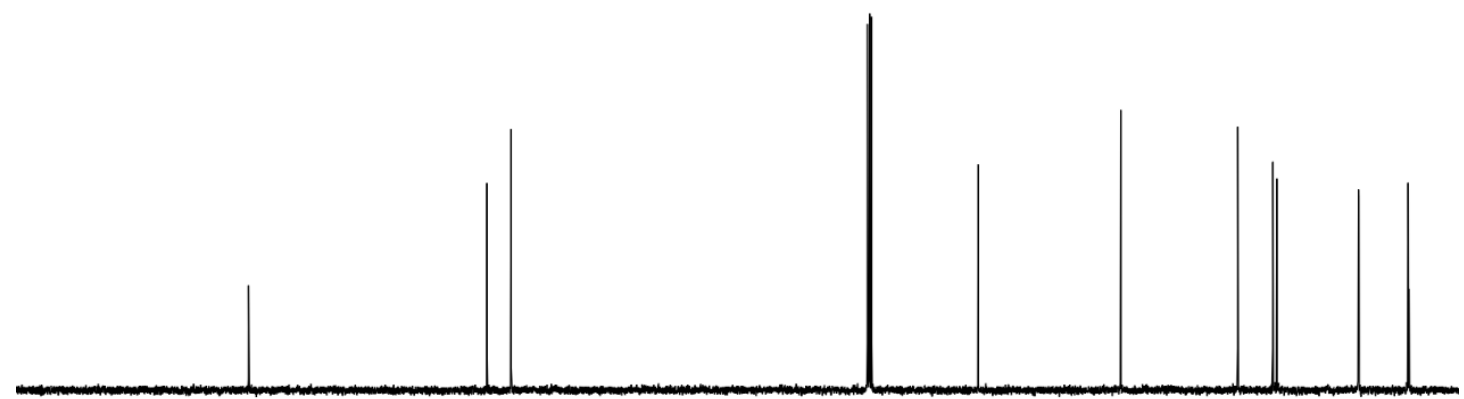

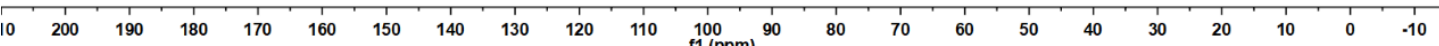


3ah; ${ }^{29} \mathrm{Si}$ NMR (79 MHz, CDCl 3$)$

\ุ

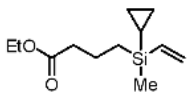

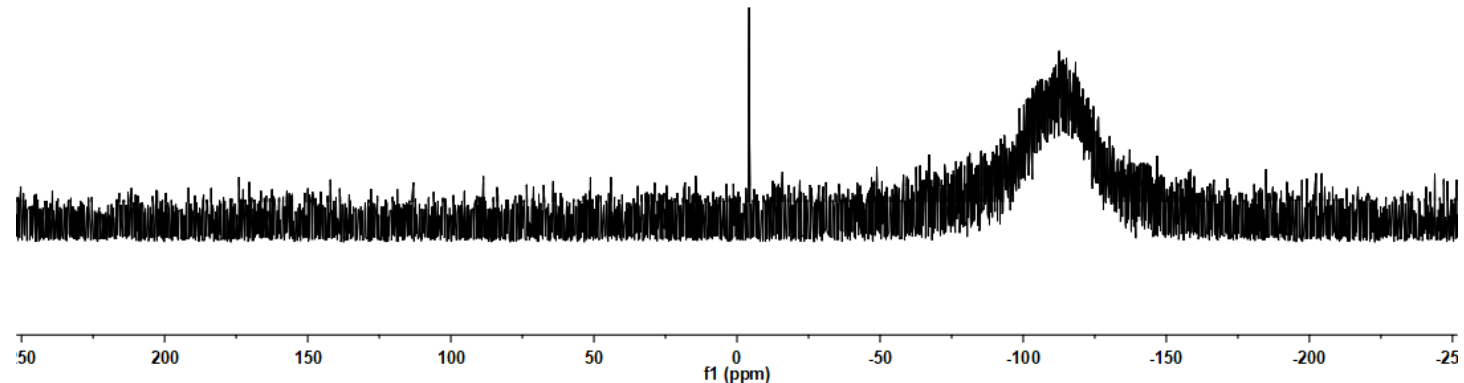


3ai; ${ }^{1} \mathrm{H}$ NMR (400 MHz, CDCl $) ;{ }^{13} \mathrm{C}$ NMR (100 MHz, CDCl 3$)$

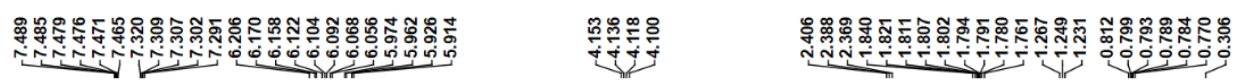

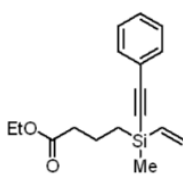

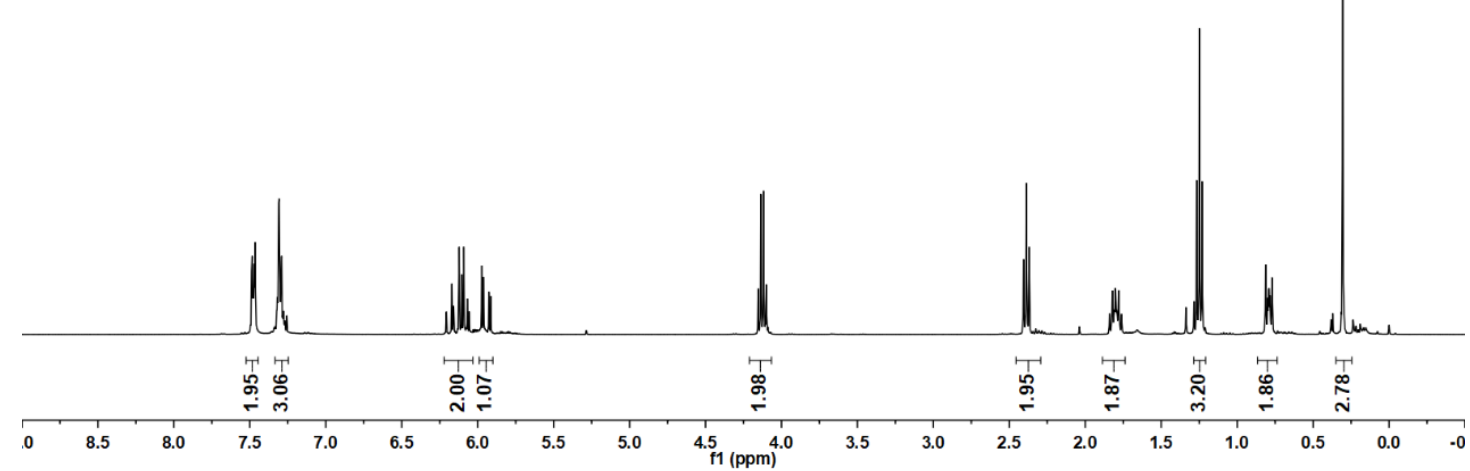

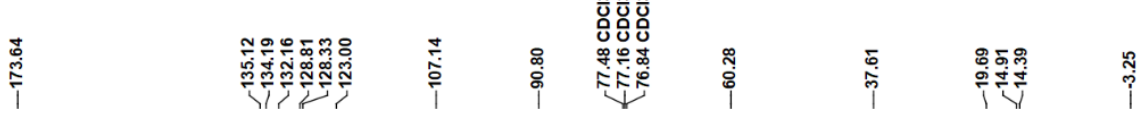

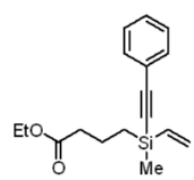

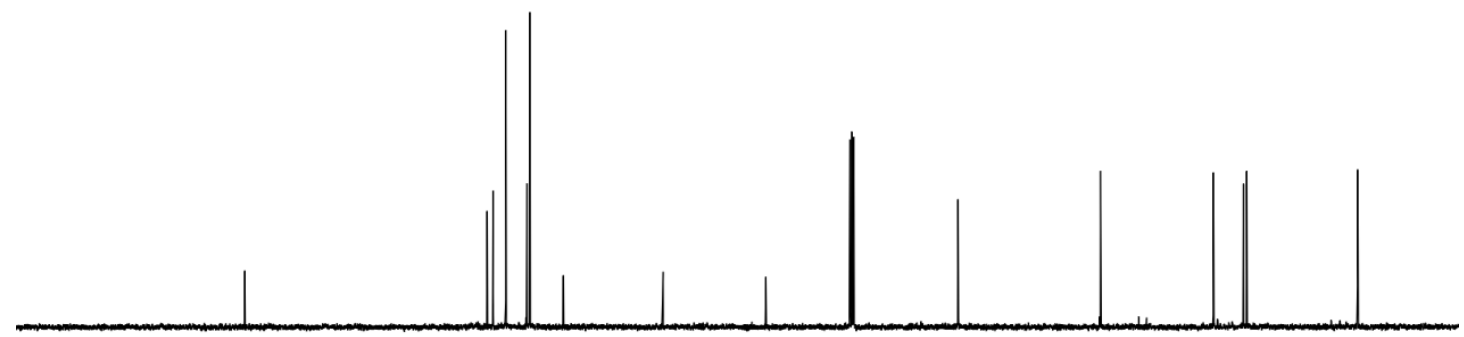

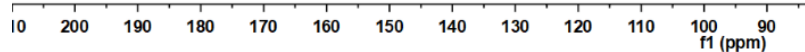


3ai; ${ }^{29} \mathrm{Si}$ NMR (79 MHz, $\mathrm{CDCl}_{3}$ )

๙<smiles>CC(C)(C)C(CCC(=O)O)C(C)(C)C1CCCC1</smiles>

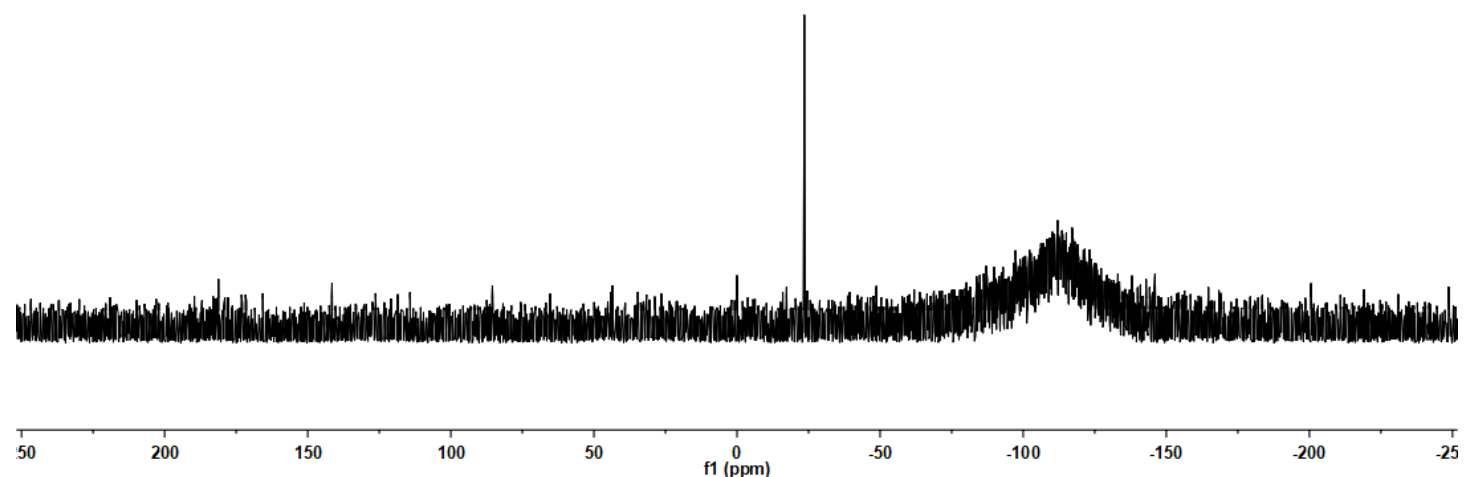


3aj; ${ }^{1} \mathrm{H}$ NMR (400 MHz, CDCl $)$; ${ }^{13} \mathrm{C}$ NMR (100 MHz, CDCl $)$
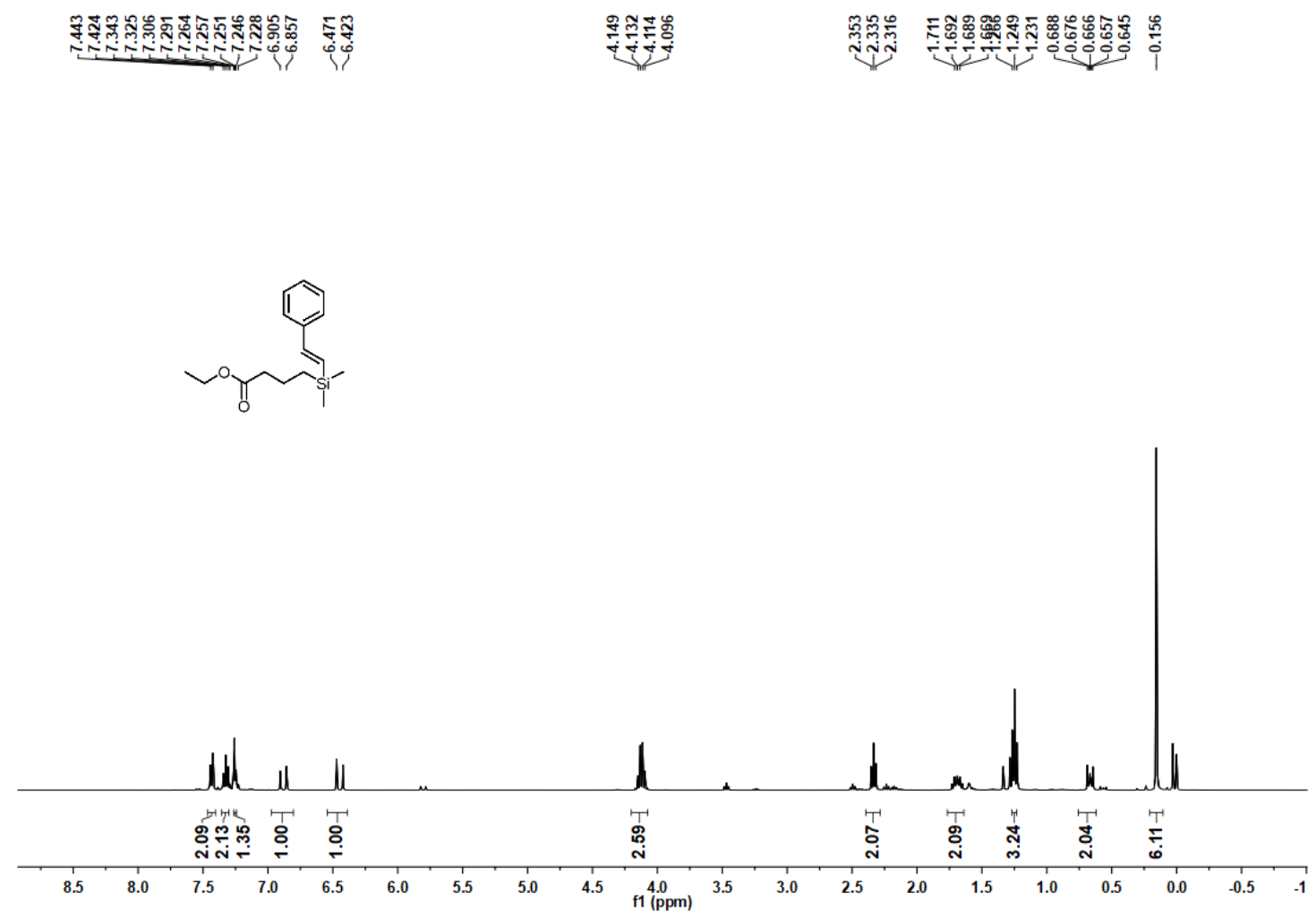

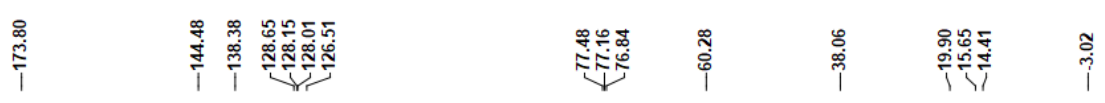
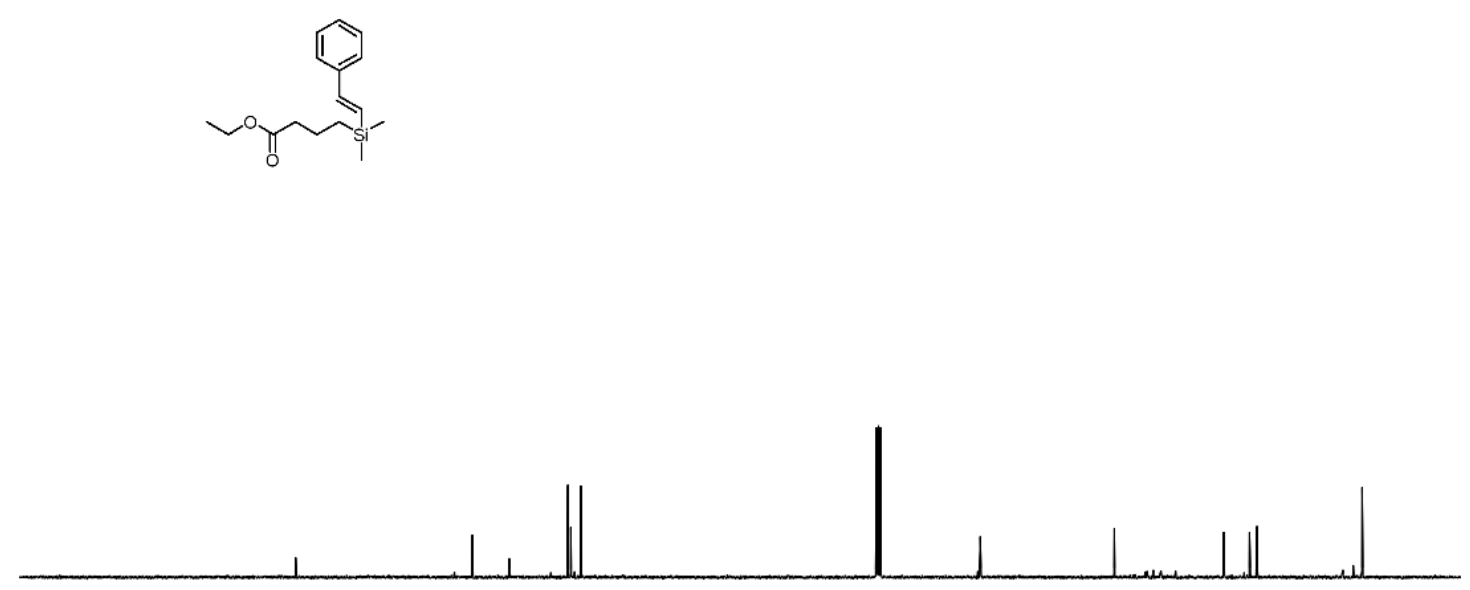

$\begin{array}{lllllllllll}210 & 200 & 190 & 180 & 170 & 160 & 150 & 140 & 130 & 120 & 110 \\ \mathrm{f} 1(\mathrm{ppm}) & 100 & 90\end{array}$ 
3aj; ${ }^{29} \mathrm{Si}$ NMR (79 MHz, $\left.\mathrm{CDCl}_{3}\right)$

i̊
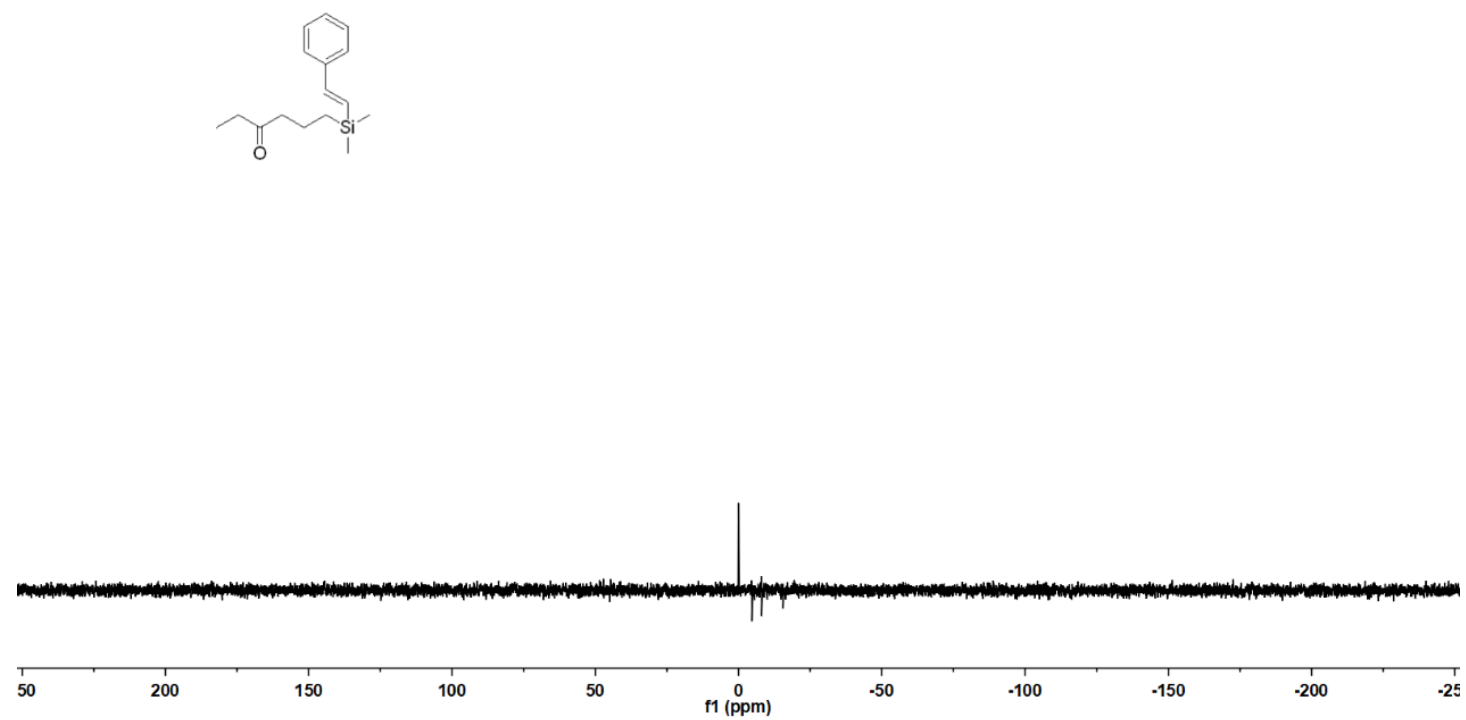
3ak; ${ }^{1} \mathrm{H}$ NMR (400 MHz, $\left.\mathrm{CDCl}_{3}\right) ;{ }^{13} \mathrm{C} \mathrm{NMR}\left(100 \mathrm{MHz}, \mathrm{CDCl}_{3}\right)$

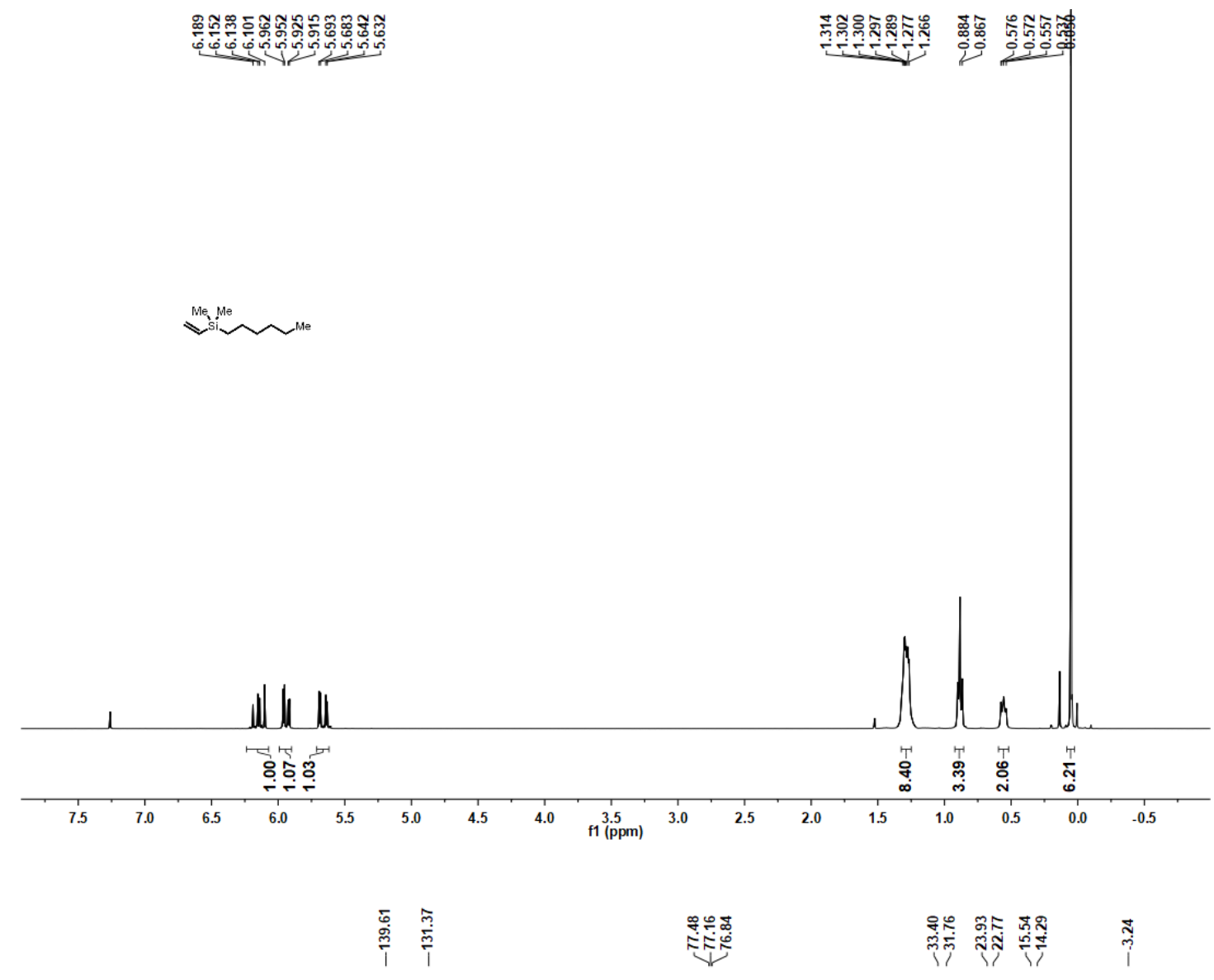

Me Me

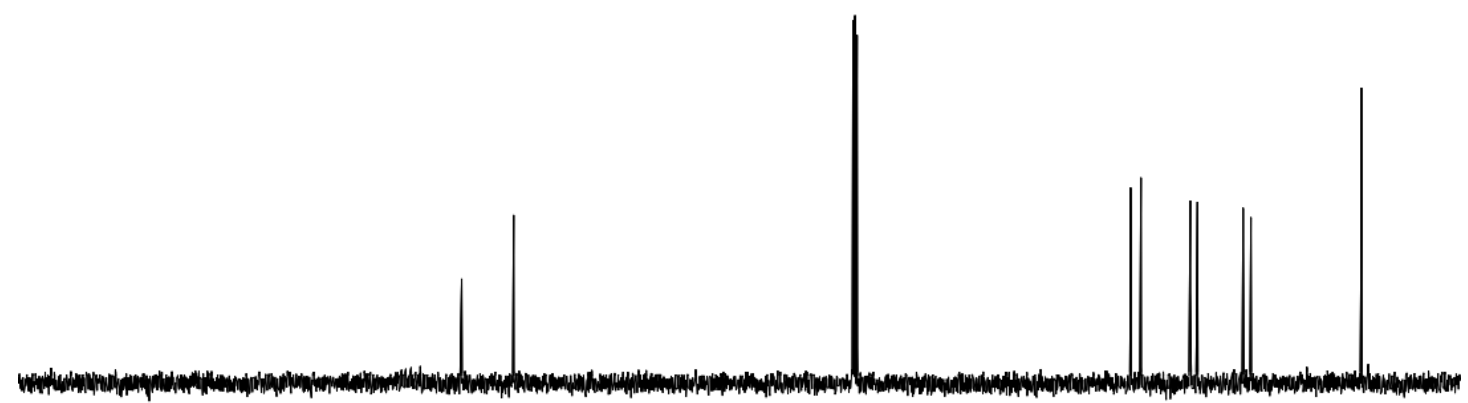

$\begin{array}{lllllllllllllllllllllll}1 & 10 & 200 & 190 & 180 & 170 & 160 & 150 & 140 & 130 & 120 & 110 & \underset{f}{100}(\mathrm{ppm}) & 80 & 70 & 60 & 50 & 40 & 30 & 20 & 10 & 0 & -10\end{array}$ 
3ak; ${ }^{29} \mathrm{Si}$ NMR (79 $\left.\mathrm{MHz}, \mathrm{CDCl}_{3}\right)$

ฟึ.

Me, Me

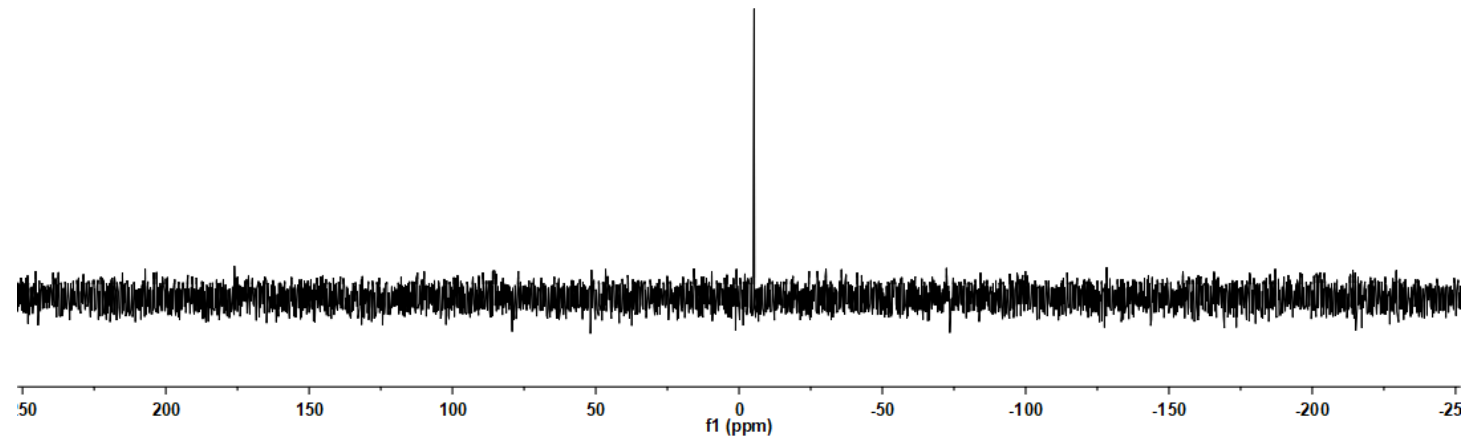


4; ${ }^{1} \mathrm{H}$ NMR (400 MHz, CDCl 3$) ;{ }^{13} \mathrm{C} \mathrm{NMR}\left(100 \mathrm{MHz}, \mathrm{CDCl}_{3}\right)$
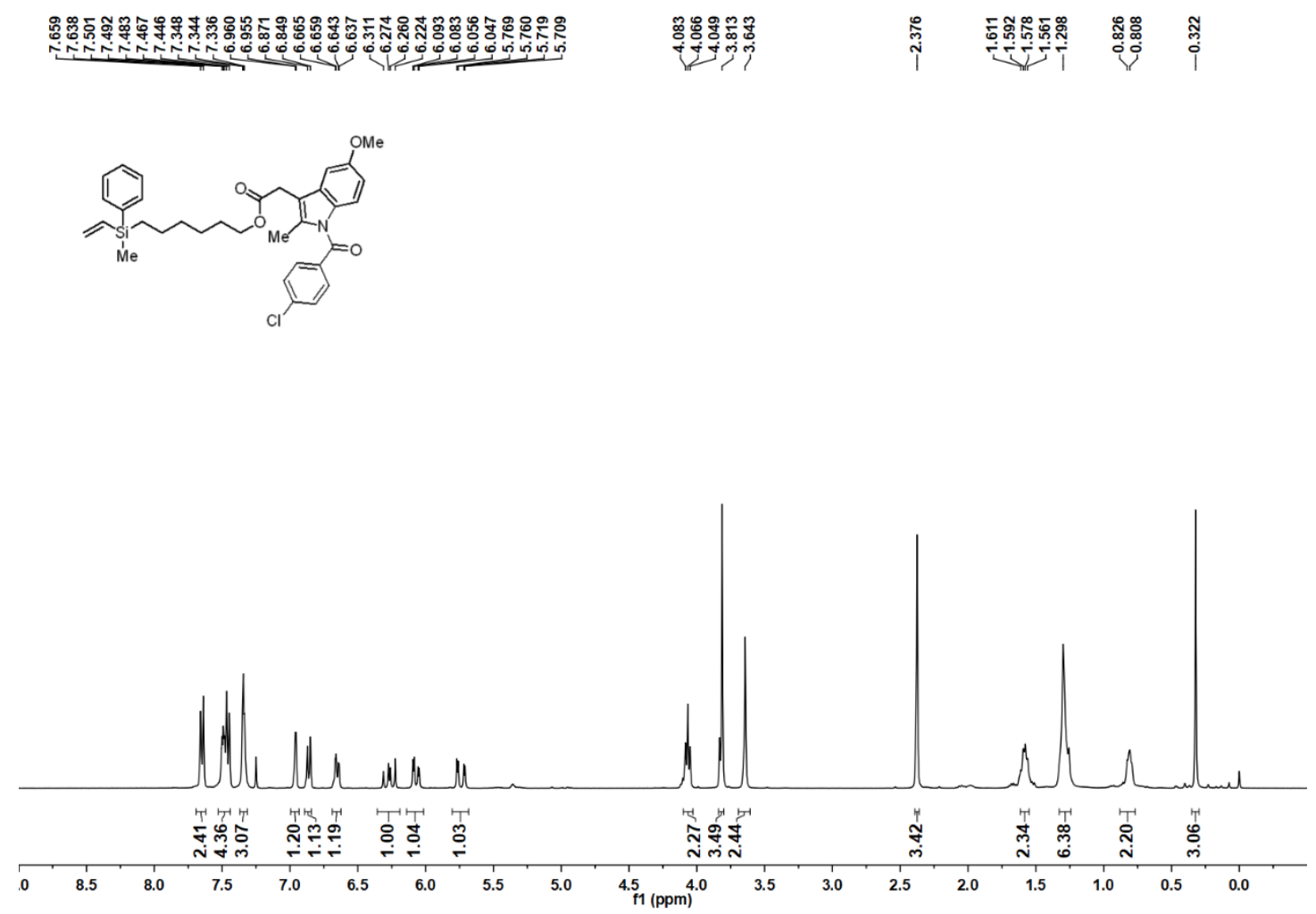

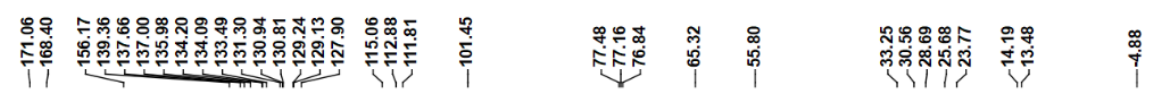
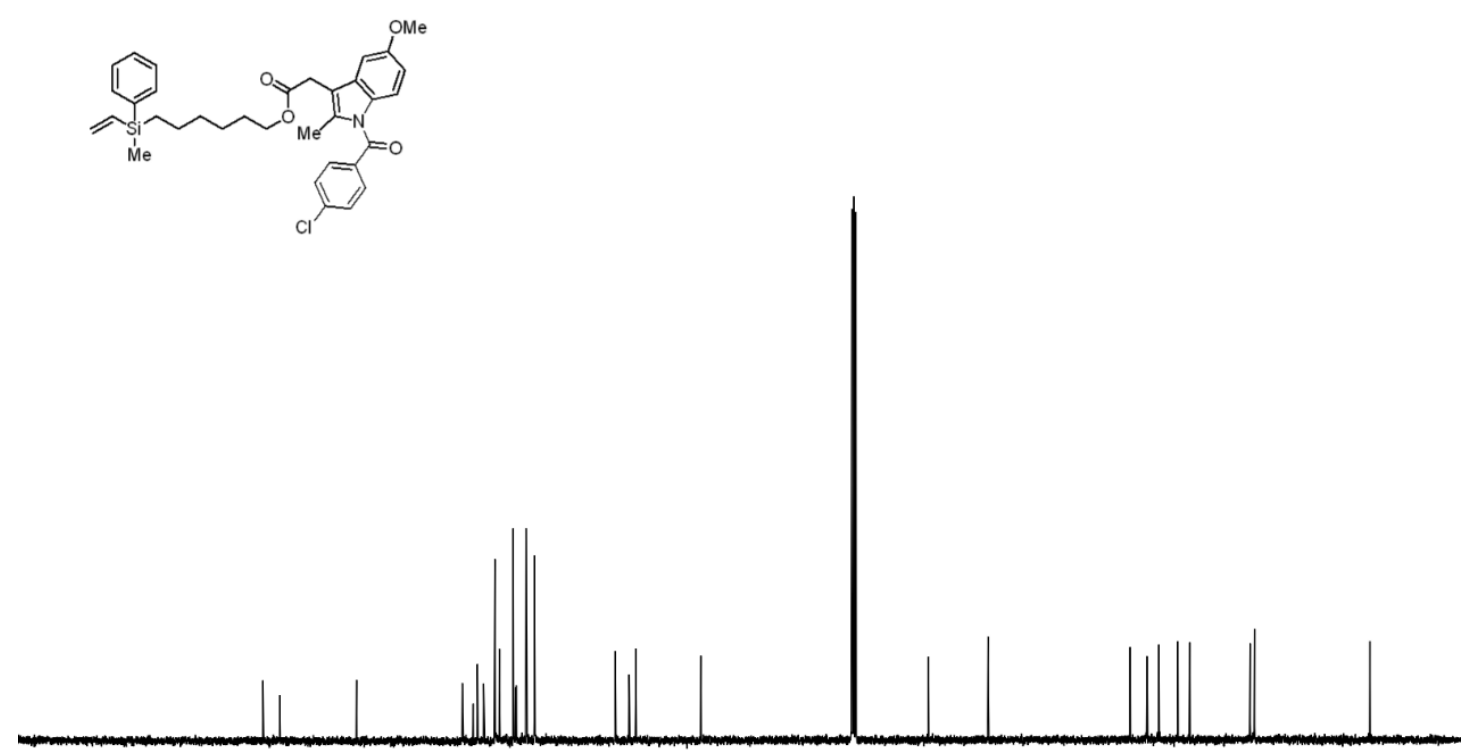

\begin{tabular}{lllllllllllllllllllllll}
\hline 10 & 200 & 190 & 180 & 170 & 160 & 150 & 140 & 130 & 120 & 110 & 100 & 90 & 80 & 70 & 60 & 50 & 40 & 30 & 20 & 10 & 0 & -10
\end{tabular} 
4; ${ }^{29} \mathrm{Si}$ NMR (79 MHz, $\left.\mathrm{CDCl}_{3}\right)$

†.

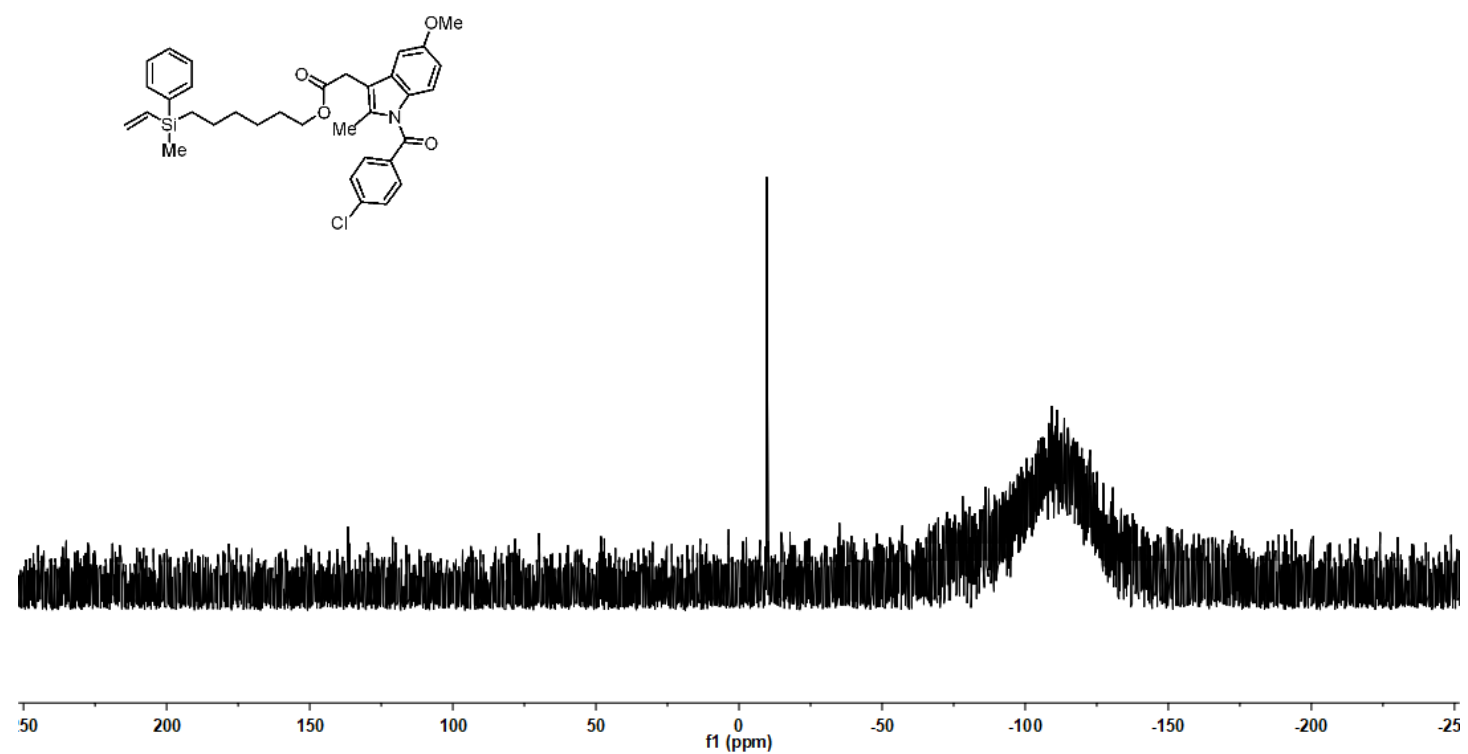


5; ${ }^{1} \mathrm{H}$ NMR (400 $\left.\mathrm{MHz}, \mathrm{CDCl}_{3}\right) ;{ }^{13} \mathrm{C}$ NMR (100 $\left.\mathrm{MHz}, \mathrm{CDCl}_{3}\right)$

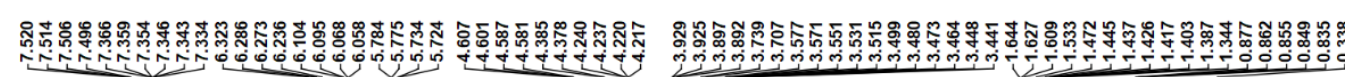

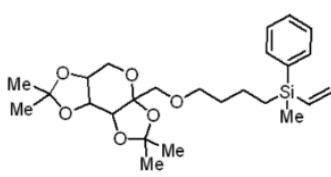

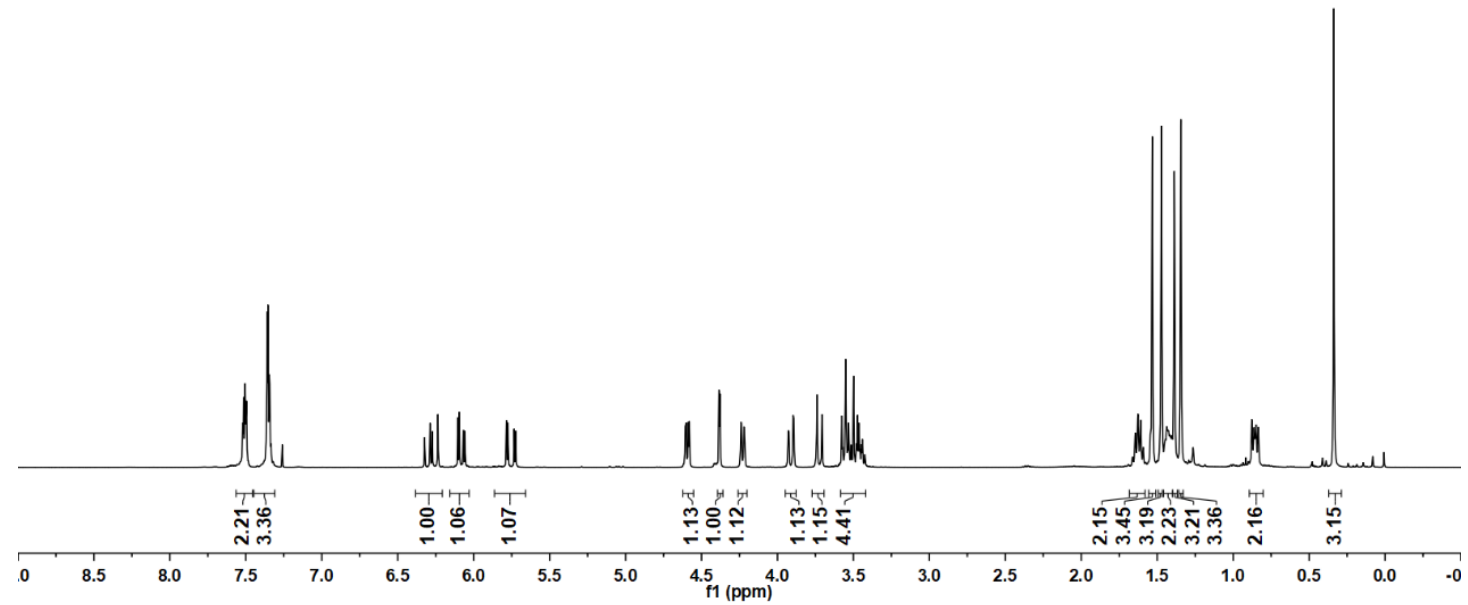

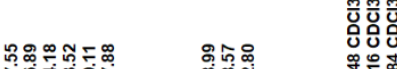

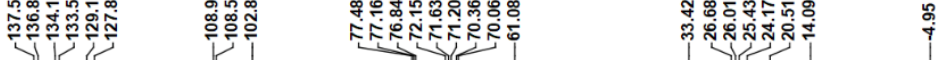<smiles>C=CN(CCCOCC12OCC3OC(C)(N)OC3C1OC2(C)C)c1ccccc1</smiles>

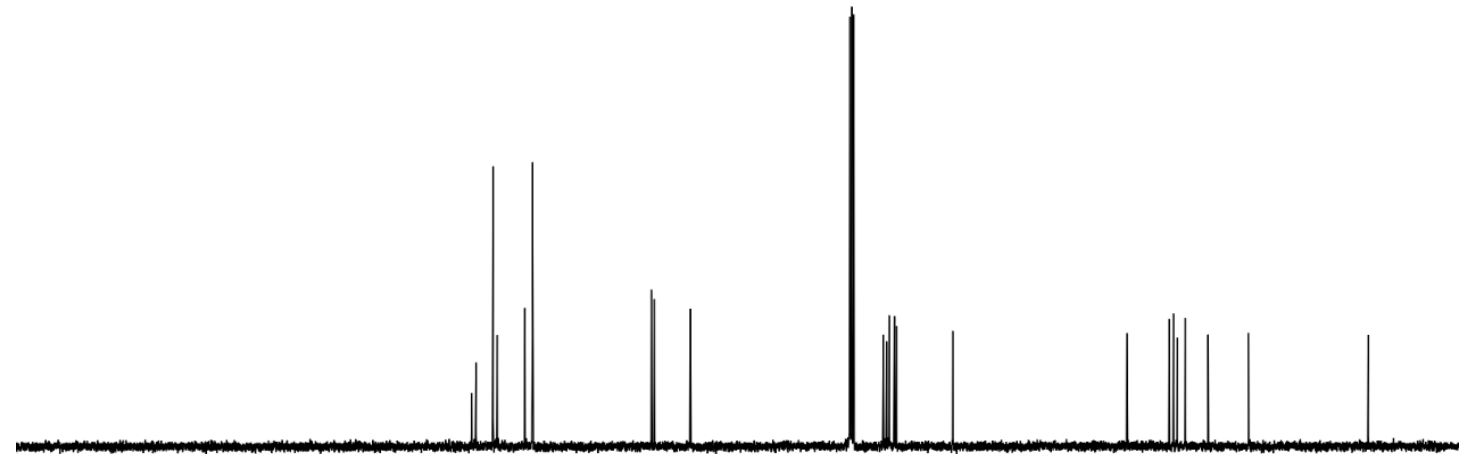

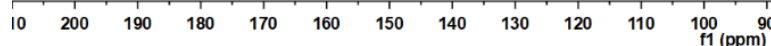


5; ${ }^{29} \mathrm{Si}$ NMR (79 MHz, $\left.\mathrm{CDCl}_{3}\right)$

Ð

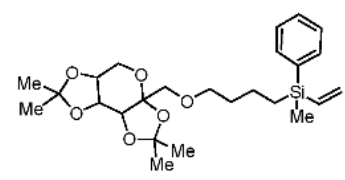

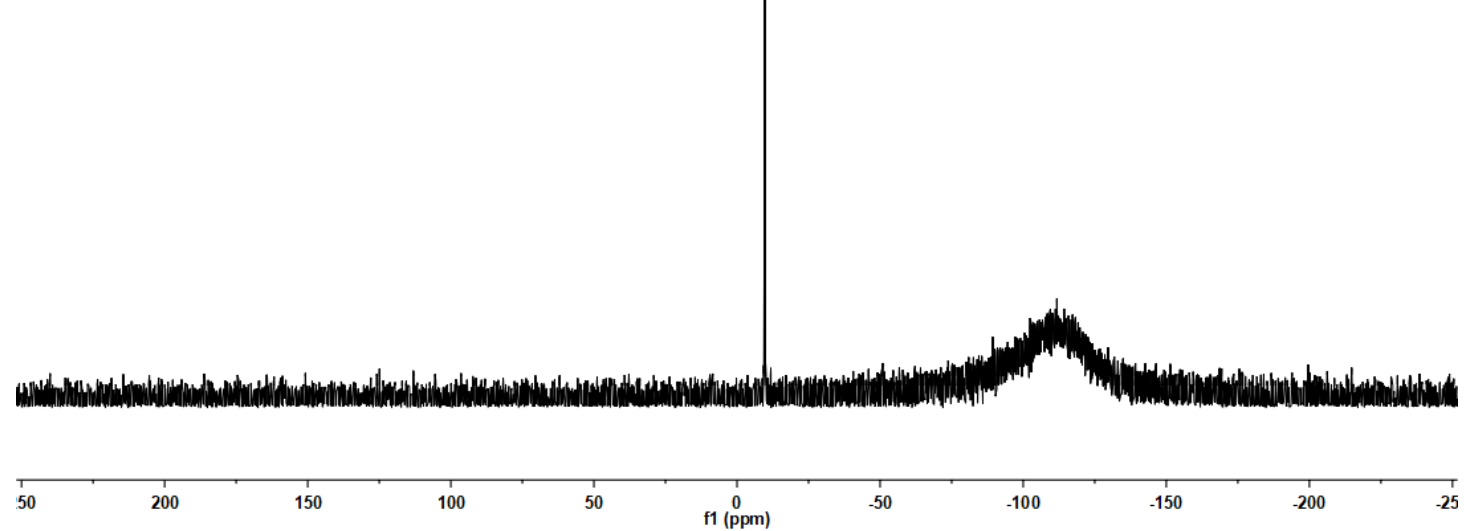


10; ${ }^{1} \mathrm{H}$ NMR (400 MHz, CDCl $) ;{ }^{13} \mathrm{C}$ NMR (100 MHz, CDCl 3$)$

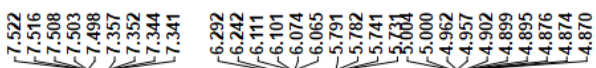

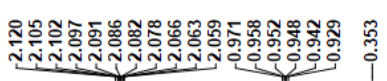

$\overbrace{\text { Si }}^{\text {Ph }}$
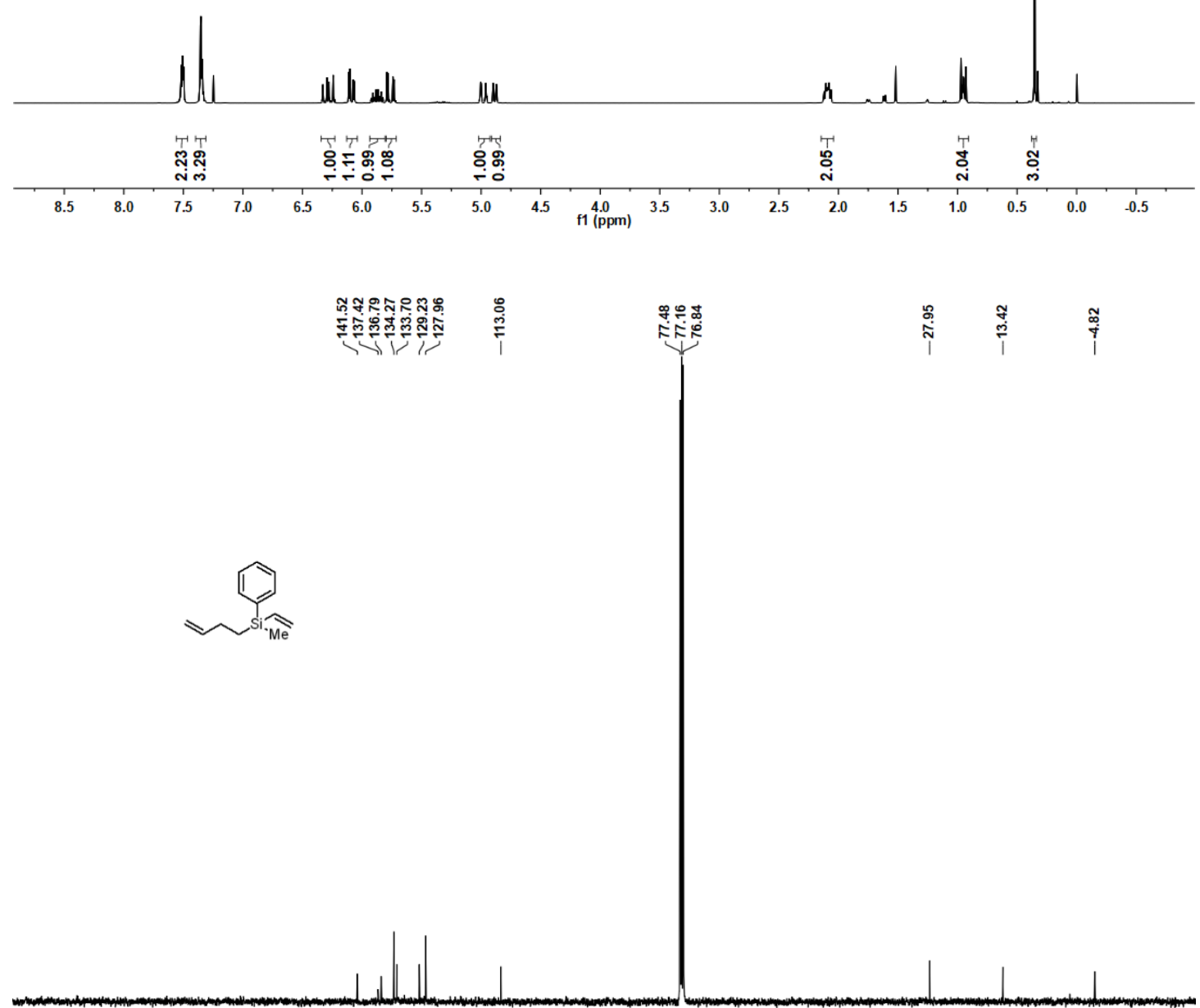

$\begin{array}{lllllllllllllllllllllllllllllllllll}10 & 200 & 190 & 180 & 170 & 160 & 150 & 140 & 130 & 120 & 110 & 100 & 90 & 80 & 70 & 60 & 50 & 40 & 30 & 20 & 10 & 0 & -10 & -20\end{array}$ 
10; ${ }^{29} \mathrm{Si}$ NMR (79 MHz, CDCl 3 )

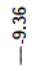

$\underbrace{\mathrm{Ph} \text { sí } \mathrm{Me}}$

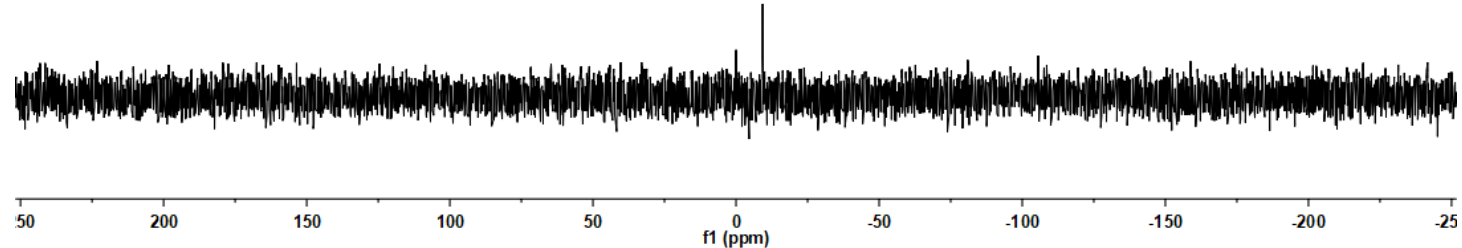

